\title{
ELECTROPHYSIOLOGICAL CHARACTERISTICS OF NEURONS IN THE DORSAL CORTEX OF THE INFERIOR COLLICULUS
}

\author{
A thesis submitted to \\ the Faculty of Graduate Studies and Research \\ in Partial Fulfillment of the requirements for the degree of \\ Doctor of Philosophy (Specialization in Neuroscience)
}

by

Hongyu Sun

Department of Psychology

Carleton University

March, 2008

C2008, Hongyu Sun 


$\begin{array}{ll}\begin{array}{l}\text { Library and } \\ \text { Archives Canada }\end{array} & \begin{array}{l}\text { Bibliothèque et } \\ \text { Archives Canada }\end{array} \\ \begin{array}{l}\text { Published Heritage } \\ \text { Branch }\end{array} & \begin{array}{l}\text { Direction du } \\ \text { Patrimoine de l'édition }\end{array} \\ \begin{array}{l}\text { 395 Wellington Street } \\ \text { Ottawa ON K1A 0N4 } \\ \text { Canada }\end{array} & \begin{array}{l}\text { 395, rue Wellington } \\ \text { Ottawa ON K1A 0N4 } \\ \text { Canada }\end{array}\end{array}$

Your file Votre référence ISBN: 978-0-494-40539-0

Ourfile Notre référence

ISBN: 978-0-494-40539-0

NOTICE:

The author has granted a nonexclusive license allowing Library and Archives Canada to reproduce, publish, archive, preserve, conserve, communicate to the public by telecommunication or on the Internet, loan, distribute and sell theses worldwide, for commercial or noncommercial purposes, in microform, paper, electronic and/or any other formats.

The author retains copyright ownership and moral rights in this thesis. Neither the thesis nor substantial extracts from it may be printed or otherwise reproduced without the author's permission.
AVIS:

L'auteur a accordé une licence non exclusive permettant à la Bibliothèque et Archives Canada de reproduire, publier, archiver, sauvegarder, conserver, transmettre au public par télécommunication ou par l'Internet, prêter, distribuer et vendre des thèses partout dans le monde, à des fins commerciales ou autres, sur support microforme, papier, électronique et/ou autres formats.

L'auteur conserve la propriété du droit d'auteur et des droits moraux qui protège cette thèse. $\mathrm{Ni}$ la thèse ni des extraits substantiels de celle-ci ne doivent être imprimés ou autrement reproduits sans son autorisation.
In compliance with the Canadian Privacy Act some supporting forms may have been removed from this thesis.

While these forms may be included in the document page count, their removal does not represent any loss of content from the thesis.
Conformément à la loi canadienne sur la protection de la vie privée, quelques formulaires secondaires ont été enlevés de cette thèse.

Bien que ces formulaires aient inclus dans la pagination, il n'y aura aucun contenu manquant.

\section{Canada}




\begin{abstract}
The inferior colliculus (IC) is considered to be an integration center in the auditory pathway. The dorsal cortex of the IC (ICD) is one of the major subdivisions of the IC, but how the ICD contributes to auditory processing is not clear. The purpose of this study was to investigate physiological characteristics of ICD neurons, including intrinsic membrane properties, synaptic response characteristics and physiological roles of $\mathrm{GABA}_{\mathrm{B}}$ receptors in membrane excitability and synaptic transmission of ICD neurons. Whole-cell patch clamp recordings were made from ICD neurons in rat brain slices.

In response to depolarizing current injection, the majority of neurons $(88.9 \%)$ showed a sustained-regular, a sustained-adapting or a buildup pattern. Following membrane hyperpolarization, a rebound depolarization and an anode break spike were observed in $51.7 \%$ of the sustained neurons, but not in the buildup neurons. In $78.4 \%$ of the ICD neurons, including non-rebound and rebound neurons, membrane excitability and firing behavior were altered in response to depolarizing current injection following hyperpolarization compared to those without pre-hyperpolarization. These results suggest that pre-hyperpolarization regulates membrane excitability not only when the neuron returns to the resting state, but also when the neuron is further depolarized. Generation of the rebound depolarization was primarily due to activation of low-threshold T-type $\mathrm{Ca}^{2+}$ channels. Some ICD neurons also displayed a $\mathrm{Ca}^{2+}$ mediated hump during depolarization and $\mathrm{Ca}^{2+}$-dependent $\mathrm{K}^{+}$current mediated after-hyperpolarization following an action potential.
\end{abstract}

ICD neurons responded to stimulation of the central nucleus of the IC or commissure of the IC (CoIC) with excitatory and inhibitory responses. The excitatory response was mediated by AMPA and NMDA receptors, whereas the inhibitory response was mediated by $\mathrm{GABA}_{\mathrm{A}}$ receptors. Repeated stimulation of the CoIC evoked long 
lasting inhibitory responses that were mediated by $\mathrm{GABA}_{\mathrm{B}}$ receptors. Activation of postsynaptic $\mathrm{GABA}_{\mathrm{B}}$ receptors by the specific $\mathrm{GABA}_{\mathrm{B}}$ receptor agonist, baclofen, profoundly suppressed cell's membrane excitability through affecting different ionic currents, i.e., inward rectifying $\mathrm{K}^{+}$current, $I_{\mathrm{h}}$, low-threshold $\mathrm{T}$ type $\mathrm{Ca}^{2+}$ current, highthreshold $\mathrm{Ca}^{2+}$ current, $\mathrm{Ca}^{2+}$-activated $\mathrm{K}^{+}$current and the sustained and transient A-type $\mathrm{K}^{+}$currents. Activation of presynaptic $\mathrm{GABA}_{\mathrm{B}}$ receptor by baclofen depressed both the AMPA and NMDA receptor mediated excitation, and the $\mathrm{GABA}_{\mathrm{A}}$ receptor mediated inhibition in ICD neurons.

The results of this study suggest that ICD neurons can provide various cellular mechanisms for integration and processing of auditory information. 


\section{Acknowledgements}

It is a great pleasure to thank the many people who have helped to make this thesis possible.

First of all I would like to express thanks to my supervisor, Dr. Shu Hui Wu. During the four and half years since I joined her research group in 2003, Dr. Wu's inspiration, guidance, and support made the electrophysiological study interesting for me. I was always deeply impressed by her enthusiasm and integral view on research. Dr. Wu is not only an excellent supervisor, but a good friend to me. I owe her countless gratitude for all her help during my doctoral study.

I wish to thank Dr. Jack Kelly who kept an eye on the progress of my research and was always available when I needed his advice. I would also like to thank my committee member Dr. Dan Mclntyre who spent time monitoring my research and reading and providing valuable comments on my $\mathrm{PhD}$ prospectus and thesis. I also want to offer my appreciation to the other members of my $\mathrm{PhD}$ examination committee, $\mathrm{Dr}$. John Lewis and Dr. Jeff Dawson, for the time they spent on this thesis. Thanks also, to Dr. Bruce Pappas for supervising my comprehensive exam.

I want to thank members of Dr. Wu lab, Drs. Chun-lei Ma and Tarun Ahuja, for teaching me how to make "blind" and "clean" whole cell patch clamp recordings. Also, I would like to thank Dr. Huiming Zhang for providing sound suggestions on my prospectus and generous help for my family.

I am also grateful to the secretaries, Etelle Bourassa and June Callender, in the Psychology Department and Diane Trenouth in the Institute of Neuroscience of Carleton University, for assisting me in many different ways.

Lastly, but most importantly, I wish to thank my wife, Hui Zhang, for her love, support and patience during my PhD study. Thanks to our sweet daughter, Gianna, who provided an additional and joyful dimension to our life during this period.

Hongyu Sun Ottawa, Canada 


\section{Table of Contents}

Abstract

Acknowledgements. i

Table of contents.

List of Abbreviations

List of Tables

List of Figures

Chapter 1. General introduction

1.1. Subdivision of the inferior colliculus

1.2. Central nucleus of the inferior colliculus (ICC) ........................ 3

1.2.1. Neuronal organization....................................... 3

1.2.2. Afferent and efferent projections of the ICC .................... 4

1.2.3. Functional role of the ICC................................... 6

1.3. External cortex of the inferior colliculus (ICX) ........................ 7

1.3.1. Neuronal organization...................................... 7

1.3.2. Afferent and efferent projections of the ICX..................... 8

1.3.3. Functional role of the ICX.................................... 9

1.4. Dorsal cortex of the inferior colliculus (ICD) ......................... 10

1.4.1. Neuronal organization.......................................... 10

1.4.2. Afferent projections of the ICD ............................. 12

1.4.3. Efferent projections of the ICD ................................ 14

1.4.4. Functional role of the ICD.................................... 15

1.5. Synaptic receptors in the IC.......................................... 16

1.5.1. Glutamate receptors............................................ 16

1.5.1.1. AMPA receptors...................................... 16

1.5.1.2. NMDA receptors.................................. 19

1.5.2. $\quad \gamma$-Aminobutyric acid (GABA) receptors........................ 21

1.5.2.1. GABA $_{\mathrm{A}}$ receptor..................................... 22

1.5.2.2. GABA $_{B}$ receptors.................................. 24

1.5.2.2.1. Molecular structure of $\mathrm{GABA}_{\mathrm{B}}$ receptor..... 24

1.5.2.2.2. Distribution of $\mathrm{GABA}_{B}$ receptor in the IC... 26

1.5.2.2.3. Presynaptic $\mathrm{GABA}_{B}$ receptor................ 27

1.5.2.2.4. Postsynaptic $\mathrm{GABA}_{\mathrm{B}}$ receptor................. 29

1.5.2.2.5. Functional role of the $\mathrm{GABA}_{B}$ receptors in the IC....................................... 30

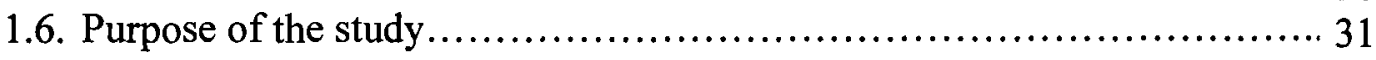

1.6.1. Intrinsic membrane properties of ICD neurons.................. 32 
1.6.2. Contribution of $\mathrm{GABA}_{B}$ receptors to membrane excitability and synaptic transmissionof ICD neurons

Chapter 2. Intrinsic membrane properties of neurons in the rat's ICD: effects of prehyperpolarization on membrane excitability.......................... 36

2.1. Introduction......................................................... 36

2.2. Materials and Methods.......................................... 37

2.2.1. Preparation of brain slices................................. 37

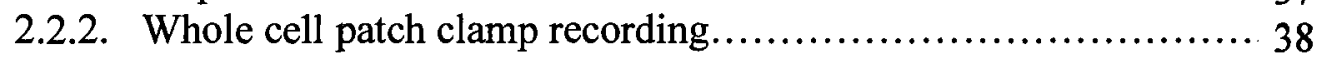

2.2.3. Data analysis.............................................. 39

2.3. Results....................................................... 40

2.3.1. Responses to injection of depolarizing currents.................. 41

2.3.2. Responses to injection of hyperpolarizing currents............... 44

2.3.3. Responses to depolarizing currents following membrane hyperpolarization........................................ 45

2.4. Discussion..................................................... 74

2.4.1. Intrinsic membrane properties of ICD neurons around hearing onset.................................................. 74

2.4.2. Modification of membrane excitability by prehyperpoalrization in NR neurons.............................................. 75

2.4.3. Modification of membrane excitability by prehyperpoalrization in rebound neurons........................................... 78

2.4.4. Functional implications of different modification ............... 81

Chapter 3. Contribution of calcium to membrane excitability of neurons in the rat's dorsal cortex of the inferior colliculus.............................. 85

3.1. Introduction................................................... 85

3.2. Materials and Methods............................................ 86

3.2.1. Preparation of brain slices................................. 86

3.2.2. Solution and drugs.......................................... 86

3.2.3. Whole cell patch clamp recording............................ 86

3.2.4. Data analysis............................................. 87

3.3. Results...................................................... 89

3.3.1. Afterhyperpolarization mediated by calcium conductance......... 89

3.3.2. $\mathrm{Ca}^{2+}$-activated $\mathrm{K}^{+}$current mediated sAHP ...................... 90

3.3.3. Calcium-mediated depolarization and spikes ................... 90

3.3.4. Calcium mediated rebound depolarization following hyperpolarization........................................... 92

3.3.5. Generation of the rebound and anode break APs depended on magnitude of preceding hyperpolarization ..................... 94

3.3.6. Generation of the rebound and anode break APs depended on duration of preceding hyperpolarization. 
3.3.7. Level and duration of hyperpolarization required for generation of minimum and maximum rebound

3.4. Discussion

3.4.1. The role of $\mathrm{Ca}^{2+}$ in spiking....

3.4.2. Contribution of $\mathrm{Ca}^{2+}$ mediated rebound to membrane excitability

3.4.3. Functional implications

Chapter 4. Physiological roles of $\mathrm{GABA}_{\mathrm{B}}$ receptors in neurons of the dorsal cortex of the inferior colliculus.

4.1. Introduction.

4.2. Materials and Methods.

4.2.1. Preparation of brain slices

4.2.2. Whole cell patch clamp recording............................ 132

4.2.3. Synaptic stimulation....................................... 133

4.2.4. Solutions and drugs...................................... 134

4.2.5. Data analysis............................................ 135

4.3. Results....................................................... 136

4.3.1. Effects of baclofen on passive membrane properties of ICD neurons.

4.3.2. Effects of baclofen on active membrane properties of ICD neurons.

4.3.2.1. Effects of $\mathrm{GABA}_{\mathrm{B}}$ receptor activation on firing behaviour of ICD neurons

4.3.2.2. Effects of $\mathrm{GABA}_{\mathrm{B}}$ receptor activation on $\mathrm{AP}$ properties of ICD neurons.

4.3.3. Baclofen evokes an inward rectifying $\mathrm{K}^{+}$current ............... 141

4.3.4. CGP35348 significantly reduced baclofen mediated inhibition.... 142

4.3.5. Modulation of Ih by $\mathrm{GABA}_{\mathrm{B}}$ receptor activation ................ 143

4.3.6. Modulation of rebound depolarization by $\mathrm{GABA}_{\mathrm{B}}$ receptor activation

4.3.7. Modulation of depolarization hump by activation of $\mathrm{GABA}_{B}$ receptor.

4.3.8. Inhibition of $\mathrm{A}$-current by activation of $\mathrm{GABA}_{\mathrm{B}}$ receptors........ 148

4.3.9. Stimulus-evoked postsynaptic $\mathrm{GABA}_{\mathrm{B}}$ receptor-mediated response

4.3.10. Presynaptic $\mathrm{GABA}_{\mathrm{B}}$ receptors modulate neuronal transmission in ICD.

4.3.10.1. Synaptic responses mediated by AMPA, NMDA and $\mathrm{GABA}_{\mathrm{A}}$ receptors in ICD neurons

4.3.10.2. Presynaptic $\mathrm{GABA}_{B}$ receptor activation inhibits synaptic transmission in ICD.

4.4. Discussion 
4.4.1. Postsynaptic $\mathrm{GABA}_{B}$ receptors in the ICD neurons

4.4.2. $\mathrm{GABA}_{\mathrm{B}}$ receptors suppress the membrane excitability of ICD neurons....................................................... 186

4.4.2.1. Modulation of voltage-dependent $\mathrm{K}^{+}$conductance........ 188

4.4.2.2. Modulation of $\mathrm{Ca}^{2+}$ currents .......................... 189

4.4.2.3. Modulation of $\mathrm{Ca}^{2+}$-activated potassium channels...... 191

4.4.3. $\mathrm{GABA}_{\mathrm{B}}$ receptors modulate $I_{h}$ currents in ICD neurons ........... 192

4.4.4. $\mathrm{GABA}_{\mathrm{B}}$ receptors modulate T-type calcium channel-mediated rebound depolarization in ICD neurons ....................... 193

4.4.5. Synaptic transmission between ICC and ICD.................... 195

4.4.6. Presynaptic $\mathrm{GABA}_{\mathrm{B}}$ receptor modulates synaptic transmission in the ICD ................................................ 196

4.4.7. Functional significance of $\mathrm{GABA}_{\mathrm{B}}$ receptors in ICD neurons ….... 197

Chapter 5. Summary ..................................................... 199

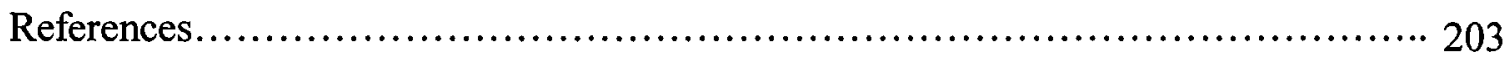




\section{List of Abbreviations}

4-AP

$\mathrm{AC}$

ACSF

AHP

AMPA

AP

APV

AR

AVCN

BIC

cAHP

$\mathrm{CN}$

CNQX

CNS

CoIC

CPP

DCN

DNLL

EPSC

EPSP

$\mathrm{E}_{R E V}$

fAHP

FM

FSL

GABA

HEPES

HRP

IC

ICC

ICD

ICX

iGluRs

$\mathrm{I}_{h}$

IID 4-aminopyridine

auditory cortex

artificial cerebrospinal fluid

after-hyperpolarization

$\alpha$-amino-3-hydroxy-5-methyl-4-isoxazolepropionic acid action potential

$( \pm)$-2-amino-5-phosphonovaleric acid adaptation ratio

anteroventral cochlear nuclei

brachium of the inferior colliculus

combined afterhyperpolarization

cochlear nucleus

6-cyano-7-nitroquinoxaline-2, 3-dione

central nervous system

commissure of the inferior colliculus

3-(2-carboxypiperazin-4-yl)-propyl-1-phosphonic acid dorsal cochlear nucleus

dorsal nucleus of the lateral lemniscus

excitatory postsynaptic current

excitatory postsynaptic potential

reversal potential

fast afterhyperpolarization

frequency-modulated

first spike latency

$\gamma$-aminobutyric acid

$N$-2-hydroxyethylpiperazine- $N$ '-2-ethanesulfonic acid

Horseradish peroxidase

inferior colliculus

central nucleus of the inferior colliculus

dorsal cortex of the inferior colliculus

external cortex of the inferior Colliculus

ionotropic glutamate receptors

hyperpolarization-activated inward current

interaural intensity difference 
$I K_{A}$

$I K_{s}$

$I K_{T}$

iMP

IPD

IPSC

IPSP

ITD

I-V

LIV-BP

LL

LNTB

LSO

LTP

MGB

mGluRs

MNTB

MSO

NADPH

NMDA

NR

PHA-L

PVCN

RD

$\mathrm{R}_{\text {input }}$

sAHP

$\mathrm{SC}$

SOC

SPN

$\tau$

TEA

TTX

VCN

VNLL

VNTB transient $\mathrm{A}$ type potassium current

sustained potassium current

total voltage-dependent potassium current

ipsilateral medial plexus

interaural phase difference

inhibitory postsynaptic current

inhibitory postsynaptic potential

interaural time difference

current-voltage

leucine isoleucine valine binding protein

lateral lemniscus

lateral nucleus of the trapezoid body

lateral superior olive

long-term potentiation

medial geniculate body

metabotropic glutamate receptors

medial nucleus of the trapezoid body

medial superior oliver

nicotinamide adenine dinucleotide phosphate

$N$-methyl-D-aspartate

non-rebound

phaseolus vulgaris-leucoagglutinin

posteroventral cochlear nuclei

rebound depolarization

input resistance

slow afterhyperpolarization

superior colliculus

superior olivary complex

superior paraolivary nucleus

time constant

tetraethylammonium chloride

tetrodotoxin

ventral cochlear nucleus

ventral nucleus of the lateral lemniscus

ventromedial nucleus of the trapezoid body 


\section{List of Tables}

Table

Page

2.1 Intrinsic membrane characteristics of ICD neurons........................ 52

2.2 Comparison of membrane characteristics of regular and adapting ICD neurons in three age group........................................... 53

3.1 Comparison of membrane characteristics of ICD neurons with rebound firing in three age groups.............................................. 101 


\section{List of Figures}

Figure

Page

1.1 Schematic diagram of the coronal section through the inferior colliculus...... 35

2.1 Three firing patterns classified according to responses to depolarizing current injection................................................... 54

2.2 Responses to hyperpolarizing current injection in two neurons.............. 56

2.3 Pre-hyperpolarization did not affect the firing pattern in a sustained-regular neuron.

2.4 A sustained firing pattern was changed to a pauser pattern by prehyperpolarization in a neuron.

2.5 A firing pattern was changed from sustained to buildup or remained buildup after pre-hyperpolarization in two neurons.

2.6 A regular firing pattern was changed to adapting and firing rates were increased by pre-hyperpolarization in a rebound neuron.

2.7 A firing pattern was changed from sustained to pauser or onset, and firing rates were decreased by pre-hyperpolarization in two rebound neurons.

2.8 A firing pattern was changed from sustained to irregular by pre-hyperpolarization in a rebound neuron.

2.9 Comparisons of the first spike latency and firing rate between responses to depolarizing current injection with and without pre-hyperpolarization.

3.1 Characteristics of three types of AHP................................. 102

3.2 The AHP was mediated by $\mathrm{Ca}^{2+}$ activated $\mathrm{K}^{+}$current......................... 104

$3.3 \mathrm{Ca}^{2+}$ mediated hump and spikes......................................... 106

3.4 The rebound was mediated by $\mathrm{Ca}^{2+}$ and comparisons of anode break spike in rebound and non-rebound neurons.

3.5 The latency and threshold of anode break spikes were related to the level of hyperpolarizing current and the slope of the rebound...................... 110

3.6 The relationships of the amplitude, area and kinetics of the rebound to the level of negative current injection....................................... 112

3.7 The relationship between the latency of anode break spikes and the duration of current injection.

3.8 The relationships of the amplitude, area and kinetics of the rebound to the duration of negative current injection.

3.9 The level and duration of hyperpolarizing current required to induce minimum or maximum rebound. 
4.1 Baclofen hyperpolarized the cell membrane and decreased the input resistance in ICD neurons.............................................. 154

4.2 Baclofen affected the resting potential and input resistance of ICD neurons in a concentration-dependent manner................................... 156

4.3 Baclofen depressed the membrane excitability of ICD neurons............... 158

4.4 Baclofen modulated action potential properties in ICD neurons............... 160

4.5 Baclofen evoked an inward rectifying $\mathrm{K}^{+}$current in ICD neurons.............. 162

4.6 Baclofen-induced current was blocked by barium............................ 164

4.7 CGP35348 blocked the effects of baclofen in ICD neurons.................... 166

4.8 Baclofen suppressed Ih currents in ICD neurons........................... 168

4.9 Baclofen suppressed T-type $\mathrm{Ca}^{2+}$ mediated rebound depolarization in ICD

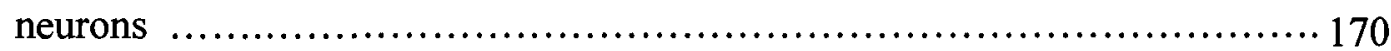

4.10 Baclofen attenuated the high-threshold $\mathrm{Ca}^{2+}$ currents in ICD neurons........... 172

4.11 Baclofen prolonged the delay in firing in ICD neurons...................... 174

4.12 Baclofen enhances A-type and sustained voltage-dependent $\mathrm{K}^{+}$currents in

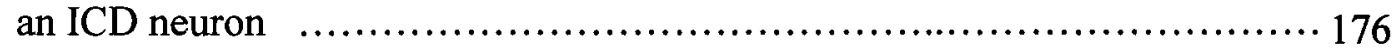

4.13 GABAB receptor mediated IPSCs in ICD neurons......................... 178

4.14 Baclofen modulated excitatory synaptic transmission from ICC to ICD........ 180

4.15 Baclofen suppressed IPSCs mediated by $\mathrm{GABA}_{\mathrm{A}}$ receptors in an ICD neuron. 182 


\section{Chapter 1}

\section{General introduction: Background to the research}

One of the major goals in auditory research is to understand how we hear, i.e., how acoustic information is progressively transformed along the auditory pathway (Pollak et al., 2003). To achieve this goal has been very difficult because of the complexity of the auditory system. The auditory system is composed of a number of nuclei that are connected by parallel and series pathways. The pathways from various neuronal groups in the auditory lower brainstem ultimately converge on a common destination, the inferior colliculus (IC). Besides the ascending inputs from nearly all auditory nuclei in the lower brainstem, the $\mathrm{IC}$ also receives the inputs from the opposite IC and the descending projections from auditory cortex. In addition, the IC is also reported to receive projections from ascending somatosensory pathways, indicating that auditory information is integrated with other somatosensory information. The IC provides the principal source of innervations to the medial geniculate body and indirectly to the auditory cortex. Therefore, the IC is the nexus of the auditory system because it integrates almost all ascending acoustic information, and then determines the form of the information output to higher auditory centers. The IC has been the subject of numerous studies for more than 30 years. In vivo, several studies have acquired significant results on the physiological properties of IC neurons using extracellular recording techniques. Recent in vitro studies also provide important information about the physiology of IC neurons. However, the exact nature and functions of the IC neurons remain an enigma.

\subsection{Subdivisions of the IC}

The IC is visible on the dorsal surface of the midbrain and sits immediately below 
the superior colliculus. It is among the largest auditory nuclei in the vertebrate brain. In cat, the approximate size of the IC is $5.5 \mathrm{~mm}$ in the dorsoventral, $5 \mathrm{~mm}$ in the mediolateral and $3 \mathrm{~mm}$ in the rostrocaudal extension (Ehret, 1997). Although the IC is easy to be distinguished from other midbrain structures, understanding the functional organization of the IC has been a problem (Oliver, 2005). The internal structure of the IC is characterized by the distribution of different cell types, patterns of dendritic branching and the termination of afferent, intrinsic and commissural fibers (Casseday et al., 2002). Several attempts have been performed to subdivide the IC using different methods such as Golgi staining, Nissl staining, and immunocytochemistry with the hope that these subdivisions would correspond to functional zones. Ramon Cajal (1909) provided early interpretations of general cytoarchitecture of the mammal IC using Golgi-impregnated materials and identified three major subdivisions: the nucleus, the internuclear cortex and the lateral cortex. Using Nissl-stained sections, Golgi impregnations and myelin stains, Faye-Lund and Osen (1985) subdivided the rat IC into three main areas: the central nucleus (ICC), the laterally and rostrally placed external cortex (ICX), and the dorsal cortex (ICD). Morest and Oliver (1984) presented further subdivisions based on their unique neuropil and neuronal populations in IC with the Golgi-impregnated materials from cat. They subdivided the IC into four subdivisions: the medial, central, lateral and ventral part. Across species, the IC shows striking homogeneities in cytoarchitecture and connectivity, such as cat (Morest, 1966; Rockel and Jones, 1973; Morest and Oliver, 1984), rat (Faye-Lund, 1985; Faye-Lund and Osen, 1985; Coleman and Clerici, 1987), opossum (Willard and Martin, 1983), monkey (Fitzpatrick, 1975), and even human (Geniec and Morest, 1971). However, there are still some minor species variations. The 
size of the subdivisions may be a little bit different in different species and some subdivision may be absent or hypertrophic (Morest and Oliver, 1984; Zook et al., 1985; Malmierca, 2003). In summary, the IC can be viewed as a slightly caudal, ventrolateral ICC encapsulated by the ICD dorsally and by the ICX ventrally, laterally and rostrally (Fig. 1.1). Every subdivision has unique afferent and efferent connections. The cell type and size, dendritic field shape and organization are also different in these three nuclei. The neurons differ in their response to acoustic stimuli (Huffman and Henson, 1990).

\subsection{Central nucleus of the inferior colliculus (ICC)}

\subsubsection{Neuronal organization}

The central nucleus is the main division of the mammalian IC. It is characterized by the presence of fibrodendritic laminae distinguishable in Golgi materials, which is composed of disc-shaped neurons and the laminar plexus of afferent fibers (Oliver, 2005). This laminar organization offers the structural basis for the tonotopic organization and has been observed in almost every species studied (Morest and Oliver, 1984; FayeLund and Osen, 1985; Zook et al., 1985; Kelly et al., 1991; Malmierca et al., 1993, 1995; Pierson and Snyder-keller, 1994; Malmierca, 2003). Two major cell types have been defined by morphology in the ICC of the cat: disc-shaped neurons and stellate cells (Oliver, 2005). Disc-shaped neurons are the most common cell type in the ICC. They have dendritic fields parallel to one another and contribute a distinct laminae appearance in Golgi stains. The thickness of the dendritic trees determines the width of the laminae which is about $70-150 \mu \mathrm{m}$. Stellate neurons, the second main cell type, represent $<25 \%$ of the total population. Their dendrites extend beyond the single fibrodendritic laminae into adjacent laminae. In the rat, Malmierca et al., (1993) described two types of neurons: flat 
cells and less flat cells. The high frequency part of the rat ICC has layers where flat cells alternate with less-flat cells. One flat cell and one less-flat cell are supposed to form the laminae unit. A laminae thickness is about $150-170 \mu \mathrm{m}$. In lower frequency part of the rat ICC, alternating cell types have not been found (Malmierca et al., 1993, Oliver, 2005).

\subsubsection{Afferent and efferent projections of the ICC}

Using degeneration techniques, radioactive tracer and horseradish peroxidase or fluorescent dye labelling methods, several studies have found the evidence of ascending input to the ICC (Ehret, 1997). The ICC receives ascending inputs from neurons in the lower auditory brain stem. In the rat, the ICC receives inputs from neurons of the cochlear complex consisting of the dorsal (DCN), anteroventral (AVCN) and posteroventral cochlear nuclei (PVCN) (Faye-Lund, 1986; Coleman and Clerici, 1987; Cant, 2005). The inputs are also from the superior olivary complex (SOC) including medial superior olive (MSO), lateral superior olive (LSO), superior paraolivary nucleus (SPN), and medial, lateral and ventromedial nuclei of the trapezoid body (MNTB, LNTB, VNTB) (Schofield, 2005). Moreover, the great majority of auditory ascending fibers from the lateral lemniscal nuclei terminates in the ICC and represents the largest single source of the inputs (Brunso-Bechtold et al., 1981; Shneiderman et al., 1988; Ehret, 1997; Oliver, 2005). The source of the projections mentioned above can be monaural (i.e., DCN, VCN, VNLL) or binaural (i.e., MSO, LSO, DNLL) (Kelly and Zhang, 2002). They send projections from either contralateral side (i.e., $\mathrm{DCN}, \mathrm{VCN}$ ), ipsilateral side (i.e., SPN, VNLL), or bilateral sides (i.e., MSO, LSO, DNLL) (Kelly and Zhang, 2002). The descending projections originating from auditory cortex to the ICC have been summarized by Huffman and Henson (1990). The ICC receives only limited descending 
projections. But it may have some indirect influence from the ICD, ICX or the superior colliculus (SC), which has been demonstrated in rat (Coleman et al., 1984; Faye-Lund, 1985), monkey (Kuypers and Lawrence, 1967) and cat (Andersen et al., 1980; Morest and Oliver, 1984).

The efferent (ascending) projections from the ICC originate from both the flat and less-flat neurons (Peruzzi et al., 1997). Ipsilaterally, the ICC sends the major ascending projections to the ipsilateral medial and ventral divisions of the medial geniculate body (MGB) in a strictly tonotopic manner, but also with some minor inputs to the contralateral MGB (Cat: Oliver, 1984; Guinea pig: Malmierca et al., 1996; Tree shrew: Oliver and Hall, 1978). The ICC also projects some minor fibers to the deep layers of the ipsilateral superior colliculus (SC) (Covey et al., 1987; Frisina et al., 1997). In addition, the ICC sends the fibers, named intrinsic connection, to the other subdivisions, i.e., ICD and ICX, of the IC on the same side (Faye-Lund and Osen, 1985; Saldaña and Merchan, 1992). A study by Saldaña and Merchan (1992) investigated the organization of the intrinsic connections of the rat. They injected an anterograde tracer, phaseolus vulgarisleucoagglutinin (PHA-L), into the ICC. The results showed that the intrinsic fibers formed "sheets" which were parallel to the isofrequency contours of the ICC and extended into the ICD and ICX. More recent study by Malmierca et al, (2003) reported the similar results. The majority of these intrinsic projections are thought to be excitatory (Fremouw et al., 1999; Yang et al., 2000). Moreover, the ICC also sends the projections to the contralateral ICC with some minor inputs to the contralateral ICD and ICX (Moore and Goldberg, 1963, 1966; Druga and Syka, 1984; Saldana and Merchan, 1992; Frisina et al., 1997). The fibers that interconnect the two sides are referred as commissural fibers. 
These commissural fibers may be either glutamatergic or GABAergic (Saint-Marie, 1996; Yang et al., 2000) and have either an excitatory or inhibitory effect on the contralateral ICC (Smith, 1992; Li et al., 1998, 1999; Moore et al, 1998). They may affect frequency response area, temporal properties and binaural properties of ICC neurons (Malmierca, 2003).

\subsubsection{Functional role of the ICC}

The ICC is the major auditory integrative center in the central auditory pathway and it is essential for the normal hearing (Jenkins and Masterton, 1982; Winer, 2005). The auditory information from many sources of the brainstem is collected and reorganized into well-ordered spatial and temporal patterns of excitation that can be interpreted by higher centers (Ehert, 1997). The frequency-band laminae containing thousand of neurons with a similar characteristic frequency represent the feature of the ICC organization. It is the structural basis for the frequency-specific arrangements and has caused considerable attention (Winer, 2005). Distinct laminae of the ICC are related to the corresponding sound frequencies. Considerable data have shown evidence that the ICC is associated with the topographical representations of frequency, tone-response latency and threshold, response pattern, sharpness of the frequency tuning, bestmodulation frequency for amplitude-modulated sounds (Ehret, 1997). The ICC receives both excitatory and inhibitory inputs from each ear. Several types of binaural rate/intensity functions (RIFs) have been described in the ICC, which may result from interaction of excitation and inhibition in various degrees (Irvine, 1986). The ICC units are sensitive to interaural time difference (ITDs), interaural intensity difference (IIDs), and interaural phase difference (IPDs) (Irvine, 1986; Wenstrup et al., 1988). IIDs are 
thought to be the principal cue for animals to localize high frequency sounds (Pollak et al., 2003), whereas physical considerations constrain ITD processing to low frequencies. In addition, we all know that most natural sounds including communication sounds contain various frequency and amplitude modulations. These modulations generate a temporal structure which is a major source of information for recognizing sounds (Shannon et al., 1995; Koch and Grothe, 1998). Considerable data have shown that the ICC neurons can also extract the temporal auditory information like the periodicity of amplitude- or frequency-modulated sounds found in complex signals (Burger and Pollak, 1998; Koch and Grothe, 1998, 2000). Therefore, the ICC is the vital integrative center in the central auditory system.

\subsection{External cortex of the inferior colliculs (ICX)}

\subsubsection{Neuronal organization}

The ICX includes the lateral and ventral regions of the IC throughout the extent of the IC. It covers the ICC laterally, ventrally and rostrally (Faye-Lund and Osen, 1985; Malmierca, 1991; Fig. 1.1). The ICX is found to have a cortical cytoarchitecture including three layers (Huffman and Henson, 1990). Layer 1, the fibrous outer layer, is a continuation of the layer 1 of the ICD (see below). The neurons in the layer 1 are small and scattered. Together with the ICD, they form a fibrocellular capsule covering the ICC laterally and dorsally. Layer 2, the small-celled layer, has high acetylcholinesterase and GABA concentrations (rat, Paxinos and Watson, 1998; Paxinos, 1999). Layer 3 constitutes the largest portion of the ICX. It is composed by scattered small, medium and large multipolar neurons. A distinct boundary is formed between the large multipolar cells of the ICX and the small, densely packed neurons of the ICC (Huffman and Henson, 
1990). Malmierca (1991) presented the computer-assisted 3-D reconstruction of the neurons in this region. The results demonstrated that the dendritic arbors of the ICX are quite different from those of the ICC both in the thickness and the orientation.

\subsubsection{Afferent and efferent projections of the ICX}

The ICX receives both the auditory and nonauditory inputs, and may play a role in acousticomotor response (Malmierca, 2003).

Auditory inputs to the ICX: The ICX receives limited ascending projections from the lower auditory brain stem. Its main auditory inputs are from the local connection of the ipsilateral ICC (Huffman and Henson, 1990; Oliver, 2005). Through the CoIC, the ICX also receives some minor projections from the contralateral ICC (Moore and Goldberg, 1963; Willard and Ryugo, 1979; Huffman and Henson, 1990). In addition, the $\mathrm{CN}$, SOC and DNLL also send minor projections to the ICX. Furthermore, the ICX also gets the inputs from the auditory cerebral cortex originating immediately rostral to the primary auditory cortex (Faye-Lund, 1985; Huffman and Henson, 1990). The projections are ipsilateral and terminate in layer 2 and 3. The ICX also receives the projections from the ipsilateral medial division of the MGB (Winer, 2005).

Non-auditory inputs to the ICX: Using anterograde and retrograde transport of horseradish peroxidase (HRP), several studies have demonstrated that the ICX also receives input fibers from several non-auditory structures, which accounts for its role in acousticomotor responses. These somatosensory structures include the cuneate and trigeminal nuclei (Coleman and Clerici, 1987), the lateral nucleus of the substantia nigra (Olazabal and Moore, 1989), the parabrachial region, the midbrain central gray, the periventricular nucleus and the globus pallidus (Coleman and Clerici, 1987; Yasui et al., 
1990). Aitkin et al. (1978, 1981) have given the electrophysiological evidence that the neurons of the ICX respond not only to auditory stimuli, but also to the somatosensory stimuli. These data strongly support the existence of the non-auditory projections to the ICX.

The neurons of the ICX send the fibers to the dorsal and medial divisions of the MGB. These fibers overlap with the projections from the ICC and ICD, especially in the medial division (Malalmierca, 2003). The ICX also sends inputs to the superior colliculus, the cerebellum and the pontine gray matter (Hashikawa, 1983; Hashikawa and Kawamura, 1983; Huffman and Henson, 1990). Besides these projections, the ICX also projects to the ipsilateral ICC. All these connections support the notion that the ICX is the multisensory integrative nucleus.

\subsubsection{Functional role of the ICX}

The ICX is the target of both auditory and considerable nonauditory inputs, and is thought to be multisensory nucleus (Winer, 2005).

Although the ICX does not play a significant role in auditory processing, it is still an auditory driven center because it receives obvious projections from the ICC, a definitely integrative center in the auditory pathway. So the ICX must have some functions for auditory processing. Using in vivo recording methods, Aitkin et al., (1978) found that some ICX neurons in the anesthetized cat were often poorly driven by acoustic stimuli such as pure tone bursts. The responses displayed broad, irregular tuning and were habituated quickly. After repeptitive stimuli, most ICX neurons did not respond until receiving different stimuli (Aitkin et al., 1978, 1981; Huffman and Henson, 1990). 
The ICX also contributes to control of the somatosensory system. In the anesthetized cat, ICX neurons responded to somatosensory stimuli (Aitkin et al., 1978, 1981). These ICX units usually have large surface-receptive fields, most often on the contralateral side of the body (Huffman and Henson, 1990). Moreover, the ICX sends the projections to the pontine gray matter and cerebellum which are associated with motor function. So it is believed that the ICX is involved in multisensory behaviors including the initial stages of sensory integration required for general alerting behaviors (FayeLund, 1985). The ICX is also thought to have some functions in the process of the visceral or emotional responses to acoustic stimuli (Iwada et al., 1986; LeDoux et al., 1988).

In summary, the ICX is a multisensory nucleus that is functionally different from the ICC and ICD. It processes both auditory and tactile information, suggesting a role in multisensory integration.

\subsection{Dorsal cortex of the inferior colliculus (ICD)}

\subsubsection{Neuronal organization of the ICD}

The ICD is the third subnucleus of the IC. It covers the ICC dorsocaudally and dorsomedially. The ICD is composed predominantly by stellate cells. Unlike the ICC, it lacks the obvious fibrodendritic laminae within the structure (Morest and Oliver, 1984; Faye-Lund and Osen, 1985). Like other cortical structures, the ICD is composed of cortical layers. From ventral to dorsal part of the structure, the cell size of the layers progressively increases. Different layers have been defined in different species.

There are three layers in the ICD of the rat (Faye-Lund and Osen, 1985; Huffman and Henson, 1990; Fig. 1.1). Layer 1 is the most superficial layer that is continuous with 
that over the ICX. It is thin $(7 \mu \mathrm{m})$ and relatively cell-sparse. Layer 1 contains the scattered, small and medium-sized neurons with flattened dendritic fields parallel to the surface of the IC. Layer 2 is deeper and slightly thicker. It is characterized by small- and medium-sized, mostly multipolar neurons that intermingle with the layer 1. Collectively, these two layers together constitute about $1 / 3$ of the thickness of the ICD (Malmierca, 2003). Layer 3 comprises about $2 / 3$ of the maximum thickness of the ICD. It contains small-, medium- and large-sized stellate cells. Large multipolar neurons can be found at the border of the layer 3 and ICC. This border is featured by an abrupt change in dendritic orientation. However, defining a sharp functional border between the ICC and the ICD remains problematic (Oliver, 2005). No reversal of the tonotopic map is found at this border. Malmierca (2003) has demonstrated that the ICD neurons are different from the ICC flat cells by computer-assisted 3D reconstructions. However, some neurons with elongated shape still can be found at the border of the ICD and the ICC, which are thought to be the transitional neurons. In addition, the ICD neurons are also characterized by the presence of the nitric oxide, which has a gradient of distribution running from the ICD toward the ICC (Malmierca, 2003). As a neuromodulator, the nitric oxide has been implicated in neuronal plasticity in the rat IC (Malmierca, 2003).

In the cat and human, the ICD has been divided into 4 layers (cat, Morest and Oliver, 1984; human, Geniec and Morest, 1971). The ICD cytoarchitecture of the cat and human is similar to the rat. It has a thin, fibrous superficial layer (Layer 1), thicker layer 2 and 3, and larger and thicker layer 4 (Huffman and Henson, 1990). Layer 4 is considered as the site of convergence for cortical and collicular terminals (Geniec and Morest, 1971; Huffman and Henson, 1990). Golgi studies in the cat by Morest and Oliver (1984) has 
shown that the deeper layers of the ICD continue beneath the pericentral area before they meet the ICC. Fiber staining and retrograde transport studies have demonstrated the border between the ICC and the ICD of the cat and found the transition from the ICC (Oliver et al., 1991; Oliver, 2005).

\subsubsection{Afferent projections of the ICD}

The inputs to the ICD are distinct from those of the other subnuclei of the IC, the ICC and ICX. The ICD receives the majority of descending fibers from the ipsilateral auditory cortex, although the cortical projections to the ICC and ICX have also been demonstrated (Druga and Syka, 1984; Morest and Oliver, 1984; Faye-Lund, 1985; Coleman and Clerici, 1987; Druga et al., 1997). Recently, using retrograde tracer (Fluorogold, Fluororuby, microbeads) injections, Bajo and Moore (2005) studied the morphology and the location of the cortico-colliculus projection neurons. They found that restricted injections in the ICD produced a large number of labeled neurons in the auditory cortex core and also labeled a homogeneous band of smaller cells in the caudal auditory cortex. The cortico-colliculus projections originate from layer $\mathrm{V}$ of the auditory cortex (cat: Winer and Prieto, 2001; Winer, 2005; rat: Weedman and Ryugo, 1996; gerbil: Bajo and Moore, 2005). In the layer V of the auditory cortex, labeled pyramidal neurons were scattered and characterized by tufted dendritic arbors that terminate in layer 1 of the ICD. In addition, the somata of labeled pyramidal cells in auditory cortex core were larger than those in caudal auditory cortex (Bajo and Moore, 2005). Experimental evidence also indicates that at least two types of layer $\mathrm{V}$ pyramidal neurons, tufted and untufted, participate in the projections (Hefti and Smith, 2000; Bajo and Moore, 2005). Restricted anterograde tracer (dextran biotin) injections in the core and caudal auditory 
cortex generated labeled fibers and terminals in the IC. The main plexuses were located in the ipsilateral ICD. Labeling in the contralateral ICD was very sparse $(5 \%$ of the ipsilateral labeling), with few labeled terminals (Bajo and Moore, 2005).

The ICD also receives significant intrinsic projections from the ipsilateral ICC and commissural inputs from the contralateral ICC (Aitkin and Phillips, 1984; Huffman and Henson, 1990; Saldaña and Merchán, 1992; Merchan and Saldana, 2005). To study the organization of the intrinsic and commissural connections of the rat IC, Saldaña and Merchán (1992) injected the anterograde tracer PHA-L into the ICC. They found that an ipsilateral medial plexus (iMP) formed by axons from the discrete ICC deposit. It extended dorsomedially and ventrolaterally from the injection stie, parallel to the fiberdendritic laminae of the ICC, to the ipsilateral ICD. In addition, efferent fibers from the injection site of the laminated ICC also traverse the commissure of the IC and terminate ventrally in the contralateral ICD (Huffman and Henson, 1990; Saldaña and Merchán, 1992). Aitkin and Phillips (1984) suggest that these contralateral projections are tonotopically organized and associated with an isofrequency contour that complements the tonotopic origin of the opposite ICC. These connections were common to all experiments with different injection sites in the ICC. But the different thickness, position and orientation of the plexus related to the injection sites are also noticed (Saldaña and Merchán, 1992). Furthermore, similar projections are found in the IC of mouse (Frisina et al., 1997), gerbil (Cant, 2005), guinea pig (Malmierca et al., 1995), cat (Malmierca et al., 1998), and bat (Vater and Feng, 1990).

In addition, the ICD also receives ascending projections from the lower auditory brainstem nuclei, including the cochlear nuclei $(\mathrm{CN})$ and the LL (Coleman and Clerici, 
1987; Huffman and Henson, 1990; Cant, 2005; Schofield, 2005). Although the main target of the cochlear nuclei in the IC is the central nucleus, both the ventral and dorsal cochlear nuclei also send projections to the deep layers of the ICD. Like those to the ICC, these projections appear to be topographical organized (Oliver, 1984). But the terminations of such projections appear to be sparser than those to the ICC (Oliver, 1984; Coleman and Clerici, 1987; Zook and Casseday, 1987; Cant, 2005). In addition, Oliver (1987) showed that the projections from the ventral cochlear nucleus arborized more widely than those from the dorsal cochlear nucleus. The ICD receives projections from the VNLL. These inputs are diffuse, widespread and almost exclusively ipsilateral (Whitley and Henkel, 1984). They course in the lateral lemniscus, pass through the INLL and DNLL, and terminate in the ICD. The INLL also sends projections to the ICD. The fibers are largely ipsilateral and terminate diffusely in the deep layers of the dorsal cortex (Whitley and Henkel, 1984; Schofield, 2005). Most DNLL neurons send projections to the ipsilateral or contralateral deep layers of the ICD. The projections terminate more diffusely ipsilaterally (Shneiderman et al., 1988; Schofield, 2005).

\subsubsection{Efferent projections of the ICD}

The targets of efferent projections of the ICD include the medial geniculate body (MGB) and other subdivisions of the IC (intrinsic and commissural connections) (Wenstrup, 2005). Calford and Aitkin (1983) studied the sources of the ascending inputs to the MGB using the retrograde transport of HRP. HRP injections were made iontophoretically through micropipettes that can also be used to record physiological properties at the injection sites. They found that the ICD sent the projections to the dorsal division of the MGB. The MGB then sends ascending inputs to the cortical area 36 and 
the basal ganglia. The results are supported by the later studies (Faye-Lund, 1985; Coleman and Clerici, 1987; Huffman and Henson, 1990). The ICD also sends the intracolliculus projections to the ipsilateral and/or contralateral ICC (Saldana and Merchan, 2005). A PHA-L injection into the central ICD led to sparse labeling in the ICC on both sides. The labeled fibers produced a medial and a lateral laminar plexus in each IC. The plexus spanned the IC rostrocaudally and caudally at their ventral ends. The organization of the ICD intracollicular connections accentuates the connectional parallels between the ICC and the ICD.

\subsubsection{Functional role of the ICD}

The functional role of the ICD in hearing is still unknown (Winer and Schreiner, 2005). As previously described, frequency laminae are absent in the ICD, but the neurons do form several layers. Therefore, further levels of functional organization may exist (Winer et al., 1998). The tonotopic map of the ICC extends dorsomedially into the deeper layers of the ICD, where neurons have similar response properties to the ICC neurons. In addition, the ICD sends some projections to the ICC that is the major integration center in the auditory pathway, so the ICD may contribute to the auditory attention and modulate the subject's attention to the stimuli (Aitkin et al, 1975). By contrast, neurons in the superficial layers of the ICD are like those in the ICX. They are tuned broadly (Aitkin et al., 1994). ICD neurons are poorly driven by the acoustic stimuli and tend to habituate quickly (Merzenich and Reid, 1974; Aitkin et al., 1975). These neurons respond primarily to stimulus onset and thereafter display erratic firing patterns. Limited behavioral results indicate that damage to the dorsal cortex can affect attention and vigilance more severely than auditory discrimination behavior (Jane et al., 1965; Winer, 
2005). Accordingly, the response properties and connections of the ICD neurons suggest that they may act as a modulator of the auditory information processing mainly performed by the ICC neurons (Winer, 2005).

\subsection{Synaptic receptors in the IC}

Synaptic receptors in IC neurons are activated by excitatory and inhibitory amino acids, including glutamate, $\gamma$-aminobutyric acid (GABA) and glycine. Other neuroactive substances are also released onto IC neurons, i.e., serotonin and norepinephine. I will emphasize, here, the physiological properties of glutamate and GABA receptors.

\subsubsection{Glutamate receptors}

Glutamate has been considered the most important excitatory substance in the brain that fulfills most of criteria for the neurotransmitter candidate. Based on distinct pharmacology and physiology, various glutamate effects can be mediated by two main categories of receptors: ionotropic glutamate receptors (iGluRs) and metabotropic glutamate receptors (mGluRs) (Hollmann and Heinemann, 1994). IGluRs are coupled directly to cationic channels. They include three types of receptors: alpha-amino-3hydroxy-5-methyl-4-isoxazolepropionate (AMPA), kainate and N-methyl-D-aspartate (NMDA) receptors. MGluRs do not form functional ion channels but exert their effects by G-protein and different intracellular systems (Tanable et al., 1992). My emphasis, here, is on the contribution of iGluRs to synaptic transmission in the IC.

\subsubsection{AMPA receptors}

A variety of previous studies have demonstrated that AMPA receptors mediate fast excitatory synaptic neurotransmission in most of the synapses in the CNS (Parks, 2000). The AMPA receptor family includes four related subunits, GluR1 to GluR4 
(Boulter et al., 1990; Seeburg, 1993; Hollmann and Heinemann, 1994). These four subunits have similar size and share $68-73 \%$ homology in their amino acid sequence. In addition, they all contain four distinct hydrophobic domains (M1-M4). AMPA is the most potent selective agonist causing a fast desensitization of the receptors (Hollmann and Heinemann, 1994).

AMPA receptors have been found throughout the CNS including the IC (Parks, 2000), although there are regional differences in the distribution. In the rat IC, using in situ hybridizafion, Sato et al., (1993) found low levels of GluR1 and 4 and high levels of GluR 2 and 3 mRNA expressions. Expression of GluR1-4 proteins is also demonstrated in the IC. An immunocytochemical study by Petralia and Wenthold (1992) found moderate to high levels of expression of GluR $1,2 / 3 / 4 \mathrm{c}$ and GluR 4 proteins in the rat IC. Similar results were verified in recent studies (Gaza and Ribak, 1997; Caicedo and Eybalin, 1999).

Intracellular and patch clamp recordings from ICC neurons in brain slices of mouse, rat, and gerbil with the stimulation of the lateral lemniscus (LL) or the IC commissure (CoIC) have demonstrated that AMPA receptors are involved in the excitatory synaptic neurotransmission (Wagner, 1996; Moore et al., 1998; Ma et al., 2002). The early component of excitatory postsynaptic potentials (EPSPs) can be blocked by the AMPA antagonists such as CNQX, NBQX without eliminating the latter response (Ma et al., 2002). These results indicate that AMPA receptors mediate the early part of the EPSPs. A more accurate assement of the kinetics of these EPSPs was made when inhibitory responses were blocked by the GABA and glycine receptor antagonists. AMPA receptor-mediated EPSPs have short rise time and decay times (means: 5.4 and 
$35.6 \mathrm{~ms}$, respectively). Moreover, the current-voltage relationship for AMPA receptormediated EPSPs was linear (Ma et al., 2002). This result shows that the AMPA receptormediated responses are not voltage-dependent. In addition, the AMPA receptor-mediated response was not affected by extracellular $\mathrm{Mg}^{2+}$ concentration. Therefore, the propeties of AMPA receptor-mediated responses in IC neurons are similar to those of AMPA receptor-mediated responses recorded from neurons in other regions of the auditory system (Parks, 2000).

Intracellular recordings from ICD neurons in the coronal brain slices from 21-50 days old rats found that AMPA receptors contribute to the EPSPs elicited by stimulation of the CoIC (Smith, 1992). Using same techniques, Li et al., (1999) reported the similar results from the IC brain slice of adult rats. Synaptic responses were generally observed as a complex mix of inhibitory postsynaptic potentials (IPSPs) and EPSPs by stimulation of the CoIC. Isolated EPSPs contained a short-latency component that could be blocked by CNQX or DNQX. Their data indicate that AMPA receptors mediate the short-latency EPSPs in the ICD neurons. Whether these AMPA responses can also be evoked by stimulating other inputs such as the ICC or the LL requires further studies.

In vivo electrophysiological studies also demonstrate that AMPA receptors contribute to excitatory responses to tones or other acoustic stimuli. Iontophoretic application of NBQX, a selective AMPA receptor antangonist, decreased or even blocked excitatory responses to contralaterally presented tone bursts in most ICC neurons (Zhang and Kelly, 2001, 2003). AMPA receptors also contribute to responses evoked by acoustic stimulation at all sound pressure levels above threshold. Application of AMPA receptor antagonists reduced the firing rate of the IC neurons throughout their dynamic range 
(Zhang and Kelly, 2001; Kelly and Zhang, 2002; Kelly and Caspary, 2005). In addition, blockade of AMPA receptors depressed the activity of most ICC neurons in response to sinusoidally amplitude-modulated tones across a wide range of modulation frequencies (Zhang and Kelly, 2003).

\subsubsection{NMDA receptors}

NMDA receptors also belong to the family of iGluRs, while they act in different ways from the AMPA receptors in mediating excitatory neurotransmission. Compared to AMPA receptors, their activation and deactivation kinetics are much slower. NMDA receptors have several unique properties (Kandel et al, 2000). Activation of NMDA receptors is affected by the concentration of the extracellular $\mathrm{Mg}^{2+}$ in a voltage-dependent manner (Nowak et al., 1984; Mayer and Westbrook, 1987). Glycine-binding site occupancy is needed for the activation of NMDA receptors. In addition, the channel is highly permeable to $\mathrm{Ca}^{2+}$. NMDA receptor has three different subunit families: NR1, 2AD and NR3A-B (Monyer et al., 1992; Das et al., 1998; Chatterton et al., 2002). It is thought that several properties of NMDA receptors such as channel kinetics, voltagedependence and sensitivity of $\mathrm{Mg}^{2+}$ blockade are determined by NR2 subunits incorporated into the NMDA receptor complex (Monyer et al., 1992; Vicini et al., 1998; Wyllie et al., 1998; Behe et al., 1999). NR1 with at least one of the NR2 subunits form a functional NMDA receptor (Sheng et al., 1994).

Recordings from ICC neurons in the brain slices have demonstrated that NMDA receptors are involved in synaptic responses evoked by stimulation of the LL or the CoIC (Smith, 1992; Wagner, 1996; Li et al., 1998, 1999; Moore et al., 1998; Ma et al., 2002;

Wu et al., 2004). The late component of the EPSPs evoked by electrical stimulation of 
the LL could be selectively blocked by the NMDA receptor antagonist, APV or CPP (Ma et al., 2002). This result indicates that the late component of the EPSPs is mediated by NMDA receptors. The kinetics of NMDA receptor-mediated EPSPs are different from those of AMPA receptor-mediated EPSPs. The rise time and decay time of NMDAEPSPs (22.8 and $92.1 \mathrm{~ms}$, respectively) are longer than those of AMPA-EPSPs (Ma et $a l .$, 2002). In addition, NMDA receptor-mediated EPSPs could be greatly enhanced by removing extracellular $\mathrm{Mg}^{2+}$, suggesting that $\mathrm{Mg}^{2+}$ can suppress activation of NMDA receptors. The current-voltage $(\mathrm{I} / \mathrm{V})$ relationship for the NMDA receptor-mediated response was nonlinear, indicating that NMDA receptor-mediated EPSPs are voltagedependent. Moreover, it is very interesting to notice that NMDA receptors in the ICC neurons are different from NMDA receptors in several other regions, for example, the hippocampal neurons. NMDA receptor-mediated EPSPs in the ICC can be easily elicited at the cell's resting membrane potential and even after blocking AMPA receptormediated responses (Ma et al., 2002; Wu, 2005). The possible explanation is that NMDA receptors in IC neurons may have lower sensitivity to $\mathrm{Mg}^{2+}$ blockade, so activation of the receptor does not require much membrane deploarization. Furthermore, the lower sensitivity to $\mathrm{Mg}^{2+}$ of NMDA receptors in the IC may be attributed to the subunit composition of the NMDA receptors. In ICC neurons, NR2C and NR2D subunits may be expressed more than NR2A and NR2B subunits (Wu, 2005). However, further studies are needed to understand the relationship between the receptor subunit composition and $\mathrm{Mg}^{2+}$ sensitivity and voltage dependence of NMDA receptors in the IC.

In vivo studies also have provided the evidence that NMDA receptors are involved in the synaptic excitation (Faingold et al., 1989, 1991; Feldman and Knudsen, 
1994; Zhang and Kelly, 2001; Kelly and Zhang, 2002; Kelly and Caspary, 2005). In vivo single unit recordings from cells in the ICC clearly proved the involvement of glutamate (Faingold et al., 1989, 1991). Ionophoretic application of NMDA increased acoustically evoked firing. Furthermore, the selective NMDA receptor antagonist had a blocking effect on most ICC neurons during the late period of a sustained response to tone bursts (Zhang and Kelly, 2001, 2003). Kelly and Zhang (2002) also demonstrated that NMDA receptors could mediate responses at low to moderate levels of acoustic stimulation. In addition, NMDA receptors contribute to the responses to sinusoidally amplitudemodulated tones in ICC neurons (Zhang and Kelly, 2003).

The functional role of NMDA receptors in IC neurons is far from fully understood. From the results mentioned above, we have known that NMDA receptors are involved in generating excitatory effects in the postsynaptic neurons of the ICC. The time course of NMDA receptor-mediated action is long because of the slow kinetics of the receptor. This, therefore, may provide a wide window for processing the inputs coverging onto IC neurons (Kelly and Zhang, 2002; Wu, 2005). In addition, the primary role of NMDA receptors may not be only to generate the excitatory response. Instead they seem to be involved in neural plasticity. Activation of NMDA receptors was required for generation of long-term potentiation in ICC neurons (Zhang and $\mathrm{Wu}, 2000$; $\mathrm{Wu}$ et al., 2002). NMDA receptors are also thought to be related to audiogenic seizures. Removing extracellular $\mathrm{Mg}^{2+}$ or application of NMDA can induce sustained or epileptiform activity in the IC neurons (Smith, 1992; Wagner, 1996; Li et al., 1998, 1999).

\subsection{2 $\gamma$-Aminobutyric acid (GABA) receptors}


GABA is the major inhibitory neurotransmitter in CNS. It plays a key role in modulating excitatory activity of neurons. In the auditory system, GABAergic inhibition has vital functions in auditory information processing. It has been demonstrated that GABAergic inhibition can modulate the firing rate, tuning curve, duration tuning and binaural response of auditory neurons (Faingold et al., 1989; Yang et al., 1992; Casseday et al., 1994; Park and Pollak, 1994; Klug et al., 1995; Fuzessery and Hall, 1996; Burger and Pollak, 1998; Koch and Grothe, 1998; Ingham and McAlpine, 2005; Wu, 2005). In addition, GABA-mediated inhibition is also related with audiogenic seizure genesis (Faingold, 2002, 2005). GABA mediates its inhibitory actions via distinct receptor systems including ionotropic $\mathrm{GABA}_{\mathrm{A}}$ and metabotropic $\mathrm{GABA}_{\mathrm{B}}$ receptors (Bowery, 1993; Bettler et al., 2004; Takeda et al., 2004). Activation of $\mathrm{GABA}_{\mathrm{A}}$ receptors induces rapid transmission, while $\mathrm{GABA}_{\mathrm{B}}$ receptor mediates a slow response linked to the binding and activation of G-proteins (Bowery, 1993; Bowery et al., 2002; Bettler et al., 2004). The available data have shown that both $\mathrm{GABA}_{A}$ and presynaptic $\mathrm{GABA}_{\mathrm{B}}$ receptors can modulate the auditory processing in IC neurons (Smith, 1992; Moore et al., 1998; Ma et al., 2002; Wu et al., 2004; Sun et al., 2006).

\subsubsection{1 $\mathrm{GABA}_{\mathrm{A}}$ receptor}

The $\mathrm{GABA}_{\mathrm{A}}$ receptor is a heteromeric pentamer related to the super-family of fast, ion channel receptors. It is composed of five subunits. Currently, there are at least $6 \alpha, 4 \beta, 4 \gamma, 1 \delta$, and $2 \rho$ subunits found (Barnard et al., 1998). The subunit construction of the $\mathrm{GABA}_{\mathrm{A}}$ receptor will partly determine the properties of the receptor response. The receptor containing $\alpha 2, \beta$ and $\gamma$ subunits has greater efficacy for GABA than the receptor containing $\alpha 1$ subunit (Shiraishi et al., 2001). The $\beta$ subunits may contribute to the 
control of channel gating and agonist binding sites (Verdoon et al., 1990). The $\gamma$ subunits with $\alpha$ and $\beta$ subunits can affect the single channel conductance (Angelotti and Macdonald, 1993). In vivo, the $\mathrm{GABA}_{\mathrm{A}}$ receptor complex is thought to be composed of $\alpha, \beta$, and $\gamma$ subunits (Rabow et al, 1995). In the IC, studies using selective receptor ligands have shown the presence of $\mathrm{GABA}_{\mathrm{A}}$ receptors in IC neurons (Bristow and Martin, 1988; Caspary et al., 1990). Using the quantitative in situ hybridization techniques, Shiraishi et al., (2001) showed heavy labeling for $\mathrm{GABA}_{\mathrm{A}}$ receptor $\alpha 1$ and $\gamma 2 \mathrm{~L}$ subunits and lower labeling of $\mathrm{GABA}_{\mathrm{A}}$ receptor $\alpha 2$ and $\gamma 2 \mathrm{~S}$ subunits.

$\mathrm{GABA}_{\mathrm{A}}$ receptors are involved in the synaptic neurotransmission in IC neurons. Intracellular and patch clamp recordings from brain slices of rats and gerbils have demonstrated that inhibitory postsynaptic potentials (IPSPs) of ICC neurons are predominantly mediated by $\mathrm{GABA}_{\mathrm{A}}$ receptors (Smith, 1992; Moore et al., 1998; Ma et al., 2002; Wu et al., 2004). Similar to the AMPA receptor-mediated EPSCs, GABA receptor-mediated IPSCs had fast rise time (less than $10 \mathrm{~ms}$ ), decay time (about $30 \mathrm{~ms}$ ) and a half-width (20 to $30 \mathrm{~ms}$ ) (Kraushaar and Backus, 2002; Wu, 2005). The fast responses mediated by $\mathrm{GABA}_{\mathrm{A}}$ receptor make it possible for IC neurons to integrate excitatory and inhibitory synaptic inputs arriving simultaneously (Wu, 2005). Blocking $\mathrm{GABA}_{\mathrm{A}}$ receptor-mediated inhibition enhanced the excitatory responses mediated by both AMPA and NMDA receptors, and prolonged the time course of the NMDA receptormediated responses. In addition, eliminating $\mathrm{GABA}_{\mathrm{A}}$ receptor-mediated inhibition could depolarize an ICC neuron over a relative long time (hundreds of $\mathrm{ms}$ ) and generate epileptic-like discharges (Ma et al., 2002; Wu et al., 2004; Wu, 2005). 
In vivo studies have also demonstrated the importance of $\mathrm{GABA}_{\mathrm{A}}$ receptor in regulating physiological responses of IC neurons to acoustic stimulation. Studies in bat, chinchilla and guinea pig have shown that $\mathrm{GABA}_{\mathrm{A}}$ receptors in the IC could induce changes in a neuron's receptive field (frequency tuning curve) (Yang et al., 1992; Palombi and Caspary, 1996; Kelly and Caspary, 2005). After application of the GABA receptor antagonists, the neuron displayed a significant change of firing rate within the excitatory response area. In addition, local blockade of $\mathrm{GABA}_{\mathrm{A}}$ receptors in the IC could shape binaural responses (Zhang et al., 1999; Kelly and Caspary, 2005). $\mathrm{GABA}_{\mathrm{A}}$ receptor antagonist, bicuculline, increased the firing rate and the selectivity of responses to binaural inputs. Bicuculline also substantially increased the discharge rates of IC neurons to modulated sounds. Moreover, blocking $\mathrm{GABA}_{\mathrm{A}}$ receptors often abolished the duration selectivity of IC neurons (Chen, 1998; Brand et al., 2000; Casseday et al., 2002). All of these results indicate the important function of $\mathrm{GABA}_{\mathrm{A}}$ receptors in auditory information processing (Kelly and Caspary, 2005).

\subsubsection{2 $\mathrm{GABA}_{\mathrm{B}}$ receptor}

\subsection{Molecular structure of $\mathrm{GABA}_{\mathrm{B}}$ receptor}

Similar as the structure of mGluRs, the $\mathrm{GABA}_{B}$ receptor has 7-transmembrane domains, an intracellular C-terminus and a long extracellular N-terminus containing leucine isoleucine valine binding protein (LIV-BP), which is possible GABA binding site. The receptor is demonstrated to exist as a heterodimer with subunits $\mathrm{GABA}_{\mathrm{B} 1}$ and $\mathrm{GABA}_{\mathrm{B} 2}$ (Kaupmann et al., 1997; Jones et al., 1998; Kuner et al., 1999; Ng et al., 1999). The $\mathrm{GABA}_{\mathrm{B} 1}$ subunit is necessary for agonist activation, while the $\mathrm{GABA}_{\mathrm{B} 2}$ protein serves to escort $\mathrm{GABA}_{\mathrm{B} 1}$ to the cell surface and appears to be the receptor component 
that links to the G protein (Calver et al., 2001; Calvez et al., 2001; Pagano et al., 2001; Bowery et al., 2002). Therefore, it is thought that the agonist will bind to the GABA subunit and lead a conformational change in the protein system that allows $\mathrm{GABA}_{\mathrm{B} 2}$ to engage and activate the $\mathrm{G}$ protein system.

The GABA $\mathrm{B}_{1}$ subunit has $a, b, c, d$ and $e 5$ splice variants. Among them, the $\mathrm{GABA}_{\mathrm{B} 1 \mathrm{a}}$ and $\mathrm{GABA}_{\mathrm{B} 1 \mathrm{~b}}$ are the most abundant isoforms and are the only variants that are highly conserved among different species (Bettler et al., 2004). GABA $\mathrm{B}_{\mathrm{a}}$ and $\mathrm{GABA}_{\mathrm{B} 1 \mathrm{~b}}$ show difference in their distribution in the CNS (Benke et al., 1999; Billinton et al., 1999; Bischoff et al., 1999; Fritschy et al., 1999). Studies with cerebellum and spinal cord of rat and human suggest that $\mathrm{GABA}_{\mathrm{B} 1 \mathrm{a}}$ is located predominantly at presynaptic terminals, while $\mathrm{GABA}_{\mathrm{B} l \mathrm{~b}}$ is present at postsynaptic sites (Kaupmann et al., 1998; Billinton et al., 1999; Bischoff et al., 1999; Towers et al., 2000; Princivalle et al., 2002). However, in other brain regions, $\mathrm{GABA}_{\mathrm{Bla}}$ is found at postsynaptic membrane and $\mathrm{GABA}_{\mathrm{B} 1 \mathrm{~b}}$ protein is associated with presynaptic terminals (Benke et al., 1999; Princivalle et al., 2002). Using immunohistochemical methods, Porkhalkali et al., (2000) suggest that $\mathrm{GABA}_{\mathrm{B} 1 \mathrm{~b}}$ can be found at both pre and postsynaptic sites. Therefore, the issue of $\mathrm{GABA}_{\mathrm{B} 1 \mathrm{a}}$ and $\mathrm{GABA}_{\mathrm{B} I \mathrm{~b}}$ respectively representing pre- and postsynaptic subtypes requires further detailed immunohistochemical analysis at electronic level. A number of studies did not find significant difference of the pharmacology between $\mathrm{GABA}_{\mathrm{B} 1 \mathrm{a}}$ and GABA $_{\mathrm{B} 1 \mathrm{~b}}$ subunits (Malitschek et al., 1998; Braunner-Osborne et al., 1999). Both $\mathrm{GABA}_{\mathrm{B} 1 \mathrm{a}}$ and $\mathrm{GABA}_{\mathrm{B} 1 \mathrm{~b}}$ can couple to $\mathrm{K}^{+}$and $\mathrm{Ca}^{2+}$ channels (Filippov et al., 2000).

With respect to $\mathrm{GABA}_{\mathrm{B} 2}$ subunit, three transcripts have been identified including $\mathrm{GABA}_{\mathrm{B} 2} \mathrm{a}, \mathrm{b}$ and $\mathrm{c}$ (Clark et al., 2000). These variants differ in the extreme C terminal 
regions, which does not affect the interaction of $\mathrm{GABA}_{\mathrm{B} 1}$ and $\mathrm{GABA}_{\mathrm{B} 2}$, and hence heterodimer formation.

\subsection{Distribution of $\mathrm{GABA}_{\mathrm{B}}$ receptor in the $\mathrm{IC}$}

The distribution of $\mathrm{GABA}_{\mathrm{B}}$ heterodimer component proteins or encoding mRNAs, $\mathrm{GABA}_{\mathrm{B} 1}$ and $\mathrm{GABA}_{\mathrm{B} 2}$, is widespread throughout the CNS of various vertebrate species (Billinton et al., 1999; Calver et al., 2000; Fritschy et al., 2004), which correlates well with receptor binding studies (Bowery et al., 1987; Chu et al., 1990). In the rat brain, mRNAs encoding $\mathrm{GABA}_{\mathrm{B} 1}$ are detectable in almost all neuronal populations. The highest density of $\mathrm{GABA}_{\mathrm{B} 1}$ mRNA is expressed in hippocampus, thalamic nuclei, and cerebellum. $\mathrm{GABA}_{\mathrm{B} 2}$ mRNA is abundant in the piriform cortex, hippocampus, thalamus, medial habenula and all layers of the cortex (Jones et al., 1998; Durkin et al., 1999; Berthele et al., 2001). The $\mathrm{GABA}_{\mathrm{B} 1}$ and $\mathrm{GABA}_{\mathrm{B} 2}$ mRNA expression is mostly overlapping, which qualitatively parallel those of $\mathrm{GABA}_{\mathrm{B}}$ agonist and antagonist binding sites (Kaupmann et al., 1998; Bischoff et al., 1999). In general, heteromeric $\operatorname{GABA}_{\mathrm{B}(1,2)}$ receptor constitutes the majority of $\mathrm{GABA}_{\mathrm{B}}$ binding sites, but the subunits $\mathrm{GABA}_{\mathrm{B} 1}$ and $\mathrm{GABA}_{\mathrm{B} 2}$ also can form receptors independent of each other.

Several studies have shown the presence of $\mathrm{GABA}_{\mathrm{B}}$ receptor in the IC (Bowery et al., 1987; Chu et al., 1990; Fritschy et al., 2004). But detailed information about distribution of $\mathrm{GABA}_{\mathrm{B}}$ receptors within the IC is very limited. Milbrandt et al., (1994) used quantitative receptor autoradiography to assess $\mathrm{GABA}_{\mathrm{B}}$ receptor binding sites. They found that $G A B A_{B}$ receptor binding was significantly higher in the ICD than in the ICC and ICX in the Fischer 344 rat brain. Similar findings were reported by Fubara et al (1996). They used ${ }^{3} \mathrm{H}-\mathrm{GABA}$ in the presence of isoguvacine, a selective GABA receptor 
agoinst, to block $\mathrm{GABA}_{\mathrm{A}}$ receptor sites. Their results indicated that the distribution of $\mathrm{GABA}_{\mathrm{B}}$ receptor sites within the IC resembled that for $\mathrm{GABA}_{\mathrm{A}}$ receptors. The area of the highest density of $\mathrm{GABA}_{\mathrm{B}}$ receptor sites was in the dorsomedial part of the IC. And the density was uniformly low throughout the remainder of the IC. The underlying mechanism for the difference in $\mathrm{GABA}_{\mathrm{B}}$ distribution within the IC is not known. Moreover, these studies were unable to determine if the difference is at pre- and/or postsynaptic sites. Further experiments using ultrastructural immunocytochemical techniques are needed to investigate pre- and postsynaptic distribution of $\mathrm{GABA}_{\mathrm{B}}$ receptors in the IC. However, on the whole, these studies confirm the presence of $\mathrm{GABA}_{\mathrm{B}}$ receptors in the $\mathrm{IC}$ and that the most prominent expression of $\mathrm{GABA}_{\mathrm{B}}$ receptors is in the ICD.

\subsection{Presynaptic $\mathrm{GABA}_{\mathrm{B}}$ receptor}

It is widely believed that the $\mathrm{GABA}_{\mathrm{B}}$ receptors are present on both pre- and postsynaptic sites. Presynaptic $\mathrm{GABA}_{\mathrm{B}}$ receptors are subdivided into autoreceptors and heteroreceptors on the basis of their function. Autoreceptors can control GABA release, while heteroreceptors can inhibit all other neurotransmitter release.

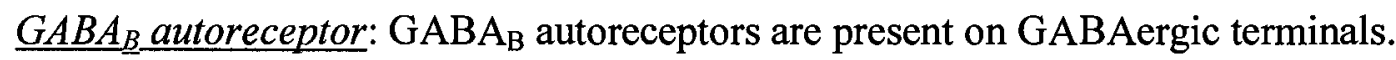
Because of the low affinity of $\mathrm{GABA}_{\mathrm{B}}$ receptors for $\mathrm{GABA}$ and far localization of the receptors from the synaptic cleft, $\mathrm{GABA}_{\mathrm{B}}$ autoreceptors are only activated after synchronized activation of GABAergic neurons (Lambert and Wilson, 1993). However, once activated by synaptically released $G A B A, G A B A_{B}$ receptors can regulate $\mathrm{GABA}$ release. Previous studies have shown that $\mathrm{GABA}_{\mathrm{B}}$ autoreceptors inhibit GABA release by inhibiting presynaptic $\mathrm{Ca}^{2+}$ entry into terminal release sites (Otis and Mody, 1992) 
through a pertussis toxin-sensitive signal transduction cascade (Potier and Dutar, 1993; Calver et al., 2002). Whether this happens by activation of potassium channels or inhibition of high voltage-activated $\mathrm{Ca}^{2+}$ channels in the presynaptic terminals is still a dispute topic. The effects of presynaptic $\mathrm{GABA}_{B}$ autoreceptors on $\mathrm{GABA}_{A}$ receptormediated responses have been demonstrated in the rat ICC (Ma et al., 2002). Using whole-cell patch recording techniques, Ma et al., (2002) found that GABA $\mathrm{B}$ receptor agonist, baclofen, did not affect the cell membrane conductance and the rate of firing. However, baclofen decreased the amplitude of the inhibitory postsynaptic potentials. This effect could be completely blocked by the $\mathrm{GABA}_{\mathrm{B}}$ receptor antagonist, phaclofen. These results indicated that presynaptic $\mathrm{GABA}_{\mathrm{B}}$ autoreceptors depressed the GABAergic inhibition in ICC neurons. The effects of GABA $\mathrm{B}_{\mathrm{B}}$ autoreceptors in the ICD and the ICX are to be determined.

$\underline{G A B A_{B}}$ heteroreceptor: $\mathrm{GABA}_{\mathrm{B}}$ heteroreceptors are widely distributed throughout the CNS and they can control the release of numerous neurotransmitters including glutamate, monoamines and several neuropeptides (Bonanno and Raiteri, 1993; Lei and McBain, 2003). Pharmacological difference may occur not only between auto- and heteroreceptors but also between heteroreceptors modulating the release of different neurotransmitters (Phelan, 1999). Usually, higher concentrations of baclofen are required to depress transmitter release via heteroreceptors than via autoreceptors (Lei and McBain, 2003). The reason for this difference is still unclear. But the possible explanation is the difference between two groups of $\mathrm{GABA}_{\mathrm{B}}$ receptors in the signal transduction pathways coupling the receptor to the effector (Calver et al., 2002). It has also been suggested that $\mathrm{GABA}_{\mathrm{B}}$ receptors may modulate spontaneous and evoked release of glutamate through 
different cellular mechanism (Wu and Saggau, 1997). To study whether activation of presynaptic $\mathrm{GABA}_{\mathrm{B}}$ receptor can regulate glutamatergic excitation, Sun et al., (2006) examined the effects of baclofen on excitatory postsynaptic currents (EPSCs) evoked by stimulation of the lemniscal inputs to the ICC neurons. We confirmed that baclofen can significantly reduce the glutamatergic excitation in the ICC neurons.

The results of autoradiographic studies indicate that $\mathrm{GABA}_{\mathrm{B}}$ receptors are distributed more in the ICD than the ICC (Chu et al., 1990; Milbrandt et al., 1994; Fubara et al., 1996). However, physiological role of $\mathrm{GABA}_{\mathrm{B}}$ receptors in the ICD has not been known. Whether presynaptic $\mathrm{GABA}_{\mathrm{B}}$ receptors in $\mathrm{ICD}$ neurons have the same functions in modulating transmitter release as those in ICC neurons requires further investigation.

\subsection{Postsynaptic $\mathrm{GABA}_{\mathrm{B}}$ receptor}

Turning to postsynaptic $\mathrm{GABA}_{\mathrm{B}}$ receptors, there is an extensive literature demonstrating that application of baclofen can cause a postsynaptic hyperpolarization of most of the principal and interneuron population in the CNS (Calver et al., 2002). Furthermore, slow inhibitory postsynaptic currents (IPSCs) can be induced by stimulation of GABAergic afferents in some neuronal populations (Mott et al., 1999; Liu and Leung, 2003). The biophysical characteristics of the IPSCs are similar to those of the baclofeninduced hyperpolarization (Davies et al., 1990; Cruneli and Leresche, 1991). The kinetics of the IPSCs are consistent with general time course of metabotropic receptor induced physiological events. The long time course of the IPSCs reflects G-protein coupling, the duration of GABA occupancy in $\mathrm{GABA}_{\mathrm{B}}$ receptor and the rate of GTP hydrolysis (Otis et al., 1993; Calver et al., 2002). GABA B $_{\mathrm{B}}$ receptor-induced inhibition is different from $\mathrm{GABA}_{\mathrm{A}}$ receptor-mediated inhibitory responses. $\mathrm{GABA}_{\mathrm{A}}$ responses provide inhibition 
primarily via membrane shunting, while $G_{A B A}$ responses produce inhibition via membrane hyperpolarization that is thought to be enough to reduce the firing probability and prevent activation of NMDA receptors by maintaining the voltage-dependent magnesium block of the NMDA receptor channel (Morrisett et al., 1991). It is interesting to note that $\mathrm{GABA}_{\mathrm{B}}$ receptors are usually located in perisynaptic or extrasynaptic areas (Kulik et al., 2003; Frritschy et al., 2004). Thus, it is generally accepted that GABA receptor-mediated IPSCs are most likely to be generated physiologically only when GABAergic interneurons are synchronized activated.

The question of whether postsynaptic $\mathrm{GABA}_{B}$ receptors are involved in synaptic transmission in the IC remains unsettled. The $\mathrm{GABA}_{\mathrm{B}}$ receptor agonist, baclofen, did not increase the cell membrane conductance and alter the rate of firing of the ICC neurons (Ma et al., 2002). In addition, baclofen did not affect the resting membrane potential and $\mathrm{K}^{+}$conductance of ICC neurons (Hosomi et al., 1997). These negative results provide no evidence of the presence of postsynaptic $\mathrm{GABA}_{\mathrm{B}}$ receptors. However, the results that activation of $\mathrm{GABA}_{\mathrm{B}}$ receptors exerts inhibitory effects on GABAergic inhibition and glutamatergic excitation suggest that the modulatory influence of $\mathrm{GABA}_{\mathrm{B}}$ receptors on ICC neurons is via presynaptic mechanisms. Based on the results of autoradiographic experiments, $\mathrm{GABA}_{\mathrm{B}}$ receptors are distributed more in the dorsal part compared to the central part of the IC. Therefore, futher investigations are needed to test the role of postsynaptic $\mathrm{GABA}_{\mathrm{B}}$ receptors in ICD neurons.

\subsection{Functional role of $\mathrm{GABA}_{\mathrm{B}}$ receptors in the IC}

Regardless of presynaptic or postsynaptic location, $\mathrm{GABA}_{\mathrm{B}}$ receptors are present in the IC, which is supported by receptor binding (Chu et al., 1990; Milbrandt et al., 
1994; Fubara et al., 1996), in vitro studies (Zhang and Wu, 2000; Ma et al., 2002), and in vivo electrophysiological studies (Faingold et al., 1991; Vaughn et al., 1996). Activation of GABA $_{B}$ could influence acoustic responses in the ICC. Baclofen applied iontophoretically or systemically reduced sound-evoked responses, while phaclofen or CGP35348, the $\mathrm{GABA}_{\mathrm{B}}$ receptors antagonists, increased the resopnses to tone and sinusoidally amplitude modulated sounds (Faingold et al., 1991; Szczepaniak and Møller, 1996; Vaughn et al., 1996; Burger and Pollak, 1998). In addition, the GABA $A_{B}$ receptor plays an essential role in synaptic plasticity. Zhang and Wu (2000) showed that bath application of CGP35348 or phaclofen blocked the induction of long-term potentiation (LTP) and baclofen facilitated it. Collectively, GABAB receptors do have some modulation effects in the IC, but the nature of their functional role including pre- and postsynaptic $\mathrm{GABA}_{\mathrm{B}}$ receptors remains unclear.

\subsection{Purpose of the study}

Based on the studies reviewed above, we have known that the IC is a major structure in the central auditory system and plays a critical role in processing inputs from both the ascending and descending pathways (Winer, 2005). However, most of the studies focus on the ICC which has become most appreciated as a site for integration and relay of information along with auditory pathways. By contrast, little emphasis has been placed on investigating the properties of neurons in other subdivisions of the IC (Smith, 1992; Li et al., 1998, 1999; Syka et al., 2000). The functional role of the ICD in hearing is unknown (Winer and Schreiner, 2005). The present study aims to study intrinsic membrane properties and synaptic response properties of ICD neurons, along with 
investigation of the contribution of $\mathrm{GABA}_{B}$ receptors to the membrane excitability and synaptic transmission of ICD neurons. Results from this study will contribute to a better understanding of physiological properties of ICD neurons and the modulatory role of $\mathrm{GABA}_{\mathrm{B}}$ receptor in auditory information transmission.

\subsubsection{Intrinsic membrane properties of ICD neurons}

The ability of an ICD neuron to communicate with other neurons will be partly determined by its physiological state at the time it receives synaptic inputs. This physiological state will be shaped by its firing pattern and properties of the ionic currents that underlie its discharge. In the ICC, six distinct firing patterns have been uncovered. Each of these firing patterns was generated by distinct potassium currents (Sivaramakrishnan and Oliver, 2001). Despite the pivotal position of the ICD in the central auditory pathway and potential role the ICD plays in auditory processing, there are so far few physiological studies on the ICD, in contrast with the ICC for which much more extensive studies have been conducted. Only two studies using intracellular recording techniques examined intrinsic membrane and firing properties of IC cortex neurons including cells in ICD (Smith, 1992; Li et al., 1998). In these studies the sample of neurons was limited and the firing behavior was examined with injection of depolarizing current only. In Smith's study the data about ICD neurons were not distinguished from those of ICX neurons. These two studies showed similar results that ICD neurons had obvious $\mathrm{Ca}^{2+}$ potentials in response to deploarizing current injection and rebound depolarization upon the release of the hyperpolarization. However, there is no further information about how $\mathrm{Ca}^{2+}$ current in ICD neurons contributes to the 
membrane excitability. Therefore, the purpose of this study was to investigate intrinsic membrane properties of ICD neurons, including their passive membrane properties and active firing behavior, e.g., current-voltage relationship, firing pattern, rate-level function and characteristics of action potentials (APs). I then further investigated whether the firing behavior and membrane excitability of a neuron can be altered by a preceding membrane hyperpolarization and how calcium ions are involved in regulating membrane excitability in ICD neurons.

\subsubsection{Contribution of $G A B A_{B}$ receptors to membrane excitability and synaptic transmission of ICD neurons}

The presence of $\mathrm{GABA}_{\mathrm{B}}$ receptor within the $\mathrm{IC}$ has been demonstrated by quantitative receptor autoradiography and in vivo electrophysiological studies (Milbrandt et al., 1994; Fubara et al., 1996; Szczepaniak and Møller, 1996; Vaughn et al., 1996). Moreover, $\mathrm{GABA}_{\mathrm{B}}$ receptors are most prominent in the dorsal region of the $\mathrm{IC}$. GABA $\mathrm{B}$ receptors can express on both presynaptic terminals and postsynaptic membranes. In general, presynaptic $\mathrm{GABA}_{\mathrm{B}}$ receptors contribute to presynaptic inhibition by depressing the activation of $\mathrm{N}$ - and/or $\mathrm{P} / \mathrm{Q}$ type voltage-gated $\mathrm{Ca}^{2+}$ channels that directly facilitate $\mathrm{Ca}^{2+}$ influx for release of neurotransmitters (Bonanno et al., 1998; Takahashi et al., 1998;

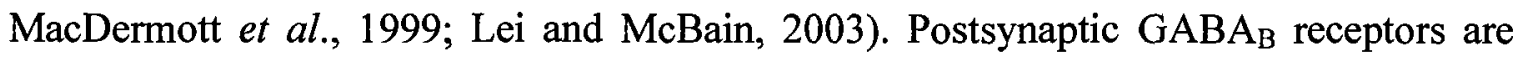
known to be coupled with $\mathrm{K}^{+}$channels mediating a slow inhibitory postsynaptic potential (Bowery, 1993; Kumamoto, 1997). In recent studies with an ICC slice preparation, activation of the $\mathrm{GABA}_{\mathrm{B}}$ receptors by baclofen induced supression of glutamatergic excitation and $\mathrm{GABA}_{\mathrm{A}}$ receptor-mediated inhibition. These effects may be a result of reduction of glutamate and $\mathrm{GABA}$ release through presynaptic $\mathrm{GABA}_{\mathrm{B}}$ receptors $(\mathrm{Ma}$ et 
al., 2002; Sun et al., 2006). But these studies did not find evidence of functional postsynaptic $\mathrm{GABA}_{\mathrm{B}}$ receptors in ICC neurons. Thus, the question arises as to whether GABA $_{B}$ receptor can modify membrane excitability and neurotransmission of ICD neurons. In other words, what are physiological properties of pre- and postsynaptic $\mathrm{GABA}_{\mathrm{B}}$ receptor in the ICD? The aim of my experiments, therefore, was to examine whether postsynaptic $\mathrm{GABA}_{\mathrm{B}}$ receptors modulate the excitability of ICD neurons through modulating different ionic currents and whether presynaptic $\mathrm{GABA}_{\mathrm{B}}$ receptor can inhibit the neurotransmission in ICD neurons. 

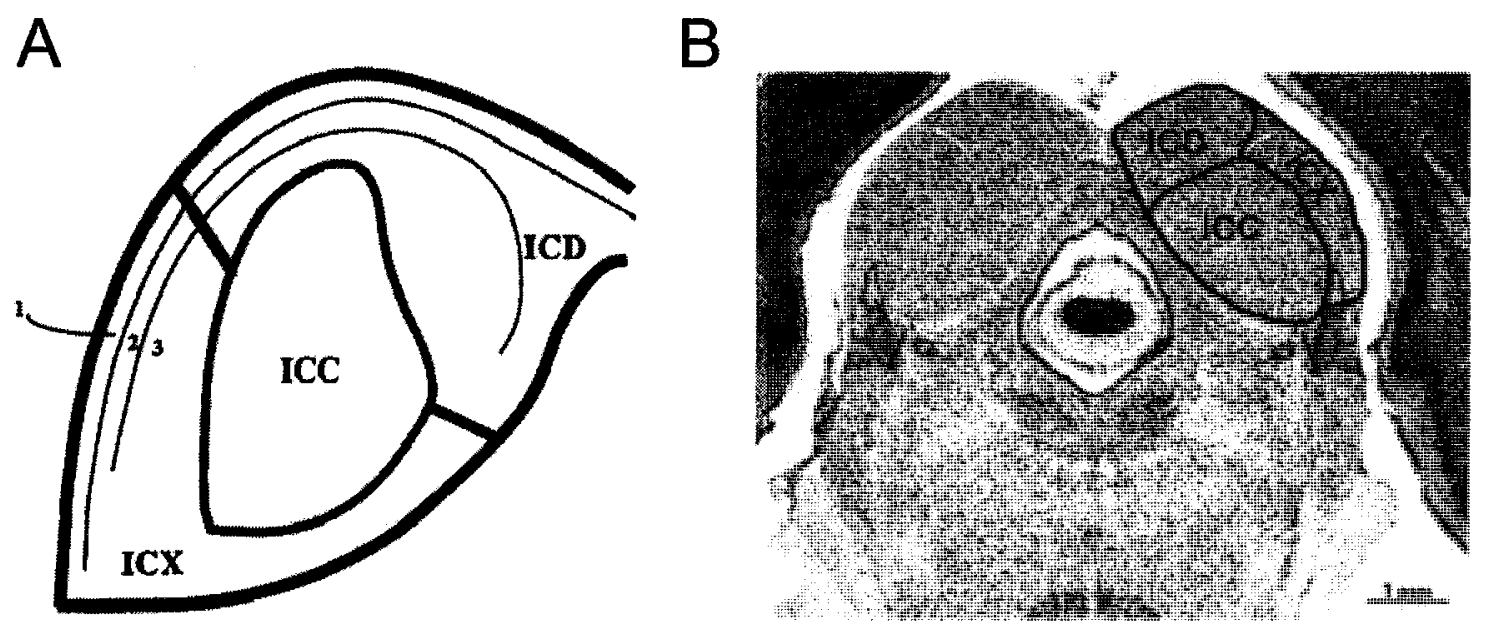

Figure 1.1. Subnuclei of the Inferior colliclus. A: Schematic diagram of the coronal section through the IC. It shows the central nucleus (ICC), the laterally and rostrally placed external cortex (ICX), and the dorsal cortex (ICD). Lines near the dorsal and lateral surfaces indicate a cortical cytoarchitecture (Layer 1,2 and 3) (Adapted from Huffman and Henson, 1990). B: Photomicrograph of a Nissl-Stained frontal section through the midbrain, showing the three subdivisions of the IC. (Adapted from Winer and Larue, 2001). 


\section{Chapter 2}

\section{Intrinsic membrane properties of neurons in the rat's dorsal cortex of the inferior colliculus: effects of pre-hyperpolarization on membrane excitability}

\subsection{Introduction}

Many in vivo electrophysiological studies have indicated that synaptic inhibition plays an important role in determining the output of auditory neurons (Covey and Casseday, 1999; Casseday et al., 2000; Galazyuk et al., 2005; Ingham and McAlpine, 2005; Nataraj and Wenstrup, 2005, 2006; Nayagam et al., 2005; Fuzessery et al., 2006; $\mathrm{Wu}$ and Jen, 2006). Inhibition regulates the firing rate, shapes the tuning curves, ratelevel functions and firing patterns of IC neurons in response not only to simple tone bursts but also to complex sounds, communication sounds or binaural signals. Timing of inhibition is also very important for encoding specific attributes of sounds (Faure et al., 2003; Paolini et al., 2004; Galazyuk et al., 2005; Nayagam et al., 2005). For example, an excitatory rebound that emerges after an inhibition can summate with a late excitatory response to enhance the neuron's membrane excitation and increase the likelihood of its firing. The interaction of excitation and inhibition can be a neuronal mechanism for encoding of specific characteristics of sounds (Casseday et al., 1994; Ehrlich et al., 1997; Covey and Casseday, 1999; Brand et al., 2000; Faure et al., 2003; Galazyuk et al., 2005). Evidence of the presence of early (or preceding) inhibition has been provided by intracellular and extracellular recordings from IC neurons in response to tones, noise and frequency-modulated (FM) sounds (Covey et al., 1996; Kuwada et al., 1997; Casseday et al., 2000; Galazyuk et al., 2005; Nayagam et al., 2005; Fuzessery et al., 2006; Nataraj and Wenstrup, 2005, 2006). Several models have been developed to explain neuronal 
coding of rate or direction of frequency-modulated tones, sound duration and harmonically complex sounds based on the assumption of summation or cancellation of synaptic inputs in particular temporal sequences, including inhibition preceding excitation.

To understand fully whether membrane excitability and firing behavior of an ICD neuron can be altered by preceding membrane hyperpolarization, the intrinsic membrane properties were examined first. Then I focused my examination on responses to a sequence of hyperpolarizing and depolarizing current injections in ICD neurons. I examined the firing rate, firing pattern, first spike latency and rate-level function of ICD neurons in response to depolarizing current injections following membrane hyperpolarization. My results show that membrane excitability and firing behavior of the majority of the ICD neurons could be altered by preceding hyperpolarization. ICD neurons that exhibited different firing patterns as identified by their responses to depolarizing current injection were qualitatively different in their reaction to prior membrane hyperpolarization. These results suggest that separate classes of ICD neurons process excitatory inputs following inhibitory inputs in different ways. Thus ICD neurons can provide various mechanisms for selective processing of specific auditory information.

\subsection{Materials and Methods}

\subsubsection{Preparation of brain slices}

In this study Wistar albino rats (Charles River, St. Constant, Quebec, Canada) 10 to 19 days old were used. All procedures involving animals were performed in compliance with the guidelines of the Canadian Council on Animal Care and were 
approved by the Carleton University Animal Care Committee. The experimental procedures were similar to those previously described by $\mathrm{Wu}$ et al. (2004) and Sun et al. (2006). Briefly, the animals were anesthetized with isoflurane and rapidly decapitated. The brain was removed and dissected in $24-26^{\circ} \mathrm{C}$ artificial cerebral spinal fluid (ACSF) containing (in mM): $129 \mathrm{NaCl}, 3 \mathrm{KCl}, 1.2 \mathrm{KH}_{2} \mathrm{PO}_{4}, 2.4 \mathrm{CaCl}_{2}, 1.3 \mathrm{MgSO}_{4}, 20 \mathrm{NaHCO}_{3}$, 3 HEPES, and 10 glucose at a pH of 7.4 and an osmolarity of $290-310 \mathrm{mOsm} / \mathrm{kg}$. The ACSF was continuously bubbled with $95 \% \mathrm{O}_{2}$ and $5 \% \mathrm{CO}_{2}$. Brain slices were then cut at $180-220 \mu \mathrm{m}$ in the frontal plane through the auditory midbrain with a tissue slicer. The slices containing ICD were stored in the oxygenated ACSF at room temperature for at least $1 \mathrm{hr}$ prior to any physiological recordings. The slice was then transferred to a recording chamber and completely submerged in the oxygenated ACSF, which circulated through the chamber at a flow rate of $6-8 \mathrm{ml} / \mathrm{min}$ with a temperature of $30-32^{\circ} \mathrm{C}$. The drug, ZD7288 (Tocris, Ellisville, MO. USA), was dissolved in ACSF and applied to the bath.

\subsubsection{Whole cell patch-clamp recording}

Electrodes for whole-cell patch clamp recording were prepared from thin-walled glass pipettes (Kimax-51; Kimble, Vineland, NJ) with a two-stage vertical puller (PP830, Narishige, Tokyo, Japan). The electrodes were filled with an internal solution containing (in mM): $130 \mathrm{~K}$-gluconate, $0.6 \mathrm{EGTA}, 10 \mathrm{HEPES}, 2 \mathrm{MgCl}_{2}, 5 \mathrm{KCl}, 2 \mathrm{ATP}, 0.3 \mathrm{GTP}$. The $\mathrm{pH}$ of the solution was adjusted to 7.25 with $\mathrm{KOH}$ and the osmolarity was $280-290$ $\mathrm{mOsm} / \mathrm{kg}$. The resistance of the patch electrode was 4-7 M $\Omega$.

With an EPC-8 patch-clamp amplifier (HEKA, Lambrecht, Germany), whole-cell patch clamp recordings were made from neurons located in the most dorsomedial region 
of the IC, an area which is considered to be well within the ICD (Oliver, 2005). ICD neurons were visualized under a Zeiss Axioskop microscope by a $40 \mathrm{X}$ water immersion objective with Hoffman modification contrast optics. The membrane potentials reported in this study were corrected using the junction potential (Neher, 1992). Series resistances were between 15-25 M $\Omega$ and were compensated by $50-70 \%$. If the series resistance changed by more than $15 \%$ of the initial value during the recording, the data were discarded. All the recordings were made under current clamp mode and bridge balance was applied. To examine the membrane excitability and firing behavior of ICD neurons, we applied depolarizing, hyperpolarizing, or a sequence of hyperpolarizing and depolarizing current pulses. Signals were filtered at $5 \mathrm{kHz}$, digitized at $10 \mathrm{kHz}$ by a Digidata 1200 interface, acquired by pClamp6, and analyzed off-line by pClamp8 (Axon Instruments, Union City, CA, USA).

\subsubsection{Data analysis}

Assessment of intrinsic membrane properties was made from the recordings at least 6-8 min after establishing a whole-cell configuration and acquiring a stable resting potential. Neurons with resting potentials more negative than $-55 \mathrm{mV}$ were accepted and used for further analysis. The intrinsic firing pattern of a neuron was determined by responses to a range of positive current injection from 0 to $300 \mathrm{pA}(10 \mathrm{pA} / \mathrm{step})$ for 200 ms. The spike latency was measured between the onset of current injection to the beginning of the spike. The total firing rate was calculated from the number of spikes occurring over the entire period $(200 \mathrm{~ms})$ of current injection. The active firing rate was calculated from the number of spikes over the period of the spike train. The spike 
accommodation was quantified by an adaptation ratio (AR), which was defined as the ratio of the last inter-spike interval to the first one in a train of 4-6 spikes. The properties of APs including spike amplitude and half-width were determined from recordings in which only one or two APs were elicited by depolarizing current injection. Spike amplitude was measured from the point at which the membrane potential started to rise rapidly to the value at the peak of the AP. The spike half-width was measured at $1 / 2$ amplitude of the AP. The AP threshold was defined as the membrane potential at which the neuron generated only one or two spikes. The changes in voltage elicited via negative current injection were measured between the resting membrane potential and the peak membrane hyperpolarization. These values were then used to plot the current-voltage (IV) curve. The slope of the IV curve that was derived from voltage changes elicited by 200 ms current injections from 0 to $-80 \mathrm{pA}$ was essentially linear and represented input resistance (M $\Omega$ ) of a cell membrane. The time constant $(\tau)$ of the cell membrane was determined by measuring the time required for the membrane potential to move $63 \%$ of the way towards its final value in response to a $-100 \mathrm{pA}$ current pulse. Numerical averages are presented as mean $₫$ SEMs. Statistical significance was evaluated by a paired Student's $t$ test or a one-way ANOVA with a post hoc Bonferroni test, and the minimum criterion for statistical significance was set at $p<0.05$.

\subsection{Results}

In total, 108 cells were recorded from ICD in 60 brain slices. The analysis was focused on data from 96/108 neurons $(88.9 \%$ ), which represented 3 major physiological cell types based on their firing patterns (Fig 2.1). These neurons showed regular, adapting 
or buildup firing patterns according to their responses to depolarizing current injection. The remaining 12 neurons (11.1\%) showed 3 other firing patterns (onset, transient and fast-adapting, Fig 2.2), but their responses were not analyzed further in this study. The resting membrane potentials of the 96 neurons were in a range of -56 to $-64 \mathrm{mV}$ (mean: $60.12 \pm 0.16 \mathrm{mV}$ ) throughout the recording period. Spontaneous spiking activity was very rare in these cells.

\subsubsection{Responses to injection of depolarizing currents}

Sustained pattern This pattern was the most common firing pattern in our sample, comprising 87 of $96(90.6 \%)$ cells. In some neurons of this group the response to subthreshold current injection showed a broad hump (see chapter 3), which may be mediated by $\mathrm{Ca}^{2+}$ current (Smith, 1992; Li et al., 1998). When a suprathreshold current was injected, the neurons fired continuously over the entire period of the current pulse without an obvious hump. According to the value of the adaptation ratio (AR, see Materials and Methods), neurons in this group could be further subdivided into two types: sustained-regular $(n=44 ; 44.9 \% ; A R<1.5)$ and sustained-adapting $(n=43 ; 43.9 \% ; A R \geq$ 1.5). The left panel in Figure 2.1A shows responses of an ICD neuron with a sustainedregular firing pattern. This cell responded with a train of spikes and relatively constant inter-spike intervals to suprathreshold current injections. The firing rates of the neuron increased progressively with increasing current strength (Fig. 2.1B). The first spike latencies decreased as the depolarizing current was increased (Fig. 2.1D). This reduction was less at higher current levels $(>150 \mathrm{pA})$ than at lower levels. The average AR of the sustained-regular firing neurons was $1.19 \pm 0.03$, ranging from 0.93 to 1.48 (Fig. 2.1E). 
The middle panel in Figure 1A shows an example of the sustained-adapting cells. Suprathreshold current produced a train of action potentials (APs), which occurred more frequently during the initial period of current injection. As a result, the inter-spike intervals of the later spikes were longer than those of the early spikes, and the AR was larger than that of the sustained-regular cells. The mean AR for this sub-group of neurons was 2.15 \pm 0.11 , ranging from 1.51 to 4.34 (Fig. 2.1E). The relationships of firing rates and first spike latencies to depolarizing current levels were similar for regular and adapting neurons (Fig. 2.1B and D).

Buildup pattern The buildup pattern comprised $9.4 \%(9 / 96)$ of the neurons in our sample. These neurons fired APs with a long delay, i.e., a long first spike latency (Fig 2.1A, right panel). During the delay the membrane potential gradually approached the threshold for eliciting action potential. As the current was increased the neurons produced more APs and the delays (i.e., first spike latencies) became progressively shorter (Fig. 2.1D). The first spike latencies of buildup neurons were much longer than those of regular and adapting neurons, and were still quite long $(\sim 40 \mathrm{~ms})$ even at high levels of current injection ( $>200 \mathrm{pA}$ ) (Fig. 2.1D). The average total firing rates of buildup neurons were lower than those of regular and adapting cells (Fig. 2.1B). However, the active firing rates (see Materials and Methods) of buildup neurons were similar to those of regular cells (Fig. 2.1C). These results indicate that for the buildup cells, although the generation of spikes was delayed, the neuron could fire at rates similar to those of regular cells. The AR of the buildup neurons was $0.85 \pm 0.03$, ranging from 0.72 to 0.96 (Fig. 2.1E). These values were typically less than 1 because the inter-spike intervals of the later spikes were shorter than those of the early spikes. 
The intrinsic membrane properties of the 3 types of neurons are summarized in Table 2.1. The resting potential, membrane input resistance and time constant, and AP amplitude and half-width were not significantly different for the 3 types of neurons ( $p>0.05$, one-way ANOVA). The AP threshold of the buildup neurons was more positive than that of the regular or adapting neurons $(\mathrm{p}<0.01$, Bonferroni post hoc test).

We also investigated whether intrinsic membrane properties of ICD neurons change with age over the developmental period around the onset of hearing. The purpose was to determine whether there were any developmental changes around hearing onset that might have influenced the outcome of our experiments. We compared the intrinsic membrane properties of regular and adapting neurons at 3 different periods around the onset of hearing, i.e., postnatal days 10-12 (P10-12), just before hearing onset, P13-15, immediately after hearing onset and P16-19, several days after hearing onset. We noticed that there were more regular than adapting cells at P10-12 and more adapting than regular cells at P16-19. Membrane properties of regular and adapting neurons did not have any significant change with age ( $p>0.05$, one-way ANOVA), except that the AP half-width of the adapting neurons at P16-19 was shorter than that at P10-12 ( $<<0.01$, Bonferroni post hoc test) (Table 2.2). The results indicate that during the period of hearing onset and the first week thereafter ICD neurons tend to have a shift of firing pattern from regular to adapting; however, their basic electrical properties do not change substantially. The buildup neurons were found in all of the three age groups $(\mathrm{P} 10-12, \mathrm{n}=3 ; \mathrm{P} 13-15, \mathrm{n}=2$; P16-19, $n=4)$. No statistical analysis of membrane properties of the buildup neurons was made because of the small number of the cells in each group. 


\subsubsection{Responses to injection of hyperpolarizing currents}

Neurons responded to negative current injection with membrane hyperpolarization, and the relationship between the current and voltage changes measured at the beginning of current injection was essentially linear (Fig. 2.3A). A striking feature of responses to hyperpolarizing current in some ICD neurons was a rebound depolarization when the current injection was discontinued (Fig. 2.3A, arrow in the inset). When the rebound was large, an anode break AP (or several APs) could be generated on the rebound (Fig. 2.3A and B). Usually a large rebound and anode break AP were elicited when the hyperpolarizing current was larger than $-100 \mathrm{pA}$ lasting for at least $150 \mathrm{~ms}$. The rebound in these cells may be mediated by $\mathrm{Ca}^{2+}$ current as it has been demonstrated to be eliminated by removal of extracellular $\mathrm{Ca}^{2+}$ (Smith, 1992; Sivaramakrishnan and Oliver, 2001; Ahuja and Wu, 2007). The rebound was observed in both regular and adapting neurons, but not in buildup neurons.

A voltage sag during hyperpolarization was observed in almost $70 \%$ of the ICD neurons sampled in this study (Fig. $2.3 \mathrm{~A}$ and $\mathrm{B}_{1}$, left panels). The depolarizing sag brought the membrane potential closer to the resting potential. It has been suggested that the sag is caused by activation of H-current $\left(I_{h}\right)$ demonstrated in neurons of various brain regions including auditory brainstem (Fu et al., 1997; Bal and Ortel, 2000; Koch and Grothe, 2003). In order to investigate whether $I_{\mathrm{h}}$ plays a role in generation of the rebound in ICD neurons, 4 neurons that showed a depolarizing sag during hyperpolarization and a rebound after hyperpolarization were tested with the $I_{\mathrm{h}}$ blocker, ZD7288 $(100 \mu \mathrm{M})$.

Figure 2.3B shows results obtained from a typical neuron. The voltage difference between the peak and steady levels of hyperpolarization increased as the current level 
was made more negative (Fig. $2.3 \mathrm{~B}_{1}$, right panel, indicated by $\mathrm{a}$ ). This result indicates an increased $I_{\mathrm{h}}$. After application of ZD7288, the sag was no longer observed (Fig. 2.3B left panel) and the difference between the peak and steady levels of hyperpolarization was almost zero (Fig. 2.3B $\mathrm{B}_{2}$, right panel, indicated by $\mathbf{m}$ ), which implies that the $I_{\mathrm{h}}$ was completely blocked. But the neuron still produced a rebound and spiking, although the generation of the rebound and spiking was delayed (Fig. $2.3 \mathrm{~B}_{2}$, left panel). Blockade of $I_{\mathrm{h}}$ would also increase neuronal input resistance (Surges et al., 2004), therefore causing an additional slight membrane hyperpolarization (Fig. $2.3 \mathrm{C}$ ). To rule out the possibility that the generation of the rebound during ZD7288 was due to a larger hyperpolarization, we adjusted the current amount to a less negative value in order to generate the same steady level of hyperpolarization when the slice was in ACSF and in ZD7288 solution. In both conditions, the rebound and spiking were elicited by hyperpolarization (Fig. 2.3D). All these results suggest that in ICD neurons initiation of the rebound does not rely on activation of $I_{h}$.

\subsubsection{Responses to depolarizing currents following membrane hyperpolarization}

To investigate further how pre-hyperpolarization affects responses to subsequent depolarization, we applied a sequence of hyperpolarizing and depolarizing current to 88 neurons in our sample, which included 82 sustained-regular and adapting neurons and 6 buildup neurons. Among these 88 neurons, 45 were rebound cells and 43 were nonrebound cells. We used a protocol in which a hyperpolarizing current pulse of $-200 \mathrm{pA}$ for $250 \mathrm{~ms}$ was injected prior to a series of depolarizing current injections from 10 to $200 \mathrm{pA}$ (10 pA/step) for $300 \mathrm{~ms}$. We set the fixed duration and strength of pre-hyperpolarization 
in order to ensure generation of a sufficiently large rebound in all the tested neurons. Thus, effects of the rebound on subsequent firing could be assessed.

\section{Non-rebound neurons}

In some regular or adapting non-rebound cells $(19 / 37,51.4 \%)$, the membrane excitability and firing pattern were unchanged in response to depolarizing current following pre-hyperpolarizing current. Figure 2.4 shows an example. This neuron had regular firing in response to a depolarizing current (Fig. 2.4A, left panel). After prehyperpolarization the neuron still showed a regular firing pattern (Fig. 2.4A, right panel). We plotted latencies of each spike in the train for the two levels (60 pA and $200 \mathrm{pA})$ of depolarizing current with (open circles and triangles) and without (solid circles and triangles) pre-hyperpolarization as a function of AP order (Fig. 2.4B, right panel). The relationships between the spike latency and AP order were nearly linear and similar for the two conditions, indicating regular firing and also confirming that the firing pattern was unchanged after pre-hyperpolarization. We further analyzed and compared first spike latencies and firing rates when the neuron responded to depolarization alone or depolarization (for initial $200 \mathrm{~ms}$ ) following pre-hyperpolarization. Both first spike latencies and firing rates were very similar in these two conditions (Fig. 2.4B, left and middle panels).

However, the firing patterns of the remaining non-rebound sustained neurons were changed to pauser $(n=7)$ or buildup $(n=11)$ after pre-hyperpolarization. Figure 4 shows responses of a neuron whose firing was changed to a pauser pattern. Following pre-hyperpolarization, the neuron responded to depolarizing current (70 pA and $140 \mathrm{pA}$ ) with an onset spike followed by a long pause and then a train of spikes (Fig. 2.5A, right 
panel). The relationship between the latency of each spike and AP order was changed from linear to non-linear with a longer latency of the $2^{\text {nd }}$ spike, indicating that the firing pattern was changed from regular to pauser (Fig. 2.5B, right panel). However, the latencies of the onset spikes, i.e., first spike latencies, became shorter compared to those in response to depolarization alone (Fig. 2.5B, left panel). The difference was more evident at lower than higher levels of current. As expected, the firing rate was decreased after pre-hyperpolarization as compared to the responses to depolarization alone (Fig. 2.5B, middle panel). Figure 2.6A shows a representative neuron whose firing pattern was changed from regular to buildup. The first spike latencies were much longer and the firing rate was greatly decreased compared to responses to depolarization alone (Fig. $2.6 \mathrm{C}$ ). In 2 out of the 11 cells, the firing pattern was changed to buildup after prehyperpolarization, but the buildup pattern then turned to pauser as depolarizing current was further increased.

For each of the original 6 buildup neurons tested, pre-hyperpolarization did not alter the firing pattern, but led to an increase in first spike latency and a decrease in firing rate. Figure $2.6 \mathrm{~B}$ and $\mathrm{D}$ show these changes in a typical example of this group of neurons. Rebound Neurons

The rebound neurons are expected to exhibit an enhancement of the membrane excitability during the rebound. To test this hypothesis, we analyzed the firing behavior of all the 45 rebound neurons in response to injections of a fixed level of negative current followed by a series of positive current pulses.

As expected, the firing rate was increased in some cells (13 of 45 cells, $28.9 \%$ ). A typical example is shown in Figure 2.7. Positive current injection led to a train of spikes 
with a regular pattern (Fig. 2.7A, left panel). A low level (-50 pA) of negative current injection induced a membrane hyperpolarization that was followed by a small rebound and a spike (Fig. 2.7A, upper trace in middle panel). A higher level (-150 pA) of negative current injection produced a larger hyperpolarization and a large rebound depolarization from which several APs arose (Fig. 2.7A, bottom trace in middle panel). In response to depolarizing current injection following pre-hyperpolarization, the firing behavior of this neuron was altered (Fig. 2.7A, right panel). The regular firing was changed to adapting. The relationships of spike latencies against AP order were changed from linear to nonlinear with longer latencies for the later spikes, indicating a change of the firing pattern from regular to adapting (Fig. 2.7B, right panel). For the original adapting neurons, the adaptation became more pronounced after pre-hyperpolarization. The firing rates were greatly increased for the initial $200 \mathrm{~ms}$ current injection after pre-hyperpolarization compared to those without pre-hyperpolarization (Fig. 2.7B, middle panel). All of these results suggest that the neuron's membrane excitability was enhanced after prehyperpolarization.

In contrast to the neurons whose membrane excitability was enhanced following pre-hyperpolarization, some cells (19 of 45 cells, 42.2\%) showed suppression of membrane excitability after pre-hyperpolarization. There were two groups of neurons in this category, showing slightly different firing behavior. In the first group, the neurons fired with a pauser pattern after pre-hyperpolarization. Figure $2.8 \mathrm{~A}$ shows an example. A depolarization after pre-hyperpolarization elicited 2 onset spikes followed by a long pause and a subsequent train of spikes (Fig. 2.8A, right panel). The firing rate of this neuron was decreased especially at higher levels of depolarizing current (Fig. 2.8B, lower 
panel). In the second group, the firing pattern was changed to onset after prehyperpolarization (Fig. 2.8C, right panel). Thus, the firing rates were greatly reduced (Fig. $2.8 \mathrm{D}$, lower panel).

In remaining neurons (13 of 45 cells, $28.9 \%$ ) pre-hyperpolarization had no effect on firing rates, however, the firing pattern became irregular. Figure 8 shows an example of this type of response. This cell responded to positive current injections with a regular firing pattern (Fig. 2.9A, left panel). Hyperpolarizing current injection led to membrane hyperpolarization followed by a rebound depolarization and spikes (Fig. 2.9A, middle panel). When the neuron was tested again with the same levels of positive current as shown in the left panel, but following hyperpolarizing current injection, the neuron showed irregular firing (Fig. 2.9A, right panel). After pre-hyperpolarization the relationships between latencies and order of spikes became somewhat non-linear and irregular (Fig. 2.9B, right panel). The firing rate remained about the same (Fig. 2.9, middle panel), however, the distribution of spikes over the course of the response seemed to change considerably. Nevertheless, the membrane excitability of this group of neurons was little affected by pre-hyperpolarization.

In spite of three distinct changes in firing pattern and firing rate produced by prehyperpolarization in different rebound neurons, there were similar changes in the first spike latency and the relationship between the first spike latency and current level in all the rebound neurons (Fig. 2.7B, left panel; Fig. 2.8B, upper panel; Fig. 2.8D, upper panel; Fig. 2.9B, left panel). Without pre-hyperpolarization the first spike latency progressively decreased in response to increases in depolarizing current. With preceding hyperpolarization the first spike latency became shorter and remained relatively constant 
at all levels of depolarizing current injection.

In summary, various effects on membrane excitability and firing behavior by a preceding hyperpolarization were manifested in different ICD neurons. Figure 2.10 shows comparisons of first spike latencies and firing rates in response to depolarization alone or depolarization with a preceding hyperpolarization in all of the 88 neurons tested. The first spike latencies and firing rates were averaged from those of responses to current injections of 5 steps ( $20 \mathrm{pA} / \mathrm{step})$ above the threshold. The results showed that the first spike latencies and firing rates were affected differently in different neurons. Changes in first spike latency and firing rate were related to the change in firing pattern as well as the presence or absence of the rebound.

Of the non-rebound (NR) neurons, $44.2 \%$ did not show any change in their first spike latencies or firing rates, and their firing pattern remained sustained (NR-U group). However, $41.9 \%$ of these neurons showed a decrease in firing rate, and their firing pattern was changed from sustained to pauser (NR-P group, 16.3\%) or buildup (NR-B group, $25.6 \%$ ). In both groups, first spike latencies were altered, but in opposite directions. The first spike latencies were shortened for NR-P group, but lengthened for NR-B group. For the remaining $13.9 \%$ of the non-rebound neurons, their firing pattern was a buildup in response to depolarizing current injection alone, but this pattern was unchanged when the neurons were subjected to pre-hyperpolarization. This group was named as NRB-B group. For neurons in this group the firing rates were decreased and first spike latencies were greatly prolonged after pre-hyperpolarization. Overall, except for the NR-U group, the membrane excitability of all the non-rebound cells was suppressed after preceding hyperpolarization. 
For the rebound cells, the first spike latency in response to depolarizing current was significantly shorter with pre-hyperpolarization compared to that without prehyperpolarization. Pre-hyperpolarization also exerted various effects on firing behavior of the rebound neurons. Some rebound neurons showed a decrease in firing rate, and their firing pattern was changed from sustained to onset (R-O group, 20.0\%) or pauser (R-P group, $22.2 \%$ ). However, in other rebound neurons the firing rate was increased and the pattern was changed from regular to adapting or the adapting pattern became more evident (R-A group, 28.9\%). In the remaining rebound neurons, the firing rate was not altered but the firing pattern was changed from sustained-regular or -adapting to irregular (R-I group, 28.9\%). Overall, preceding hyperpolarization suppressed, enhanced or had little effect on membrane excitability of different rebound neurons. In summary, membrane excitability and/or firing behavior were modified by preceding membrane hyperpolarization in 69/88 (78.4\%) neurons. 


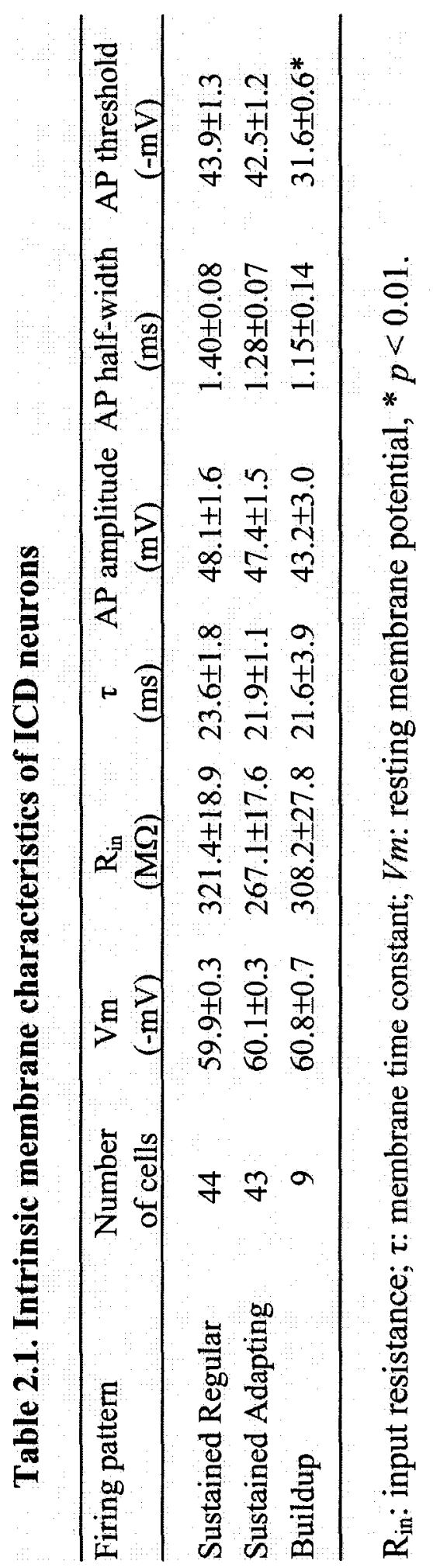




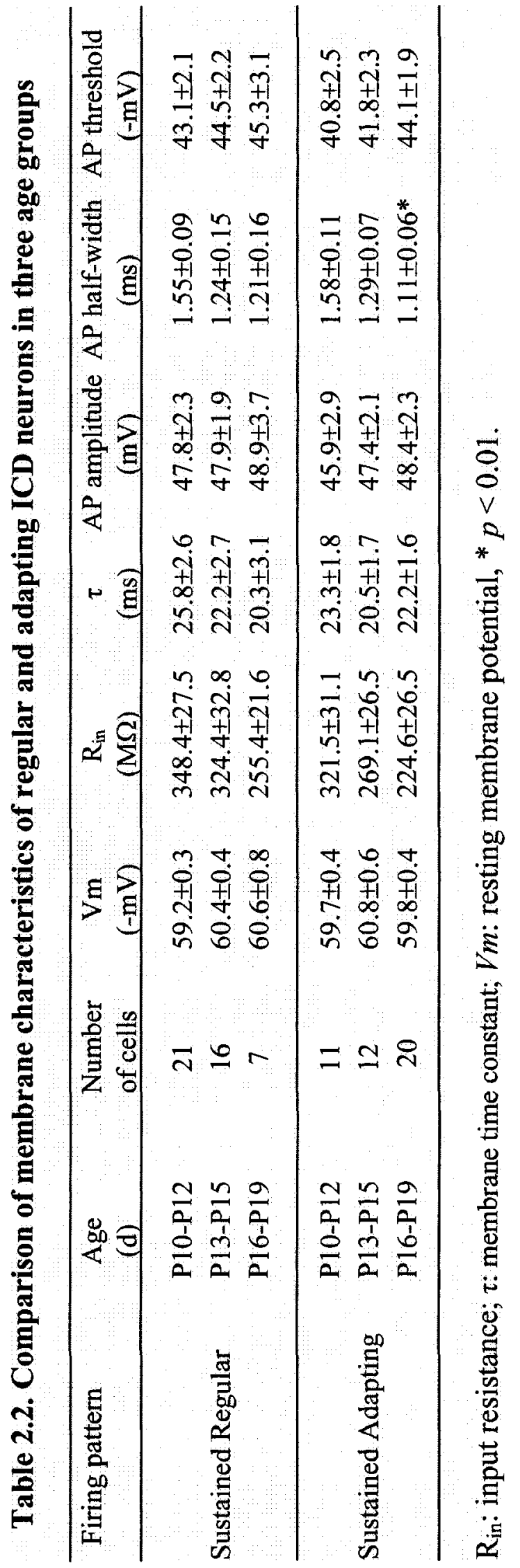




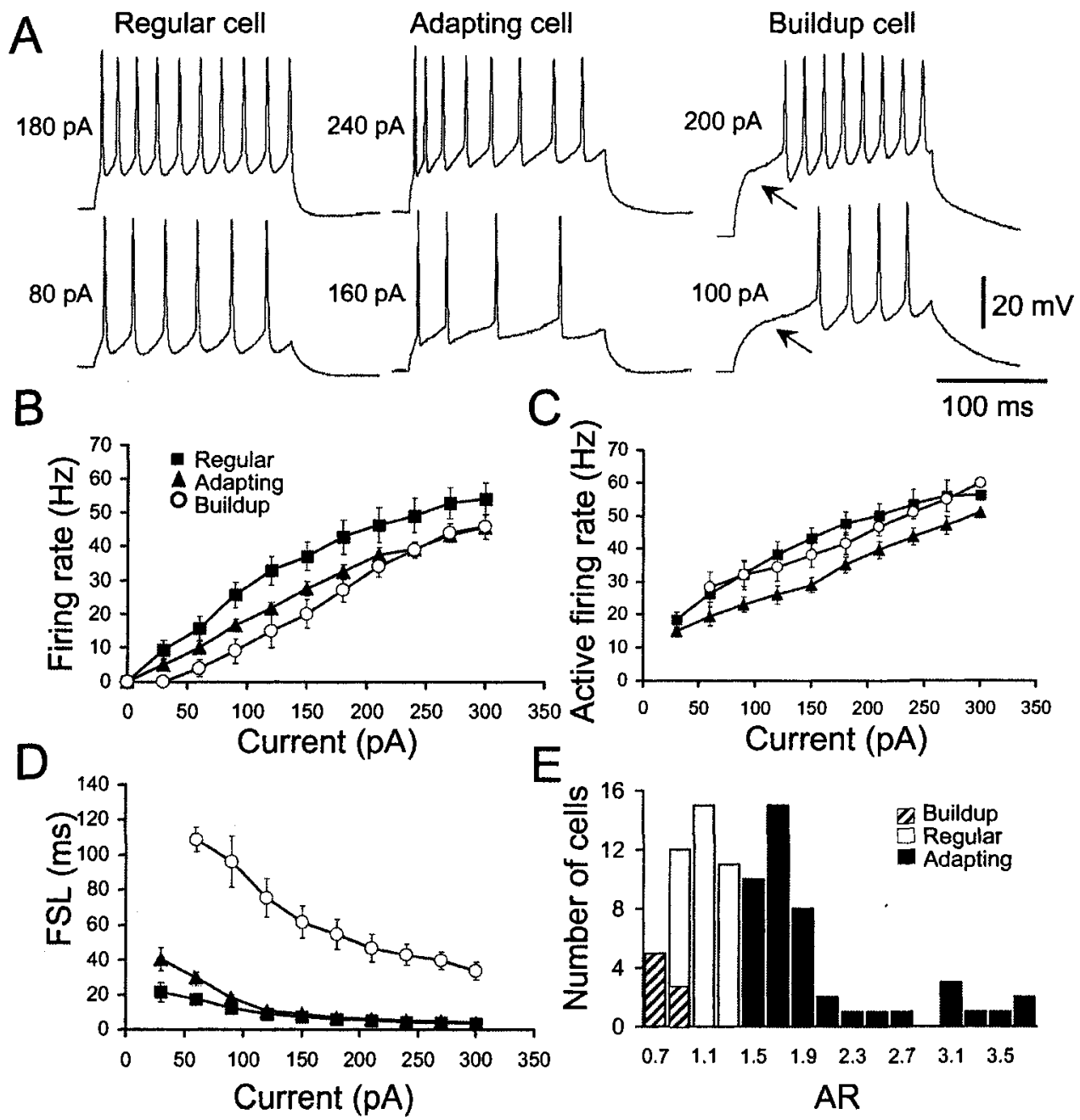

Figure 2.1. 


\section{Figure 2.1. Three major firing patterns classified according to responses to} depolarizing current injection. A. Left panel: Responses of a neuron with a regular firing pattern to a pulse of depolarizing current at two levels. The inter-spike intervals were relatively constant throughout the spike train $(A R=1.19)$. Middle panel: Responses of a neuron with an adapting firing pattern to a pulse of depolarizing current at two levels. The inter-spike intervals progressively increased over the spike train $(\mathrm{AR}=2.38)$. Right panel: Responses of a neuron with a buildup firing pattern to a pulse of depolarizing current at two levels. The firing started after a long delay. During the delay the membrane potential gradually built up (indicated by arrows) toward the threshold of action potential. The inter-spike intervals were relatively constant $(\mathrm{AR}=0.96)$ over the spike train. $\mathbf{B}$. The relationship of the firing rate to the current level over the entire period of current injection for three types of neurons. C. The relationship of the firing rate to the current level over the period of the spike train for three types of neurons. D. The relationship of the first spike latency (FSL) to the current level for three types of neurons. The data were obtained from 10 regular, 10 adapting and 6 buildup neurons. Vertical bars indicate standard errors of the means. E. The distribution of ARs across neurons with three distinct firing patterns $(n=96)$. 


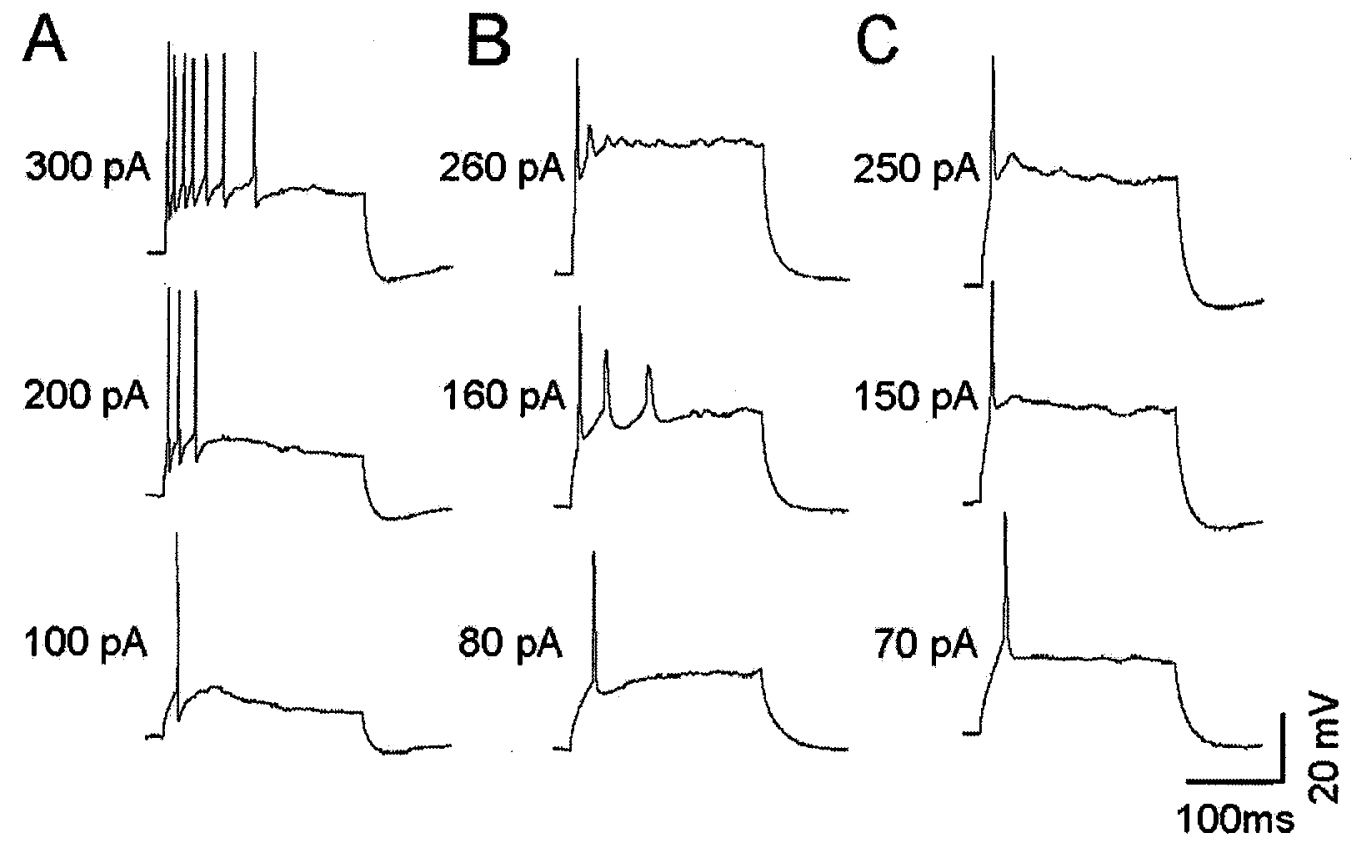

Figure 2.2. 
Figure 2.2. Other firing patterns of ICD neurons. A-C. Responses to depolarizaing current pulses of $200 \mathrm{~ms}$ at three levels indicated on the left side of each trace. A. Fast adapting pattern $(n=3)$. Note that action potentials were generated only at the initial period of membrane depolarization. B. Transient pattern $(n=5)$. Note that a full size action potential was followed by a few small spikes. C. Onset pattern $(n=4)$. Note that only one action potential was generated at the beginning of current injection. 
A
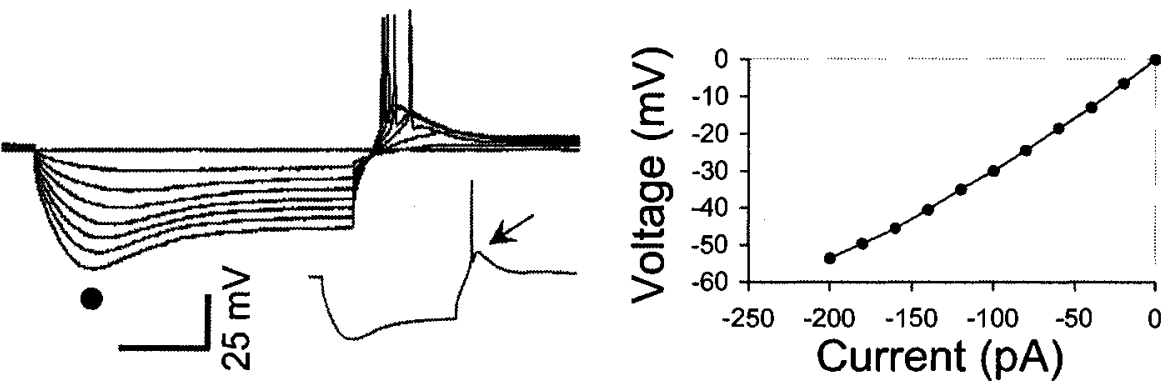

B
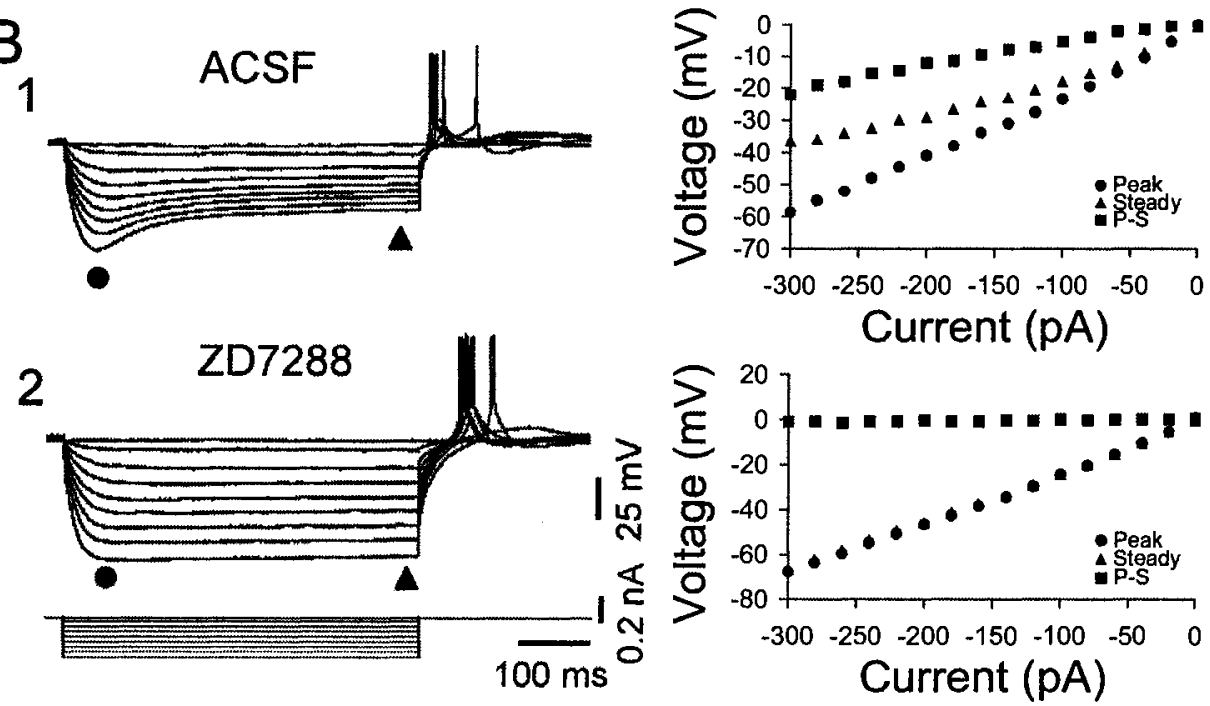

C

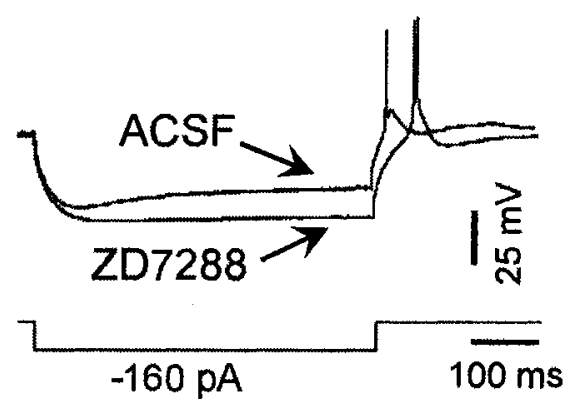

D

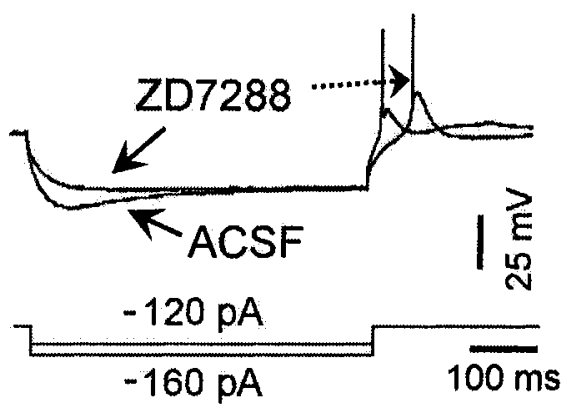

Figure 2.3. 
Figure 2.3. Responses to hyperpolarizing current injection in two neurons. A. Left panel: Responses to hyperpolarizing current injections of 7 levels in a neuron. A rebound depolarization and spiking were elicited after hyperpolarization. Inset: a response to a current pulse of $-80 \mathrm{pA}$. Time scle: $50 \mathrm{~ms}$. An arrow points to the rebound. Right panel: The relationship between the current level and voltage change measured at the peak hyperpolarization. $\mathbf{B}_{1}$. Left panel: Responses to hyperpolarizing current injections of 8 levels in a neuron. Note a depolarizing sag during the hyperpolarization, and a rebound and spiking following the hyperpolarization. Right panel: The relationships of the voltage change at the peak $(\bullet)$ and steady $(\boldsymbol{\Delta})$ hyperpolarization, and the voltage difference between the peak and steady hyperpolarization ( $\boldsymbol{(})$ to the current level. $\mathbf{B}_{2}$. Left panel: Responses of the same neuron to the same levels of hyperpolarizing current injections as shown in $2 \mathrm{~B}_{1}$ during application of ZD7288 $(100 \mu \mathrm{M})$. Note the absence of the depolarizing sag during the hyperpolarization and the persistence of the rebound and spiking after the hyperpolarization. Right panel: The relationships of the voltage change at the peak (•) and steady $(\boldsymbol{\Delta})$ hyperpolarization to the current level in ZD7288. The two curves are almost identical. The voltage differences between the peak and steady hyperpolarization ( $\square$ ) at all the levels of current injection were almost zero in ZD7288. C. Responses of this neuron to a current pulse of $-160 \mathrm{pA}$ in ACSF and ZD7288 solution. The rebound and spiking were generated in both conditions. D. Responses of this neuron to a pulse of $-160 \mathrm{pA}$ in ACSF and to a pulse of $-120 \mathrm{pA}$ in ZD7288. These two different levels of current produced the same level of the steady hyperpolarization. The rebound and spiking were still generated in both conditions. 

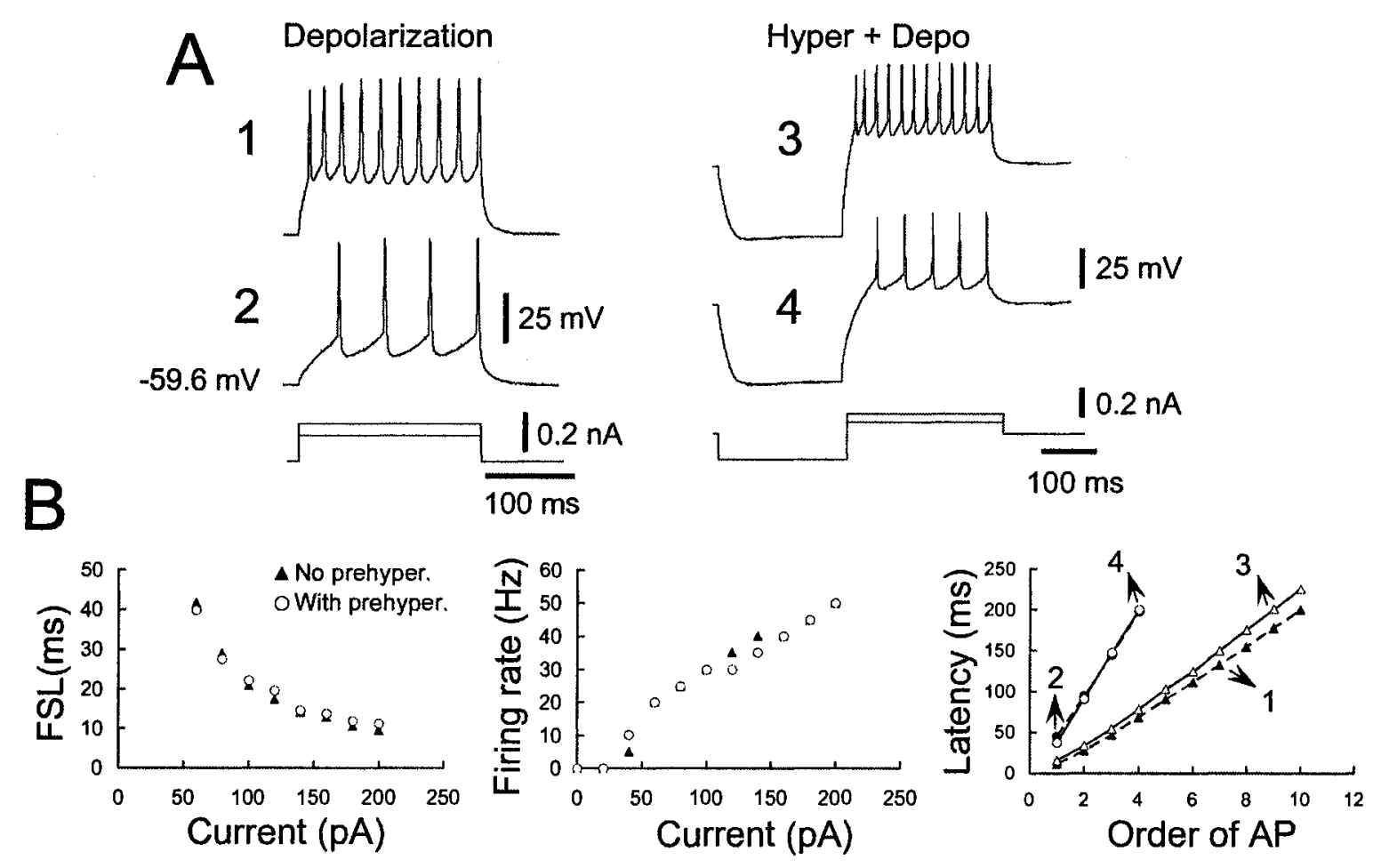

Figure 2.4. 
Figure 2.4. Pre-hyperpolarization did not affect the firing pattern in a sustainedregular neuron. A. Left panel: Responses to two depolarizing current pulses (top trace for $200 \mathrm{pA}$ and bottom trace for $60 \mathrm{pA}$ ). Right panel: Responses to a hyperpolarizing current pulse of $-200 \mathrm{pA}$ followed by the same levels of depolarizing current pulses as shown in the left panel. Note that the firing pattern remained regular while responding to positive current following a pre-hyperpolarization. B. Left and middle panels: The relationships of the first spike latency and firing rate to the magnitude of depolarizing current pulses with and without a pre-hyperpolarization. Right panel: The relationship between the latency of each spike and the spike order at the two levels of current injection without a pre-hyperpolarization (from traces 1 and 2 shown in $\mathbf{A}$ ) and with a pre-hyperpolarization (from traces 3 and 4 shown in A). Note that the first spike latencies, firing rates and latencies of all of the spikes were little altered by pre-hyperpolarization. 


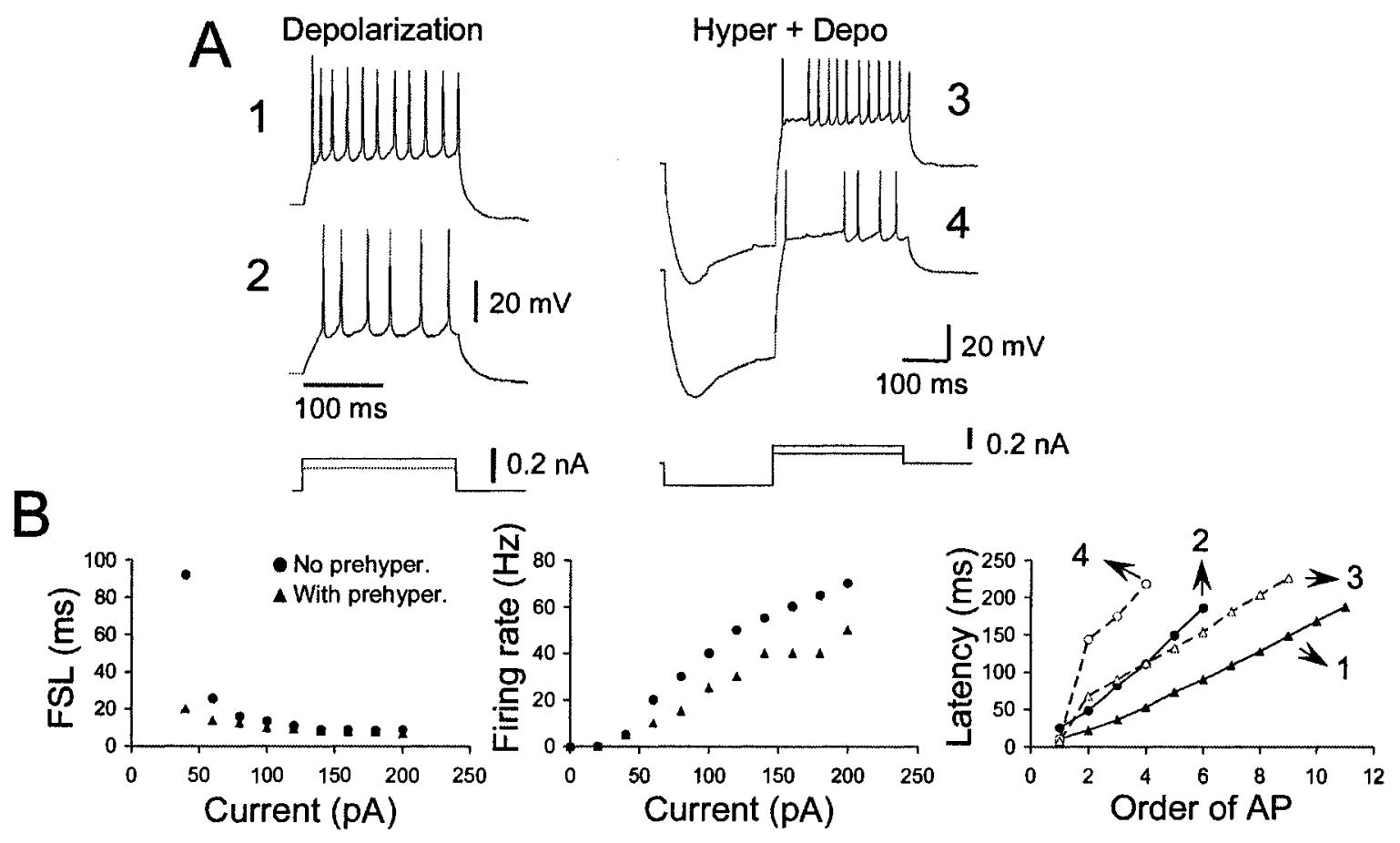

Figure 2.5. 
Figure 2.5. A sustained firing pattern was changed to a pauser pattern by prehyperpolarization in a neuron. A. Left panel: Responses to two depolarizing current pulses (top trace for $150 \mathrm{pA}$ and bottom trace for $50 \mathrm{pA}$ ), showing sustained firing. Right panel: after a hyperpolarizing current injection of $-200 \mathrm{pA}$, the neuron responded to subsequent depolarizing current injection at the same levels as shown in the left panel with a pauser firing pattern. B. Left and middle panels: The relationships of the first spike latency and firing rate to the magnitude of depolarizing current pulse with and without a pre-hyperpolarization. Right panel: The relationship between the latency of each spike and the order of spike at the two levels of current injection without a prehyperpolarization (from traces 1 and 2 shown in $\mathbf{A}$ ) and with a pre-hyperpolarization (from traces 3 and 4 shown in A). Note that the first spike latencies and firing rates were decreased, and the first inter-spike intervals were prolonged after pre-hyperpolarization compared to those in response to depolarizing current alone. 


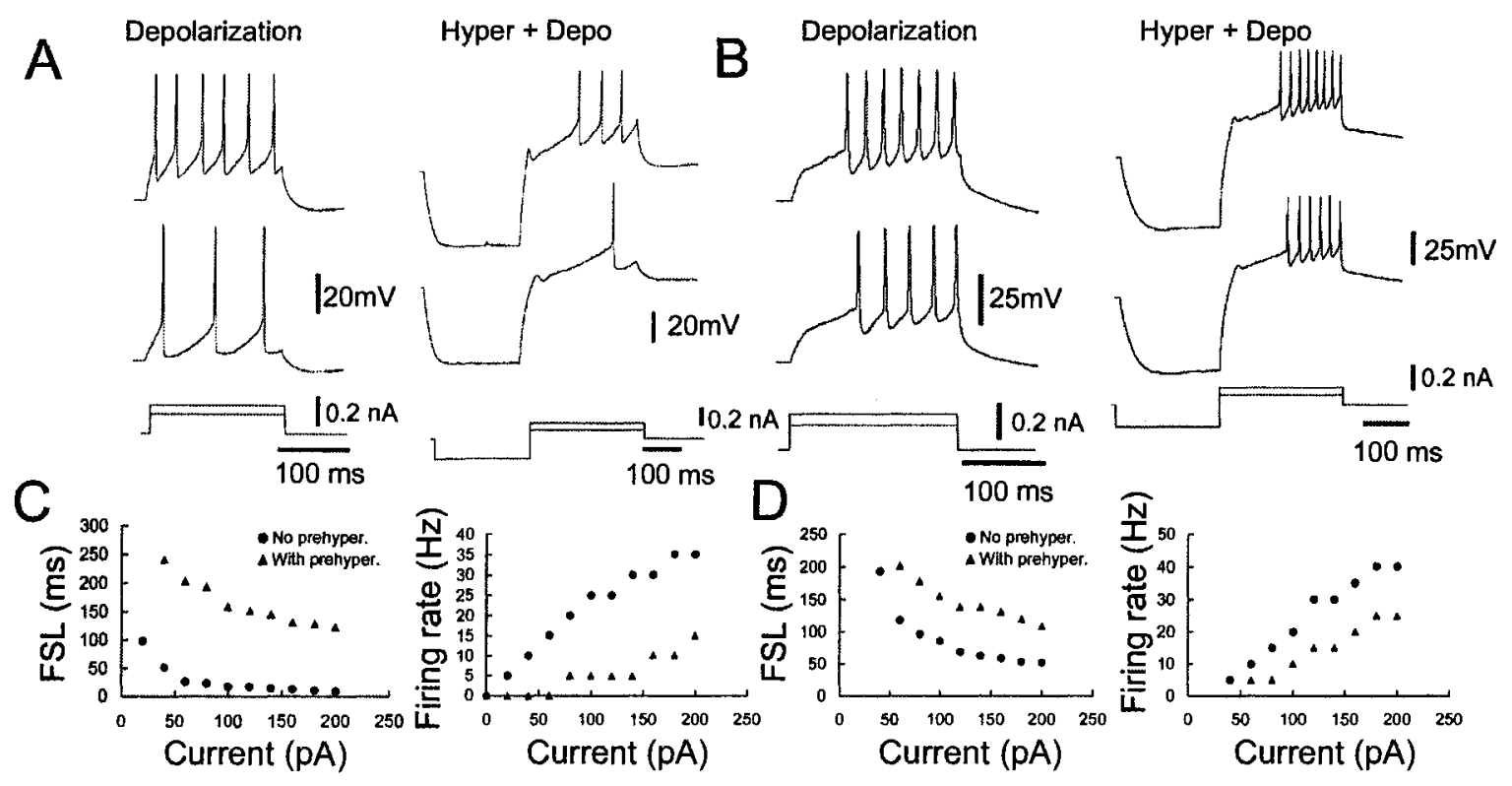

Figure 2.6. 
Figure 2.6. A firing pattern was changed from sustained to buildup or remained buildup after pre-hyperpolarization in two neurons. A. Left panel: Responses to depolarizing current injection at two levels (top trace: $160 \mathrm{pA}$ and bottom trace: $60 \mathrm{pA}$ ) in a neuron. Right panel: Responses to the same levels of depolarizing current as shown in the left panel following a hyperpolarizing current pulse of $-200 \mathrm{pA}$. Note that the firing pattern of this neuron was changed to buildup. B. Left panel: Responses to depolarizing current injection at two levels (top trace: $160 \mathrm{pA}$ and bottom trace: $110 \mathrm{pA}$ ) in another neuron, showing a buildup pattern. Right panel: Responses to the same levels of depolarizing current as shown in the left panel following a hyperpolarizing current pulse of $-200 \mathrm{pA}$. Note that the firing pattern remained buildup. C. The relationships of the first spike latency and firing rate to the current level with and without a pre-hyperpolarization for the neuron shown in A. D. The relationships of the first spike latency and firing rate to the current level with and without a pre-hyperpolarization for the neuron shown in $\mathbf{B}$. Note that both first spike latencies and firing rates were decreased with prehyperpolarization in the two neurons. 

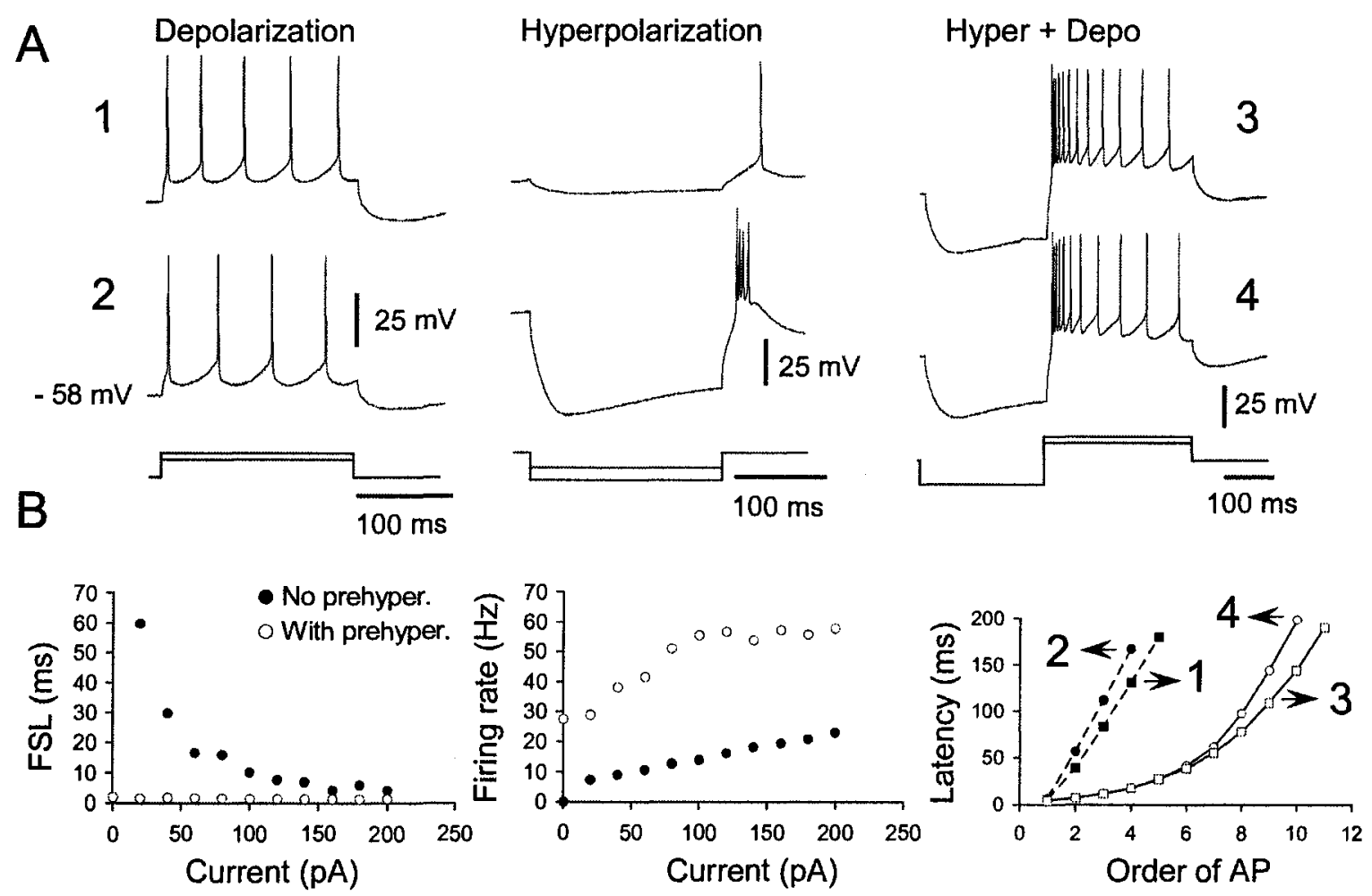

Figure 2.7. 
Figure 2.7. A regular firing pattern was changed to adapting and firing rates were increased by pre-hyperpolarization in a rebound neuron. A. Left panel: Responses to depolarizing current injection at two levels (top trace: $200 \mathrm{pA}$ and bottom trace: $150 \mathrm{pA}$ ), showing a regular firing pattern. Middle panel: Responses to two hyperpolarizing current pulses (top trace: $-50 \mathrm{pA}$ and bottom trace: $-150 \mathrm{pA}$ ). A rebound and one or several spikes were generated after hyperpolarization. Right panel: Responses to the same levels of depolarizing current as shown in the left panel following a hyperpolarizing current pulse of $-200 \mathrm{pA}$. Note more spikes at the early than the late period of the injection, indicating adapting firing. B. Left and middle panels: The relationships of the first spike latency and firing rate to the current level with and without a pre-hyperpolarization. Note that the first spike latencies were greatly decreased and the firing rates for the initial $200 \mathrm{~ms}$ of the depolarizing current pulse were substantially increased compared to those without prehyperpolarization. After pre-hyperpolarization the first spike latencies were constant as the level of depolarizing current injection was increased. Right panel: The relationship between the latency of each spike and the order of the spike without a prehyperpolarization (from traces 1 and 2 shown in A) and with a pre-hyperpolarization (from traces 3 and 4 shown in $\mathbf{A}$ ). The longer inter-spike intervals of the late spikes than those of the early ones indicate adapting firing. 

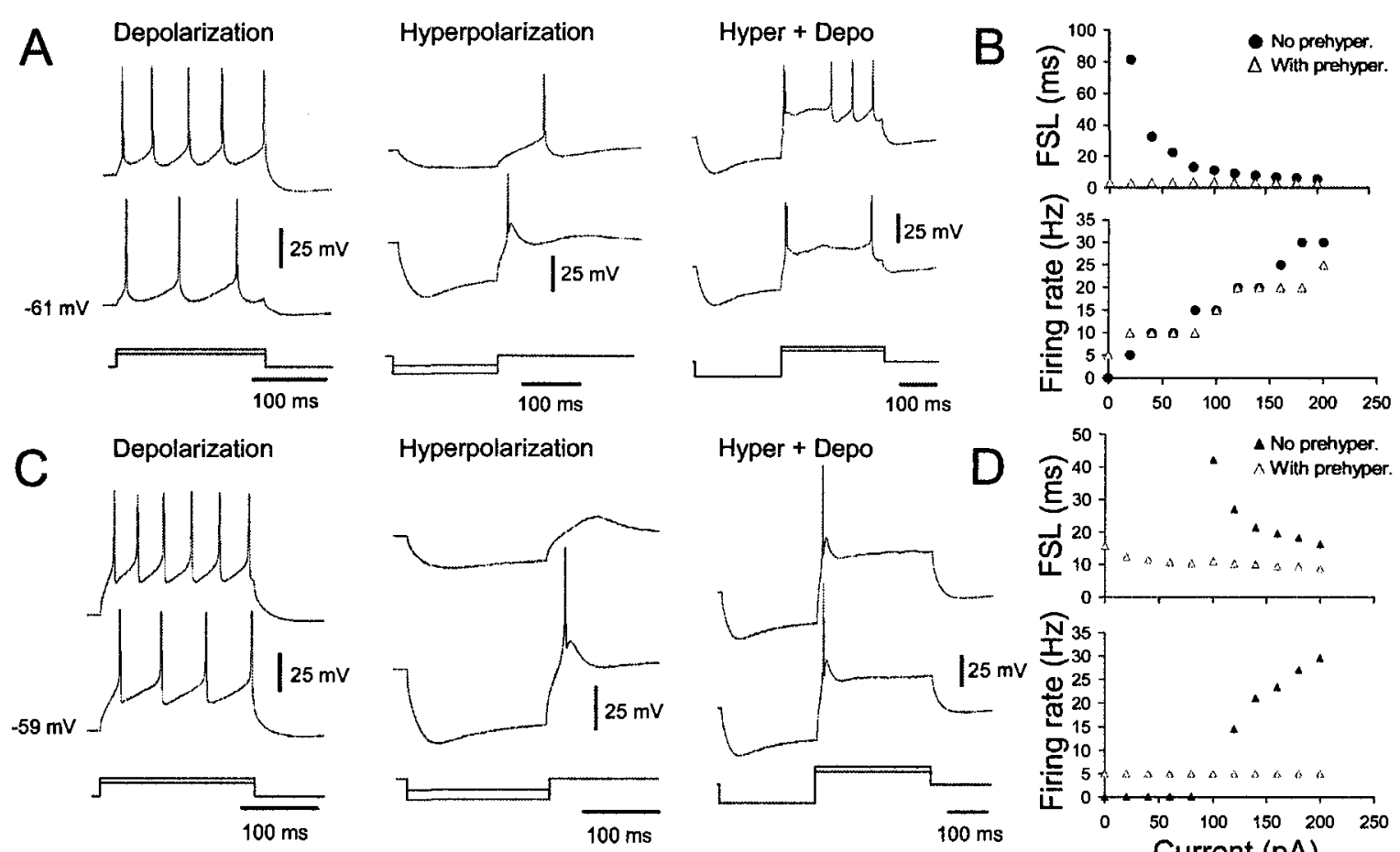

Hyperpolarization
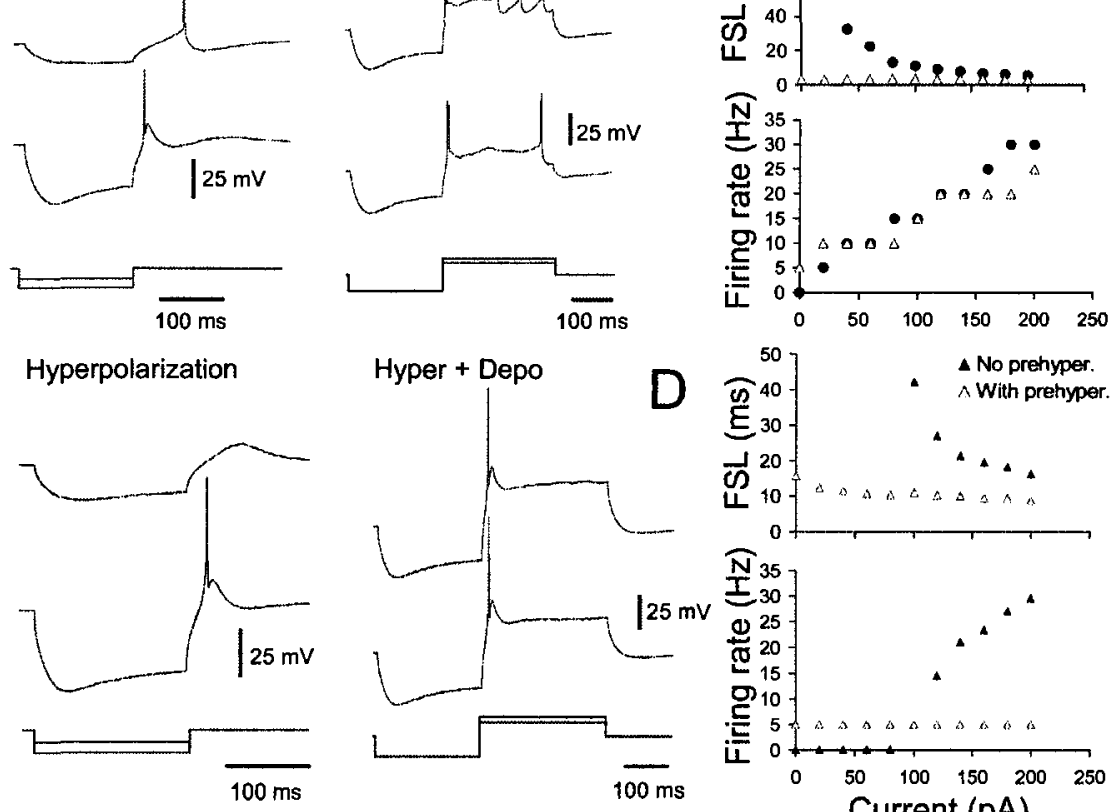

Ш $10-0 \Delta A \Delta A \Delta \Delta A$

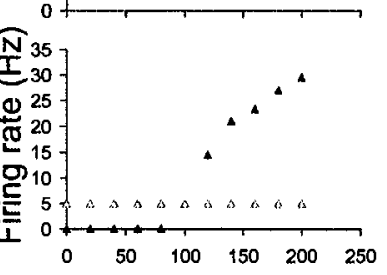

Current $(\mathrm{pA})$

Figure 2.8. 
Figure 2.8. A firing pattern was changed from sustained to pauser or onset, and firing rates were decreased by pre-hyperpolarization in two rebound neurons. $A$. Left panel: Responses of a neuron to depolarizing current injection at two levels (top trace: $200 \mathrm{pA}$ and bottom trace: $150 \mathrm{pA}$ ), showing a regular firing pattern. Middle panel: Responses of the same neuron to two hyperpolarizing current pulses (top trace: $-50 \mathrm{pA}$ and bottom trace: $-150 \mathrm{pA})$. A rebound and spikes were generated after hyperpolarization. Right panel: Responses to the same levels of depolarizing current as shown in the left panel following a hyperpolarizing current pulse of $-200 \mathrm{pA}$. Note a long pause after the initial 2 spikes during the subsequent depolarization. B. The relationships of the first spike latency and firing rate to the current level with and without a pre-hyperpolarization. C. Left panel: Responses of another neuron to depolarizing current injection at two levels (top trace: $200 \mathrm{pA}$ and bottom trace: $150 \mathrm{pA}$ ), showing a regular firing pattern. Middle panel: Responses to two hyperpolarizing current pulses (top trace: $-50 \mathrm{pA}$ and bottom trace: $-150 \mathrm{pA})$. A rebound and a spike were generated after hyperpolarization. Right panel: Responses to the same levels of depolarizing current as shown in the left panel following a hyperpolarizing current pulse of $-200 \mathrm{pA}$. Note an onset firing pattern after pre-hyperpolarization. D. The relationships of the first spike latency and firing rate to the current level with and without a pre-hyperpolarization. 

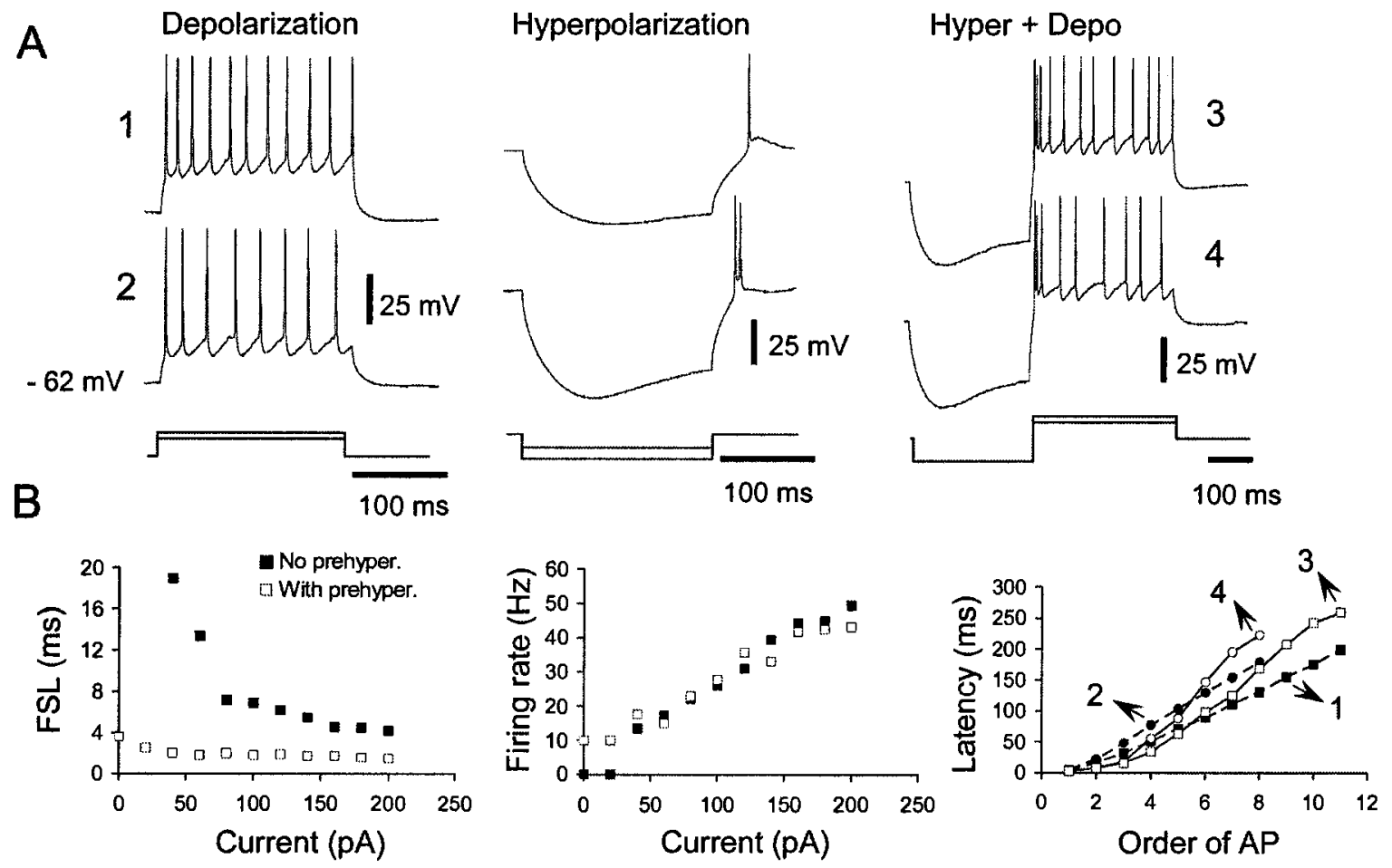

Figure 2.9. 
Figure 2.9. A firing pattern was changed from sustained to irregular by prehyperpolarization in a rebound neuron. A. Left panel: Responses to depolarizing current injection at two levels (top trace: $200 \mathrm{pA}$ and bottom trace: $150 \mathrm{pA}$ ), showing a regular firing pattern. Middle panel: Responses to two hyperpolarizing current pulses (top trace: $-50 \mathrm{pA}$ and bottom trace: $-150 \mathrm{pA}$ ). A rebound and spikes were generated after hyperpolarization. Right panel: Responses to the same levels of depolarizing current as shown in the left panel following a hyperpolarizing current of $-200 \mathrm{pA}$. Note irregular firing during the subsequent depolarization. B. Left and middle panels: The relationships of the first spike latency and firing rate to the current level with and without a prehyperpolarization. Right panel: The relationship between the latency of each spike in the train and the order of the spike without a pre-hyperpolarization (from traces 1 and 2 shown in $\mathbf{A}$ ) and with a pre-hyperpolarization (from traces 3 and 4 shown in $\mathbf{A}$ ). 


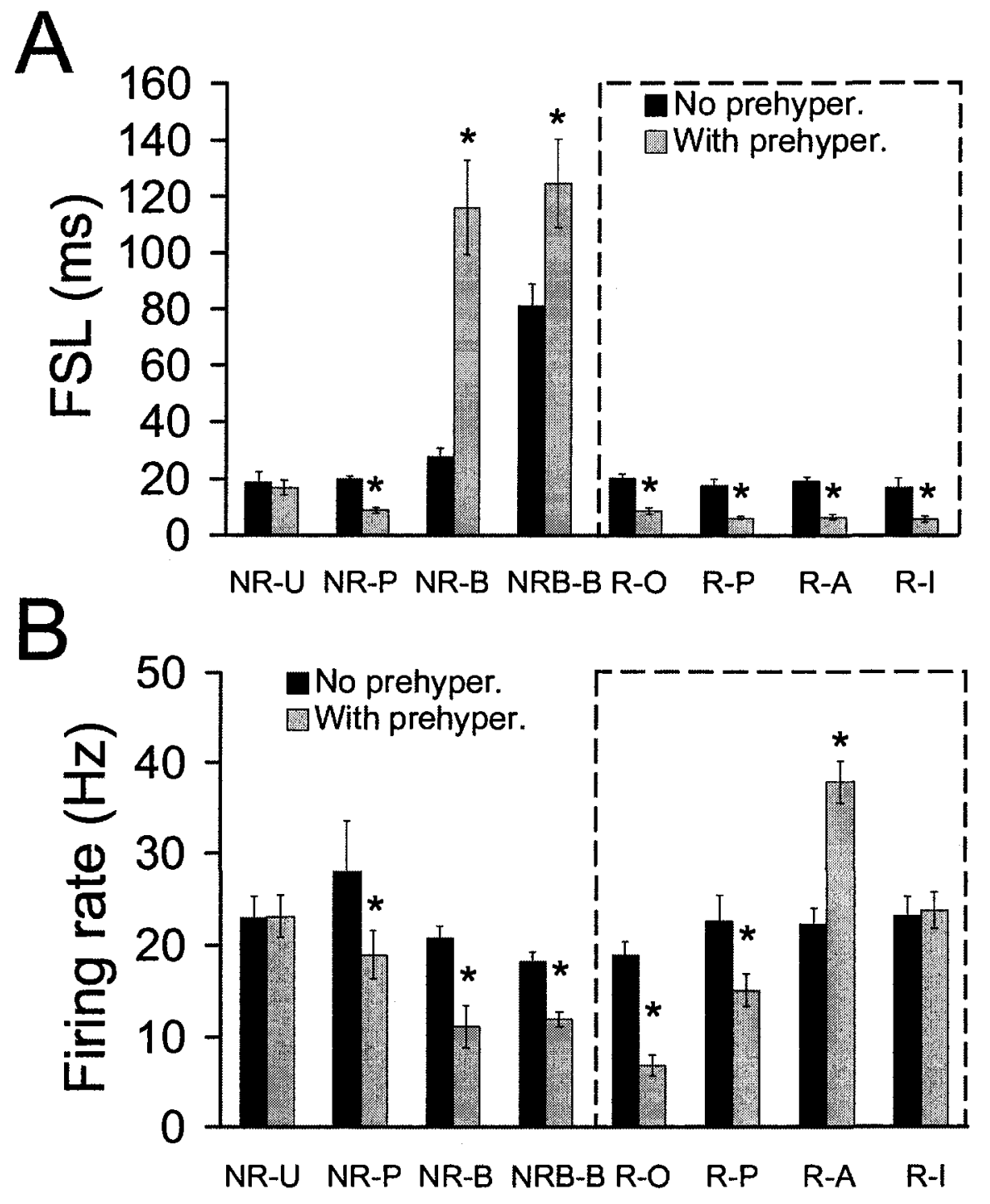

Figure 2.10. 


\section{Figure 2.10. Comparisons of the first spike latency and firing rate between} responses to depolarizing current injection with and without pre-hyperpolarization. A and B. The average first spike latencies and firing rates of 82 sustained and 6 buildup neurons. NR-: non-rebound cell; R-: rebound cell. The letter following "NR" or "R" represents the firing pattern during subsequent depolarization following prehyperpolarization. -U: the firing pattern was unchanged (sustained-regular or -adapting); -P: the firing pattern was changed to pauser; -B: the firing pattern was changed to buildup; $-\mathrm{O}$ : the firing pattern was changed to onset; -A: the firing pattern was changed from regular to adapting or the adapting firing became more evident; -I: the firing pattern was changed to irregular; B-B: the firing pattern remained buildup. ${ }^{*}$ indicates significant difference $(\mathrm{p}<0.05$, paired $t$-test $)$. 


\subsection{Discussion}

Findings from the present study indicate that there are three major intrinsic, physiological defined cell types in the ICD, classified as sustained-regular, sustainedadapting and buildup in response to positive current injection. Some of the regular and adapting neurons responded to negative current injection with a depolarizing rebound. But, different ICD neurons exhibited different changes in their firing pattern and membrane excitability in response to preceding hyperpolarization.

\subsubsection{Intrinsic membrane properties of ICD neurons around hearing onset}

Little change was found in intrinsic membrane properties of ICD neurons during postnatal days 10-19 except that the AP half-width of adapting neurons significantly decreased with age. In addition, all 3 firing patterns were observed in neurons of rats at these ages, although there was a tendency toward an increase in adapting firing and a decrease in regular firing with age. These results are consistent with previous studies of neurons in ICC and ICX (Peruzzi et al., 2000; Sivaramakrishnan and Oliver, 2001; Ahuja and $\mathrm{Wu}, 2007$ ), and auditory thalamus (Tennigkeit et al., 1998). The results from all of these studies indicate that for auditory midbrain and thalamus the distinct firing patterns have been established before hearing onset. The neuronal membrane properties around hearing onset (P10-12) to one week after hearing onset (P19) are relatively stable. On the other hand, principal neurons in the medial superior olive (MSO) undergo substantial changes in membrane properties during first week after the onset of hearing (Magnusson et al., 2005; Scott et al., 2005). The membrane input resistance, time constant and AP amplitude of MSO neurons decline considerably during this developmental period. These neurons possess some biophysical properties, i.e., low input resistance, onset-firing 
pattern, fast kinetics of synaptic responses, specialized for analyzing precise timing of auditory signals and comparing small binaural time differences (Magnusson et al., 2005; Scott et al., 2005). These properties are primarily mediated by low-threshold $\mathrm{K}^{+}$channels containing Kv1.1 subunits (Scott et al., 2005). The developmental changes in membrane and synaptic response properties in MSO principal cells have been attributed by Scott $e t$ al. (2005) to an increased expression of Kv1.1 containing channels. In ICD, however, there are very few cells labeled for Kv1.1 (Rosenberger et al., 2003). Results of this study indicates that modification of membrane excitability and firing pattern of ICD neurons in P10-19 by pre-hyperpolarization can not be attributed to developmental changes in membrane properties around hearing onset.

\subsubsection{Modification of membrane excitability by pre-hyperpolarization in non-rebound} neurons

In nearly half of the neurons sampled in this study, hyperpolarization did not result in a rebound depolarization. These non-rebound neurons showed either a sustained or a buildup firing pattern when they were injected by depolarizing current alone. Prehyperpolarization led to a modification of the membrane excitability in more than half of the non-rebound cells. With pre-hyperpolarization, these neurons had either pauser or buildup firing behavior in response to subsequent depolarization. For the neurons that showed a buildup pattern in response to depolarization alone (i.e., original buildup neurons), the pattern was not changed, but the first spike latencies became even longer than those in response to depolarization alone. The pauser or buildup neurons had some common characteristics. For example, there was a pause of firing after the 1st spike (for the pauser pattern) or a delay of firing (for the buildup pattern) following pre- 
hyperpolarization. The delay or pause was progressively decreased as the depolarizing current increased. A similar pauser/buildup pattern has been demonstrated for neurons in a number of auditory structures, e.g., the dorsal cochlear nucleus (DCN) (Manis, 1990), the ventral nucleus of the lateral lemniscus (Wu, 1999), ICC (Peruzzi et al., 2000; Sivaramakrishnan and Oliver, 2001), the external cortex of the IC (ICX) (Ahuja and Wu, 2007) and also neurons in other regions of the brain (Russier et al., 2003). Voltage-clamp studies have shown that the pause or long delay is due to removal of inactivation of a transient 4-aminopyridine (4-AP) sensitive A-type of $\mathrm{K}^{+}$current $\left(I_{\mathrm{A}}\right)$ by prehyperpolarization and then activation of $I_{\mathrm{A}}$ by a subsequent depolarization (Kanold and Manis, 1999, Sivaramakrishnan and Oliver, 2001). Direct evidence that $I_{\mathrm{A}}$ accounts for the pause or delay in firing has been obtained with current-clamp and voltage-clamp recordings from the same neuron by Sivaramakrishnan and Oliver (2001). They demonstrated that the amplitude of $I_{\mathrm{A}}$ and the pause or delay time increased in parallel as the pre-hyperpolarization was increased.

Examination of the kinetics of $I_{\mathrm{A}}$ in ICC neurons has shown that the current reached its peak amplitude in $5 \mathrm{~ms}$ and then decayed with a time course of $50-80 \mathrm{~ms}$ (Sivaramakrishnan and Oliver, 2001). Thus during the initial $\sim 50-100 \mathrm{~ms}$ of depolarization following pre-hyperpolarization, APs are not likely to be generated. APs can be produced only during the late period of depolarization with a larger depolarizing current (Fig. 2.1A, right panel). Neurons with a pauser or a buildup pattern showed different changes in their first spike latency in response to subsequent depolarization. The first spike latency in neurons displaying a pauser pattern became shorter, but those in neurons exhibiting a buildup pattern became longer. The length of the first spike latencies 
in neurons with a pauser/buildup pattern may depend on an interaction between the $I_{\mathrm{A}}$ and spike-generating mechanism of the cell (Kanold and Manis, 1999). If the mechanism for AP generation is similar for these neurons, the voltage dependence of activation and inactivation and/or kinetics of $I_{\mathrm{A}}$ in neurons having a pauser or a buildup pattern must be different, which may be the basis for the different first spike latency effects in these two groups of neurons. Various kinetics and voltage ranges for inactivation of $I_{\mathrm{A}}$ have been observed in different pyramidal cells in the DCN (Kanold and Manis, 1999). Kanold and Manis (2001) found a strong correlation between voltage levels for half-inactivation of $I_{\mathrm{A}}$ and voltage levels for the first spike latency shift in cochlear nucleus model cells. This suggests that the voltage range for half-inactivation of $I_{\mathrm{A}}$ might be also different in different ICD neurons. The ICD neurons that showed a buildup firing pattern may possess $I_{\mathrm{A}}$ whose half-inactivation is at a more positive voltage level than for ICD neurons that exhibited a pauser pattern. The pauser and buildup patterns can also be influenced by the kinetics of $I_{\mathrm{A}}$, i.e., the time constant of activation and inactivation of the $I_{\mathrm{A}}$ (Kanold and Manis, 2001). The neurons showing a buildup pattern may have $I_{\mathrm{A}}$ whose kinetics of activation and inactivation are faster than those of neurons displaying a pauser pattern.

Regardless of the mechanisms for generation of the pauser or buildup pattern, the membrane excitability for neurons showing either of the patterns was suppressed after pre-hyperpolarization because firing stopped after the first spike or was delayed during the early period of depolarization. If these neurons integrate a preceding inhibition with a subsequent excitation, the neuron might remain inhibited for the early period of the excitatory input. Therefore they may have output only during the late part of the 
stimulation. For neurons showing a pauser pattern after pre-hyperpolarization, the spike that appeared at the onset of the depolarization may code information about the onset of the subsequent excitatory stimulation.

\subsubsection{Modification of membrane excitability by pre-hyperpolarization in rebound} neurons

The rebound observed in this study was a broad depolarization immediately following hyperpolarization, which is very similar to the $\mathrm{Ca}^{2+}$-dependent rebound depolarization shown in neurons of the ICC and IC cortex (Smith, 1992; Sivaramakrishnan and Oliver, 2001; Ahuja and Wu, 2007). The generation of the rebound following hyperpolarization in ICD neurons is also consistent with the physiological characteristics of activation and inactivation of the low-threshold $\mathrm{T}$-type $\mathrm{Ca}^{2+}$ channel observed in other neurons, including ICC neurons (Rinzel et al., 1998; Fan et al., 2000; Sivaramakrishnan and Oliver, 2006). With membrane hyperpolarization, inactivation of low-threshold T-type $\mathrm{Ca}^{2+}$ channel is removed. Upon release of hyperpolarization the membrane potential returns to the resting level and the T-type $\mathrm{Ca}^{2+}$ channel is activated, resulting in a rebound depolarization.

In $\sim 29 \%$ of the rebound neurons tested the same level of depolarizing current produced more firing after pre-hyperpolarization. In other rebound neurons, subsequent depolarization either did not increase firing ( $\sim 29 \%$ neurons) or even decreased firing ( $42 \%$ neurons). Molineux et al. (2005) have reported that interaction between $I_{\mathrm{Ca}}$ and $I_{\mathrm{A}}$ in cerebellar stellate cells produces nonmonotonic spike latency-voltage relationship. I speculate that interaction between $I_{\mathrm{Ca}}$ and $I_{\mathrm{A}}$ with different strengths relative to each other 
in different ICD rebound cells might be one of the mechanisms that underlie different forms of modification of membrane excitability by pre-hyperpolarization.

As expected, some rebound neurons showed an increase in firing rate after prehyperpolarization, especially during the initial $200 \mathrm{~ms}$ of depolarization. The membrane became more excitable during the rebound; therefore more spikes were generated by subsequent depolarization. I speculate that a release of membrane hyperpolarization brings about activation of low-threshold T-type $I_{\mathrm{Ca}}$ which dominates over $\mathrm{I}_{\mathrm{A}}$, if there is any, and further depolarization leads to activation of voltage-dependent $\mathrm{Na}^{+}$channels and therefore more firing (Sekirmjak and du Lac, 2002). Interestingly, with this kind of modification, regular firing was changed to adapting, and original adapting firing became more evident. The adapting firing has been attributed to activation of $\mathrm{Ca}^{2+}$-activated $\mathrm{K}^{+}$ channels (Sivaramakrishnan and Oliver, 2001). During the rebound, voltage-gated $\mathrm{Ca}^{2+}$ channels are open, leading to more $\mathrm{Ca}^{2+}$ entering the neuron and an increase in intracellular $\mathrm{Ca}^{2+}$ concentration. This increase may further activate $\mathrm{Ca}^{2+}$-activated $\mathrm{K}^{+}$ channels, therefore resulting in less firing during the late period of the current pulse and producing adapting firing.

For some rebound neurons whose firing rate was decreased, i.e., those that produced only one spike (onset pattern) or one to two spikes followed by a long pause and then a spike train (pauser pattern) during subsequent depolarization, there may have been a considerable amount of $I_{\mathrm{A}}$ which dominated over T-type $I_{\mathrm{ca}}$ after the initial spikes. The long pause following pre-hyperpolarization is an indicator of activation of $I_{\mathrm{A}}$. For cells showing an onset pattern in this group, although no obvious pause appeared, the possibility of the presence of $I_{\mathrm{A}}$ can't be ruled out, because firing stopped after the $1^{\text {st }}$ 
spike. The membrane excitability of neurons in this group was greatly suppressed after pre-hyperpolarization.

For some other rebound neurons in which the firing rate was little changed over the initial $200 \mathrm{~ms}$ of depolarization, but the firing pattern became irregular, we speculate that interaction between $I_{\mathrm{A}}$ and low-threshold T-type $I_{\mathrm{Ca}}$ at relatively equal strengths leads to a cancellation of these two currents so that the firing rate remains unchanged. The irregular firing behavior over the subsequent current injection may be due to on-going variations in these two types of currents. Overall, for rebound neurons, prehyperpolarization modifies membrane excitability and alters firing pattern in different ways in different ICD neurons. Further voltage-clamp study is required to investigate whether the interplay of T-type $I_{\mathrm{Ca}}$ and $I_{\mathrm{A}}$ can produce different firing behavior in different ICD neurons.

For each of the rebound cells, the first spike latency in the firing after prehyperpolarization became much shorter but the latency was constant as the level of depolarization was increased. The short and constant first spike latency after prehyperpolarization may be attributable to faster kinetics for activation of T-type $I_{\mathrm{Ca}}$ than $I_{\mathrm{A}}$ (Molineux et al., 2005). The rate of the rising phase of the depolarization during subsequent positive current injection depends on the kinetics of activation of T-type $\mathrm{Ca}^{2+}$ and $\mathrm{Na}^{+}$currents. As both the T-type $\mathrm{Ca}^{2+}$ and $\mathrm{Na}^{+}$channels are voltage dependent, activation of these channels during the onset of subsequent depolarization would be facilitatory, leading to firing with a short and constant first spike latency.

In addition, generation of the rebound and associated spiking following hyperpolarization may also be attributable to activation of $I_{h}$, since blocking $I_{h}$ can reduce 
or completely eliminate the rebound and associated spiking in neurons of vestibular nucleus, ICC and hippocampus (Sekirnjak and du Lac, 2002; Koch and Grothe, 2003; Surges et al., 2006). However, in some other neurons, e.g., cerebellar deep nucleus neurons (Aizenmand and Linden, 1999) and unipolar brush neurons (Russo et al., 2007), postrema neurons (Funahashi et al., 2003) and ICD neurons (the present study), the rebound and associated spiking are still persistent after $I_{\mathrm{h}}$ is blocked, suggesting that activation of $I_{\mathrm{h}}$ is not necessary for initiation of the rebound in these neurons. The relation between activation of $I_{\mathrm{h}}$ and generation of the rebound may be different for neurons in different regions of the brain. Furthermore, whether generation of the rebound is dependent on activation of $I_{\mathrm{h}}$ may not be a simple "yes or no" answer. Activation of $I_{\mathrm{h}}$ can also shorten the latency of the rebound and spiking after pre-hyperpolarization (Funahashi et al., 2003; Fig. 2.3, the present study). Thus $I_{\mathrm{h}}$ may promote rather than determine generation of the rebound in some neurons. Detailed study is required to investigate how $I_{\mathrm{h}}$ affects generation of the rebound and associated spiking in ICD neurons.

\subsubsection{Functional implications of different modifications}

The ICD receives multiple inputs from various sources (Cant, 2005; Saldaňa and Merchán, 2005; Schofield, 2005). The main projection arises from neurons within the IC, including ICC, ICX and contralateral ICD, and neurons in the auditory cortex. Minor projections come from the cochlear nucleus, superior paraolivary nucleus, lateral nucleus of the trapezoid body, lateral superior olive, and nuclei of the lateral lemniscus. It has been suggested that some of these inputs are glutamatergic and others are GABAergic or glycinergic (Wu et al., 2004; Kelly and Caspary, 2005). Excitatory and inhibitory inputs 
may converge onto a single ICD neuron as shown in in vivo intracellular recordings in which complex interactions between excitatory and inhibitory sources in a single IC neuron in responses to acoustic stimulation emerge (Covey et al., 1996; Kuwada et al., 1997). If an ICD neuron receives an excitatory input preceded by an inhibitory input, which may be indeed the case (Covey et al., 1996; Kuwada et al., 1997), the neuron would be hyperpolarized before it is depolarized. The membrane excitability and firing behavior of ICD neurons during the response to the late excitatory input can be different from those without any prior inhibitory input. In fact, the data from this study show that the firing pattern, first spike latency, and firing rate are changed after prehyperpolarization in about $80 \%$ of the ICD neurons, indicating a high probability of such modifications.

I expect that the excitatory rebound would allow summation with another excitatory input that arrives later than the inhibitory input, but simultaneously with the rebound. Thus the neuron would be more likely to fire. Some of our rebound neurons showed such an enhancement in response to a subsequent depolarization after prehyperpolarization. This pattern of excitatory-inhibitory interaction has been proposed as a possible mechanism for selective responses to sound duration of a certain range (Covey and Casseday, 1999). Indeed, whole-cell patch clamp recordings revealed early inhibition followed by an excitatory rebound in IC neurons that were selective to sound duration (Covey et al., 1996). Thus, the rebound is an important feature for enhancing membrane excitability and firing probability in duration tuned neurons (Casseday and Covey, 1999).

The distinct changes encountered in different neurons may be related to specific modes of processing different aspects of auditory information. Many ICD neurons in the 
rat have been shown to respond selectively to long duration sounds, so called long-pass neurons (Pérez-González et al., 2006). The results of the study by Pérez-González et al (2006) further demonstrated that when the sound level is increased, the shortest duration a neuron can respond to, i.e., the cut-off duration, is unchanged or becomes longer or shorter in different neurons. The authors hypothesize that different degrees of growth of excitation and preceding synaptic inhibition may differentially affect the cut-off duration in different neurons when the sound level is increased. The data from the present study provide additional support for this model. The three types of modification of firing after pre-hyperpolarization in the rebound neurons can explain why cut-off duration and response latency changed differentially in different neurons. The neurons whose cut-off duration remained unchanged when sound level was increased may correspond to the neurons in which the firing rate did not change substantially after pre-hyperpolarization (Fig. 2.9). The neurons whose cut-off duration was prolonged as sound level was increased may correspond to the neurons in which the firing rate decreased after prehyperpolarization (Fig. 2.8). The neurons whose cut-off duration was shortened as sound level was increased may correspond to the neurons whose firing rate increased after prehyperpolarization (Fig. 2.7).

In addition, for all the rebound neurons and neurons whose firing was changed to a pauser pattern, the first spike latency became shorter after pre-hyperpolarization, but was constant as the level of depolarization was increased. These neurons may be involved in coding information about the onset of subsequent stimulation. On the other hand, for neurons whose firing remained unchanged (i.e., remained sustained or buildup) or changed from sustained to buildup, the first spike latency was decreased as the current 
level was increased. These neurons may code no information about the onset of subsequent stimulation, but still provide information about the magnitude of stimulation through an increase in firing rate. In summary, the distinct modifications of membrane excitability and firing by a preceding hyperpolarization in ICD neurons provide various mechanisms for processing specific aspect of auditory information. 


\section{Chapter 3}

\section{Contribution of calcium to membrane excitability of neurons in the rat's dorsal cortex of the inferior colliculus}

\subsection{Introduction}

Anatomical connections of ICD with higher and lower parts of the central auditory system, and with other parts of the IC suggest that the ICD may play a special role in processing and gating auditory information. In vivo electrophysiological recordings have shown that responses from ICD neurons are sluggish, have long latencies and show broad tuning to auditory stimuli (Aitkin et al., 1994; Syka et al., 2000; Zhang and Kelly, 2006). ICD neurons also possess response characteristics that are likely related to the selective processing of auditory information, e.g., detecting complex and novel auditory stimuli (Pérez-González et al., 2005), or a certain duration of sound (PérezGonzález et al., 2006). The study of Chpter 2 has shown that most ICD neurons responded to depolarizing current injection with sustained firing (Sun and $\mathrm{Wu}, 2007$ ). Half of these neurons had a rebound depolarization upon release of membrane hyperpolarization. The membrane excitability and firing behavior of the rebound neurons in ICD were altered in response to subsequent depolarization following a preceding hyperpolarization. The rebound depolarization has been shown to be a result of activation of $\mathrm{Ca}^{2+}$ channels in IC neurons (Smith, 1992; Sivaramakrishnan and Oliver, 2001; Ahuja and $\mathrm{Wu}, 2007)$. In addition to $\mathrm{Ca}^{2+}$ mediated rebound, $\mathrm{Ca}^{2+}$ spikes and $\mathrm{Ca}^{2+}$-activated $\mathrm{K}^{+}$ current mediated afterhyperpolarization (AHP) in IC neurons have also been implicated (Smith, 1992; Li et al., 1998; Sivaramakrishnan and Oliver, 2001; Ahuja and Wu, 2007). 
All these results suggest that $\mathrm{Ca}^{2+}$ plays a significant role in regulating membrane excitability and governing firing behavior of IC neurons.

The purpose of this study was to investigate further how membrane excitability of ICD neurons was regulated by $\mathrm{Ca}^{2+}$ mediated AHP, spikes and rebound. I also investigated what level and duration of hyperpolarization were required for generation of the rebound and associated spikes. The results suggest that the $\mathrm{Ca}^{2+}$ mediated events play important roles in regulating membrane excitability not only when the neuron receives excitatory inputs, but also when it returns to the resting state following inhibitory inputs.

\subsection{Materials and Methods}

\subsubsection{Preparation of brain slices}

Same as that described in Chapter 2.

\subsubsection{Solutions and drugs}

Unless indicated otherwise, a standard ACSF was used. It contained (in mM): 129 $\mathrm{NaCl}, 3 \mathrm{KCl}, 1.2 \mathrm{KH}_{2} \mathrm{PO}_{4}, 2.4 \mathrm{CaCl}_{2}, 1.3 \mathrm{MgSO}_{4}, 20 \mathrm{NaHCO}_{3}, 3$ HEPES, and 10 glucose at a pH of 7.4 and had an osmolarity of 290-310 mOsm $/ \mathrm{kg}$. In $\mathrm{Ca}^{2+}$-free ACSF, $\mathrm{Ca}^{2+}$ was omitted and replaced by $\mathrm{Mg}^{2+}$. When $\mathrm{NiCl}_{2}(100 \mu \mathrm{M})$ was added to the ACSF, $\mathrm{KH}_{2} \mathrm{PO}_{4}$ was removed to prevent precipitation. In addition, in some experiments, ion channel blockers were used. These included $1 \mu \mathrm{M}$ tetrodotoxin (TTX), $10 \mathrm{mM}$ tetraethylammonium chloride (TEA-Cl), $2 \mathrm{mM}$ 4-aminopyridine (4-AP), $100 \mathrm{nM}$ apamin and $200 \mu \mathrm{M} \mathrm{CdCl}_{2}$. All drugs were obtained from Sigma Aldrich Company.

\subsubsection{Whole-cell patch clamp recording}


Electrodes for whole-cell patch clamp recordings were prepared from thin-walled glass pipettes (Kimax-51; Kimble, Vineland, NJ) with a two-stage vertical puller (PP830, Narishige, Tokyo, Japan). The electrode was filled with an internal solution containing (in mM): $130 \mathrm{~K}$-gluconate, 0.6 EGTA, 10 HEPES, $2 \mathrm{MgCl}_{2}, 5 \mathrm{KCl}, 2 \mathrm{ATP}, 0.3 \mathrm{GTP}$. The $\mathrm{pH}$ of the solution was adjusted to 7.25 with $\mathrm{KOH}$ and the osmolarity was $280-290$ $\mathrm{mOsm} / \mathrm{kg}$. The resistance of the patch electrode was 4-7 M 2 . Whole-cell patch clamp recordings were made by an EPC-8 patch-clamp amplifier (HEKA, Darmstadt, Germany). ICD neurons were visualized under a Zeiss Axioskop microscope by a 40X water immersion objective with Hoffman modification contrast. The membrane potentials reported in this study were corrected with the junction potential. Series resistances were in the range of $15-25 \mathrm{M} \Omega$ and were compensated by $50-70 \%$. If the series resistance changed by more than $15 \%$ of the initial value during the recording, the data were discarded. Signals were filtered at $5 \mathrm{kHz}$, digitized at $10 \mathrm{kHz}$ by Digidata 1200 interface, acquired by pClamp 6 , and analyzed off-line by pClamp 8 (Axon Instruments, CA).

\subsubsection{Data analysis}

All measurements were made from the recordings at least 6-8 min after establishing a whole-cell configuration and showing a stable resting potential. Neurons with resting potentials more negative than $-55 \mathrm{mV}$ were accepted and used for further analysis. The spike half-width was measured at $1 / 2$ amplitude of an action potential (AP). The initial duration of the AHP was determined by the time between the peak of the AP and the lowest level of the AHP. The amplitude of AHP was measured from the beginning of the spike to the lowest level of the AHP. The AHP half-width was measured at $1 / 2$ amplitude of the AHP. The changes in voltage elicited via hyperpolarizing current 
injection were measured between the resting membrane potential and the peak membrane potential. These values were then used to plot the current-voltage (IV) curve. The slope of the IV curve derived from voltage changes elicited by $200 \mathrm{~ms}$ current injections from 0 to $-80 \mathrm{pA}$ was used to determine the input resistance $(\mathrm{M} \Omega)$ of a cell membrane.

The first spike latency (FSL) of the anode break action potentials was measured from the release of hyperpolarizing current to the peak of the first spike. The slope of the rebound was measured from the baseline to the beginning of the spike (dashed lines in Fig. 3.5A, insert). Normalized FSL, expressed as a percent, was calculated as a ratio of the FSL at each hyperpolarizing current level to that of the 1st spike generated by the smallest hyperpolarizing current that just elicited one anode break spike. With the same principle, I normalized the slope of rebound depolarization and the threshold of anode break action potentials.

The amplitude of the rebound was measured as the difference between the resting membrane potential and the peak depolarization. The area of the rebound depolarization was measured using the statistics function of Clampfit 8 (Axon Instruments). The maximum rebound was defined as the rebound that reached $80 \%$ of the peak amplitude. The rise time of the rebound was measured as the time from $10 \%$ to $90 \%$ of the peak value. The decay time of the rebound was measured as the time from $90 \%$ to $10 \%$ of the peak value. Half-width of the rebound was measured at $1 / 2$ amplitude of the rebound. The amplitude, area, rising time, decay time and half-width of the rebound were normalized to those of the maximum rebound evoked by a current pulse of $-300 \mathrm{pA}$ for $585 \mathrm{~ms}$.

Numerical averages are presented as means \pm SEMs. Statistical significance was evaluated by either Student's paired or unpaired $t$ tests, or a one-way ANOVA with a post 
hoc test, and the minimum criterion for statistical significance was set at $p<0.05$.

\subsection{Results}

Whole-cell patch clamp recordings were obtained from 82 ICD neurons for the present study. All the neurons displayed sustained firing in response to depolarizing current injections and had a stable resting membrane potential more negative than -55 $\mathrm{mV}$ (range: -56 to $-64 \mathrm{mV}$; mean: $-60.3 \pm 0.3 \mathrm{mV}$ ) throughout the recording.

\subsubsection{Afterhyperpolarization (AHP) mediated by calcium conductance}

Most sampled ICD neurons (91.5\%, 75 of 82 cells) had an AHP following falling phase of an AP. Three types of AHP were observed in these neurons (Fig. 3.1A). The first type was characterized by a slow and long hyperpolarization (Fig. 3.1 $\mathrm{A}_{1}$, solid triangle) and classified as slow AHP (sAHP). The average half-width and initial duration of the AHP were $50.3 \pm 3.5 \mathrm{~ms}$ and $23.3 \pm 1.1 \mathrm{~ms}$ respectively $(\mathrm{n}=59)$. The sAHP was the most common type and found in $78.7 \%$ of the ICD neurons that showed an AHP. The second type was characterized by a fast and short hyperpolarization (Fig. 3.1 $\mathrm{A}_{2}$, open triangle), and was classified as fast AHP (fAHP). The half-width and initial duration of the fAHP were $30.1 \pm 4.8 \mathrm{~ms}$ and $4.8 \pm 0.8 \mathrm{~ms}$ respectively $(\mathrm{n}=11)$, values that were significantly shorter than those for the SAHP $(\mathrm{p}<0.01)$ (Fig. 3.1B and C). The fAHP was found in $14.7 \%$ of the ICD neurons showing an AHP. The $3^{\text {rd }}$ type of AHP was characterized by a complex waveform with both fast and slow components, and classified as combined AHP (cAHP) (Fig. 3.1 $A_{3}$ ). For these cells, an early, fast and short component (open triangle) was typically followed by a late, slow and long one (solid 
triangle). The half-width and initial duration of the cAHP were $69.2 \pm 9.4 \mathrm{~ms}$ and $31.0 \pm 3.9$ ms respectively $(n=5)$, values that were significantly longer than those for the fAHP $(p<0.01)$, but not different from the sAHP $(p>0.05)$ (Fig. 3.1B and C). The cAHP was found in only $6.7 \%$ of the neurons that had an AHP. In regarding to the amplitude of the AHP, however, no significant difference was observed among the neurons with all three types of AHP ( $p>0.05)$ (Fig. 3.1D).

\subsection{2 $\mathrm{Ca}^{2+}$-activated $\mathrm{K}^{+}$current mediated $\mathrm{sAHP}$}

As the SAHP was observed in the majority of ICD neurons, its ionic basis and pharmacology were further investigated. When the slice was bathed in calcium-free $\mathrm{ACSF}$, the sAHP was almost abolished $(\mathrm{n}=6)$ (Fig. $3.2 \mathrm{~A}_{1}$, arrow). At the same time, the firing of the cell increased compared to firing of the same cell in response to the same level of depolarizing current pulse with the slice in normal ACSF (Fig. 3.2A $\mathrm{A}_{2}$ ). I further investigated the role of small conductance $\mathrm{Ca}^{2+}$-mediated $\mathrm{K}^{+}\left(\mathrm{SK}_{\mathrm{Ca}}\right)$ channels in generation of the sAHP $(n=4)$. Bath application of apamin $(100 \mathrm{nM})$, a selective $\mathrm{SK}_{\mathrm{Ca}}$ channel blocker, decreased the amplitude (from $13.5 \mathrm{mV}$ to $10.5 \mathrm{mV}$ ) and half-width (from $79.6 \mathrm{~ms}$ to $67.9 \mathrm{~ms}$ ) of the sAHP (Fig. $3.2 \mathrm{~B}_{1}$, arrow). The firing of the neuron was also slightly increased (Fig. $3.2 \mathrm{~B}_{2}$ ) by apamin. These results suggest that the generation of the sAHP partially depends on a $\mathrm{Ca}^{2+}$-activated $\mathrm{K}^{+}$current which flows through $\mathrm{SK}_{\mathrm{Ca}}$ channels.

\subsubsection{Calcium-mediated depolarization and spikes}


When subthreshold depolarizing currents were injected, a slow depolarizing hump was found in $37.8 \%$ ( 31 of 82 cells) ICD neurons. A representative example is illustrated in Figure $3.3 \mathrm{~A}_{1}$. When a $90 \mathrm{pA}$ current pulse was injected, a hump appeared at the beginning of the depolarization (Fig. $3.3 \mathrm{~A}_{1}$, arrow). I then plotted the voltage changes at the peak of the hump (solid circles) and the steady level of the depolarization (open circles) against the current level (Fig. 3.3 $\mathrm{A}_{2}$ ). The difference between the two curves represented the hump amplitude. The hump was elicited at current levels as low as $50 \mathrm{pA}$ and became larger as the current level increased. When a current pulse was larger than 90 pA, multiple spikes were generated, but the hump could no longer be observed (Fig. 3.3 $\mathrm{A}_{1}$, upper trace). In order to examine the possibility that $\mathrm{Ca}^{2+}$ was mediating this slow depolarizing hump, the slice was bathed in $\mathrm{Ca}^{2+}$-free $\operatorname{ACSF}(\mathrm{n}=3)$. The hump was almost eliminated after removal of $\mathrm{Ca}^{2+}$ (Fig. 3.3 $\mathrm{A}_{1}$, arrowhead), indicating that the hump is mediated by $\mathrm{Ca}^{2+}$ conductance.

To test whether $\mathrm{Ca}^{2+}$ also contributes to generation of spikes in ICD neurons, including those showing no obvious depolarizing hump, the blockers for $\mathrm{Na}^{+}$(TTX), $\mathrm{K}^{+}$ (TEA and 4-AP) and $\mathrm{Ca}^{2+}\left(\mathrm{CdCl}_{2}\right)$ conductance were applied $(\mathrm{n}=3)$. An example is shown in Figure 3.3B. In ACSF, a depolarizing current injection (70 pA) induced a train of spikes. After application of TTX, TEA and 4-AP, typical $\mathrm{Na}^{+}$spikes were abolished, but regenerative smaller and broader spikes were revealed with a higher level of current injection (150 pA). After addition of $\mathrm{CdCl}_{2}$ to the above drug solution, these spikes were eliminated. The train of $\mathrm{Na}^{+}$spikes returned after washing the slice with ACSF. The results indicate that $\mathrm{Ca}^{2+}$ conductance is involved in generation of APs in ICD neurons. 


\subsubsection{Calcium mediated rebound depolarization following hyperpolarization}

In more than half of the ICD neurons $(54.9 \%, 45$ of 82 cells) a broad rebound depolarization was elicited following hyperpolarization (Fig. 3.4A, upper panel, arrow). If the rebound was large enough, an anode break AP (sometimes 2 or more APs) was generated from the rebound (Fig. 3.4A, upper panel). Anode break APs were also generated from some neurons that did not show rebound (Fig. 3.4A, lower panel). However, the anode break APs in neurons having the rebound had some characteristics quite different from those in neurons without rebound depolarization (Fig. 3.4B). For example, the latency of the anode break AP in rebound-neurons $(60.6 \pm 6.1 \mathrm{~ms}, \mathrm{n}=20)$ was much shorter than that in non-rebound neurons $(114.6 \pm 11.8 \mathrm{~ms}, \mathrm{n}=10, \mathrm{p}<0.01)$. A hyperpolarization required to elicit anode break APs in rebound neurons $(-18.1 \pm 2.1 \mathrm{mV}$, $\mathrm{n}=20)$ was less negative than that in non-rebound cells $(-30.2 \pm 3.8 \mathrm{mV}, \mathrm{n}=10, \mathrm{p}<0.05)$. In addition, in rebound neurons the half-width of the APs $(1.17 \pm 0.07 \mathrm{~ms}, \mathrm{n}=45)$ was smaller than that in non-rebound cells $(1.53 \pm 0.08 \mathrm{~ms}, \mathrm{n}=16)(\mathrm{p}<0.05)$. The rebound neurons had lower membrane input resistance $(253.7 \pm 16.4 \mathrm{M} \Omega, \mathrm{n}=45)$ than non-rebound ICD neurons $(335.5 \pm 14.9 \mathrm{M} \Omega, \mathrm{n}=16, \mathrm{p}<0.01)$. The resting membrane potential, membrane time constant and AP amplitude were similar for rebound and non-rebound cells $(p>0.05)$.

To investigate further the ionic basis and pharmacology of the rebound, I tested the effects of removal of $\mathrm{Ca}^{2+}$ on generation of the rebound $(\mathrm{n}=6)$. Figure $3.4 \mathrm{C}$ shows an example. Following a hyperpolarization induced by a current injection $(-160 \mathrm{pA})$, a rebound depolarization (arrow) and an anode break spike (arrowhead) were elicited. Removal of $\mathrm{Ca}^{2+}$ from the ACSF totally eliminated the rebound and the associated AP. 
The results indicate that the rebound is mediated by influx of $\mathrm{Ca}^{2+}$. Next, to assess which type of calcium channels contribute to the rebound, I examined the effect of $\mathrm{Ni}^{2+}$ which has been shown to block T-type calcium channel selectively ( $\mathrm{n}=4)$. Perfusion with $\mathrm{NiCl}_{2}$ $(100 \mu \mathrm{M})$ reduced the rebound by about $80 \%$ and this effect was reversible after washing the slice with ACSF (Fig. 3.4D). The results suggest that T-type calcium current primarily mediates the rebound depolarization. In addition to T-type calcium current, the rebound depolarization in some other neurons have been demonstrated to be attributable to hyperpolarization-activated current, $I_{\mathrm{h}}$ (Aizenman and Linden, 1999; Koch and Grothe, 2003; Surges et al., 2006). In the study of last chapter, I applied ZD7288, the $I_{\mathrm{h}}$ blocker, and demonstrated that activation of $I_{\mathrm{h}}$ is not necessary for generation of the rebound in ICD neurons (Sun and Wu, 2007). In this study, I also noticed that when a rebound was generated after injection of a hyperpolarizing current of -40 to $-60 \mathrm{pA}$, there was no obvious voltage sag during the hyperpolarization. As usually the voltage sag is a reflection of activation of $I_{\mathrm{h}}$ (Bal and Ortel, 2000; Koch and Grothe, 2003), the results further support the notion that generation of the rebound in ICD neurons does not rely on activation of $I_{\mathrm{h}}$.

In this study the recordings were made from the rat's brain slices of P10-P19. In order to rule out the possibility that animal age might influence the physiological properties of the rebound-neurons, therefore the characteristics of the $\mathrm{RD}$ itself, I compared membrane properties of the neurons in three age groups, i.e., P10-12, just before hearing onset; P13-15 and P16-19, several days after hearing onset. The results show that the resting potential, input resistance, time constant, and amplitude, threshold and half-width of APs of the rebound- neurons among the three groups were very similar 
(Table 3.1) $(\mathrm{P}>0.05)$. In addition, the average $\mathrm{RD}$ amplitude of the neurons among the three groups was also similar $(\mathrm{P}>0.05$, Table 3.1$)$.

\subsubsection{Generation of the rebound and anode break APs depended on magnitude of preceding hyperpolarization}

To examine how generation of the anode break AP that arose on the rebound was influenced by a preceding hyperpolarization, I injected a series of hyperpolarizing current pulses at different levels $(n=20)$. I investigated how FSL and threshold, and the slope of the rebound rising phase were affected by the level of hyperpolarization. Figure $3.5 \mathrm{~A}$ shows responses recorded from a neuron to 4 levels of hyperpolarizing current. Lower levels of hyperpolarizing current injection ( -60 and $-80 \mathrm{pA}$ ) induced only small and broad rebound depolarizations (Fig. 3.5A, traces 1 and 2). As the level of hyperpolarizing current increased to $-100 \mathrm{pA}$ or higher, the rebound became larger and passed the threshold for an AP. As a result, anode break action potentials were generated at the early phase of the rebound. I noticed that as the hyperpolarizing current increased, the slope of the rebound became larger and larger, the anode APs were generated earlier and earlier (Fig. 3.5A, traces 3 and 4), and the threshold of the AP was lower and lower (Fig. 3.5A, arrowheads in the insert). I plotted the FSL and threshold of the anode break AP, and the slope of the rebound rising phase against the level of hyperpolarizing current respectively (Fig. 3.5B, C and D). The three parameters were closely related to the current level. As the hyperpolarizing current level was increased from $-80 \mathrm{pA}$ to $-300 \mathrm{pA}$, the latency (from $57.6 \pm 6.1 \mathrm{~ms}$ to $32.2 \pm 3.4 \mathrm{~ms}$ ) and threshold (from $-46.5 \pm 2.6 \mathrm{mV}$ to $-50.0 \pm 1.8 \mathrm{mV}$ ) of the anode AP were progressively decreased, and the slope of the rebound gradually 
increased (from $0.97 \pm 0.27 \mathrm{mV} / \mathrm{ms}$ to $2.43 \pm 0.19 \mathrm{mV} / \mathrm{ms}$ ). It is worthwhile mentioning that the threshold of the AP that arose on the rebound was lower than that of the typical APs that were generated by depolarizing current injection $(-43.2 \pm 1.1 \mathrm{mV}$, paired $t$-test, $\mathrm{p}<0.05)$.

Next, I examined whether the changes in latency and threshold of the anode break AP were significantly correlated with the rebound slope. I plotted normalized FSL and threshold of the $1^{\text {st }}$ anode break AP against the normalized slope of the rebound for 9 neurons (Fig. 3.5E and F). The results demonstrate a strong correlation $(\mathrm{p}<0.001)$ between the FSL and the rebound slope, and also the threshold of the $1^{\text {st }}$ anode break AP and the rebound slope. Taken together, these results suggest that generation of the anode break APs depended not only on the amplitude of the rebound depolarization, but also on the rate of the cell membrane depolarization during the rebound.

To determine how generation of the rebound depolarization is related to the level of hyperpolarization, I applied TTX $(1 \mu \mathrm{M})$ to eliminate anode break spikes, a procedure that allowed us measure the amplitude and area of the rebound. Figure $3.6 \mathrm{~A}$ shows responses of a neuron to a series of hyperpolarizing current pulses of $200 \mathrm{~ms}$ from 0 to $400 \mathrm{pA}$ with increments of $-20 \mathrm{pA} / \mathrm{step}$ after application of TTX. When the hyperpolarizing current was $-50 \mathrm{pA}$, a noticeable rebound $(4.5 \mathrm{mV})$ was induced. As the current was further increased up to $-100 \mathrm{pA}$, the amplitude of the rebound increased greatly (up to $20.8 \mathrm{mV}$ ). However, further increasing in hyperpolarization from $-100 \mathrm{pA}$ to $-400 \mathrm{pA}$ did not result in any larger rebound (Fig. 3.6B, left panel). The mean maximum amplitude of the rebound was $18.6 \pm 0.9 \mathrm{mV}(\mathrm{n}=8)$. In addition, I measured the total area of the rebound in order to quantify the rebound further. The rebound area 
increased substantially with the increment of hyperpolarizing current pulse at lower levels (from $-50 \mathrm{pA}$ to $-70 \mathrm{pA}$ ) (Fig. 3.6B, middle panel). Further increasing current level up to $-400 \mathrm{pA}$ led to gradual, but slight increase in the area of the rebound. The mean maximum area of the rebound was $1021.9 \pm 72.2 \mathrm{mV}^{*} \mathrm{~ms}(\mathrm{n}=8)$. The mean normalized amplitude and area of the 8 rebound cells were plotted as a function of current levels (Fig. 3.6C, left and middle panels), displaying similar relationships as those for the neuron shown in Figure 3.6B. These results suggest that there is a "threshold" (i.e., hyperpolarization level) for generation of a rebound. Once the hyperpolarization is larger enough, the rebound depolarization will not further augment with increasing in negative current level.

To investigate how kinetics and time course of the rebound changed as the hyperpolarizing current level increased, I measured rise and decay time, and half width of the rebound. I then plotted them against the current level respectively (Fig. 3.6B, right panel). The rise and decay time, and the half width decreased substantially with the increasing current at lower levels $(<-100 \mathrm{pA})$, but had little change as the current level was larger than $-100 \mathrm{pA}$. The normalized data of rebound kinetics for the 8 neurons displayed similar relationships (Fig. 3.6C, right panel), an outcome consistent with the relationship of the rebound magnitude to the level of hyperpolarization.

\subsubsection{Generation of the rebound and anode break APs depended on duration of preceding hyperpolarization}

To investigate how generation of the rebound and anode break APs depended on duration of hyperpolarization, I applied a fixed level of negative current with increasing 
duration of the current pulse from $25 \mathrm{~ms}$ up to $1005 \mathrm{~ms}$ in $20 \mathrm{~ms} / \mathrm{step}(\mathrm{n}=7)$. Figure 3.7A shows an example illustrating the relationship of the rebound and anode break APs to the duration of hyperpolarization at a level of $-80 \mathrm{pA}$. A noticeable rebound $(\sim 7.5 \mathrm{mV})$ was evoked by a hyperpolarization of $85 \mathrm{~ms}$ (arrows). As the duration of the current pulse was increased to $165 \mathrm{~ms}$ or longer, anode break APs were elicited on the rebound. I measured the latencies of the APs (Fig. 3.7B, insert), and plotted them against the current duration. As the hyperpolarization was gradually lengthened from $165 \mathrm{~ms}$ to $400 \mathrm{~ms}$, the latencies of the anode break APs progressively decreased. However, when the hyperpolarization was longer than $400 \mathrm{~ms}$, the AP latencies did not further decrease (Fig. 3.7B, left panel). The average latencies from 7 cells also had a similar relationship (Fig. 3.7B, right panel). These results suggest that a longer hyperpolarization leads to an earlier anode break AP, and once the hyperpolarization is long enough, the anode break AP occurs at a relatively constant time on the rebound.

To demonstrate further how generation of the rebound, its kinetics and time course were affected by duration of the hyperpolarization, I measured amplitude, area, rise and decay time, and half-width of the rebound after blocking the anode break APS with TTX. Figure $3.8 \mathrm{~A}$ shows responses of a representative neuron to injection of hyperpolarizing current of $-80 \mathrm{pA}$ for $25 \mathrm{~ms}$ up to $585 \mathrm{~ms}$ (in $20 \mathrm{~ms} / \mathrm{step}$ ). A noticeable rebound was evoked by a hyperpolarizing current pulse as short as $65 \mathrm{~ms}$ (Fig. 3.8A, arrows). As the duration of the current pulse was further increased up to $205 \mathrm{~ms}$, the amplitude of the rebound was greatly augmented. However, a further increase in duration of the current pulse from 205 to $585 \mathrm{~ms}$ did not lead to a larger rebound (Fig. 3.8B, left panel). In other words, once the hyperpolarization was sufficiently long, the rebound 
reached its maximum level. Very similar relations of the changes in the rebound area to current duration were also obtained (Fig. 3.8B, middle panel). The rise and decay time of the rebound, and its half-width for this neuron gradually decreased as the duration of the current pulse was increased from $65 \mathrm{~ms}$ to $\sim 200 \mathrm{~ms}$, but remained at almost the same level for current pulse longer than $200 \mathrm{~ms}$ (Fig. 3.8B, right panel). The group data from 6 neurons were normalized and are presented in Figure $3.8 \mathrm{C}$. The general relations of the amplitude, area and kinetics of the rebound to the duration of hyperpolarizing current were similar to those of the example shown in Figure 3.8B.

\subsubsection{Level and duration of hyperpolarization required for generation of minimum and maximum rebound}

To estimate the level and duration of hyperpolarization required to elicit minimum or maximum rebound, hyperpolarizing current was injected in a fixed step from -40 to $-300 \mathrm{pA}(-20 \mathrm{pA} / \mathrm{step})$ for varying periods of time from $25 \mathrm{~ms}$ up to $1005 \mathrm{~ms}$ in $20 \mathrm{~ms} / \mathrm{step}$ in 6 neurons. Figure $3.9 \mathrm{~A}$ shows responses of a neuron to injections of current pulses of $-40 \mathrm{pA}$ (upper traces) and $-120 \mathrm{pA}$ (lower traces) for varying durations. When a current pulse was injected at lower levels, a longer time was required to elicit a discernible (i.e., minimum) rebound than when higher levels of current were injected. For example, with a current pulse of $-40 \mathrm{pA}$, the current injection time had to be at least 165 $\mathrm{ms}$ to evoke a rebound of $2 \mathrm{mV}$ (Fig. 3.9A , arrowhead), whereas with a current pulse of $-120 \mathrm{pA}$, the current injection for only $45 \mathrm{~ms}$ could elicit a rebound of $4.5 \mathrm{mV}$ (Fig. $3.9 \mathrm{~A}_{2}$, arrowhead). Similar relations of the current duration and level to maximum rebound were also observed. For example, at least $525 \mathrm{~ms}$ of the low level of current 
pulse $(-40 \mathrm{pA})$ was required to evoke a maximum rebound (Fig. 3.9 $\mathrm{A}_{1}$, arrows), whereas only $145 \mathrm{~ms}$ of the current pulse of $-120 \mathrm{pA}$ was required to generate a maximum rebound (Fig. 3.9 $\mathrm{A}_{2}$, arrows). A comparison of the relationships between the amplitude of the rebound and current pulse duration at two levels of current injection for this neuron is shown in Figure 3.9B. For the current injection of $-40 \mathrm{pA}$, the amplitude of the rebound gradually increased to a maximum level as the current duration was lengthened from 165 ms to $665 \mathrm{~ms}$. Whereas, the amplitude of the rebound rapidly increased to a maximum level when the current duration was increased from $45 \mathrm{~ms}$ to $125 \mathrm{~ms}$. It is worthwhile mentioning that although a longer period of time for lower level current was needed to reach the maximum rebound, the amplitude of the maximum rebound was similar (e.g., $18.5 \mathrm{mV}$ for $-40 \mathrm{pA}$ and $21.5 \mathrm{mV}$ for $-120 \mathrm{pA}$ ) at all levels of current injection.

Summary data about the relationships between the minimum or maximum rebound to the duration and level of hyperpolarization for six cells are illustrated in Figure 3.9C and D. For eliciting a minimum rebound $(\sim 1.5-2 \mathrm{mV})$, with the current levels between -40 to $-200 \mathrm{pA}$, longer hyperpolarizing current was required for smaller current, and vise versa (e.g., $175.0 \pm 11.3 \mathrm{~ms}$ was required for $-40 \mathrm{pA}$ and $38.3 \pm 4.2 \mathrm{~ms}$ for -180 $\mathrm{pA})$. With the current level of $-200 \mathrm{pA}$ or larger, the current duration that was needed to elicit a noticeable rebound was shorter and more constant, around $25 \mathrm{~ms}$, regardless of the level. To elicit a maximum rebound $(\sim 15-20 \mathrm{mV})$, with very small current, i.e., -40 pA, a very long (520.0 $\pm 25.6 \mathrm{~ms})$ current pulse was needed. As the current level was between $-60 \mathrm{pA}$ to $-140 \mathrm{pA}$, the duration of current pulse that was need to produce a maximum rebound was substantially reduced (from $195.0 \pm 9.7 \mathrm{~ms} \mathrm{~ms}$ to $120.0 \pm 7.8 \mathrm{~ms}$ ). With a current level of $-160 \mathrm{pA}$ or larger, the required current duration became rather 
constant, around $110.0 \pm 5.0 \mathrm{~ms}$, regardless of the level. These results indicate that there was a trade off between the duration and the level of hyperpolarization for generation of a rebound at lower current levels. When the hyperpolarizing current was larger than $\sim-160$ $\mathrm{pA}$, the required time of current injection for the maximum rebound was constant and there was no obvious trade off between the current level and duration. Anyhow, the data in the present study indicate that a hyperpolarizing current of as small as $-60 \mathrm{pA}$ (i.e., hyperpolarization of $-15 \mathrm{mV}$ to $-20 \mathrm{mV}$ ) for $100-200 \mathrm{~ms}$ is enough to generate a large rebound $(\sim 15 \mathrm{mV})$. 


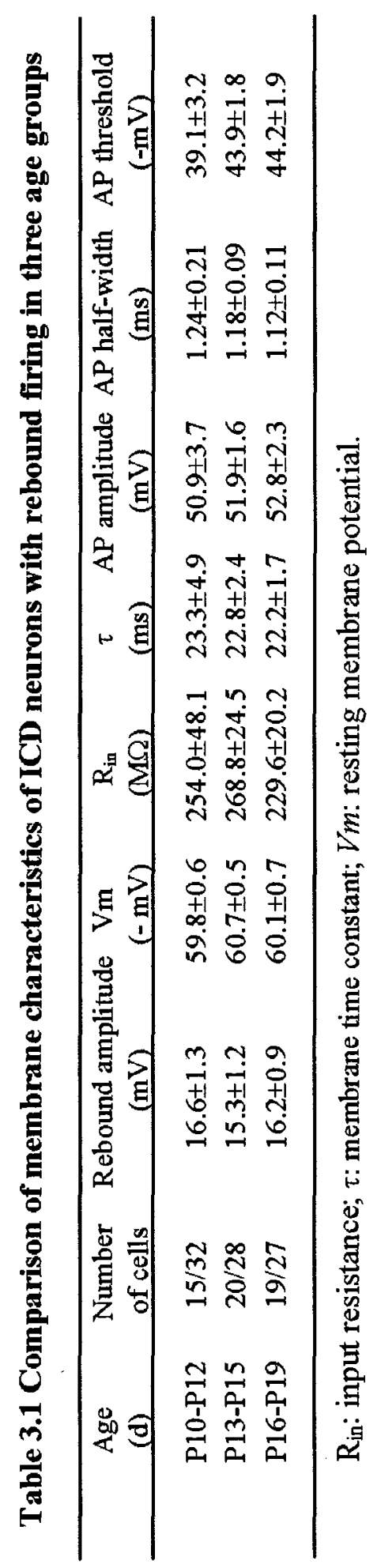



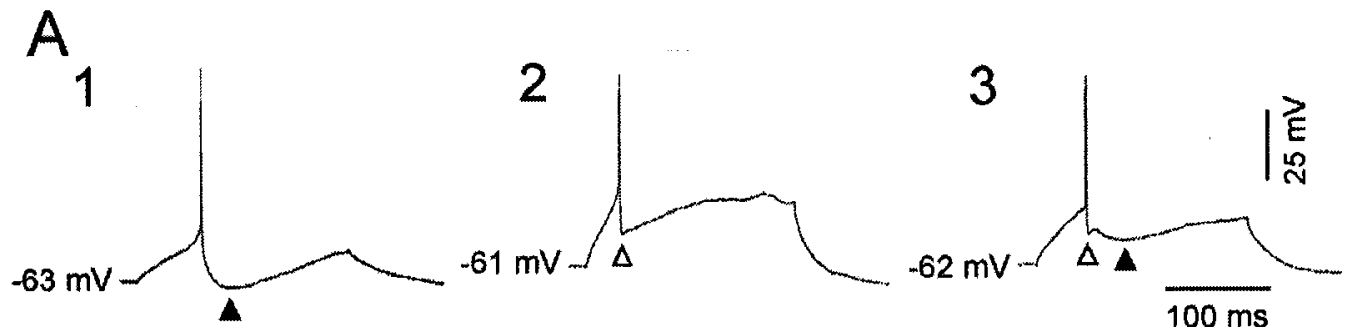

B

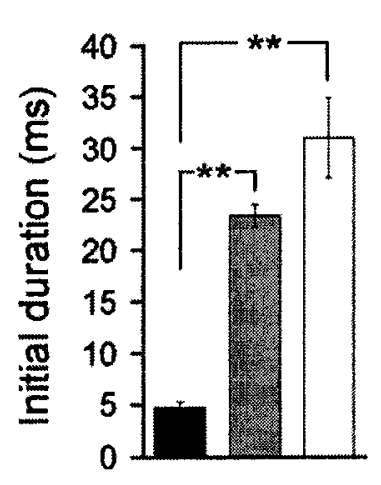

C

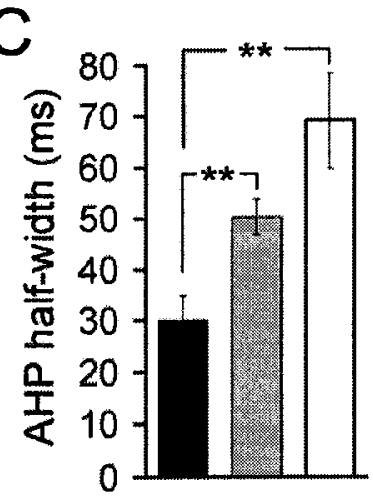

D

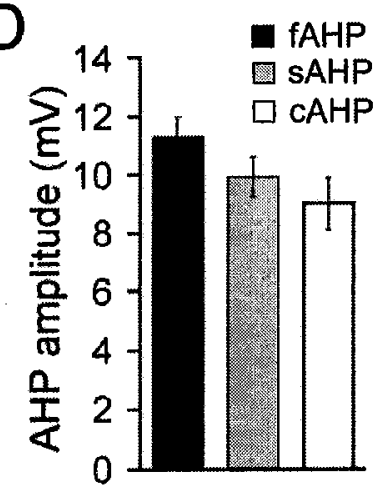

Figure 3.1. 
Figure 3.1. Characteristics of three types of AHP. $\mathbf{A}_{1}$. Slow AHP. $\mathbf{A}_{2}$. Fast AHP. $\mathbf{A}_{3}$. Combined AHP which consisted of fast (open triangle) and slow components (solid triangle). B. Comparisons of initial duration (from the AP peak to the lowest level of the AHP), half-width and amplitude of three types of AHP for 75 neurons. ${ }^{* *} \mathrm{p}<0.01$. 


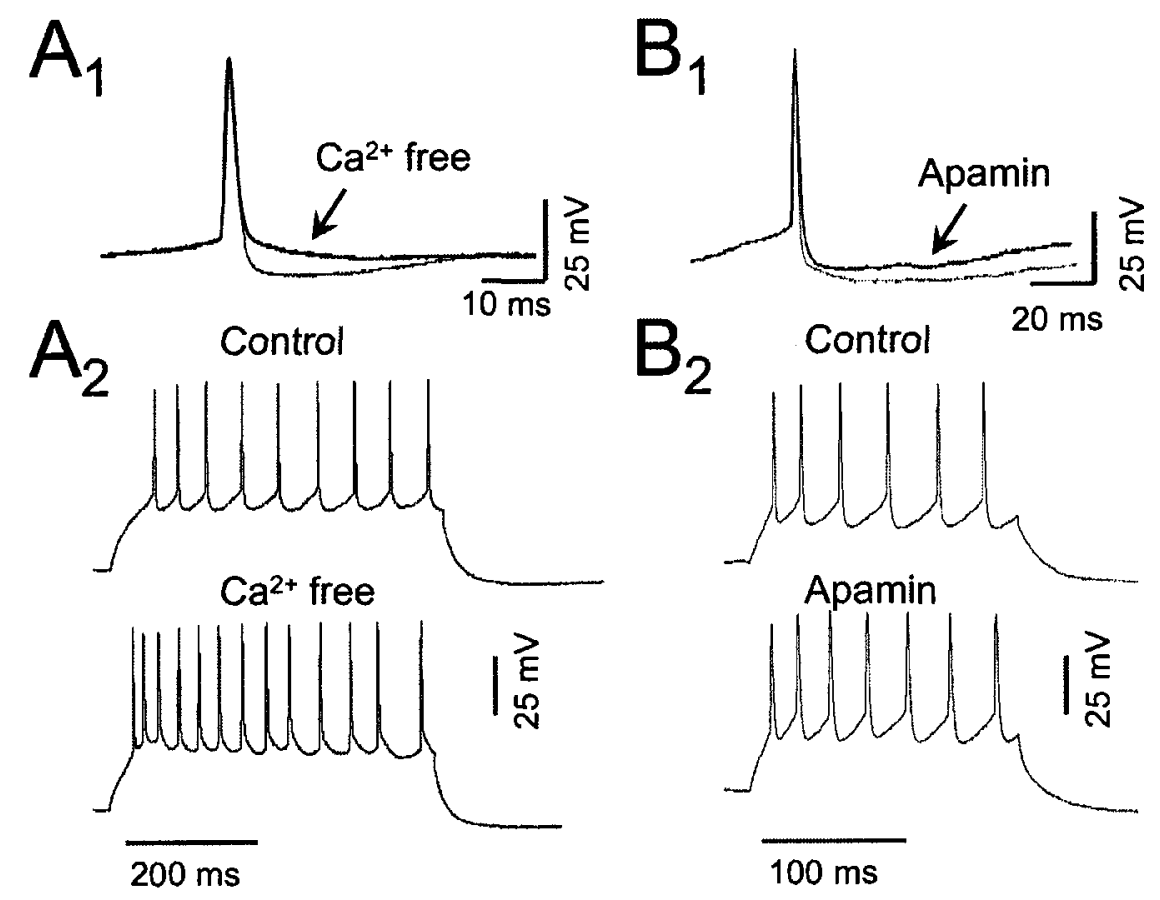

Figure 3.2. 
Figure 3.2. The AHP was mediated by $\mathrm{Ca}^{2+}$ activated $\mathrm{K}^{+}$current. $\mathbf{A}_{1}$. An AHP was abolished by removal of extracellular $\mathrm{Ca}^{2+}$. Arrow points to the AP trace that was recorded with the slice in $\mathrm{Ca}^{2+}$ free ACSF. $\mathbf{A}_{2}$. Responses to a positive current injection of $180 \mathrm{pA}$ with the slice in ACSF (upper trace) and $\mathrm{Ca}^{2+}$ free ACSF (lower trace). Note that the firing rate was increased after removal of extracellular $\mathrm{Ca}^{2+} \cdot \mathbf{B}_{1}$. An AHP was suppressed by apamin. $\mathbf{B}_{2}$. Responses to a positive current injection of $100 \mathrm{pA}$ with the slice in ACSF (upper trace) and ACSF containing apamin (lower trace). Note that the firing rate was slightly increased with application of apamin. 
$A_{1}$

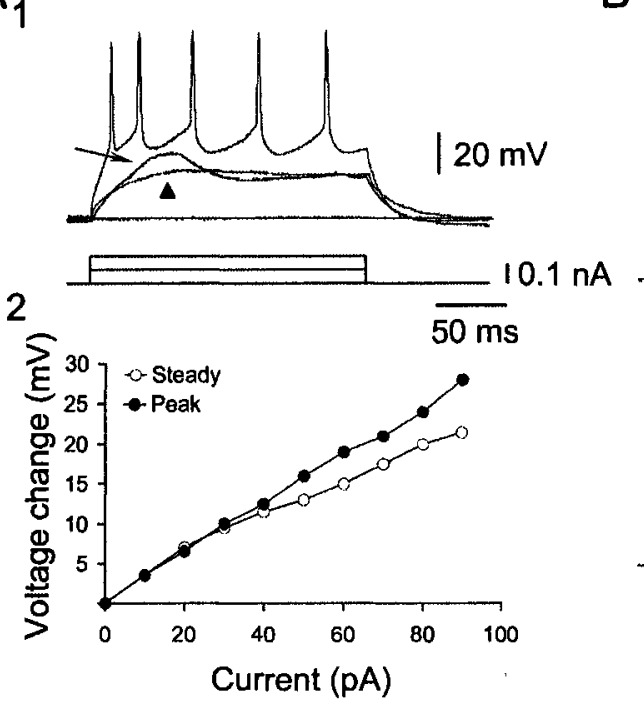

B

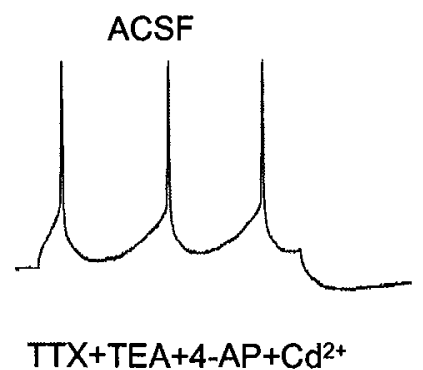
TTX+TEA+4-AP+Cd ${ }^{2+}$

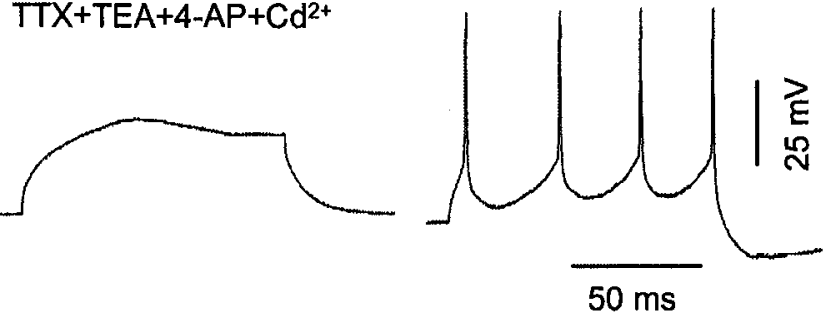

Figure 3.3 . 
Figure 3.3. The $\mathrm{Ca}^{2+}$ mediated hump and spikes. $\mathbf{A}_{1}$. Responses to current injections of $90 \mathrm{pA}$ (arrow) and $160 \mathrm{pA}$ (upper trace). Note a hump at the initial depolarization. With removal of extracellular $\mathrm{Ca}^{2+}$ the hump was eliminated (solid triangle). $\mathbf{A}_{2}$. The currentvoltage relationships acquired from voltage changes at the peak of the hump and the steady level of the depolarization. B. $\mathrm{Na}^{+}$spikes were elicited by a current injection of 70 pA in ACSF (left upper trace). $\mathrm{Ca}^{2+}$ spikes were revealed with a current of $150 \mathrm{pA}$ after blocking $\mathrm{Na}^{+}$and $\mathrm{K}^{+}$currents with TTX, TEA and 4-AP (right upper trace). These spikes were totally blocked by $\mathrm{CdCl}_{2}$ (left lower trace) $\mathrm{Na}^{+}$spikes were recovered after ACSF was reintroduced (right lower trace). 

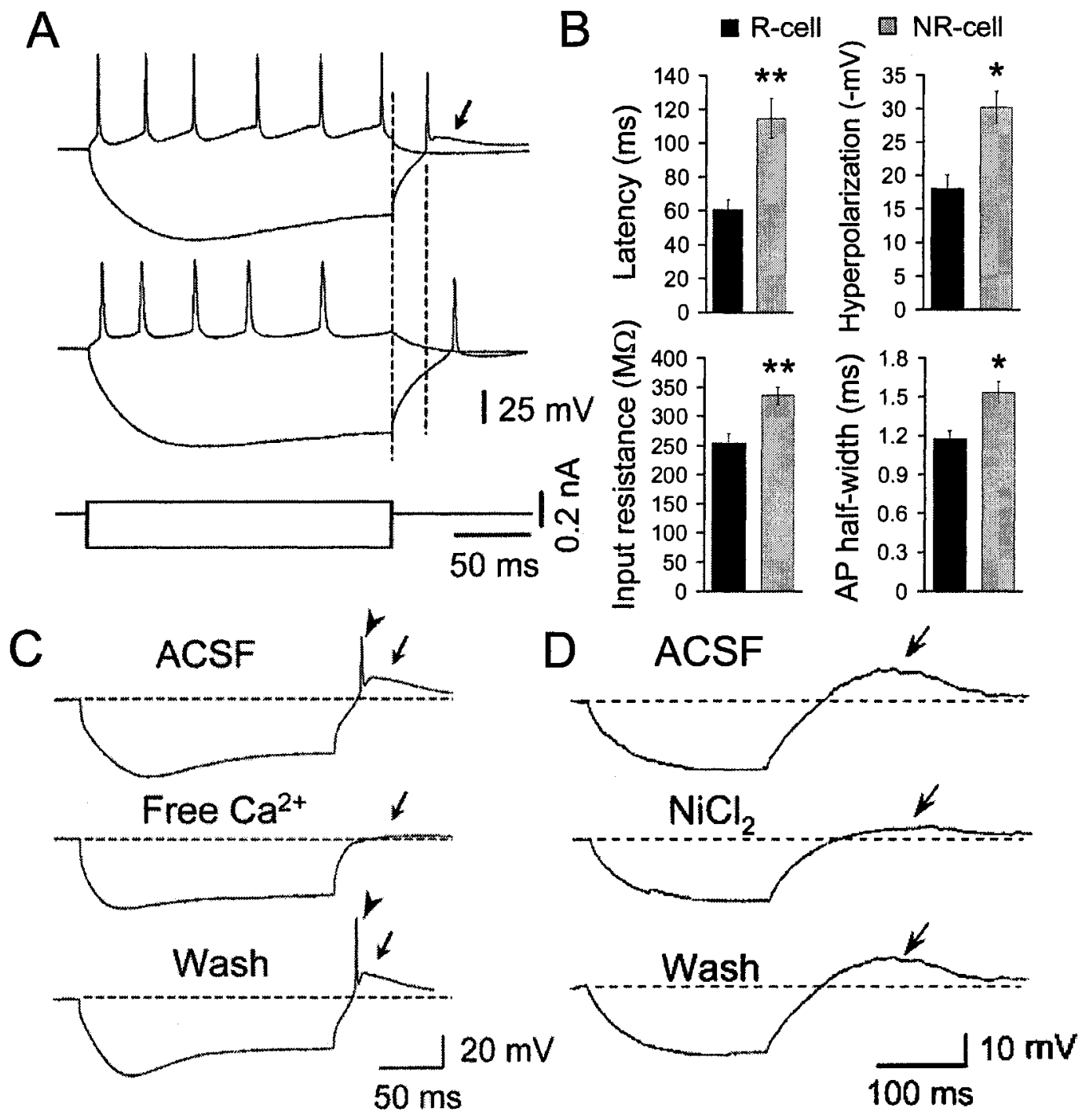

Figure 3.4 . 
Figure 3.4. The rebound was mediated by $\mathrm{Ca}^{2+}$ and comparisons of anode break spike in rebound and non-rebound neurons. A. Responses to positive (80 pA) and negative $(-200 \mathrm{pA})$ current injections in a rebound (upper traces) and a non-rebound ICD neuron (lower traces). Arrow points to the rebound. Note that a spike that arose from the rebound had a shorter latency than an anode break spike in the non-rebound neuron. $\mathbf{B}$. The average latency of the anode break spikes, hyperpolarization level for the spike, membrane input resistance and spike half-width for rebound (R) and non-rebound (NR) neurons were significantly different. ${ }^{* *} \mathrm{P}<0.01 .{ }^{*} \mathrm{P}<0.05$. The latency was measured at the threshold level of the anode break spikes. C. The rebound (arrow) and anode break spike (arrowhead) were blocked after removal of extracellular $\mathrm{Ca}^{2+} . \mathbf{D}$. The rebound was suppressed by $80 \%$ with an application of $\mathrm{NiCl}_{2}$. 


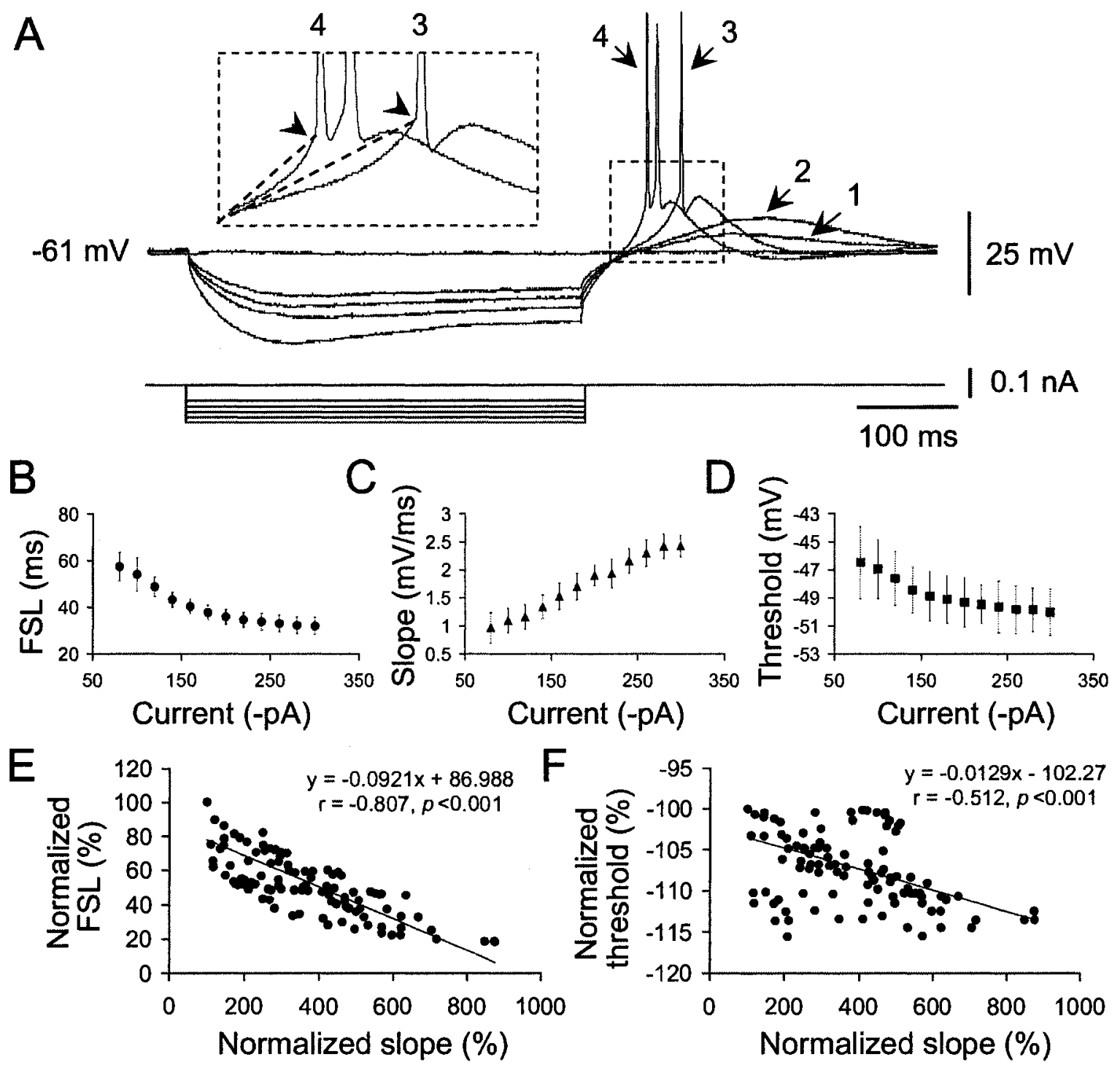

Figure 3.5. 
Figure 3.5. The latency and threshold of anode break spikes were related to the level of hyperpolarizing current and the slope of the rebound. A. Responses to negative current injections of $60,80,100$ and $140 \mathrm{pA}$ in a neuron. Note that the larger current injection led to a larger rebound and earlier anode break spikes (arrows 3 and 4). The larger rebound gave rise to an earlier anode break spike (insert, arrowheads 3 and 4). B. The average latency of the first spike was plotted as a function of current level. The latency decreased progressively as the current level was increased. $\mathbf{C}$. The average slope of the rebound was plotted as a function of current level. The slope increased progressively as the current level was increased. D. The average threshold of the spike was plotted as a function of current level. The threshold decreased progressively as the current level was increased. E and F. The relationships of normalized first spike latency and threshold to normalized slope of the rebound. Normalized first spike latency and threshold were negatively correlated with the normalized slope of the rebound $(p<0.001)$. The data were from 9 neurons. 


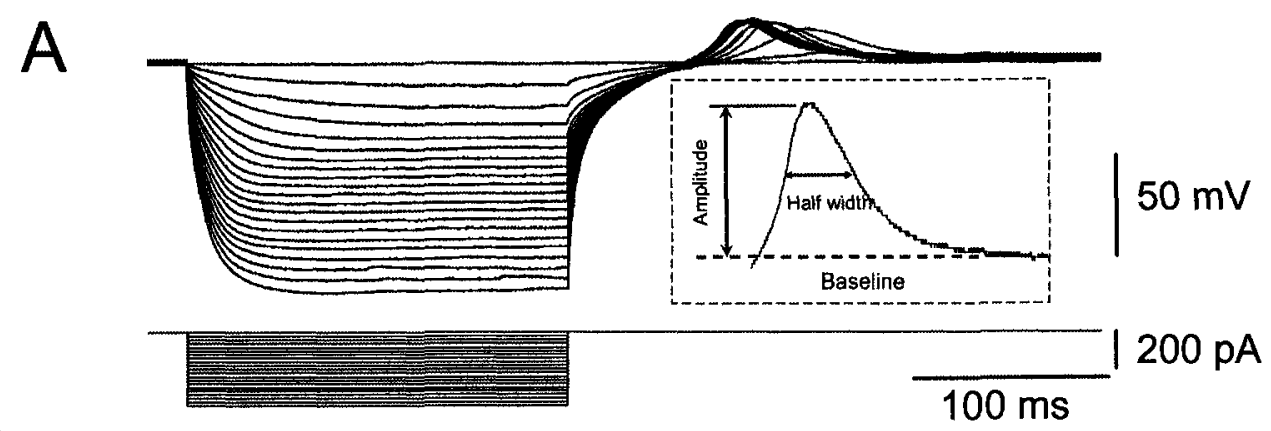

B
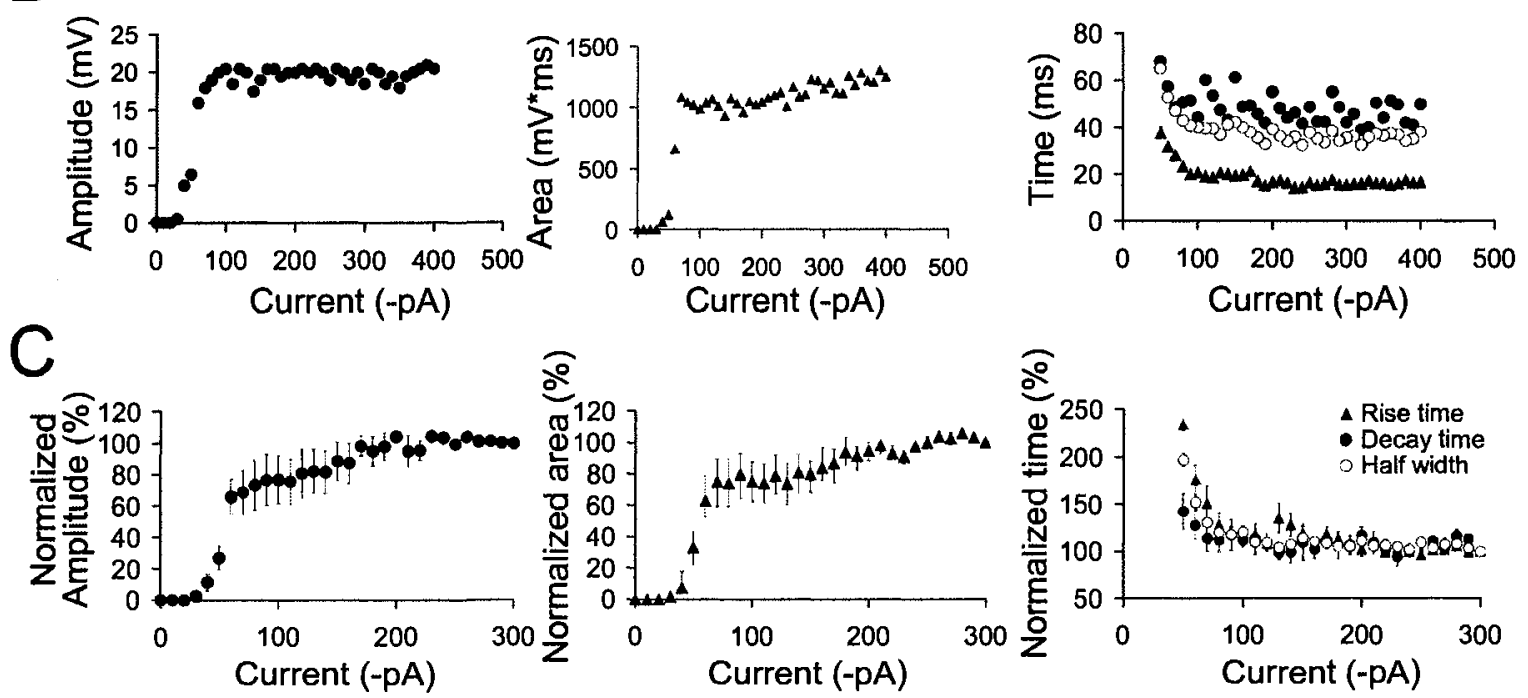

Figure 3.6. 
Figure 3.6. The relationships of the amplitude, area and kinetics of the rebound to the level of negative current injection. A. Responses to a series of negative current injections from 0 to $-400 \mathrm{pA}$ in $-20 \mathrm{pA} / \mathrm{step}$ for $200 \mathrm{~ms}$ in a neuron with the slice in ACSF containing TTX. B. The amplitude, area and kinetics (rise time, decay time and half width) of the rebound for the neuron shown in $\mathbf{A}$ were plotted as a function of the level of negative current. Note that the amplitude, area and kinetics became relatively constant with all levels of current injection larger than $-100 \mathrm{pA}$. C. The average normalized amplitude and area, and the kinetics of the rebound were plotted as a function of the level of current injection $(n=8)$. 


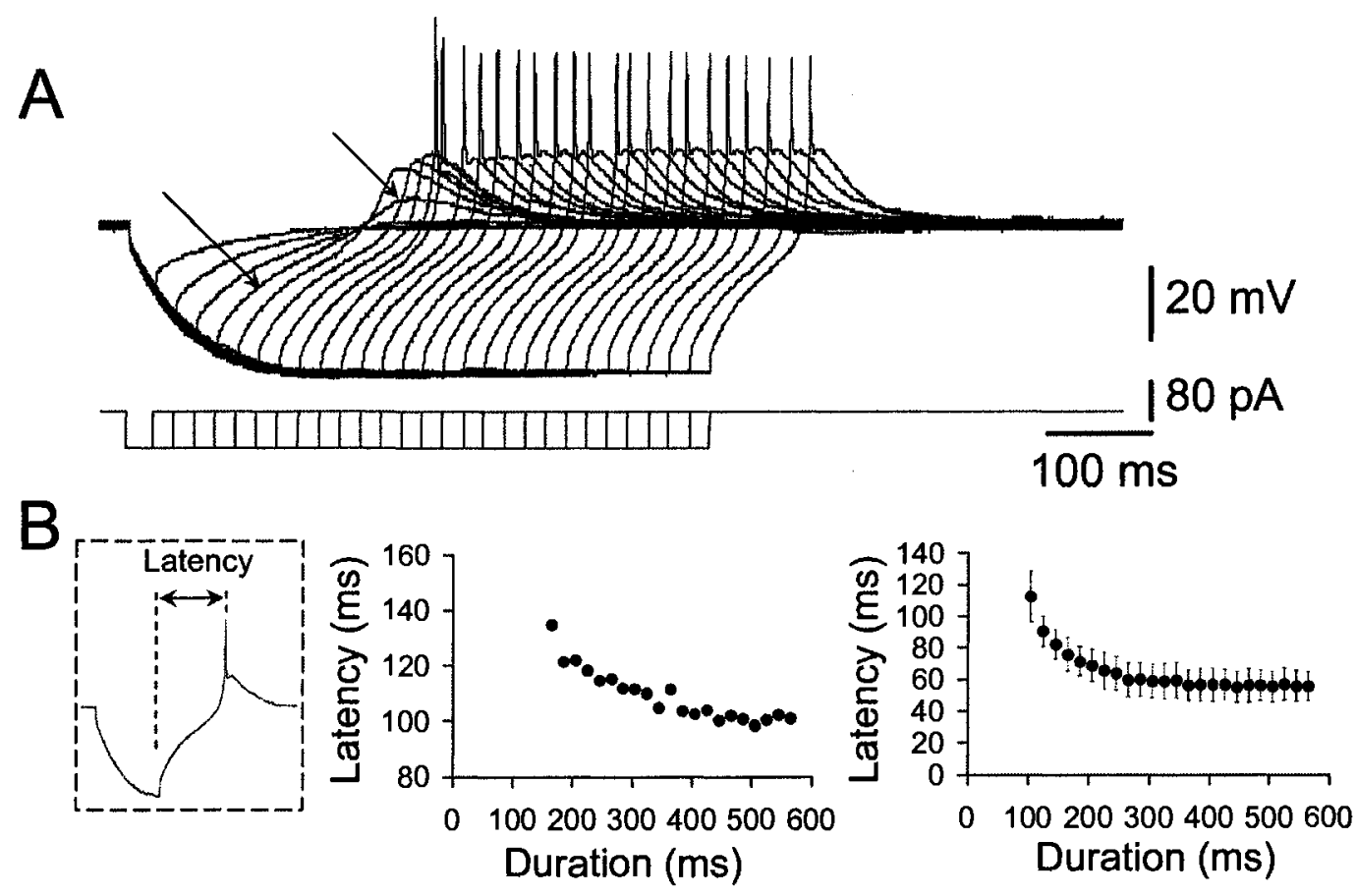

Figure 3.7. 
Figure 3.7. The relationship between the latency of anode break spikes and the duration of current injection. A. Responses to a series of negative current injections of $-80 \mathrm{pA}$ from $25-565 \mathrm{~ms}$ in $20 \mathrm{~ms} / \mathrm{step}$ in a neuron. Arrows indicate the response trace for the smallest rebound with $85 \mathrm{~ms}$ current injection. B. The spike latencies (measured as shown in the insert) were plotted as a function of the duration of current injection for the neuron shown in $\mathbf{A}$ (left panel) and for all neurons tested $(n=7)$ (right panel). Note that the spike occurred earlier from the rebound as the duration of current injection was lengthened. 
A
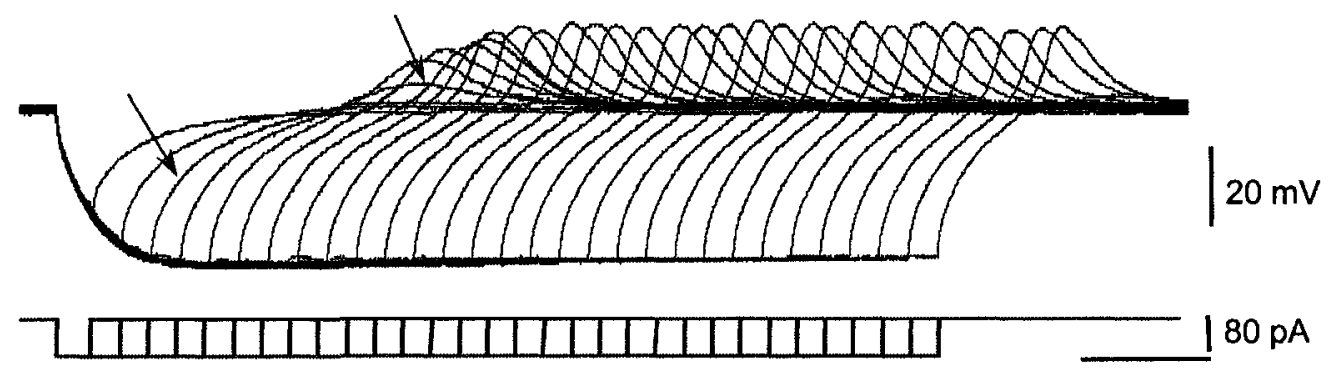

B
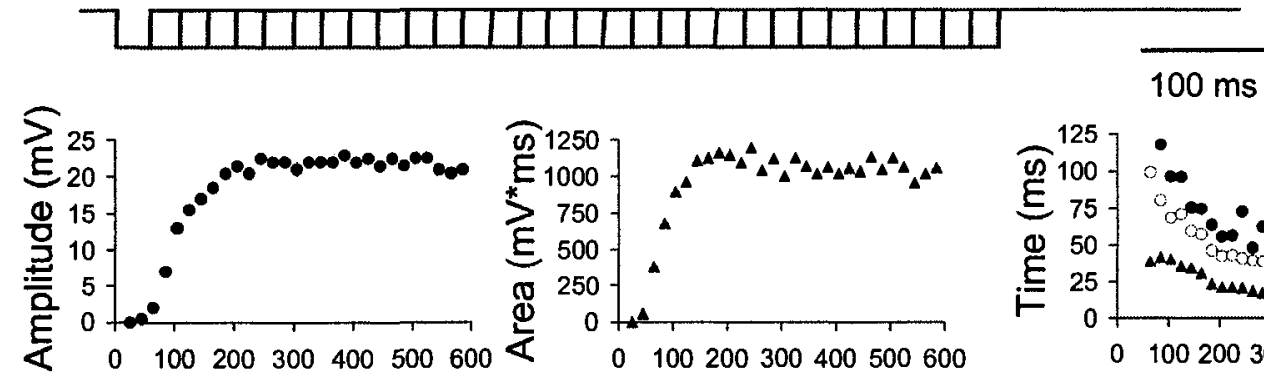

$80 \mathrm{pA}$

$\mathrm{C}$
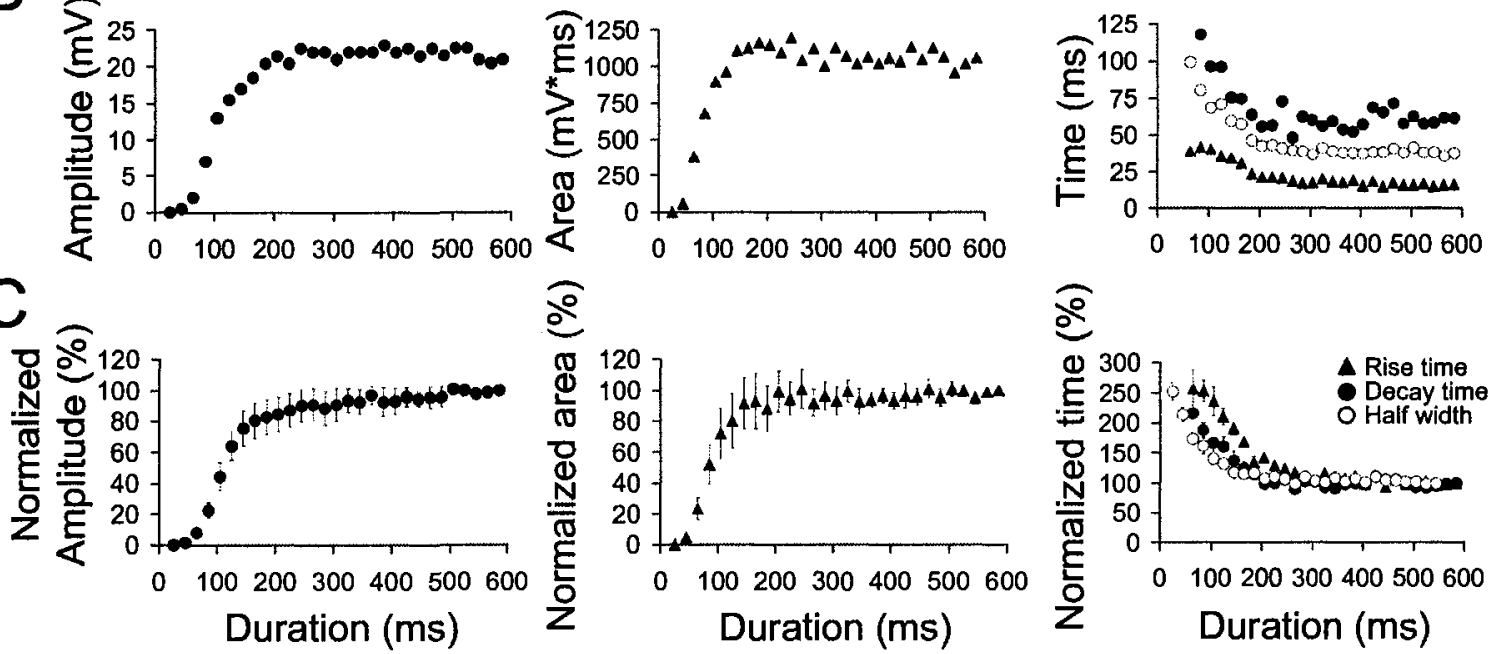

Figure 3.8. 
Figure 3.8. The relationships of the amplitude, area and kinetics of the rebound to the duration of negative current injection. A. Responses to a series of negative current injections of $-80 \mathrm{pA}$ from $25-585 \mathrm{~ms}$ in $20 \mathrm{~ms} / \mathrm{step}$ in a neuron. Arrows indicate the response trace for the smallest rebound with $65 \mathrm{~ms}$ current injection when the slice was in ACSF containing TTX. B. The amplitude, area and kinetics (rise time, decay time and half width) of the rebound for the neuron shown in $\mathbf{A}$ were plotted as a function of the duration of negative current. Note that the amplitude, area and kinetics became relatively constant with all durations of current injection longer than $200 \mathrm{~ms}$. C. The average normalized amplitude and area, and the kinetics of the rebound were plotted as a function of the duration of current injection $(n=6)$. 


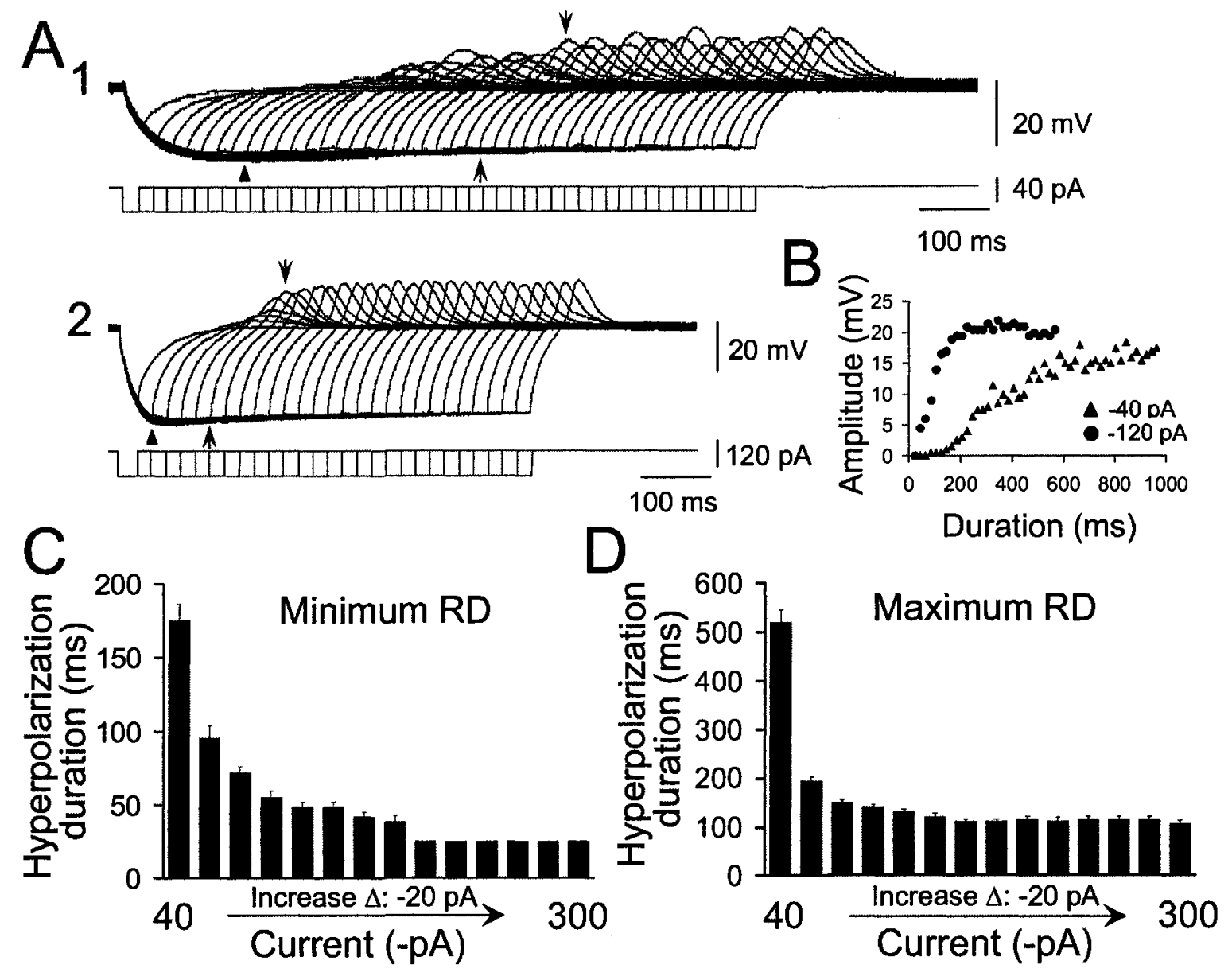

Figure 3.9. 
Figure 3.9. The level and duration of hyperpolarizing current required to induce minimum or maximum rebound. A. Response to a series of negative current of $-40 \mathrm{pA}$ (upper traces) from $25-925 \mathrm{~ms}$ and $-120 \mathrm{pA}$ (lower traces) from $25-565 \mathrm{~ms}$ in $20 \mathrm{~ms} / \mathrm{step}$ in a neurons. Arrowheads point to the traces for the minimum rebound and arrows point to the traces for the maximum rebound. B. The amplitude of the rebound was plotted as a function of the current duration for a level of $-40 \mathrm{pA}$ and $-120 \mathrm{pA}$ respectively. C. The level and duration of hyperpolarizing current required for inducing the minimum rebound $(n=6)$. D. The level and duration of hyperpolarizing current required for inducing the maximum rebound $(n=6)$. 


\subsection{Discussion}

The main results of this study showed that calcium contributes significantly to membrane excitability of ICD neurons. Action potentials of almost $80 \%$ of the neurons exhibited sAHP that was mediated by $\mathrm{Ca}^{2+}$-activated $\mathrm{K}^{+}$currents partially induced by activation of $\mathrm{SK}_{\mathrm{Ca}}$ channels. ICD neurons also generated $\mathrm{Ca}^{2+}$ spikes with membrane depolarization. In about half of the neurons a pronounced rebound depolarization was induced following membrane hyperpolarization. The rebound was mediated by $\mathrm{Ca}^{2+}$ currents that flowed primarily through low-threshold $\mathrm{T}$-type $\mathrm{Ca}^{2+}$ channels. The $\mathrm{Ca}^{2+}$ mediated rebound promoted generation of anode break action potentials. The anode break action potentials in rebound neurons were generated earlier with increases in level and duration of hyperpolarization. The threshold of the anode break spikes were decreased with the increases in level of hyperpolarization. There was a trade off between the magnitude and duration of hyperpolarization for generation of a rebound. The larger the hyperpolarization, the shorter the hyperpolarization required for generation of a rebound. However, when a neuron was injected with negative current more than $-160 \mathrm{pA}$ and longer than $110 \mathrm{~ms}$, the magnitude and kinetics of the rebound, including rise time, decay time and half-width, remained relatively constant with further increases in the level or duration of hyperpolarization.

\subsubsection{The role of $\mathrm{Ca}^{2+}$ in spiking}

In this study I classified AHP in ICD neurons as fAHP, sAHP and cAHP according to its time course and waveform. The fAHP was seen only in $\sim 15 \%$ of the neurons. The majority ICD neurons (78.7\%) had a sAHP, which lasted for around 100 
ms. The cAHP was seldom observed and consisted of both fast and slow components. The cAHP lasted for $\sim 100-200 \mathrm{~ms}$. The larger proportion of sAHP observed in ICD neurons is consistent with that found in neurons of the external cortex of IC (ICX) (Ahuja and $\mathrm{Wu}, 2007)$. Both SAHP and cAHP in this study may correspond to the medium AHP (mAHP) that lasts for several hundred milliseconds in hippocampal and neurons in other regions of the brain (Storm, 1989; Sah, 1996). I never observed an AHP that lasts for several seconds, which has been defined as SAHP by others (Storm, 1989). It has been demonstrated that although both mAHP and sAHP in those neurons are attributable to activation of $\mathrm{Ca}^{2+}$ activated $\mathrm{K}^{+}$currents, the sAHP is different in kinetics and also in pharmacology from the mAHP (Sah, 1996; Stocker et al., 1999; Stackman et al., 2002).

In this study removal of extracellular $\mathrm{Ca}^{2+}$ completely eliminated the sAHP, which confirms that the sAHP in ICD neurons is mediated by $\mathrm{Ca}^{2+}$ activated $\mathrm{K}^{+}$currents. The sAHP in ICD neurons was partially blocked by apamin, which has been demonstrated previously to block $\mathrm{SK}_{\mathrm{Ca}}$ ( $\mathrm{Li}$ and Bennett, 2007). These results are consistent with studies of neurons in the motor area of spinal cord, hippocampus, amygdala, subthalamus and cerebral cortex in which the mAHP is blocked by apamin (Stocker et al., 1999; Faber and Sah, 2002; Hallworth et al., 2003; Villalolbos et al., 2004; Teshima et al., 2003; Li and Bennette, 2007). The data suggest that the sAHP in ICD neurons may also be partially mediated by $\mathrm{SK}_{\mathrm{Ca}}$ current. On the other hand, the sAHP in ICD neurons was not completely blocked by apamin, therefore, some other $\mathrm{Ca}^{2+}$-activated $\mathrm{K}^{+}$conductance may be involved in generation of AHP. A study by Sivaramakrishnan and Oliver (2001) has shown that AHP in ICC neurons with an adapting firing pattern is blocked by charybdotoxin, the antagonist for $\mathrm{Ca}^{2+}$ activated $\mathrm{K}^{+}$ 
channels with large conductance $\left(\mathrm{BK}_{\mathrm{Ca}}\right)(\mathrm{Sah}, 1996$; Hille, 2001). There were no detailed analyses of the kinetics of the AHP in those ICC neurons. Therefore, I can't compare the time course and types of the AHP in neurons of ICC and ICD. Nevertheless, AHP in ICD neurons may be mediated by more than one type of $\mathrm{Ca}^{2+}$ activated $\mathrm{K}^{+}$conductance. Further investigation is required to clarify whether the SAHP in ICD neurons is also mediated by $\mathrm{BK}_{\mathrm{Ca}}$ or some other types of currents.

In some ICD neurons depolarizing current injection elicited a broad hump which was eliminated by removal of extracellular $\mathrm{Ca}^{2+}$, indicating that the hump is $\mathrm{Ca}^{2+}$ mediated. Such a hump has also been reported by Smith (1992), Li et al. (1998) and Ahuja and Wu (2007). In Li et al's study (1998) the hump was revealed by removal of extracellular $\mathrm{Na}^{+}$, and was seen more commonly in ICD than ICX neurons, but not in ICC neurons. In the study of ICC neurons by Sivaramakrishnan and Oliver (2001), a $\mathrm{Ca}^{2+}$ mediated hump was not reported. Smith (1992) observed a $\mathrm{Ca}^{2+}$ mediated hump in IC cortex neurons whose location was not determined. In Ahuja and Wu's study (2007), the hump was revealed in ICX neurons only after blocking $\mathrm{Na}^{+}$and $\mathrm{K}^{+}$currents. In the present study the $\mathrm{Ca}^{2+}$ mediated hump was observed in more than $30 \%$ of the neurons without any manipulation of extracellular ions or ion channel antagonists. Various types of voltage-gated $\mathrm{Ca}^{2+}$ channels have been identified in the IC. These channels include low threshold voltage-activated (LVA) and high threshold voltage-activated (HVA) $\mathrm{Ca}^{2+}$ currents (N'gouemo and Rittenhouse, 2000; N'gouemo and Morad, 2003). The LVA current was activated at around $-60 \mathrm{mV}$ and was expressed in around $30 \%$ IC neurons, whereas HVA current activated at around $-40 \mathrm{mV}$ and was recorded in all IC neurons (N'gouemo and Rittenhouse, 2000; N'gouemo and Morad, 2003). Generation of the $\mathrm{Ca}^{2+}$ 
mediated hump in ICD neurons is likely due to activation of $\mathrm{HVA} \mathrm{Ca}^{2+}$ current since the hump was elicited by depolarization about $20 \mathrm{mV}$ positive to the resting potential. Furthermore, the hump was more often seen in neurons of ICD than other IC subdivisions, suggesting that ICD expresses more HVA $\mathrm{Ca}^{2+}$ channels than ICX and ICC. In addition to the hump, $\mathrm{Ca}^{2+}$ mediated spikes were revealed in ICD neurons after blocking $\mathrm{Na}^{+}$and $\mathrm{K}^{+}$currents in the present study. The results are consistent with those from other studies of ICC and IC cortex neurons (Smith, 1992; Li et al., 1998; Sivaramakrishnan and Oliver, 2001; Ahuja and Wu, 2007). $\mathrm{HVA} \mathrm{Ca}^{2+}$ currents may account for $\mathrm{Ca}^{2+}$ spikes in ICD neurons as a higher level of depolarizing current was required to elicit $\mathrm{Ca}^{2+}$ spikes compared to that for $\mathrm{Na}^{+}$spikes, which is consistent with the fact that the threshold for $\mathrm{Ca}^{2+}$ spikes was higher than those for $\mathrm{Na}^{+}$spikes (Viana et al., 1993).

\subsubsection{Contribution of $\mathrm{Ca}^{2+}$ mediated rebound to membrane excitability}

A rebound depolarization in ICD neurons was generated upon release of membrane hyperpolarization. One or several spikes were often elicited from the rebound. The rebound in ICD neurons was completely eliminated by removal of extracellular $\mathrm{Ca}^{2+}$, but not by application of TTX. These results suggest that the rebound is mediated by $\mathrm{Ca}^{2+}$ current. The $\mathrm{Ca}^{2+}$ mediated rebound has been demonstrated in other neurons, including ICC and IC cortex neurons (Smith, 1992; Viana et al., 1993; Beurrier et al., 1999; Fan et al., 2000; Sivaramakrishnan and Oliver, 2001; Surge et al., 2006). The physiological characteristics of the rebound in ICD neurons are also consistent with the notion that the rebound is mediated primarily by activation of low-threshold $\mathrm{T}$-type $\mathrm{Ca}^{2+}$ channels. First, 
the membrane potential at which the rebound was elicited is near the neuron's resting potential, i.e., $\sim-60 \mathrm{mV}$, which is consistent with the activation range of T-type $\mathrm{Ca}^{2+}$ channels in IC neurons (N'Gouemo and Rittenhouse, 2000; N'Gouemo and Morad, 2003). Second, the rebound occurred always after hyperpolarization, which is consistent with deinactivation of $\mathrm{T}$-type $\mathrm{Ca}^{2+}$ channels due to the recovery of channels from inactivation (Perez-Reyes, 2003). Third, the increase in magnitude and time of the hyperpolarizing pulse led to a larger rebound, which is consistent with the kinetics of Ttype $\mathrm{Ca}^{2+}$ channels (Carbone and Lux, 1984; Viana et al., 1993; Alexander et al., 2006). Fourth, the rebound is suppressed by $\mathrm{Ni}^{2+}$ which has been shown to block low-threshold T-type $\mathrm{Ca}^{2+}$ channels (Ryu and Randic, 1990; Fan et al., 2000).

N'Gouemo and Rittenhouse (2000) and N'Gouemo and Morad (2003) have recorded $\mathrm{LVA} \mathrm{Ca}^{2+}$ currents, i.e., T-type, in around $1 / 3$ of the IC neurons of neonatal and adult rats. In the study of ICC by Sivaramakrishnan and Oliver (2001) and the present study, the proportion of rebound neurons was very similar in ICC and ICD, i.e., slightly more than half of the sampled neurons. As the rebound correlates with activation of lowthreshold T-type $\mathrm{Ca}^{2+}$ channels, it seems that at least half of ICC and ICD neurons express this type of $\mathrm{Ca}^{2+}$ channels, suggesting a prevalence of low voltage T-type $\mathrm{Ca}^{2+}$ channels in the IC. ICD neurons respond to depolarizing current injection with three firing patterns, i.e., sustained-regular, sustained adapting or buildup (Sun and Wu, 2007). The rebound is observed only in neurons with sustained firing, but not in neurons with a buildup firing pattern with which the firing starts after a long delay and the membrane potential gradually builds up toward the threshold of action potentials during the delay. 
These results together with the results of this study suggest that low-threshold T-type $\mathrm{Ca}^{2+}$ channels are expressed only in ICD neurons with sustained firing.

One of the well-known properties of T-type $\mathrm{Ca}^{2+}$ channels is that they rapidly inactivate in a time- and voltage-dependent manner. Hyperpolarization is necessary to remove the inactivation, which then makes the channel activation possible. Thus, I can predict that increasing amplitude of hyperpolarization would remove more inactivation and elicit a stronger rebound depolarization after the membrane returns to the resting level. This was indeed the case for the neurons in the present study. Furthermore, after block of anode break spikes with TTX, I found that the magnitude, including the amplitude and area, and kinetics of the rebound were dependent on the level (up to $\sim-100$ pA) and duration (up to $\sim 200 \mathrm{~ms}$ ) of the hyperpolarization. A larger and longer hyperpolarization induced a larger rebound. These results are consistent with those of previous studies of neurons in cerebellum, thalamus and subthalamus (Aizenman \& Linden, 1999; Beurrier et al., 1999; Alexander et al., 2006). Neurons in these regions have bursting spikes on the rebound. The number of bursting spikes was used as an index of the rebound strength. For example, with a hyperpolarizing current pulse of -100 to $500 \mathrm{pA}$ for $250 \mathrm{~ms}$ or $-500 \mathrm{pA}$ for 50 to $500 \mathrm{~ms}$, the number of spikes increased as either the level or duration of hyperpolarization was increased (Aizenman and Linden, 1999). After the anode break spikes were blocked, the amplitude and area of the rebound were also further increased as the level or duration of hyperpolarization was increased. (Aizenman and Linden, 1999).

Furthermore, there was a trade off between the magnitude of hyperpolarization and its duration for generation of the rebound. In other words, the rebound can be 
induced either by a larger and shorter hyperpolarization or a smaller or longer hyperpolarization (Fig. 3.9). The time- and voltage-dependent process of recovery from inactivation controls the return of availability of channels subsequent to inactivation. These results are consistent with time- and voltage-dependent process of deinactivation (or recovery) of T-type $\mathrm{Ca}^{2+}$ channels (Huguenard and Prince, 1992). The degree of deinactivation of T-type $\mathrm{Ca}^{2+}$ channels is governed by the magnitude and duration of the hyperpolarization. In other words, a larger or longer hyperpolarization would cause more deinactivation of $\mathrm{T}$-type $\mathrm{Ca}^{2+}$ channels, and, therefore, a larger rebound. Once inactivation of the channels is largely removed by a sufficiently large or long hyperpolarization, a maximum activation of the channels can be achieved when the neuron returns back to its resting potential, therefore no further increase in the rebound would be produced by additional hyperpolarization. Anyhow, the data showed that a hyperpolarization of as small as $\sim-15$ to $-20 \mathrm{mV}$ for $100-200 \mathrm{~ms}$ is enough to generate a large rebound $(\sim 15 \mathrm{mV})$. The rebound with this level would be sufficient for producing anode break spikes or summating subthreshold synaptic excitation for generating spikes.

When a rebound was sufficiently large, anode break spikes were often elicited. The threshold and latency of the spike were closely related to the level and duration of the hyperpolarization. A larger hyperpolarization usually gave rise to an anode break spike with a shorter latency and lower threshold. I also found a negative correlation of the spike latency and threshold with the slope of the rebound. These results are consistent with the kinetics of deinactivation of T-type $\mathrm{Ca}^{2+}$ channels. A large hyperpolarization quickly removes inactivation of T-type $\mathrm{Ca}^{2+}$ channels and rapidly activates $\mathrm{T}$-type $\mathrm{Ca}^{2+}$ channels, thus producing a rebound with a fast rise time. A rebound with a large slope 
would facilitate generation of $\mathrm{Na}^{+}$spikes, thus lowering the spike threshold and shortening the latency.

\subsubsection{Functional implications}

The results in the present study have shown that $\mathrm{Ca}^{2+}$ contributes to membrane excitability of ICD neurons in several ways: by causing hyperpolarization (i.e., AHP) through opening of $\mathrm{Ca}^{2+}$ activated $\mathrm{K}^{+}$channels, i.e., partially $\mathrm{SK}_{\mathrm{Ca}}$; by causing depolarization (or spikes) through direct influx of $\mathrm{Ca}^{2+}$ probably into high-threshold $\mathrm{Ca}^{2+}$ channels and by causing rebound depolarization after membrane hyperpolarization through activation of low-threshold T-type $\mathrm{Ca}^{2+}$ channels. The AHP regulates firing rate of ICD neurons as removal of extracellular $\mathrm{Ca}^{2+}$ or suppression of $\mathrm{SK}_{\mathrm{Ca}}$ channels led to an increase in firing rate. $\mathrm{Ca}^{2+}$ promotes activation of voltage-gated $\mathrm{Na}^{+}$channels for generation of action potentials in ICD neurons as the $\mathrm{Ca}^{2+}$ mediated hump could be elicited at a membrane potential below the threshold for $\mathrm{Na}^{+}$spikes.

$\mathrm{Ca}^{2+}$ mediated rebound depolarization can modulate membrane excitability of ICD neurons after the neuron is hyperpolarized. During the rebound not only was the membrane potential depolarized, but also action potentials were more likely generated and occurred earlier than anode break spikes in non-rebound neurons. Thus the rebound seems to enhance membrane excitability immediately after the neuron is inhibited.

The rebound depolarization may be directly responsible for "off" responses recorded from auditory brainstem neurons in vivo (Kuwada and Batra, 1999). The neurons with "off" responses can encode the pattern of amplitude-modulated sounds with higher synchrony than neurons with sustained discharge responses during the sound. 
These results are consistent with the results from the present study. Once the hyperpolarization was sufficiently large or long, the latency of the $1^{\text {st }}$ anode break spike on the rebound became relatively constant with increase in strength or duration of the hyperpolarization (Fig. 3.5 and 3.7). Both in vivo and in vitro results suggest that the rebound mechanism allows the neuron to encode ongoing envelopes of a sound precisely (Kuwada and Batra, 1999).

The rebound may also be a neuronal mechanism for summating an excitatory input that arrives simultaneously with the rebound. Thus, even if the rebound is subthreshold, as a result of summation, the neuron would more likely to produce spikes. This principle has been used to explain tuning to sound duration, frequency-modulated sweep direction, the rate of periodic frequency or amplitude modulations and sound gaps in IC neurons (Casseday et al., 1994; Ehrlich et al., 1997; Covey and Casseday, 1999; Brand et al., 2000; Large and Crawford, 2002; Faure et al., 2003; Galazyuk et al., 2005).

Generation of the rebound requires a preceding membrane hyperpolarization. The data from the present study indicate that a hyperpolarization as small as $\sim-15 \mathrm{mV}$ for less than $100 \mathrm{~ms}$ could induce a minimum rebound, and for 100-200 ms typically induce a maximum rebound. Anatomical studies have shown that the ICD receives inhibitory inputs which may come from either contralateral IC or ipsilateral ICC (Oliver, 2005; Saldaña and Merchán, 2005). The auditory cortex also sends inhibitory projections to the ICD (Winer, 2005). These inhibitory inputs are likely GABAergic and would evoke postsynaptic responses (IPSPs) through either $\mathrm{GABA}_{A}$ or $\mathrm{GABA}_{B}$ receptors (Kelly and Caspary, 2005; Sun and $\mathrm{Wu}, 2006$ ). The amplitude of a $\mathrm{GABA}_{\mathrm{A}}$ receptor mediated IPSP elicited by a single electrical shock to the afferent inputs in auditory brainstem neurons, 
including IC neurons, can be as large as $-10 \mathrm{mV}$ to $-15 \mathrm{mV}$ and the time course can be as long as $100 \mathrm{~ms}$ (Vale et al., 2000; Vale and Sanes, 2003; Bevan et al., 2002; Wu et al., 2004; Irfan et al., 2005). Much larger and longer $\mathrm{GABA}_{\mathrm{A}}$ receptor mediated IPSPs can also be evoked by repetitive stimulation of the synaptic inputs (Bevan et al., 2002; Wu et al., 2004). These IPSPs would hyperpolarize a neuron sufficiently to produce a rebound depolarization (Bevan et al., 2002). ICD neurons have been demonstrated to express $\mathrm{GABA}_{\mathrm{B}}$ receptors (Fubara et al., 1996). Repetitive electrical stimulation of the commissure of the IC evoked $\mathrm{GABA}_{\mathrm{B}}$ receptor mediated inhibitory synaptic response that can last for more than $500 \mathrm{~ms}$ (Sun and Wu, 2006). All these data indicate that both $\mathrm{GABA}_{A}$ and $\mathrm{GABA}_{B}$ receptor mediated inhibition in ICD neurons can meet the requirement for strength and duration of hyperpolarization to induce a substantial rebound depolarization. Thus, the rebound depolarization after synaptic inhibition would provide a powerful mechanism to IC neurons for encoding specific attributes of acoustic information. 


\section{Chapter 4}

\section{Physiological roles of $G A B A_{B}$ receptors in neurons of the dorsal cortex of the inferior colliculus}

\subsection{Introduction}

Inhibition plays a prominent role in determining the output of auditory neurons in response to acoustic stimulation (Nataraj and Wenstrup, 2005). In the IC, GABA is the major inhibitory neurotransmitter (Faingold, 2002) which is important in regulating physiological responses of IC neurons. The GABAergic system has at least two strategies to enrich the action of GABA. First, GABA is released at different subcellular locations and precisely defined time windows during circuit operation. Second, different physiological effects of GABA are mediated by different GABA receptors, such as ionotropic $G_{A B A}$ receptors and metabotropic $G_{A B A}$ receptors. $G_{A B A}$ receptors mediate fast GABAergic inhibition (Faingold, 2002). Activation of presynaptic GABA receptors located on GABAergic terminals or other terminals can inhibit transmitter release, while activation of postsynaptic $\mathrm{GABA}_{B}$ receptors mediates prolonged neuronal hyperpolarization (Bowery, 2006). Through $\mathrm{GABA}_{\mathrm{A}}$ receptors, GABAergic inhibition can decrease the discharge rate of almost all neurons that are responsive to sound in the IC (Faingold et al., 1991; Li and Kelly, 1992; Kelly and Caspary, 2005). GABAergic inhibition also contributes to shaping binaural responses (Faingold et al., 1991; Fujita and Konishi, 1991; Park and Pollak, 1993; Kelly and Caspary, 2005), to directional selectivity to frequency modulated sweeps (Fuzessery and Hall, 1996; Kelly and Caspary, 2005; Nataraj and Wenstrup, 2005), to duration tuning (Casseday et al., 1994; Kelly and Caspary, 2005; Nataraj and Wenstrup, 2005), to the complex frequency responses 
(LeBeau et al., 2001; Nataraj and Wenstrup, 2005), and to the coding of ITD of low frequency sounds (D’Angelo et al., 2005).

The physiological role of $\mathrm{GABA}_{\mathrm{B}}$ receptor mediated inhibition in auditory function remains unclear. In the ICC, in vivo studies have demonstrated that baclofen, a $\mathrm{GABA}_{\mathrm{B}}$ receptor agonist, applied iontophoretically or systemically reduced soundevoked responses, while phaclofen or CGP35348, the $\mathrm{GABA}_{\mathrm{B}}$ receptors antagonists, increased the responses to tone and sinusoidally amplitude modulated sounds (Faingold et al., 1991; Szczepaniak and Moller, 1996; Vaughn et al., 1996; Burger and Pollak, 1998). However, all these studies were unable to determine the action of $\mathrm{GABA}_{\mathrm{B}}$ receptor at pre- and/or postsynaptic sites. Previous in vitro studies by Ma et al., (2002) and Sun et al., (2006) have provided the evidence that activation of presynaptic GABA receptors depressed the GABAergic inhibition and glutamatergic excitation in ICC neurons. In addition, $\mathrm{GABA}_{\mathrm{B}}$ receptor agonist, baclofen, did not affect the cell resting membrane conductance and the firing rate in response to depolarizing current in ICC neurons (Ma et al., 2002). Therefore, little evidence supports the presence of postsynaptic $\mathrm{GABA}_{\mathrm{B}}$ receptors in ICC neurons.

In contrast to the ICC, a striking feature of the ICD is the strong expression of $\mathrm{GABA}_{\mathrm{B}}$ receptors (Milbrandt et al., 1994; Fubara et al., 1996). Therefore, it is likely that both pre- and postsynaptic $\mathrm{GABA}_{\mathrm{B}}$ receptors are involved in $\mathrm{GABA}_{\mathrm{B}}$-induced inhibition. The main goal of the present study is to investigate functional contribution of pre- and postsynaptic $\mathrm{GABA}_{\mathrm{B}}$ receptors to the membrane excitability and synaptic transmission in ICD neurons. The present observations indicate the presence of both pre- and postsynaptic $\mathrm{GABA}_{\mathrm{B}}$ receptors in ICD neurons. Activation of postsynaptic $\mathrm{GABA}_{\mathrm{B}}$ 
receptors modulated the membrane excitability of ICD neurons through the activity of several distinct ion channels. Activation of presynaptic $\mathrm{GABA}_{\mathrm{B}}$ receptors induced depression of neurotransmitter release including both GABA and glutamate. Therefore, functional $\mathrm{GABA}_{\mathrm{B}}$ receptors in the ICD provide a mechanism for setting an appropriate level of excitation and inhibition, which is critical for auditory processing.

\subsection{Materials and Methods}

\subsubsection{Preparation of brain slices}

Same as that described in Chapter 2.

\subsubsection{Whole-cell patch clamp recording}

Electrodes for whole-cell patch clamp recordings were constructed from thinwalled glass pipettes (Kimax-51; Kimble, Vineland, NJ) with a two-stage vertical puller (PP830, Narishige, Tokyo, Japan). Unless otherwise stated, the electrode was filled with an internal solution containing (in $\mathrm{mM}$ ): $130 \mathrm{~K}$-gluconate, 0.6 EGTA, 10 HEPES, 2 $\mathrm{MgCl}_{2}, 5 \mathrm{KCl}, 2 \mathrm{ATP}$, and $0.3 \mathrm{GTP}$. The $\mathrm{pH}$ of the solution was adjusted to 7.25 with $\mathrm{KOH}$ and the osmolarity was $280-290 \mathrm{mOsm} / \mathrm{kg}$. In experiments where the function of presynaptic $\mathrm{GABA}_{\mathrm{B}}$ receptors was investigated, the patch recording pipettes were filled with a solution containing the following (in $\mathrm{mM}$ ): $110 \mathrm{CsF}, 20$ tetraethylammonium (TEA), 10 EGTA, 10 HEPES, $2 \mathrm{MgCl}_{2}, 4 \mathrm{NaCl}, 2$ ATP, $0.5 \mathrm{QX}-314\left(\mathrm{C}_{16} \mathrm{H}_{27} \mathrm{BrN}_{2} \mathrm{O}\right)$. The $\mathrm{pH}$ of the solution was $7.2-7.3$ and the osmolarity was $280 \mathrm{mOsm} / \mathrm{kg}$. The resistance of the patch electrode was 4-7 M . Whole-cell patch clamp recordings were made by an EPC-8 patch-clamp amplifier (HEKA, Darmstadt, Germany) or Axopatch 200A Amplifier (Axon Instruments). ICD neurons were visualized under a Zeiss Axioskop microscope by a $40 \mathrm{X}$ water immersion objective with Hoffman modification contrast. 
The membrane potentials reported in this study were corrected with the junction potential. Series resistances were in the range of $15-25 \mathrm{M} \Omega$ and were compensated by $50-70 \%$. If the series resistance changed by more than $15 \%$ of the initial value during the recording, the data were discarded. To separate two distinct components of voltage-activated $\mathrm{K}^{+}$ currents, fast inactivating transient current $I K_{A}$ and sustained current $I K_{s}$, the following steps modified from the protocol used in a previous study (Sivaramakrishnan and Oliver, 2001) were applied. The neurons was held at $-60 \mathrm{mV}$ and then stepped to either $-100 \mathrm{mV}$ (protocol A) or $-40 \mathrm{mV}$ (protocol B) for $300 \mathrm{~ms}$ (conditioning prepulse potential). Then the membrane was depolarized from $-60 \mathrm{mV}$ to $40 \mathrm{mV}$ in increments of $10 \mathrm{mV}$ of each step for $300 \mathrm{~ms}$. Voltage-activated $\mathrm{K}^{+}$currents including both $I K_{A}$ and $I K_{s}$ were acquired by the protocol A. Sustained current $I K_{s}$ was activated by the protocol B. Currents sensitive to the conditioning voltage were obtained by subtraction of the currents derived with the protocol $\mathrm{B}$ from the currents with the protocol A. Voltage-dependent $\mathrm{Ca}^{2+}$ currents were elicited by voltage steps from a holding potential of $-60 \mathrm{mV}$ to $60 \mathrm{mV}$ in increments of $10 \mathrm{mV}$ of each step for $150 \mathrm{~ms}$. Linear leak and residual capacitive currents were subtracted off-line from all traces prior to analysis by adding scaled up leak currents generated from test potentials to $-100 \mathrm{mV}$ or digitally on-line subtracted by use of the $\mathrm{p} / 4$ protocol (pClamp6 user's guide, Axon Instruments, Union City, CA). The junction potential between the intracellular and extracellular solutions was corrected based on the method of Neher (Neher, 1992). Signals were filtered at $5 \mathrm{kHz}$, digitized at $2-10 \mathrm{kHz}$ by Digidata 1200 or 1320 interface, acquired by pClamp6, and analyzed off-line by pClamp8 (Axon Instruments, CA).

\subsubsection{Synaptic stimulation}


Synaptic projections to the recorded neurons were activated by electrical stimulation using a bipolar-tungsten electrode placed on the ipsilateral lateral-dorsal part of the ICC or on the CoIC (see Results below). Synaptic responses were evoked by a $0.1 \mathrm{~ms}$ square wave pulse produced by a stimulator (S-8800; Grass, West Warwick, RI, USA) and delivered through an isolation unit. The cell membrane potential was clamped at $-60 \mathrm{mV}$ during the recordings of synaptic responses.

\subsubsection{Solutions and drugs}

Unless indicated otherwise, a standard recording ACSF was used. It contained (in mM): $129 \mathrm{NaCl}, 3 \mathrm{KCl}, 1.2 \mathrm{KH}_{2} \mathrm{PO}_{4}, 2.4 \mathrm{CaCl}_{2}, 1.3 \mathrm{MgSO}_{4}, 20 \mathrm{NaHCO}_{3}, 3 \mathrm{HEPES}$, and 10 glucose, with a pH of 7.4 and an osmolarity of $290-310 \mathrm{mOsm} / \mathrm{kg}$. Outward $\mathrm{K}^{+}$ currents were recorded in ACSF containing TTX (selective voltage-dependent $\mathrm{Na}^{+}$ channel blocker, $1 \mu \mathrm{M})$ and low $\mathrm{Ca}^{2+}(0.1 \mathrm{mM})$. To evoke voltage dependent $\mathrm{Ca}^{2+}$ currents, the perfusion medium was switched to a solution containing (mM): 20 TEA, 5 4-AP, $129 \mathrm{NaCl}, 3 \mathrm{KCl}, 2.4 \mathrm{CaCl}_{2}, 1.3 \mathrm{MgSO}_{4}, 20 \mathrm{NaHCO}_{3}, 3$ HEPES and 10 glucose, plus $1 \mu \mathrm{M}$ TTX (pH 7.4 adjusted with $\mathrm{NaOH}$ ). AMPA receptor-mediated EPSCs were isolated by suppression of NMDA EPSCs by application of APV $(100 \mu \mathrm{M})$ in addition to bicuculline $(10 \mu \mathrm{M})$ which is the $\mathrm{GABA}_{\mathrm{A}}$ receptor antagonist and strychnine $(0.5 \mu \mathrm{M})$ which is the glycine receptor antagonist. NMDA receptor-mediated EPSCs were isolated by suppression of AMPA EPSCs by application of CNQX $(10 \mu \mathrm{M})$ in addition to bicuculline $(10 \mu \mathrm{M})$ and strychnine $(0.5 \mu \mathrm{M})$. GABA $A_{A}$ receptor-mediated IPSCs were separated by suppression of NMDA and AMPA EPSCs by application of APV $(100 \mu \mathrm{M})$ and CNQX $(10 \mu \mathrm{M})$. To separate postsynaptic $\mathrm{GABA}_{\mathrm{B}}$ receptor mediated responses, recordings were obtained in the presence of CNQX $(10 \mu \mathrm{M})$, APV $(100 \mu \mathrm{M})$, bicuculline 
$(10 \mu \mathrm{M})$, and strychnine $(0.5 \mu \mathrm{M})$. In some experiments, $\mathrm{BaCl}_{2}(1 \mathrm{mM})$ which is inward rectifier potassium channel blocker and 4-aminopyridine (4-AP, $2 \mathrm{mM}$ ) which is transient A-type potassium blocker were used. All drugs were given by bath application and they were obtained from Sigma Aldrich Company, except ZD7288 that was purchased from Tocris Cookson Ltd. (Ballwin, MO, USA) and CGP35348 that was generously provided by Novartis Inc. (Switzerland).

\subsubsection{Data analysis}

All measurements were made from the recordings at least 6-8 min after establishing a whole-cell configuration and showing a stable resting potential. Neurons with resting potentials more negative than $-55 \mathrm{mV}$ were accepted and used for further analysis. Input resistance was determined from the linear fit of neuronal voltage responses elicited by $200 \mathrm{~ms}$ hyperpolarizing current injections from 0 to $-80 \mathrm{pA}$. The current-voltage (IV) curve was plotted based on measurements between the resting membrane potential and the peak membrane potential in response to current injection. The amplitude of an action potential was obtained as the difference between the peak voltage of action potential and initial membrane potential at which the membrane potential started to rise rapidly. The spike half-width was measured at $1 / 2$ amplitude of an action potential (AP). The AP threshold was defined as the membrane potential at which the neuron generated only one or two spikes. The threshold current for firing was defined as the lowest level of positive current injection required to elicit at least one action potential. Firing frequency in $\mathrm{Hz}$ was determined by the total number of APs divided by the duration of the current injection. The amplitude of AHP was measured from the beginning of the spike to the lowest level of the AHP. The AHP half-width was 
quantified at $1 / 2$ amplitude of the AHP. The amplitude of the depolarizing sag during hyperpolarization induced by negative current injection was calculated as the difference between the peak and steady voltage defections. The amplitude of the rebound depolarization after membrane hyperpolarization was measured as the difference between the resting membrane potential and the peak depolarization. The theoretical equilibrium potential for $\mathrm{K}^{+}$was calculated using Nernst equation $\left(\mathrm{E}_{\mathrm{k}}=\mathrm{RT} / \mathrm{F}^{*} \ln [\mathrm{K}]_{\mathrm{o}} /[\mathrm{K}]_{\mathrm{i}}\right)$ with an activity coefficient of 0.75 for potassium gluconate (Vanysek, 1988; Liu and Leung, 2003).

To determine the effects of the drug, the mean amplitude of five postsynaptic currents (PSCs) taken immediately before application of the drug was compared with the mean of five consecutive PSCs taken during the drug and five PSCs taken after cessation of the drug. Measurements during or after drug application were taken 3-5 min following the change in the composition of the ACSF. The paired-pulse ratio (PPR) was calculated as the $\mathrm{P} 2 / \mathrm{P} 1$, where $\mathrm{P} 1$ and $\mathrm{P} 2$ were the amplitude of 1st and 2 nd evoked synaptic current, respectively.

Numerical averages are presented as means \pm SEMs. Statistical significance was evaluated by either two-tailed Student's $t$ tests or a repeat measure one-way ANOVA with a post hoc test, and the minimum criterion for statistical significance was set at $\mathrm{p}<0.05$. When multiple post hoc comparisons were performed, the alpha-level was Bonferroni adjusted.

\subsection{Results}

Recordings were obtained from 107 ICD neurons located at dorsal and medial part of the IC. The resting potential was stable throughout the recording. The mean 
resting membrane potential of these neurons was $-59.9 \pm 0.3 \mathrm{mV}$ (range, -56.0 to -64.5 $\mathrm{mV})$. Spontaneous action potentials were very rare in these cells.

\subsubsection{Effects of baclofen on passive membrane properties of ICD neurons}

Baclofen application reversibly induced a hyperpolarization and decreased the membrane input resistance in 40/57 (70.2\%) ICD neurons sampled. For each cell tested with baclofen, the maximum drug effect was usually observed within 3-5 min after drug application. The drug effect was typically washed out 4-6 min after returning to control ACSF. Figure 4.1A shows traces from a representative baclofen-sensitive neuron in response to six levels of current injection (from -100 to $20 \mathrm{pA}$ ) under current clamp mode. This cell had a resting membrane potential of $-61.5 \mathrm{mV}$ and an input resistance of 310.5 $\mathrm{M} \Omega$ in ACSF. During application of baclofen $(20 \mu \mathrm{M})$ the resting potential dropped to $68.0 \mathrm{mV}$ and membrane input resistance decreased to $220.0 \mathrm{M} \Omega$ (Middle traces). After washing with ACSF, the membrane potential and input resistance recovered to $-60.5 \mathrm{mV}$ and 325.0 M $\Omega$, respectively (Lower traces). Group data are shown in Figure 4.1B and C. Baclofen application $(20 \mu \mathrm{M})$ significantly hyperpolarized the cell membrane from $59.4 \pm 0.4 \mathrm{mV}$ to $-65.7 \pm 0.5 \mathrm{mV}(\mathrm{p}<0.001 ; \mathrm{n}=21$, Fig. $4.1 \mathrm{~B})$. The input resistance also decreased greatly with baclofen from $329.3 \pm 20.6 \mathrm{M} \Omega$ to $229.1 \pm 16.8 \mathrm{M} \Omega(\mathrm{p}<0.001 ; \mathrm{n}=26$, Fig. 4.1C).

Baclofen decreased the cell's resting potential and input resistance in a concentration-dependent manner. Figure $4.2 \mathrm{~A}_{1}$ shows changes in the resting potential of a representative ICD neuron before, during and after application of baclofen at 3, 20, $100 \mu \mathrm{M}$. Figure $4.2 \mathrm{~B}_{1}$ shows responses of this neuron to a hyperpolarizing current pulse 
of $80 \mathrm{pA}$ in ACSF and in baclofen at $3,20,100 \mu \mathrm{M}$. At $3 \mu \mathrm{M}$, the neuron hyperpolarized 6 $\mathrm{mV}$ and its input resistance decreased by $28 \%$ relative to the control. Application of 20 $\mu \mathrm{M}$ baclofen caused a hyperpolarization of $10.5 \mathrm{mV}$ and decreased the input resistance by $36 \%$ relative to the control. Baclofen at $100 \mu \mathrm{M}$ hyperpolarized the cell by $13.5 \mathrm{mV}$ and decreased the input resistance by $39 \%$ relative to the control. Normalized hyperpolarization, expressed as a percentage, was calculated as a ratio of the hyperpolarization at each concentration $(1,310,20$ and $50 \mu \mathrm{M})$ to that of the hyperpolarization at the concentration of $100 \mu \mathrm{M}$. With the same principle, I also normalized the reduction in the input resistance. As the concentration increased, the hyperpolarization and the reduction of the input resistance were progressively increased, indicating concentration-dependent effects of baclofen on ICD neurons (n=5; Fig. 4.2A and $2 \mathrm{~B}_{2}$ ). The concentration of baclofen that produced $50 \%$ of the maximum effect on the cell resting potential and input resistance was about $2-5 \mu \mathrm{M}$.

\subsubsection{Effects of baclofen on active membrane properties of ICD neurons}

\subsubsection{Effects of $G A B A_{B}$ receptor activation on firing behaviour of ICD neurons}

Next I will address whether activation of $\mathrm{GABA}_{\mathrm{B}}$ receptor modulates firing activity of ICD neurons. As all sampled ICD neurons did not exhibit spontaneous firing; thus, their firing activity was examined by injection of depolarizing current pulses at various levels. Most of the neurons displayed a sustained firing pattern in response to suprathreshold current pulses. Overall, the firing activity of $71.15 \%$ (37/52) ICD neurons tested was reversibly depressed by application of baclofen $(20 \mu \mathrm{M})$. A typical example of baclofen actions is shown in Figure 4.3A. In ACSF, the neuron showed a train of spikes 
in response to $120 \mathrm{pA}$ current pulse (top trace) with a resting membrane potential of $57.0 \mathrm{mV}$. Application of baclofen $(20 \mu \mathrm{M})$ markedly inhibited its firing discharge and hyperpolarized its resting membrane potential to $-62.5 \mathrm{mV}$ (middle trace). The effects of baclofen were reversible (bottom trace). To rule out the possibility that the suppression of the firing activity by baclofen might be due to membrane hyperpolarization, the resting membrane potential during baclofen application was corrected to near the original level by applying a DC current injection which brought the membrane potential to $-58 \mathrm{mV}$. Under this condition, application of baclofen $(20 \mu \mathrm{M})$ led to the similar inhibition on the firing activity as that when the cell's membrane potential was at $-62.5 \mathrm{mV}$ (Fig. 4.3B). These results suggest that the mechanisms other than membrane hyperpolarization may account for the suppression of the firing activity by baclofen. When the firing rate was plotted as a function of current level the firing rates at all levels of current injection were decreased by baclofen (Fig. 4.3C for the neuron shown in $A$ and $B$; Fig. 4.3D for all the neurons tested, $\mathrm{n}=37$ ). The suppression of the firing rate by baclofen was more obvious at higher levels of current injection.

4.3.2.2 Effects of $G A B A_{B}$ receptor activation on action potential properties of ICD neurons

The analysis of effects of $\mathrm{GABA}_{B}$ receptor activation on action potentials was performed on 37 ICD neurons that were subjected to baclofen. For these experiments, neurons were current-clamped at resting membrane potential throughout the whole recording period. A typical example is illustrated in Figure 4.4A. In ACSF, when a $60 \mathrm{pA}$ current pulse was delivered, the membrane was depolarized with a hump at the beginning of the depolarization. As the level of depolarizing current was increased to $80 \mathrm{pA}$, the 
depolarization became larger and passed the threshold for an AP, i.e., $-46.5 \mathrm{mV}$. As a result, an AP was generated at the early phase of depolarization (Fig. 4.4A $\mathrm{A}_{1}$ ). I defined 80 $\mathrm{pA}$ as the threshold current for an AP for this neuron. After application of baclofen $(20 \mu \mathrm{M})$ the membrane depolarization was reduced and no hump was induced in response to $60 \mathrm{pA}$ and no AP generated in response to $80 \mathrm{pA}$ current pulse (Fig. 4.4A $\mathrm{A}_{2}$ ). In fact, the threshold current was increased to $120 \mathrm{pA}$ and the threshold membrane potential for an AP became more positive, i.e., $-41.5 \mathrm{mV}$. Furthermore, baclofen also affected the AP itself (Fig. 4.4A $\mathrm{A}_{3}$ ). After application of baclofen, the AP amplitude was reduced from $58.5 \mathrm{mV}$ to $56.2 \mathrm{mV}$ and the AHP amplitude was decreased from $15 \mathrm{mV}$ to $9 \mathrm{mV}$, while the AP half-width was increased from $1.2 \mathrm{~ms}$ to $1.4 \mathrm{~ms}$. The similar effects of baclofen on the threshold current, the threshold membrane potential, AP amplitude and half-width, and AHP amplitude and half-width were observed in 28 neurons tested. The mean AP threshold during baclofen application was more positive $(-31.6 \pm 1.6 \mathrm{mV})$ than that in ACSF $(-41.5 \pm 1.7 \mathrm{mV}(\mathrm{p}<0.001)$, and the threshold current during baclofen $(125.9 \pm 13.9$ pA) was much larger than that in ACSF $(60.0 \pm 10.6 \mathrm{pA})(\mathrm{p}<0.001$; Fig. 4.4B). The AP amplitude was significantly reduced by baclofen application (before: $47.8 \pm 2.6 \mathrm{mV}$; during: $44.6 \pm 2.5 \mathrm{mV}$; $\mathrm{p}<0.05$; Fig. $4.4 \mathrm{C}$, left panel). The AP half-width during baclofen application $(1.4 \pm 0.1 \mathrm{~ms})$ was markedly increased compared to that before baclofen application $(1.3 \pm 0.1 \mathrm{~ms})(\mathrm{p}<0.05$; Fig. $4.4 \mathrm{C}$, right panel). The mean AHP amplitude and AHP half-width before baclofen application were $12.9 \pm 0.9 \mathrm{mV}$ and $69.0 \pm 5.7 \mathrm{~ms}$, respectively. After baclofen application the mean AHP amplitude and AHP half-width were decreased to $8.5 \pm 0.6 \mathrm{mV}$ and $39.9 \pm 6.5 \mathrm{~ms}$, respectively ( $\mathrm{p}<0.01$; Fig. 4.4D). In addition, it is worthwhile mentioning that the slow AHP (arrow head) was greatly 
compressed, and a short depolarization (arrow) appeared immediately during the following phase of the AP after application of baclofen in 5/52 ICD cells tested (Fig. 4.4E).

\subsubsection{Baclofen evoked an inward rectifying $\mathrm{K}^{+}$current}

To identify the current induced by baclofen, both electrophysiological and pharmacological experiments were performed in 16 ICD neurons. Figure 4.5A and $\mathrm{B}$ illustrates responses of an ICD neuron to voltage steps and the corresponding $I-V$ plots, respectively. In ACSF, the neuron expressed an inward current evoked by a series of voltage steps from $-60 \mathrm{mV}$ to $-150 \mathrm{mV}$ (500 ms duration) (Fig. 4.5A upper traces, arrow head). Following application of $20 \mu \mathrm{M}$ baclofen, the baseline current was shifted positively by $65 \mathrm{pA}$, and the magnitude of the evoked inward currents increased, especially at higher voltage steps (Fig. 4.5A middle traces, filled circle). The changes of the evoked inward currents almost dissipated with washout of baclofen (Fig. 4.5A lower traces, open circle). The I-V relationships before, during and after baclofen application were then plotted (Fig. 4.5B). The baclofen-induced current was derived by substraction of the current in ACSF from that during baclofen application. Figure 4.5C illustrates the baclofen-induced currents in response to the voltage steps from -60 to $-150 \mathrm{mV}(-10$ $\mathrm{mV} / \mathrm{step}$ ) in $16 \mathrm{ICD}$ neurons. The baclofen-induced current was reversed at $-81.36 \mathrm{mV}$, which is close to the $\mathrm{K}^{+}$equilibrium potential $\left(\mathrm{E}_{\mathrm{k}+}\right)$ of $-79.21 \mathrm{mV}$ calculated with $\mathrm{K}^{+}$ concentrations used in the present study.

Based on the evidence that the heavy metal barium acts to block the inward rectifier $\mathrm{K}^{+}$channel currents (Sodickson and Bean, 1998), the effects of baclofen were 
tested by $1 \mathrm{mM} \mathrm{BaCl}_{2}$ in 5 ICD neurons. A representative example is shown in Figure 4.6. Figure 4.6A displays responses to a series of voltage steps from -60 to $-140 \mathrm{mV}(-10$ $\mathrm{mV} / \mathrm{step}$, duration $300 \mathrm{~ms}$ ) in ACSF. The slice was then treated with $20 \mu \mathrm{M}$ baclofen for $5 \mathrm{~min}$, which resulted in a significant increase in the membrane conductance and a baseline current shift of $35 \mathrm{pA}$ (Fig. 4.6B). An addition of barium suppressed the baclofen-induced changes substantially (Fig. 4.6C). The I-V curves were plotted under conditions of ACSF, baclofen, baclofen plus barium and wash (Fig. 4.6E). These plots indicate that barium blocked the effect of baclofen reversibly. The baclofen-induced current was almost abolished by the application of barium (Fig. 4.6F).

In sum, the above results of the blockade of baclofen-induced current by barium and the reversal potential of the baclofen-induced current strongly suggest that baclofen activates a postsynaptic inward rectifying $\mathrm{K}^{+}$current in ICD neurons.

\subsubsection{CGP35348 significantly reduced the baclofen mediated inhibition}

To verify the involvement of $\mathrm{GABA}_{\mathrm{B}}$ receptors in baclofen-induced inhibition, the selective $\mathrm{GABA}_{\mathrm{B}}$ receptor anatagonist CGP35348 was applied to 8 ICD neurons. CGP35348 reversibly blocked the baclofen-induced hyperpolarization and decrease in the membrane input resistance. Figure 4.7A displays an example recorded in current clamp mode. In ACSF, negative current pulses from -20 to $-300 \mathrm{pA}$ caused hyperpolarization of the cell membrane. The input resistance of this cell was $325 \mathrm{M} \Omega$. When a hyperpolarization was large enough, a rebound depolarization with associated spikes were generated after hyperpolarization. Positive current steps from 20 to $100 \mathrm{pA}$ depolarized the cell membrane. One or several spikes were generated when the 
membrane potential reached or passed the threshold of the action potential (upper traces). Application of baclofen $(20 \mu \mathrm{M})$ hyperpolarized the cell by $5 \mathrm{mV}$. The rebound depolarization and associated spikes were abolished. The firing during depolarizing current injection was also depressed. The input resistance was decreased from 325 to 225 $\mathrm{M} \Omega$ (middle traces). Co-application of CGP35348 $(100 \mu \mathrm{M})$ with baclofen reversed the effects of baclofen. The resting membrane potential was back to $-59 \mathrm{mV}$ and the input resistance was also recovered to $350 \mathrm{M} \Omega$. The rebound and spikes appeared again.

The antagonism of baclofen-induced currents by CGP35348 was also observed in 5 ICD neurons under voltage clamp conditions. A typical example was illustrated in Figure 4.7B and $\mathrm{C}$. In ACSF, hyperpolarizing voltage steps of $-10 \mathrm{mV}$ increment from a holding potential of $-60 \mathrm{mV}$ elicited instantaneous inward currents and slow inward currents (more details in next section). Application of baclofen $(20 \mu \mathrm{M})$ increased the membrane conductance and the inward currents. When CGP3548 $(100 \mu \mathrm{M})$ was coadministrated with baclofen, the membrane conductance was almost the same as that in ACSF. The I-V relationships shown in Figure 4.7C (upper panel) indicate that CGP35348 antagonized the effects of baclofen. Baclofen-evoked currents were greatly reduced by CGP35348 (Fig. 4.7C, lower panel). The above results confirm that the effects of baclofen were produced by activation of postsynaptic $\mathrm{GABA}_{\mathrm{B}}$ receptors.

\subsubsection{Modulation of $\mathrm{I}_{h}$ by $\mathrm{GABA}_{\mathrm{B}}$ receptor activation}

Under current clamp mode, many ICD neurons tested with injection of hyperpolarizing current displayed a slowly developing sag of the membrane potential, i.e., inward rectification. Figure 4.8A shows a typical example. Hyperpolarizing current 
pulses of the sufficient magnitude evoked a depolarizing sag of the membrane potential toward the resting value (upper traces). The rate and magnitude of the sag increased with the amplitude of the hyperpolarizing current injected. This sag was blocked almost completely by $100 \mathrm{nM} Z \mathrm{ZD} 7288(\mathrm{n}=5$; lower traces). With voltage clamp in the same cell as shown in Figure 4.8A, hyperpolarizing voltage steps $(-10 \mathrm{mV} / \mathrm{step})$ from a holding potential of $-60 \mathrm{mV}$ elicited an instantaneous inward current (Fig. 4.8B, upper taces), and a slowly activating inward current when the membrane potential was more negative than $-70 \mathrm{mV}$. This slowly activating inward current is thought to underlie the sag observed in current clamp mode. The amplitude of the peak current (measured immediately after the capacitive transient; $\mathbf{\Delta}$ ) and the steady current (measured at the end of the voltage step; $\bullet$ ) were plotted as a function of voltage steps (Fig. 4.8C, upper panel). The difference between the peak and steady current represented the current that was activated by the hyperpolarizing step (Fig. 4.8C, lower panel). Application of $100 \mathrm{nM}$ ZD7288 blocked almost completely the current activated by the hyperpolarization $(n=5)$ (Fig. 4.8B, lower traces and $\mathrm{C}$ ). These results suggest that hyperpolarization in some ICD neurons can induce $\mathrm{I}_{h}$.

Next, I studied the effects of activation of $\mathrm{GABA}_{\mathrm{B}}$ receptor on $\mathrm{I}_{h}$. Figure $4.8 \mathrm{D}$ shows an example recorded under current clamp mode. In ACSF, a hyperpolarizing current of $-200 \mathrm{pA}$ led to an initial hyperpolarization followed by a slowly depolarizing sag (left panel). Bath application of baclofen $(20 \mu \mathrm{M})$ reversibly reduced the hyperpolarization and blocked most of the depolarizing sag (right panel). To rule out the possibility that the depression of the amplitude of depolarizing sag by baclofen was due to a smaller hyperpolarization, the amount of current injection was adjusted to a larger 
negative value during baclofen application in order to generate the same peak level of hyperpolarization as that when the slice was in ACSF (Fig. 4.8E). No obvious sag was found with the higher hyperpolarizing current injection. This result indicates that baclofen can directly modulate $\mathrm{I}_{h}$ in ICD neurons. Furthermore, the action of baclofen on the cell membrane was not only through the modulation of $\mathrm{I}_{h}$, but also inward currents in the neurons that had $\mathrm{I}_{h}$. An example is shown in Figure 4.8F. With a holding potential of $-60 \mathrm{mV}$, an obvious activation of $\mathrm{I}_{h}$ was induced by a hyperpolarizing step of $-90 \mathrm{mV}$ (300 ms duration) in ACSF. Application of $100 \mathrm{nM}$ ZD7288 blocked the $\mathrm{I}_{h}$. An addition of $20 \mu \mathrm{M}$ baclofen caused an increase in the inward current even in the absence of $\mathrm{I}_{h}$. These results indicate that the inhibition of $I_{h}$ by baclofen is independent from activation of inward rectifier potassium channels.

\subsubsection{Modulation of rebound depolarization by $\mathrm{GABA}_{\mathrm{B}}$ receptor activation}

The rebound depolarization following membrane hyperpolarization is one of the important intrinsic membrane properties of ICD neurons. More than half of the ICD neurons display the rebound depolarization and associated APs upon the release of membrane hyperpolarization, which has been described in detail in Chapter 2 and 3. The effects of $\mathrm{GABA}_{B}$ receptor activation on the rebound depolarization are demonstrated below. Bath application of baclofen reversibly suppressed the rebound depolarization (Fig. 4.9A). Note that the input resistance of this neuron was also reduced by application of baclofen. As we have known that the amplitude of the rebound depolarization depends on the amplitude and duration of the hyperpolarization. To rule out the possibility that the depression of the amplitude of the rebound depolarization was due to the smaller 
hyperpolarization during baclofen application, I adjusted the current amount to a larger negative value in order to generate the same level of hyperpolarization in baclofen solution as that in ACSF (Fig. 4.9B). Baclofen reduced the amplitude of the rebound depolarization in a similar degree in both conditions. These results suggest that the suppression of the rebound by activation of $\mathrm{GABA}_{\mathrm{B}}$ receptor is not due to the decrease in the input resistance. On average, the amplitude of the rebound depolarization (the amplitude of the rebound was measured at the current level just before an anode break spike could be generated.) was decreased by $73.6 \%$ following $20 \mu \mathrm{M}$ baclofen application (control: $9.5 \pm 1.0 \mathrm{mV}$; baclofen: $2.5 \pm 0.8 \mathrm{mV}$; wash: $8.8 \pm 1.1 \mathrm{mV}$; $\mathrm{n}=12, \mathrm{p}<0.001$; Fig. 4.9C). In voltage clamp mode, baclofen caused a suppression of low-threshold calcium current induced by prehyperpolarization. Figure 4.9D shows a typical example. The cell was hold at $-60 \mathrm{mV}$. Then a $-110 \mathrm{mV}$ hyperpolarized pulse $(300 \mathrm{~ms})$ was applied prior to returning back to $-60 \mathrm{mV}$. An inward current (arrow, black trace) was induced upon the release of the voltage pulse in ACSF (Fig. 4.9D $\mathrm{D}_{1}$ ). This inward current is thought to be mediated by low threshold T type calcium channels (Slugg et al., 2003). After application of baclofen the inward current was depressed (grey trace). Figure $4.9 \mathrm{D}_{2}$ displays the relationship between the hyperpolarization level and the amplitude of the inward current. As the hyperpolarization was increased from -70 to $-120 \mathrm{mV}$, a larger inward current was induced (filled circle). Bath application of baclofen depressed the inward current at all the levels of hyperpolarization with more obvious effects at higher levels of hyperpolarization (open circle).

\subsubsection{Modulation of depolarization hump by activation of $\mathrm{GABA}_{\mathrm{B}}$ receptors}


The depolarization hump is another distinct intrinsic membrane property of ICD neurons. The hump is important particularly around the firing threshold because it can promote synaptic inputs to generate spikes. Therefore, the effects of activation of $\mathrm{GABA}_{B}$ receptor on the subthreshold hump were investigated. In Figure $4.10 \mathrm{~A}_{1}$ (top trace), a positive current pulse (180 $\mathrm{pA}, 200 \mathrm{~ms}$ duration) elicited a depolarization with a hump appeared at the beginning of the depolarization (arrow). When baclofen $(20 \mu \mathrm{M})$ was added to the ACSF, the hump was reduced (middle trace, arrow), but recovered after wash (bottom trace, arrow). To rule out the possibility that the depression of the hump was due to the smaller depolarization in baclofen, the amount of current was adjusted to a larger value in order to generate the same level of depolarization as that when the slice was in ACSF (Fig. 4.10A $\mathrm{A}_{2}$ ). However, the amplitude of the hump was still obviously decreased in baclofen compared to that in ACSF. Application of baclofen significantly reduced the amplitude of the hump at all subthreshold levels of current injection (Fig. $\left.4.10 \mathrm{~A}_{3}\right)$. It has been demonstrated that the hump is mediated by voltage-dependent calcium channels (Li et al., 1998). To study further how $\mathrm{Ca}^{2+}$ current is affected by activation of $\mathrm{GABA}_{\mathrm{B}}$ receptors, $\mathrm{Ca}^{2+}$ currents were isolated and examined before and during application of baclofen. Figure 4.10B displays inward currents induced by a voltage step from a holding potential of $-60 \mathrm{mV}$ to a voltage step of $-10 \mathrm{mV}$ in the presence of the $\mathrm{Na}^{+}$channels blocker, $1 \mu \mathrm{M}$ TTX, and the $\mathrm{K}^{+}$channel blockers, $20 \mathrm{mM}$ TEA and 4-AP $(2 \mathrm{mM})$. When baclofen $(20 \mu \mathrm{M})$ was added to the control solution, the inward current was depressed (Fig. $4 \cdot 10 \mathrm{~B}_{1}$ ). The peak inward currents were plotted as a function of voltage steps in the control (filled circle) and baclofen solution (filled triangle) (Fig. $4.10 \mathrm{~B}_{2}$ ). In control solution, an inward current was activated at $-50 \mathrm{mV}$ and peaked 
at $-10 \mathrm{mV}$. Application of baclofen reduced the $\mathrm{Ca}^{2+}$ current without change of the level at which the peak current was reached.

\subsubsection{Inhibition of $A$-current by activation of $\mathrm{GABA}_{B}$ receptors}

In ICD, many neurons show a buildup firing pattern that has been known to attributable to activation of transient potassium channels (A-current) (Sivaramakrishnan and Oliver, 2001). The effects of activation of $\mathrm{GABA}_{\mathrm{B}}$ receptor on the buildup firing pattern are shown in Figure 4.11. Figure 4.11A illustrates an ICD neuron having a buildup firing pattern in response to depolarizing current pulse. The buildup firing pattern was characterized by a delay (arrow) of firing in response to an injection of depolarizing current of $150 \mathrm{pA}$ (upper trace). Another neuron shown in Figure 4.11B (upper trace) displays similar firing responses to an injection of depolarizing current (180 pA) following a hyperpolarizing current of $-200 \mathrm{pA}$. Application of 4-AP ( $2 \mathrm{mM})$, a selective blocker of A-current, eliminated the delay of firing in both neurons (Fig. 4.11A and B, lower traces), suggesting that activation of A-current accounts for the delay of firing. Figure 4.11C and D show that application of baclofen $(20 \mu \mathrm{M})$ markedly prolonged the delay of firing in both neurons (from $57.4 \mathrm{~ms}$ to $156.5 \mathrm{~ms}$ for neuron $\mathbf{A}$ and $158.1 \mathrm{~ms}$ to 254.1 ms for neuron B). Similar results were obtained from 5 buildup neurons that had a delay of firing in response to depolarizing current only and 11 buildup neurons that had a delay of firing in response to depolarizing current following hyperpolarizing current.

To examine further the direct effects of activation of $\mathrm{GABA}_{\mathrm{B}}$ receptor on $\mathrm{A}$ currents, voltage clamp recordings were performed in 5 neurons showing a buildup firing pattern. Neurons were recorded in the presence of TTX $(1 \mu \mathrm{M})$ and low $\mathrm{Ca}^{2+}$ 
concentration $(0.1 \mathrm{mM})$. Separation of different types of $\mathrm{K}^{+}$current was achieved by running voltage steps with different conditioning prepulse. Two distinct voltagedependent $\mathrm{K}^{+}$currents were identified, including A-current $\left(I K_{A}\right)$ and sustained $\mathrm{K}^{+}$ current $\left(I K_{S}\right)$ in ICD neurons. To activate the $I K_{A}$, the neuron was first hold at $-60 \mathrm{mV}$ for $10 \mathrm{~ms}$ and then stepped to $-100 \mathrm{mV}$ for $500 \mathrm{~ms}$ (conditioning prepulse potential), which was followed by a depolarizing voltage step of $+30 \mathrm{mV}$ for $300 \mathrm{~ms}$. As a result, an outward current with a transient, inactivating component followed by a non-inactivating component was activated (Fig. $4.12 \mathrm{~A}_{1}$, lower trace). The total voltage-dependent $\mathrm{K}^{+}$ current $\left(I K_{T}\right)$ included $I K_{A}$ and $I K_{S}$ components. To isolate $I K_{A}$, the conditioning prepulse potential was then set to $-40 \mathrm{mV}$. This protocol did not remove inactivation of transient $\mathrm{K}^{+}$channels and thus $I K_{A}$ was not activated during the voltage step to $+30 \mathrm{mV}$ (Fig. $4.12 \mathrm{~A}_{2}$, lower trace). Therefore, the outward current represented $I K_{S}$ only. The $I K_{A}$ was then derived by subtraction of $I K_{S}$ from $I K_{T}$ (Fig. 4.12A, right panel, lower trace). Application of baclofen $(20 \mu \mathrm{M})$ significantly potentiated all of the voltage-dependent $\mathrm{K}^{+}$ currents including the $I K_{A}$ (from $775.0 \mathrm{pA}$ to $1287.2 \mathrm{pA}$ ), $I K_{S}$ (from $651.6 \mathrm{pA}$ to $903.6 \mathrm{pA}$ ) and $I K_{T}$ (from $1426.6 \mathrm{pA}$ to $2190.8 \mathrm{pA}$ ) (Fig. 4.12A, 3 panels, upper traces). Similar potentiations were found in 5 neurons tested. In Figure $4.12 \mathrm{~B}$ and $\mathrm{C}$, the amplitude of $I K_{A}$ and $I K_{S}$ of this neuron was plotted as a function of voltage steps, respectively. The peak amplitude of both $I K_{A}$ and $I K_{S}$ was increased with increasing levels of depolarization, and the current-voltage relationship was sigmoidal. Baclofen administration enhanced both $I K_{A}$ and $I K_{S}$ with more obvious increase in $I K_{A}$ amplitude, especially at higher voltage steps. 


\subsubsection{Stimulus-evoked postsynaptic $\mathrm{GABA}_{\mathrm{B}}$ receptor-mediated response}

So far, the data have demonstrated that functional $\mathrm{GABA}_{\mathrm{B}}$ receptors are present on postsynaptic sites of ICD neurons, but it is still unclear whether they can be activated by GABA during normal physiological processes, i.e., whether the amount of GABA released is enough to reach the $\mathrm{GABA}_{B}$ receptors. To address this question, electrical stimulation of presynaptic terminals in the CoIC was applied and postsynaptic responses were examined. Single pulse $(0.1 \mathrm{~ms}$ duration) and repetitive stimulation (10 pulses of $0.1 \mathrm{~ms}$ each, frequency $200 \mathrm{~Hz}$ ) were delivered and recordings were made in voltage clamp mode in the presence of the ionotropic glutamate receptor antagonists D-APV $(100 \mu \mathrm{M})$ and CNQX $(10 \mu \mathrm{M})$, and $\mathrm{GABA}_{\mathrm{A}}$ receptor antagonist bicuculline $(10 \mu \mathrm{M})$. The locations of the stimulation and recording were illustrated in Figure 4.13A. No IPSCs $(n=8)$ were observed following single stimulation of the CoIC (Fig. 4.13B, upperleft trace). In contrast, repetitive stimulation of the CoIC elicited pronounced slow IPSCs in 5/8 ICD neurons tested. The IPSCs had a mean duration of $0.8 \pm 0.2 \mathrm{~s}$, and a peak amplitude of $45.7 \pm 9.1 \mathrm{mV}$ at $0.16 \pm 0.03 \mathrm{~s}$ from the onset of the response (n=5). A typical example of the slow IPSC is shown in Figure 4.13B (right-upper trace). The amplitude of the slow IPSCs was greatly reduced to $5.6 \pm 3.1 \mathrm{mV}(12.3 \%$ of the control, $\mathrm{n}=5)$ after application of the $\mathrm{GABA}_{\mathrm{B}}$ receptor antagonist, CGP35348 (100 $\left.\mu \mathrm{M}\right)$ (Fig. 4.13C, lowerleft trace). The slow IPSC was reversed at about $-90 \mathrm{mV}$ which was close to the expected equilibrium potential for $\mathrm{K}^{+}$( $\mathrm{n}=3$; Fig. 4.13D, E). These results suggest that postsynaptic $\mathrm{GABA}_{\mathrm{B}}$ receptors can mediate slow synaptic inhibition in ICD neurons. 


\subsubsection{Presynaptic GABA $_{B}$ receptors modulated neuronal transmission in ICD} neurons

4.3.10.1 Synaptic responses mediated by $A M P A, N M D A$ and $G A B A_{A}$ receptors in $I C D$ neurons

To investigate the nature of synaptic responses in the ICD, 29 neurons were recorded under voltage clamp mode with electrical stimulation of single pulse to the inputs from the ipsilateral ICC as shown in Figure 4.14A. First, I classified the synaptic responses as either excitatory, inhibitory or mixed responses without any pharmacological manipulation. As shown in Figure 4.14B, 41.38\% (12/29) of the cells were predominantly excitatory, while $27.59 \%(8 / 29)$ of the cells displayed predominantly inhibitory responses. Mixed response was found in $31.03 \%(9 / 29)$ of the ICD neurons. Next, selective pharmacological receptor antagonists were applied to identify the types of synaptic receptors that mediated different kinds of synaptic responses. Figure $4.14 \mathrm{C}$ shows synaptic responses of a representative ICD neuron. In ACSF, single electrical stimulation of synaptic inputs from the ICC elicited an excitatory postsynaptic current (EPSC). This response shows a fast rising time and a relative slow decay time. After application of $10 \mu \mathrm{M}$ bicuculline, the selective $\mathrm{GABA}_{\mathrm{A}}$ receptor antagonist, the EPSC became larger and longer, indicating the contribution of the $\mathrm{GABA}_{\mathrm{A}}$ receptors to the response. A previous study using histological techniques has demonstrated that expression of glycine receptors was not found in ICD neurons (Merchan et al., 2005). Thus, remaining response was likely only excitatory and therefore further tested with glutamatergic receptor antagonists. After addition of $100 \mu \mathrm{M}$ APV, the specific NMDA receptor antagonist, the late part of the EPSC was suppressed, while the early part was 
still present. The remaining EPSC was completely blocked by adding $10 \mu \mathrm{M}$ CNQX, the AMPA receptor antagonist. These results suggest that a brief stimulation of the inputs from the ICC elicited inhibitory responses that are mediated by $\mathrm{GABA}_{\mathrm{A}}$ receptors, and excitatory responses that are mediated by both AMPA and NMDA receptors in ICD neurons.

4.3.10.2 Activation of presynaptic $G A B A_{B}$ receptors inhibited synaptic transmission in the ICD

Next, I investigated the role of presynaptic $\mathrm{GABA}_{\mathrm{B}}$ receptors in GABAergic and glutamatergic synaptic transmissions by application of baclofen. To examine the effects of presynaptic $\mathrm{GABA}_{\mathrm{B}}$ receptors by baclofen, the modified internal solution containing TEA, CsF and QX314 in the recording electrodes was used to block the $\mathrm{K}^{+}$channels that might be activated by baclofen acting on postsynaptic $\mathrm{GABA}_{\mathrm{B}}$ receptors. First, I investigated the modulation of AMPA and NMDA receptor-mediated EPSCs by activation of presynaptic $\mathrm{GABA}_{\mathrm{B}}$ receptors. Figure 4.14D and $\mathrm{E}$ illustrate the results from two typical examples. AMPA EPSCs were isolated by suppression of activation of $\mathrm{GABA}_{\mathrm{A}}$, glycine and NMDA receptors (Fig. 4.14D, upper trace). NMDA EPSCs were isolated by suppression of activation of $\mathrm{GABA}_{\mathrm{A}}$, glycine and AMPA receptors (Fig. 4.14E, upper trace). Bath application of $5 \mu \mathrm{M}$ baclofen significantly reduced both the AMPA and NMDA mediated EPSCs. Baclofen reduced the amplitude of AMPA EPSC from $151.67 \mathrm{pA}$ to $20.17 \mathrm{pA}$ (Fig. 4.14D, middle trace). The amplitude of NMDA EPSC was decreased from $233.76 \mathrm{pA}$ to $17.09 \mathrm{pA}$ (Fig. 4.14E, middle trace). The mean reduction for AMPA EPSCs was $61.33 \pm 6.91 \%(n=5)$, and that for NMDA EPSCs was $78.83 \pm 3.49 \%(n=8)$. To verify the involvement of postsynaptic $\mathrm{GABA}_{B}$ receptors in the 
modulation of EPSCs, CGP35348, the selective $\mathrm{GABA}_{\mathrm{B}}$ receptor antagonist, was applied in 8 ICD neurons. As shown in Figure 4.14D and E, the suppression of the EPSCs by baclofen was blocked by co-application of $100 \mu \mathrm{M}$ CGP35348. Similar effects of CGP35348 were found in other cells (for AMPA EPSCs, $n=3$; for NMDA EPSCs, $n=5$ ). These results suggest that baclofen suppresses glutamatergic transmission in ICD neurons and the effects were produced by activation of presynaptic $\mathrm{GABA}_{B}$ receptors.

Modulation of GABAergic IPSCs by presynaptic $\mathrm{GABA}_{\mathrm{B}}$ receptors was studied in 5 ICD neurons. A representative example is illustrated in Figure 4.15. A GABA IPSC $_{A}$ was isolated pharmacologically by adding $10 \mu \mathrm{M}$ CNQX and $100 \mu \mathrm{M}$ APV to the ACSF (Fig. 4.15A, upper trace). Application of baclofen $(5 \mu \mathrm{M})$ to the control solution reduced the IPSC amplitude from $104.7 \mathrm{pA}$ to $44.9 \mathrm{pA}$ (Fig. 4.15A, middle trace). The reduction in IPSC amplitude was almost blocked by $100 \mu \mathrm{M}$ CGP35348 (Fig. 4.15A, bottom trace). To demonstrate further that inhibition of $\mathrm{GABA}_{\mathrm{A}}$ IPSCs by activation of $\mathrm{GABA}_{\mathrm{B}}$ receptors was due to a presynaptic mechanism, I applied paired-pulse stimulations (200 ms interval). Figure 4.15B shows a typical example. In control solution containing CNQX and APV, the second IPSC was smaller than the first one (upper trace). The PPR was 0.63 . After application of $5 \mu \mathrm{M}$ baclofen, the reduction in the first IPSC (from 108.3 $\mathrm{pA}$ to $57.8 \mathrm{pA}$ ) was more obvious than that of the second IPSC (from $68.3 \mathrm{pA}$ to 56.4 $\mathrm{pA})$. The PPR was increased to 0.97 . The results indicate that activation of presynaptic $\mathrm{GABA}_{\mathrm{B}}$ receptors can modulate $\mathrm{GABA}_{\mathrm{A}}$ receptor mediated inhibition in the ICD. 


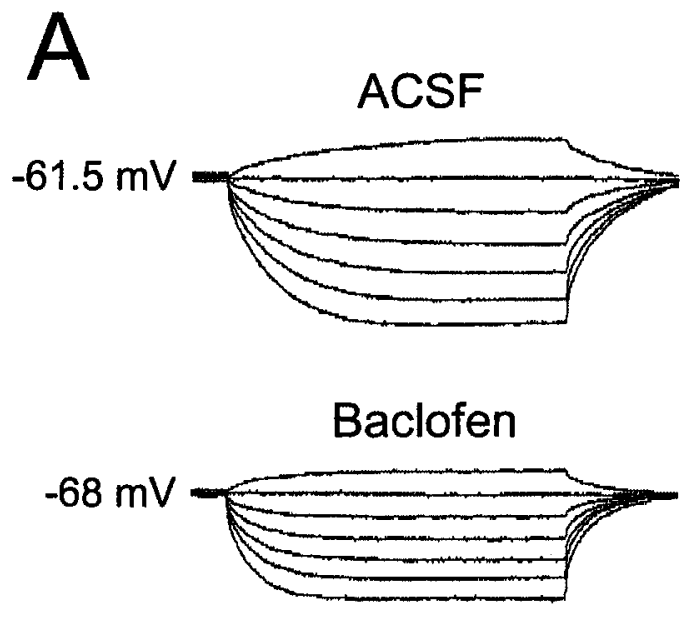

B
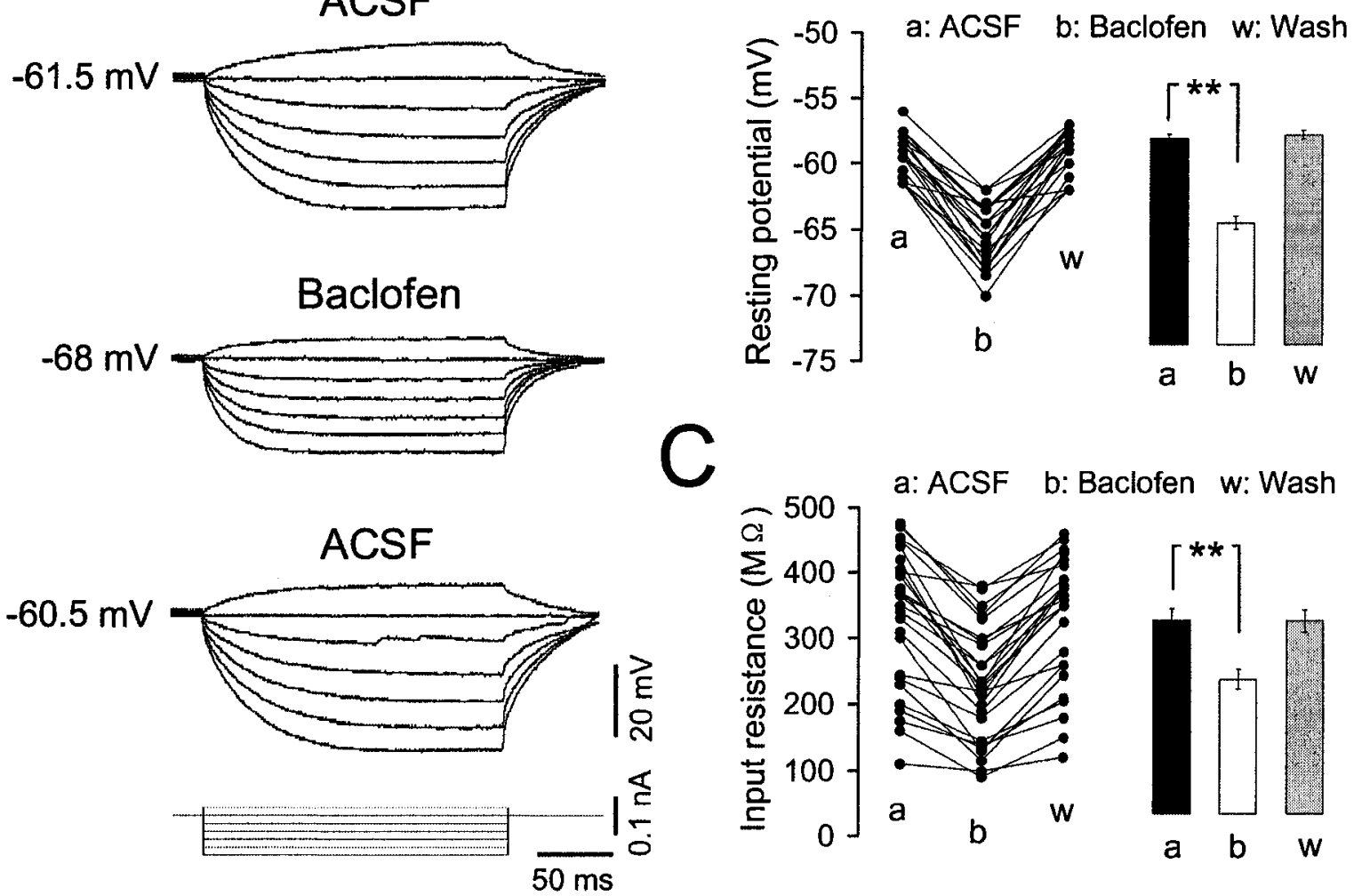

Figure 4.1. 
Figure 4.1. Baclofen hyperpolarized the cell membrane and decreased the input resistance in ICD neurons. A: Examples of responses to a series of current steps (from $100 \mathrm{pA}$ to $20 \mathrm{pA}, 20 \mathrm{pA} / \mathrm{step}$ ) before (upper traces), during (middle traces), and after (bottom traces) application of $20 \mu \mathrm{M}$ baclofen in an ICD neuron under whole-cell current clamp mode. The resting membrane potential was labelled beside the traces. B: Group data illustrating the change in the resting membrane potential when the cell was recorded in $\operatorname{ACSF}(a), 20 \mu \mathrm{M}$ baclofen $(b)$, and wash $(w)$. Left panel shows data for individual cells and right panel displays the average data for each group $(* * P<0.001, \mathrm{n}=21)$. C: Group data showing the change in the input resistance when the cell was recorded in $\operatorname{ACSF}(a), 20 \mu \mathrm{M}$ baclofen $(b)$, and wash (w). Left panel illustrates data for individual cells and right panel displays the average data for each group $(* * P<0.001, \mathrm{n}=26)$. 


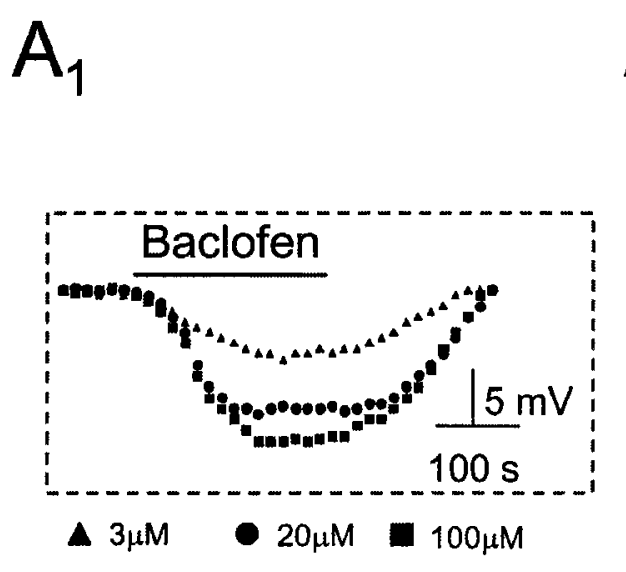

$\mathrm{A}_{2}$

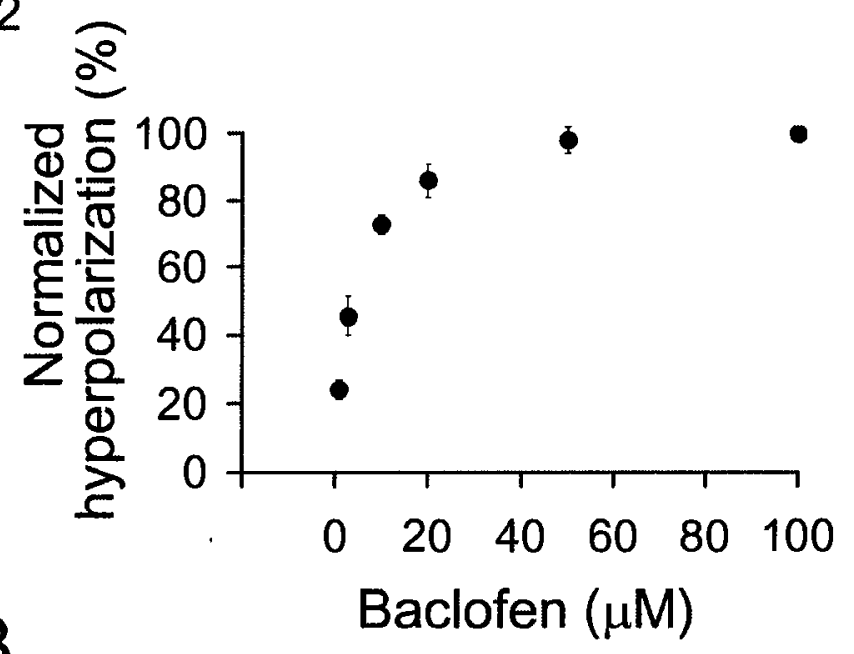

$\mathrm{B}_{1}$

$\mathrm{B}_{2}$

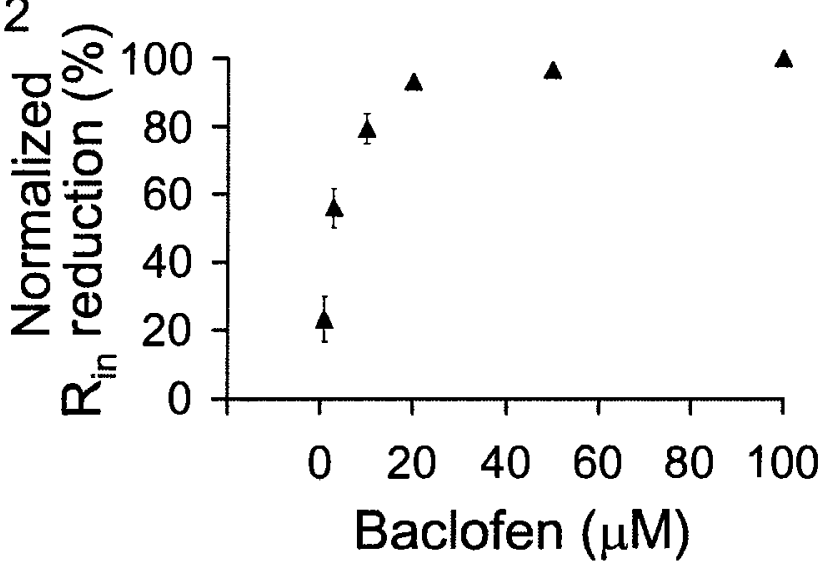

Figure 4.2. 


\section{Figure 4.2. Baclofen affected the resting potential and input resistance of ICD} neurons in a concentration-dependent manner. $A_{1}$ : Changes in the resting potential of a neuron before, during and after application of baclofen of 3,20 and $100 \mu \mathrm{M}$. Each point represents a measurement that was taken every $15 \mathrm{sec}$. Note that the resting potential became more hyperpolarized as concentration of baclofen increased. $\boldsymbol{A}_{2}$, The membrane hyperpolarization to baclofen of a given concentration was normalized as a percentage of the maximum hyperpolarization to baclofen (usually $50-100 \mu \mathrm{M}$ ) and then was plotted as a function of the concentration of baclofen. The data point represents the average and vertical bar represents S.E.M. $(\mathrm{n}=5)$. $\boldsymbol{B}_{1}$ : Responses of the same neuron shown in $\boldsymbol{A}$ to a hyperpolarizing current pulse of $80 \mathrm{pA}$ in ACSF, and baclofen of 3, 20 and $100 \mu \mathrm{M}$. Note that the membrane input resistance became smaller as the concentration of baclofen increased. $\boldsymbol{B}_{2}$ : The reduction in the input resistance was normalized as a percentage of the maximum reduction to baclofen of $50-100 \mu \mathrm{M}$ and then was plotted as a function of the concentration of baclofen. The data point represents the average and vertical bar represents S.E.M. $(n=5)$. 

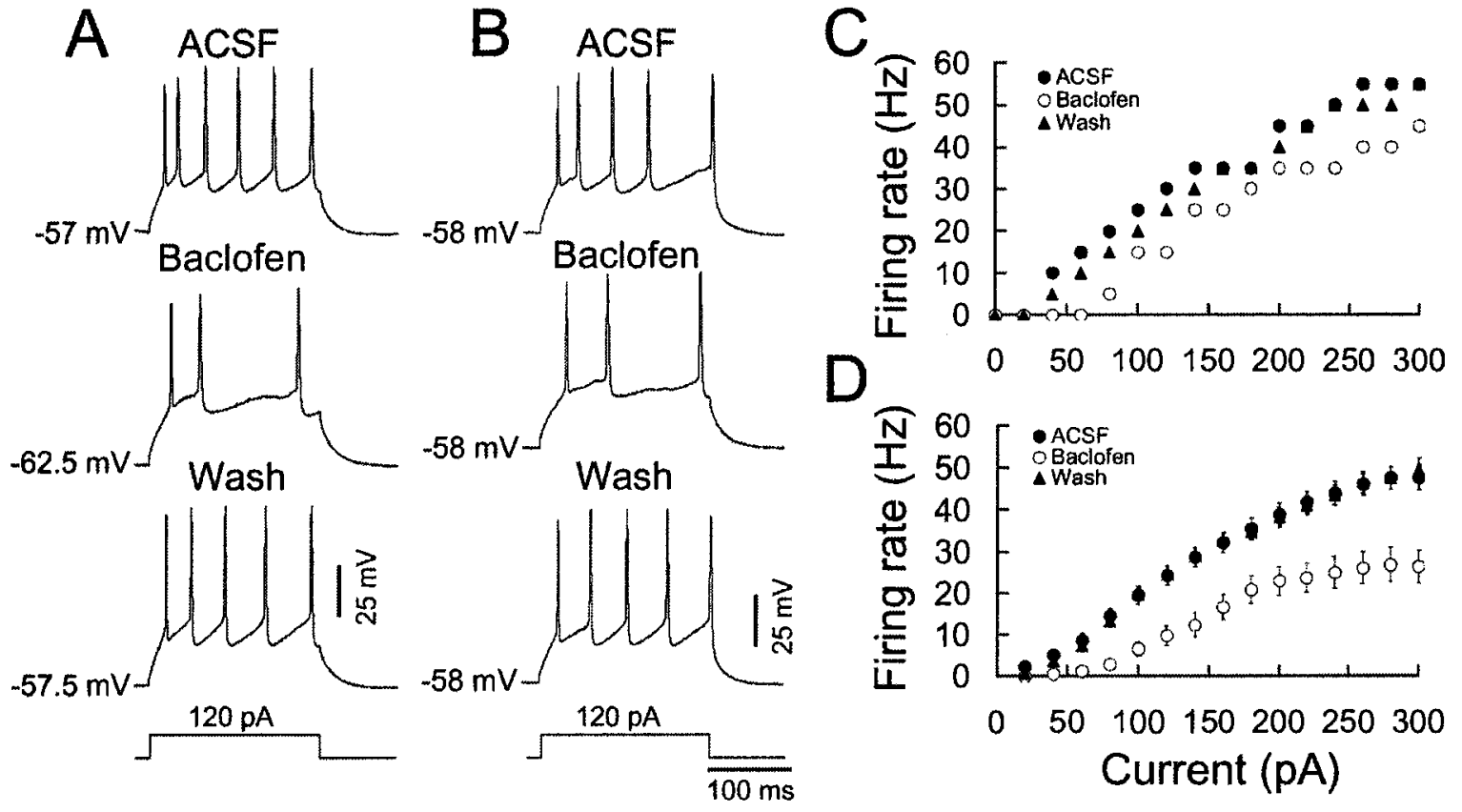

Figure 4.3. 
Figure 4.3. Baclofen depressed the membrane excitability of ICD neurons. A: Responses of an ICD neuron to a 120 pA depolarizing current injection ( $200 \mathrm{~ms}$ duration) before (upper trace), during (middle trace), and after (bottom trace) application of $20 \mu \mathrm{M}$ baclofen. Note the number of action potentials decreased and the membrane was hyperploarized from $-57 \mathrm{mV}$ to $-62.5 \mathrm{mV}$ during baclofen application. B: Responses to a $120 \mathrm{pA}$ depolarizing current injection (200 ms duration) before (upper trace), during (middle trace), and after (bottom trace) $20 \mu \mathrm{M}$ baclofen application in the same neuron as shown in $\boldsymbol{A}$. During baclofen application the membrane potential was adjusted to the original resting level with DC current. Note the similar reduction in the number of spikes as illustrated in $\boldsymbol{A}$. C: Firing frequency was plotted as a function of the current level before ( $\bullet$ ), during (०), and after ( $\boldsymbol{\Delta}$ ) baclofen application in the same neuron shown in $\boldsymbol{A}$ and $\boldsymbol{B}$. D: The average firing frequency of 37 ICD neurons was plotted as a function of the current level before $(\bullet)$, during $(0)$, and after $(\boldsymbol{\Delta})$ baclofen application. 


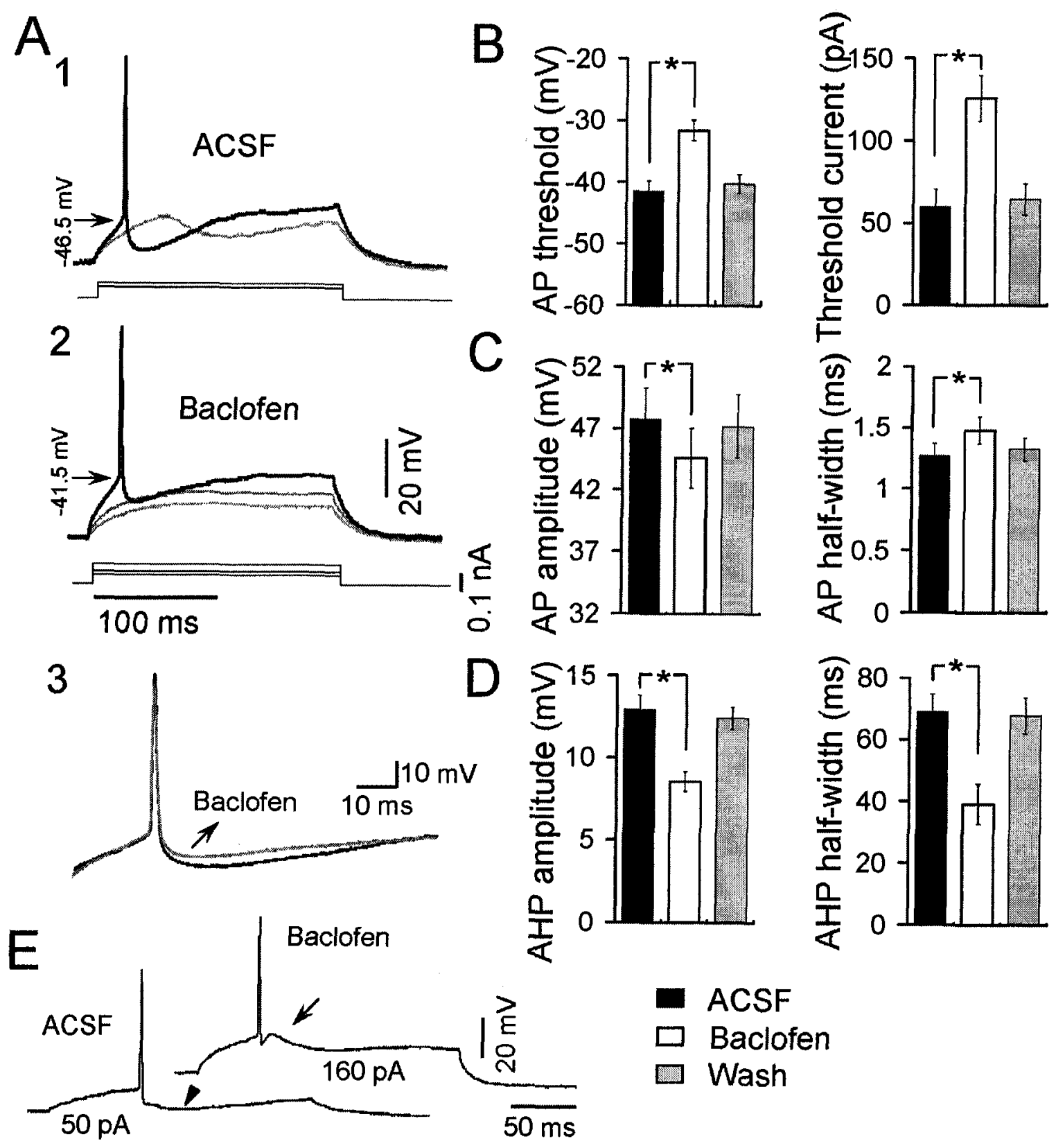

Figure 4.4. 
Figure 4.4. Baclofen modulated action potential properties in ICD neurons. A: Baclofen increased the threshold of the action potential and reduced the AHP in an ICD neuron. $\mathrm{A}_{1}$ : Responses to $60 \mathrm{pA}$ (grey trace) and $80 \mathrm{pA}$ (black trace) positive current injection (200 ms duration) before application of $20 \mu \mathrm{M}$ Baclofen. Note that $80 \mathrm{pA}$ current injection evoked an action potential with a threshold of $-46.5 \mathrm{mV}$. $\mathbf{A}_{2}$ : Responses to 60 and $80 \mathrm{pA}$ (grey traces) and $120 \mathrm{pA}$ (black trace) current injections (200 ms duration) during application of $20 \mu \mathrm{M}$ baclofen. Note that action potential could not be evoked until the current injection level increased to $120 \mathrm{pA}$. The action potential had a threshold of $-41.5 \mathrm{mV}$ during baclofen application. $\mathbf{A}_{3}$ : Same action potential waveforms in ACSF and baclofen as shown in $\boldsymbol{A}_{1-2}$ were overlapped and displayed at an expanded time scale. Note that the amplitude of both action potential and AHP was decreased in the presence of baclofen (grey trace). B-D: Comparisons of the average action potential (AP) threshold (B left panel), threshold current (B right panel), AP amplitude ( $\boldsymbol{C}$ left panel), AP half-width ( $\boldsymbol{C}$ right panel), AHP amplitude (D left panel) and AHP half-width (D right panel) from 28 ICD neurons before (black column), during (white column) and after (grey column) $20 \mu \mathrm{M}$ baclofen application $\left({ }^{*} P<0.05\right)$. E: Another example of baclofeninduced modulation on AHP. Note that the slow AHP was greatly compressed during application of $20 \mu \mathrm{M}$ baclofen (arrow). 


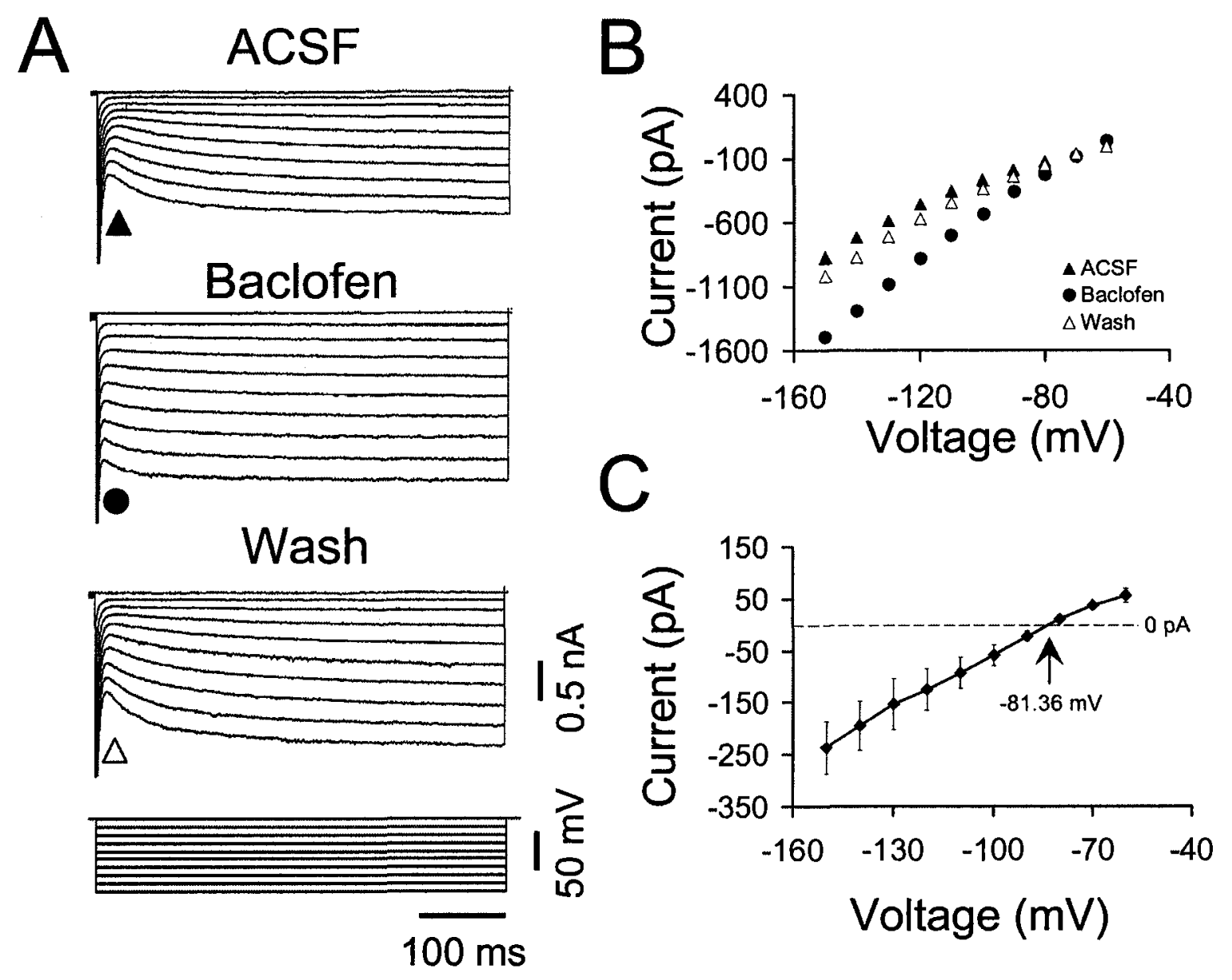

Figure 4.5. 
Figure 4.5. Baclofen evoked an inward rectifying $\mathrm{K}^{+}$current in ICD neurons. A: Responses to a series of voltage steps (from -60 to $-150 \mathrm{mV}$ by $-10 \mathrm{mV}$ increment, 500 ms duration) before (top traces), during (middle traces), and after (bottom traces) application of $20 \mu \mathrm{M}$ baclofen in an ICD neuron under voltage clamp mode. B: I-V

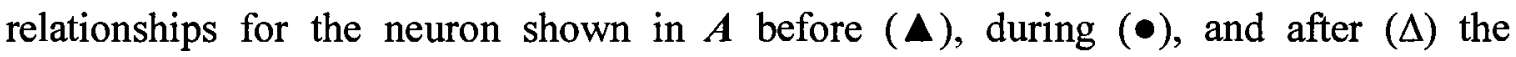
application of $20 \mu \mathrm{M}$ baclofen. The measurements of current amplitude were taken at indicated points in $\boldsymbol{A}$. C: The mean net baclofen-induced current was determined by substracting the currents before baclofen application from those after baclofen $(20 \mu \mathrm{M})$ application in 16 ICD neurons. The I-V curve for the baclofen-induced current was approximately linear and reversed at $-81.36 \mathrm{mV}$ (arrow). The dash line represents the baseline level $(0 \mathrm{pA})$. 


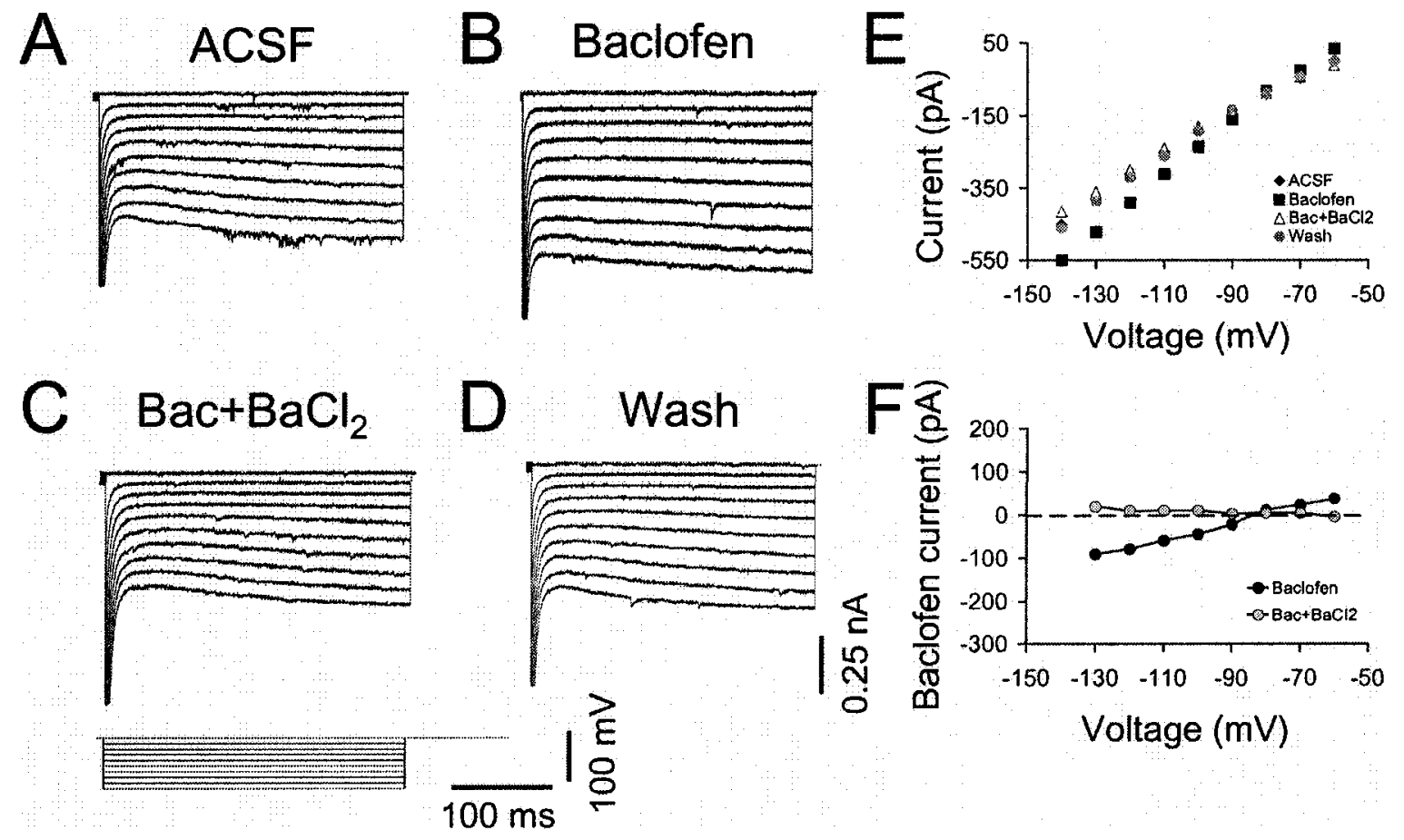

Figure 4.6. 
Figure 4.6. Baclofen-induced current was blocked by barium. A-D: Responses to a series of voltage steps (from -60 to $-130 \mathrm{mV}$ in $-10 \mathrm{mV}$ steps, $300 \mathrm{~ms}$ duration) from an ICD neuron in $\operatorname{ACSF}(\boldsymbol{A}), 20 \mu \mathrm{M}$ baclofen $(\boldsymbol{B}), 20 \mu \mathrm{M}$ baclofen plus $1 \mathrm{mM} \mathrm{BaCl}_{2}(\boldsymbol{C})$, and ACSF wash $(\boldsymbol{D})$. Note that barium attenuated the effect of baclofen. E: I-V plots for the four conditions shown in A-D. F: Plot of baclofen-induced net current from the neuron shown in $\boldsymbol{A}-\boldsymbol{D}$ during baclofen (•) and (०) baclofen with $\mathrm{BaCl}_{2}$. 


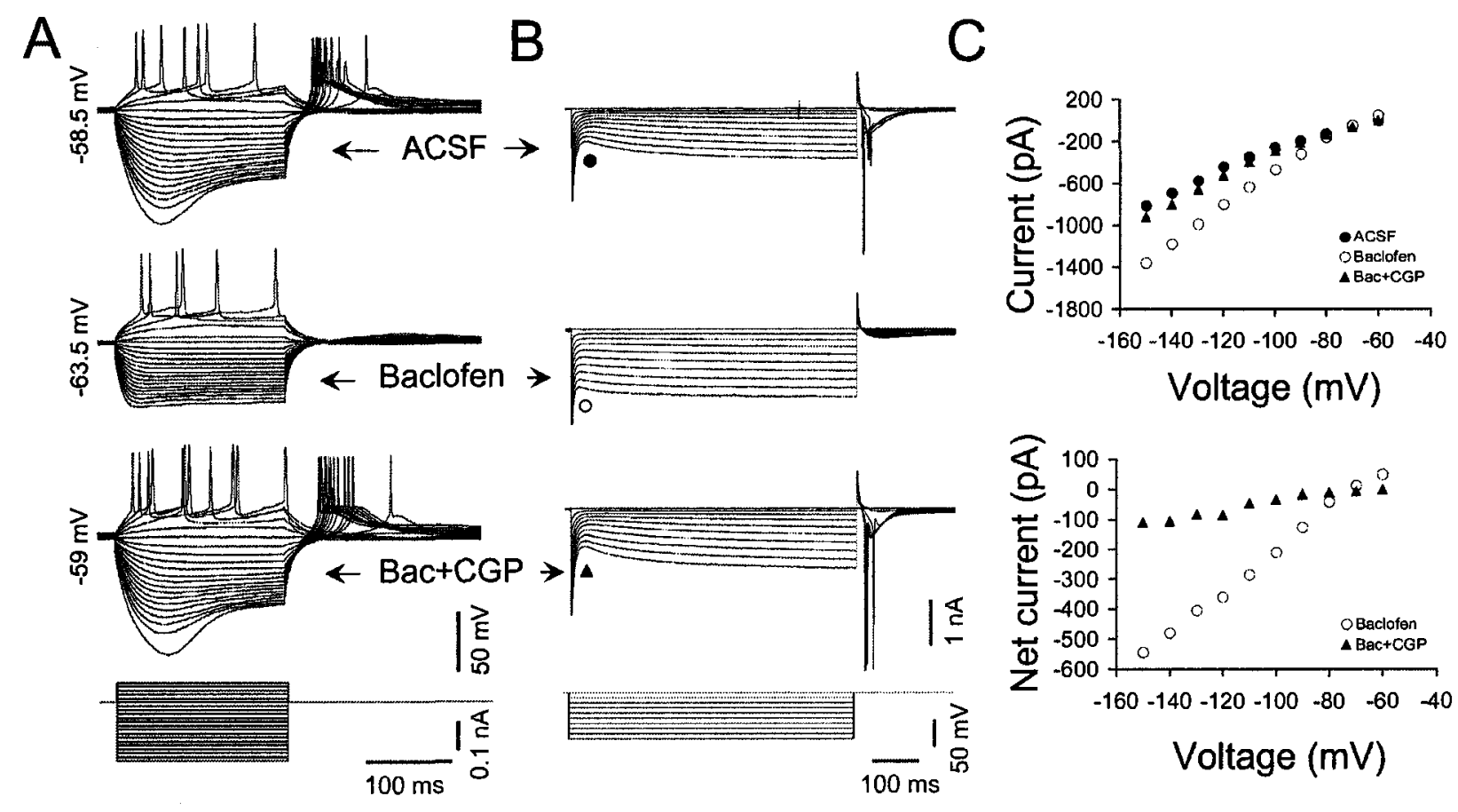

Figure 4.7. 
Figure 4.7. CGP35348 blocked the effects of baclofen in ICD neurons. A: Responses to a series of current steps from -300 to $100 \mathrm{pA}$ ( $20 \mathrm{pA} / \mathrm{step}, 200 \mathrm{~ms}$ duration) in an ICD neuron under the conditions of ACSF (upper traces), $20 \mu \mathrm{M}$ baclofen (middle traces), and co-application of $100 \mu \mathrm{M}$ CGP35348 with $20 \mu \mathrm{M}$ baclofen (bottom traces). Note that CGP35348 reversed the effects of baclofen on the membrane potential, input resistance, firing and rebound depolarization. B: Responses to a series of voltage steps from -60 to $-150 \mathrm{mV}(10 \mathrm{mV} / \mathrm{step}, 500 \mathrm{~ms}$ duration) in another ICD neuron when the slice was perfused with ACSF (upper traces), $20 \mu \mathrm{M}$ baclofen (middle traces), and 100 $\mu \mathrm{M}$ CGP35348 plus $20 \mu \mathrm{M}$ baclofen (bottom traces). The symbols indicate the point where the measurements were made. C: Upper panel: I-V relationship of the ICD neuron shown in $B$ under the conditions of ACSF (•), $20 \mu \mathrm{M}$ baclofen (o), and $100 \mu \mathrm{M}$ CGP35348 plus $20 \mu \mathrm{M}$ baclofen ( $\boldsymbol{\Delta})$. Lower panel: The baclofen-induced current in the same neuron as shown in $\boldsymbol{B}$ where baclofen $(20 \mu \mathrm{M})$ was applied $(0)$ and baclofen was co-administered with $100 \mu \mathrm{M}$ CGP35348 ( $\mathbf{\Delta}$ ). Note that CGP35348 nearly abolished the baclofen-induced current. 


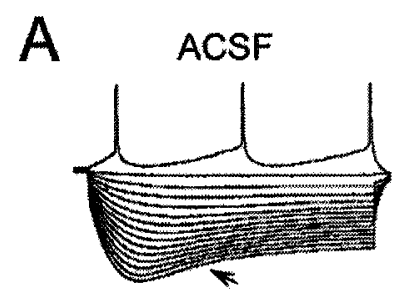

207288

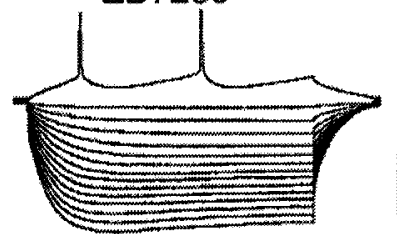

- $\frac{1}{50 \mathrm{~ms}}$
B ACSF

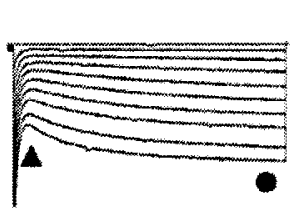

ZD7288

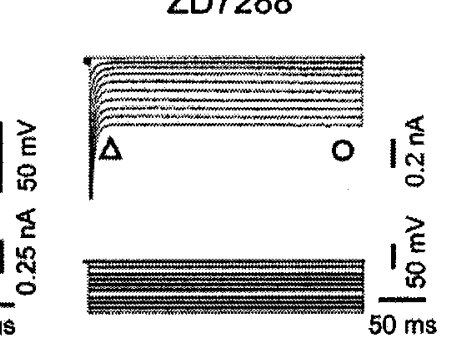

C
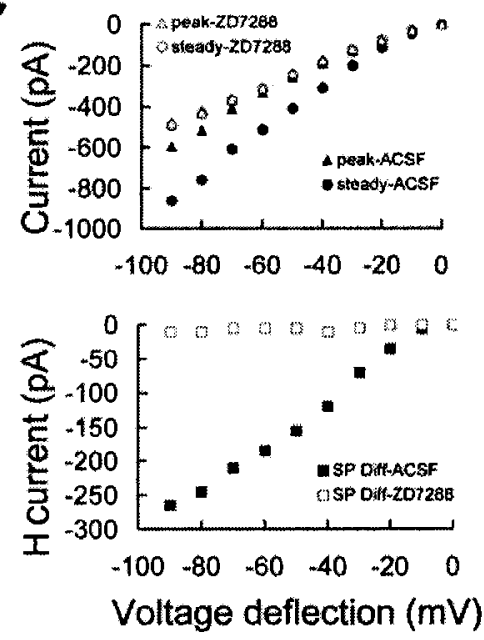

D
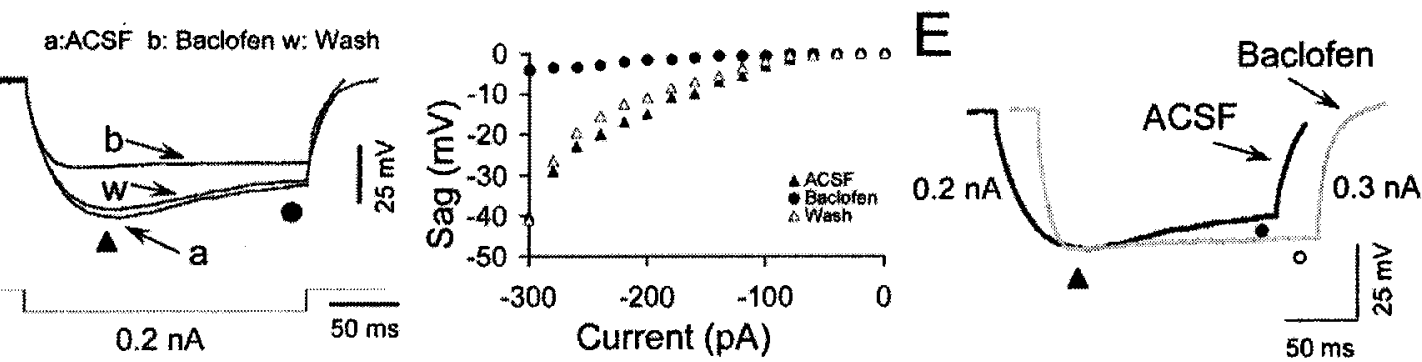

F
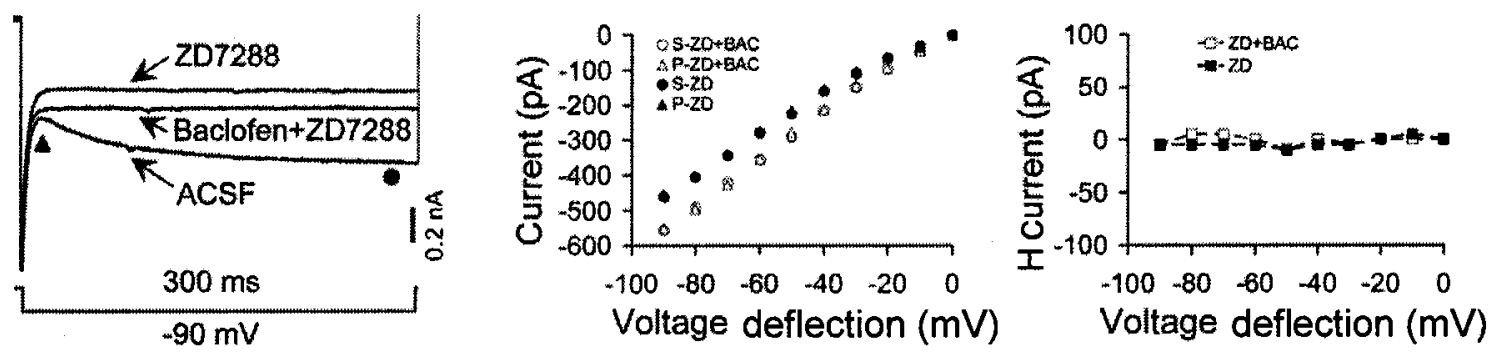

Figure 4.8. 
Figure 4.8. Baclofen suppressed $I_{h}$ currents in ICD neurons. A: Responses to depolarizing and hyperpolarizing current steps ( -300 to $40 \mathrm{pA})$ of an ICD neuron before (upper traces) and during (lower traces) application of $100 \mathrm{nM}$ ZD7288. Note that a large depolarizing sag (arrow) emerged with larger negative current injections. The sag was abolished by ZD7288. B: Responses to a series of voltage steps (-60 to $-150 \mathrm{mV}$ ) of the same ICD neuron as shown in $\boldsymbol{A}$ before (upper traces) and during (lower traces) application of $100 \mathrm{nM}$ ZD7288. The instantaneous current was measured at the point indicated by " $\Delta$ " and " $\Delta$ ", while the steady current was measured at the point indicated by "๑" and "O". Note that ZD7288 eliminated the difference between the instantaneous current and the steady current, which was $\mathrm{I}_{h}$. C: Upper panel, instantaneous and steady currents were plotted as a function of voltage steps before and during application of ZD7288. Lower panel: The $\mathrm{I}_{h}$ was plotted as a function of voltage steps before $(\mathbf{E})$ and during (ם) ZD7288 application. D: Left panel, responses to a hyperpolarizing current step $(-200 \mathrm{pA})$ of an ICD neuron before, during and after application of $20 \mu \mathrm{M}$ baclofen. The membrane potential sag was measured as the difference between the peak $(\boldsymbol{\Delta})$ and steady $(\bullet)$ voltage changes. Right panel, the amplitude of the sag was plotted as a function of current steps before $(\boldsymbol{\Delta})$, during $(\bullet)$ and after $(\Delta)$ baclofen application. $\mathbf{E}$ : Responses to $200 \mathrm{pA}$ (in ACSF, black trace) and -300 pA (in baclofen, grey trace) of the same neuron as shown in $\boldsymbol{D}$. Note that the peak voltage changes $(\boldsymbol{\Delta})$ were the same under both conditions, while the steady voltage changes ( $\bullet$, in ACSF; $\circ$, in baclofen) were different. F: Responses of an ICD neuron to a voltage step ( $-90 \mathrm{mV}, 300 \mathrm{~ms}$ duration) under those conditions (ACSF, ZD7288, baclofen plus ZD7288) (left panel). The instantaneous current, steady current $I_{h}$ were plotted as a function of voltage steps in ZD7288 and baclofen plus ZD7288 solution (middle and right panels). 


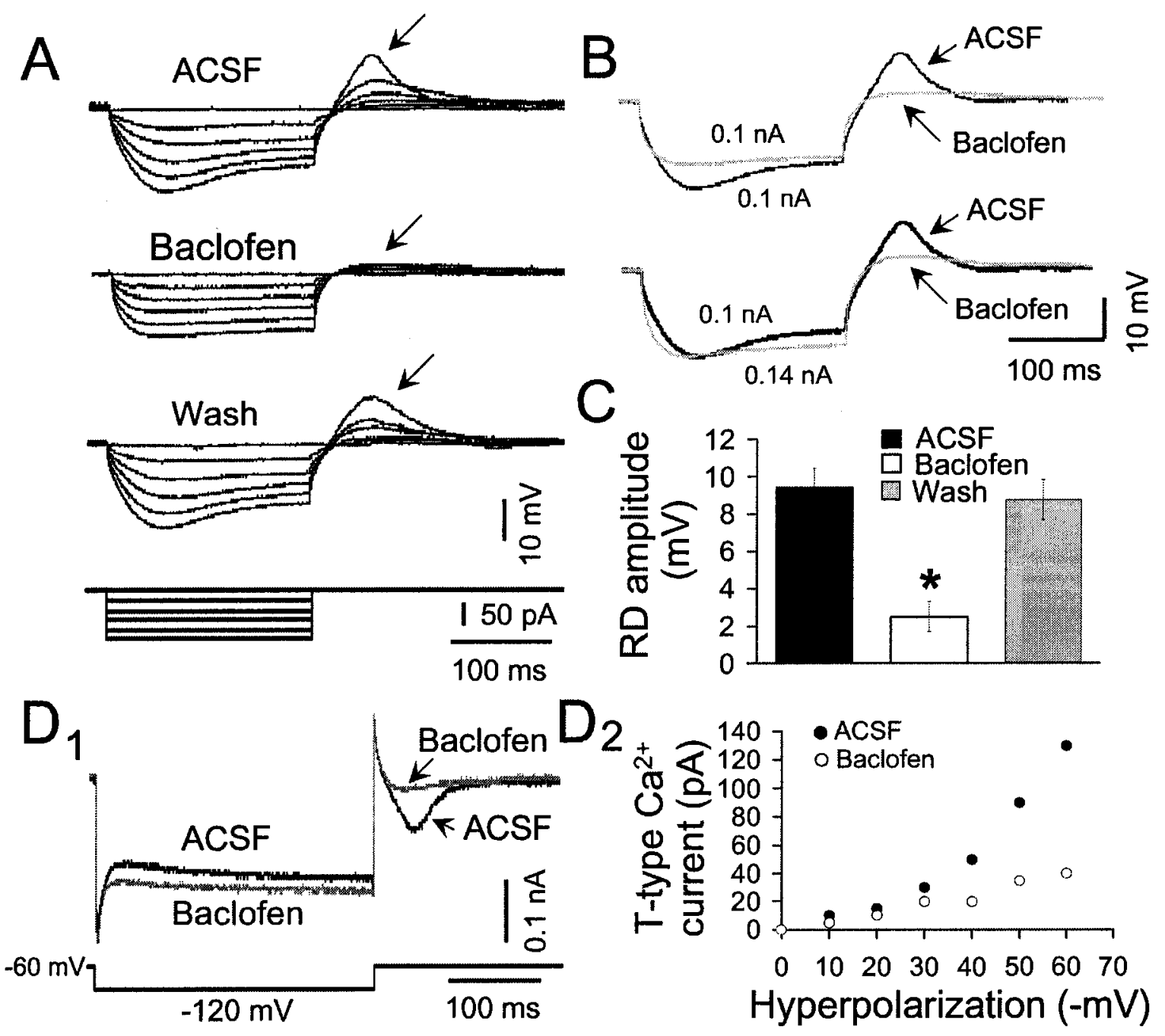

Figure 4.9. 


\section{Figure 4.9. Baclofen suppressed $\mathrm{T}$-type $\mathrm{Ca}^{2+}$ mediated rebound depolarization in}

ICD neurons. A: Responses to a series of hyperpolarizing current steps from 0 to -100 $\mathrm{pA}(-20 \mathrm{pA} / \mathrm{step}, 200 \mathrm{~ms}$ duration) in an ICD neuron before (top traces), during (middle traces), and after (bottom traces) application of baclofen $(20 \mu \mathrm{M})$. Note that the rebound depolarization $(\mathrm{RD}$, arrow) generated upon the release of the hyperpolarizing current was depressed during application of baclofen. B: Upper traces: responses to $-0.1 \mathrm{nA}$ current injection under the conditions of ACSF (black trace) and $20 \mu \mathrm{M}$ baclofen (grey trace). Lower traces: a response to $-0.1 \mathrm{nA}$ current injection with the slice in ACSF (black trace) and a response to $-0.14 \mathrm{nA}$ current injection with the slice in $20 \mathrm{mM}$ baclofen (grey trace). Note that the intensity of the current was adjusted to induce the similar peak voltage change in baclofen as that in ACSF, however, the RD was still not elicited during baclofen application. $\mathbf{C}$ : The average $\mathrm{RD}$ amplitude was significant reduced during application of baclofen $\left({ }^{*} P<0.001, \mathrm{n}=12\right)$. $\mathbf{D}_{1}$ : Responses to a voltage step of $-120 \mathrm{mV}$ (-60 $\mathrm{mV}$ holding level) in an ICD neuron before (black trace) and during (grey trace) application of $20 \mu \mathrm{M}$ baclofen. Note that an inward current (T-type $\mathrm{Ca}^{2+}$ current, arrow) activated upon the release of the voltage step was abolished by application of baclofen. $\mathbf{D}_{2}$ : The amplitude of $\mathrm{T}$-type $\mathrm{Ca}^{2+}$ current was plotted as a function of prehyperpolarization in the same neuron as shown in $\boldsymbol{D}_{\boldsymbol{l}}$ before $(\bullet)$ and during $(\circ)$ application of baclofen. 


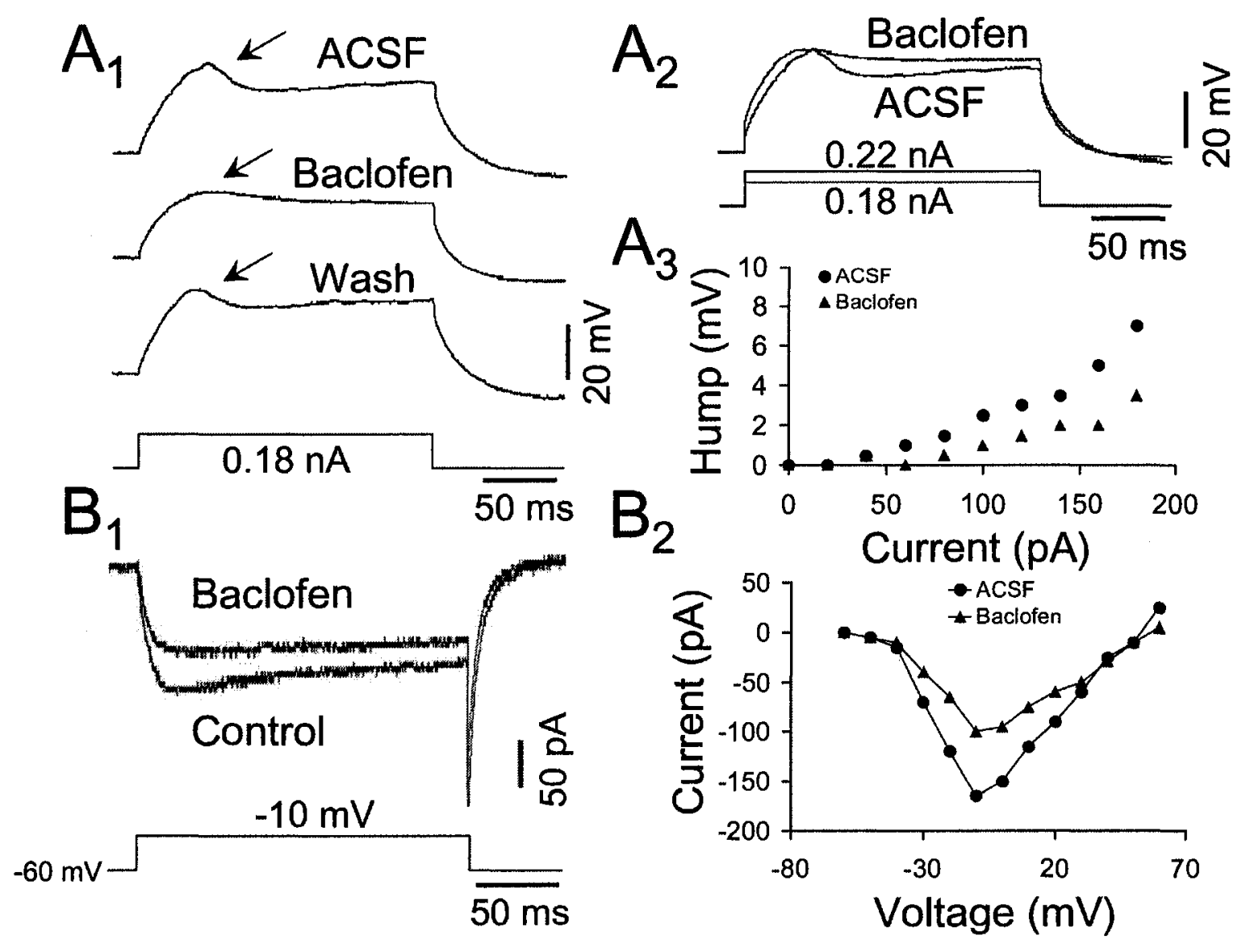

Figure 4.10. 


\section{Figure 4.10. Baclofen attenuated the high-threshold $\mathrm{Ca}^{2+}$ currents in ICD neurons.}

A: Baclofen depressed the depolarizing hump in an ICD neuron. $\mathbf{A}_{\mathbf{1}}$ : Membrane voltage responses to a depolarizing current step of $180 \mathrm{pA}$ ( $200 \mathrm{~ms}$ duration) before (top trace), during (middle trace), and after (bottom trace) application of $20 \mu \mathrm{M}$ baclofen. Note that the depolarizing hump was depressed during application of baclofen. $\mathbf{A}_{\mathbf{2}}$ : Responses to $\mathrm{A}$ $0.18 \mathrm{nA}$ current pulse in ACSF and to a $0.22 \mathrm{nA}$ current pulse in $20 \mathrm{mM}$ baclofen solution. Note that the intensity of the current was adjusted to induce the similar voltage response in baclofen as that in ACSF, however, the obvious hump still did not appear. $\mathbf{A}_{\mathbf{3}}$ : The amplitude of the hump was plotted as a function of the current steps in the neuron as

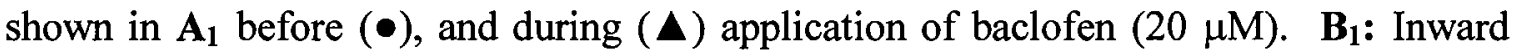
$\mathrm{Ca}^{2+}$ currents of an ICD neuron were recorded in response to a voltage step from -60 to $10 \mathrm{mV}$ (150 ms duration) with the brain slice in a control ACSF containing TTX $(1 \mu \mathrm{M})$, TEA $(20 \mu \mathrm{M})$ and 4-AP $(2 \mathrm{mM})$, and after administering baclofen $(20 \mu \mathrm{M})$ to the control ACSF. $\mathbf{B}_{2}$ : The $\mathrm{Ca}^{2+}$ currents during control $\operatorname{ACSF}(\bullet)$ and after application of baclofen ( $\boldsymbol{A}$ ) for the same neuron as shown in $\boldsymbol{B}$ were plotted as a function of voltage steps. 


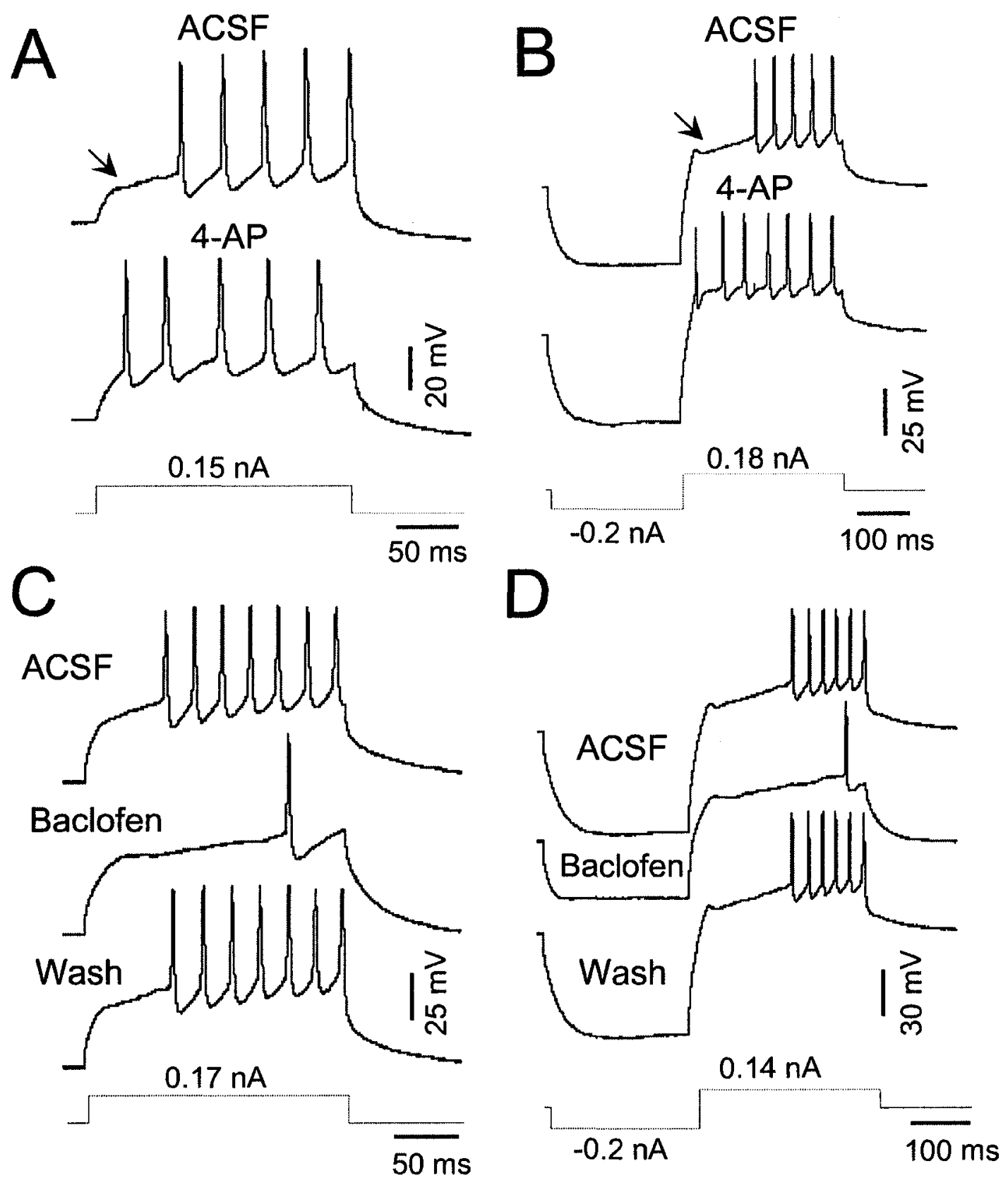

Figure 4.11. 
Figure 4.11. Baclofen prolonged the delay in firing in ICD neurons. A: Action potentials evoked by a $150 \mathrm{pA}$ depolarizing current step before (top trace) and during (bottom trace) application of $1 \mathrm{mM}$ 4-AP in an ICD neuron with a buildup firing pattern. Note that the delay of firing in control ACSF (arrow) was abolished by application of 4AP. B: The delay in firing (arrow) was only appeared when prehyperpolarization (-200 $\mathrm{pA}, 250 \mathrm{~ms}$ duration) was applied in another ICD neuron (top trace). The delay disappeared during application of $1 \mathrm{mM} 4-\mathrm{AP}$ (bottom trace). C: The delay in firing in response to a $170 \mathrm{pA}$ depolarizing current step (200 ms duration) in the neuron shown in $\boldsymbol{A}$ before (top trace), during (middle trace), and after (bottom trace) application of $20 \mu \mathrm{M}$ baclofen. D: The delay in firing in response to a $140 \mathrm{pA}$ depolarizing current step (200 ms duration) with a preceding $-200 \mathrm{pA}$ current step in the neuron shown in $\boldsymbol{B}$ before (top trace), during (middle trace), and after (bottom trace) application of $20 \mu \mathrm{M}$ baclofen. Note that baclofen increases the duration of the delay in firing in both cells. 

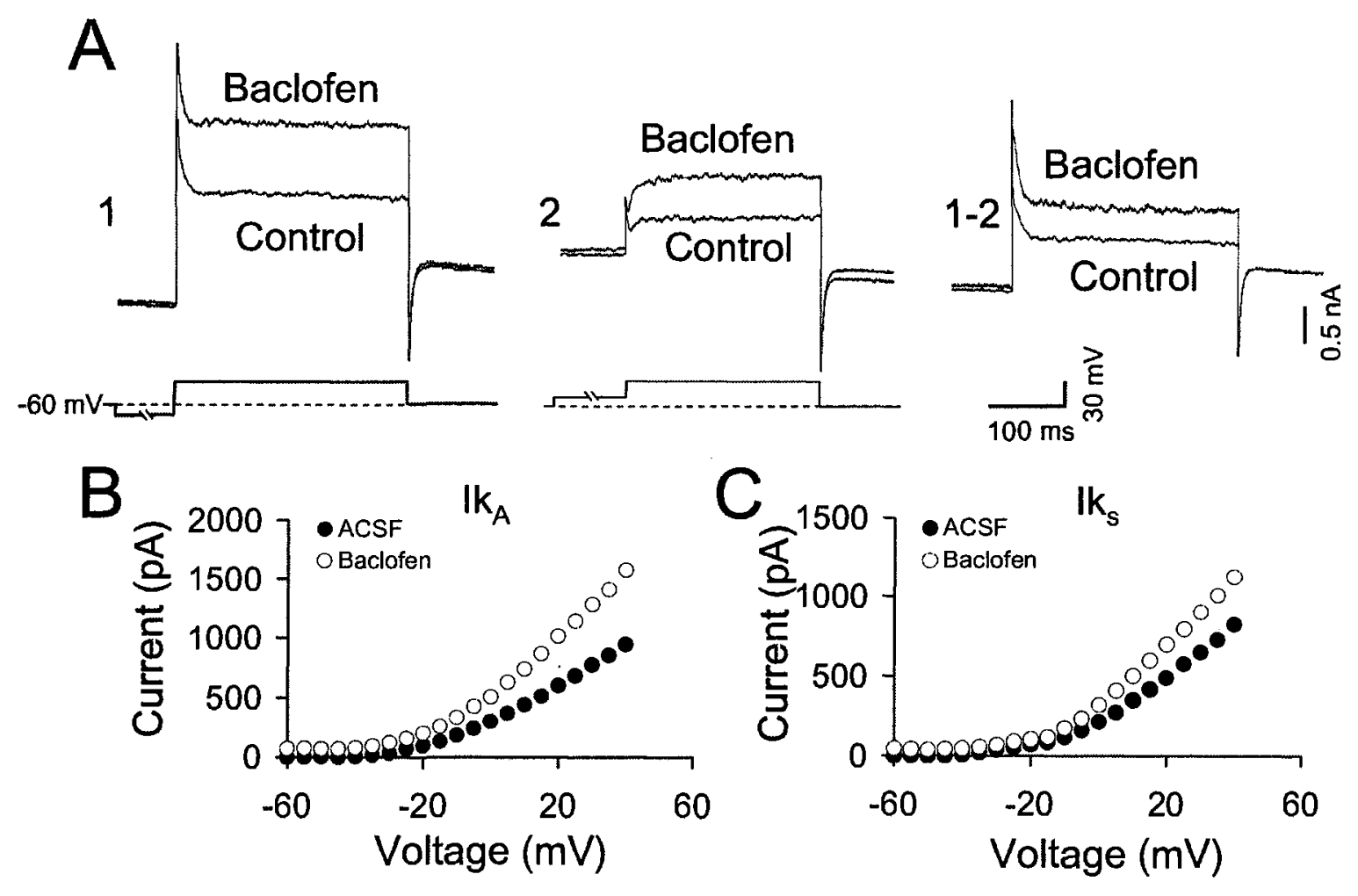

Figure 4.12. 
Figure 4.12. Baclofen enhances A-type and sustained voltage-dependent $\mathbf{K}^{+}$ currents in an ICD neuron. Whole-cell voltage clamp recordings were performed with a holding potential of $-60 \mathrm{mV}$. $\mathbf{A}_{1}$ : Outward currents were recorded in response to a voltage step from $-100 \mathrm{mV}$ (500 ms duration) to $30 \mathrm{mV}$ (300 ms duration) with the brain slice in ACSF containing TTX $(1 \mu \mathrm{M})$ and low $\mathrm{Ca}^{2+}$ concentration $(0.1 \mathrm{mM})$ (Control $\mathrm{ACSF}$ ), and in the control ACSF containing $20 \mu \mathrm{M}$ baclofen. $\mathbf{A}_{2}$ : Outward currents were recorded in response to a voltage step from $-40 \mathrm{mV}$ (500 ms duration) to $30 \mathrm{mV}$ (300 ms duration) with the brain slice in the control ACSF containing TTX $(1 \mu \mathrm{M})$ and low $\mathrm{Ca}^{2+}$ concentration $(0.1 \mathrm{mM})$ and in the control ACSF containing $20 \mu \mathrm{M}$ baclofen. A $\mathbf{A}_{1-2}$ : Atype $\mathrm{K}^{+}$currents in the control ACSF and in the control ACSF containing baclofen were obtained by subtracting the currents shown in $\boldsymbol{A}_{2}$ from those in $\boldsymbol{A}_{\boldsymbol{1}}$. B: A-type $\mathrm{K}^{+}$currents were plotted as a function of the voltage levels before $(\bullet)$ and during $(\odot)$ application of $20 \mathrm{mM}$ baclofen. C: Sustained $\mathrm{K}^{+}$currents were plotted as a function of the voltage levels before (•) and during (०) application of $20 \mathrm{mM}$ baclofen. 


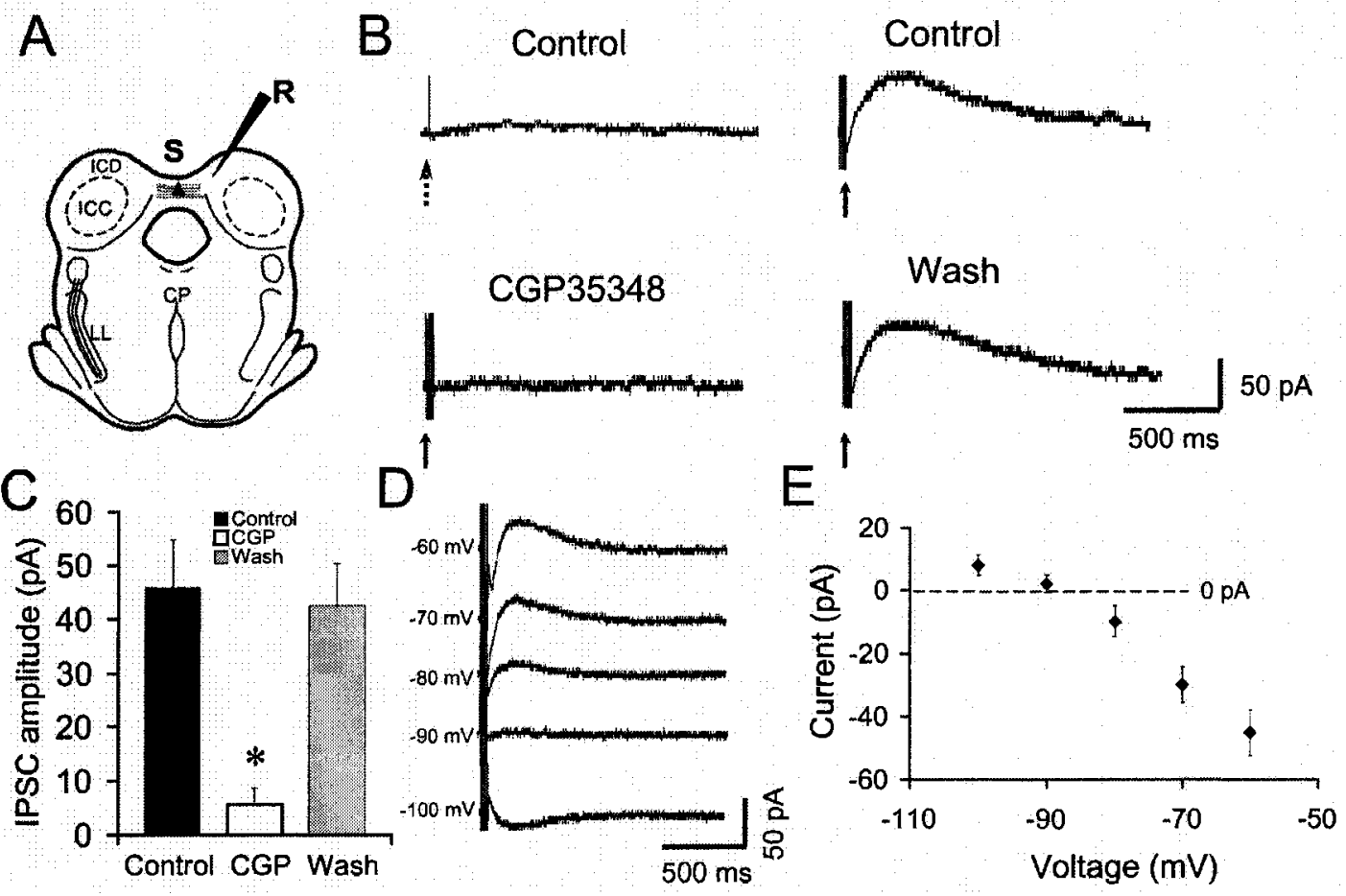

Figure 4.13. 
Figure 4.13. GABA B $_{B}$ receptor mediated IPSCs in ICD neurons. A: Schematic diagram of an IC brain slice. $S$ : the site for stimulation. $R$ : the site for recording. B: An example of slow IPSCs in an ICD neuron. Upper and left trace: Stimulation with a single pulse to the commissure of the IC did not elicit any significant response with the brain slice in CNQX $(10 \mu \mathrm{M})$, APV $(100 \mu \mathrm{M})$, bicuculline $(10 \mu \mathrm{M})$ and strychnine $(0.5 \mu \mathrm{M})$. Upper and right trace: Repetitive stimulation of 10 pulses at $200 \mathrm{~Hz}$ with the same intensity and location as the single pulse stimulation evoked a slow and long lasting outward current. Lower traces: The response was completely and reversibly blocked by the $\mathrm{GABA}_{\mathrm{B}}$ receptor antagonist, CGP35348 $(100 \mu \mathrm{M})$. C: The average amplitude of $\mathrm{GABA}_{\mathrm{B}}$ receptor mediated IPSCs before, during and after application of $100 \mu \mathrm{M}$ CGP35348 in ICD neurons $(* P<0.001, \mathrm{n}=5)$. D: IPSCs were recorded at five different holding potentials in the presence of CNQX $(10 \mu \mathrm{M})$, APV $(100 \mu \mathrm{M})$, bicuculline (10 $\mu \mathrm{M})$ and strychnine $(0.5 \mu \mathrm{M})$. Note that the polarity of the slow IPSCs reversed at a membrane potential of approximately $-90 \mathrm{mV}$. E: I-V plot of the slow IPSCs from 5 ICD neurons indicating a reversal potential of the IPSC of about $-90 \mathrm{mV}$. The dash line represents the baseline level $(0 \mathrm{pA})$. 

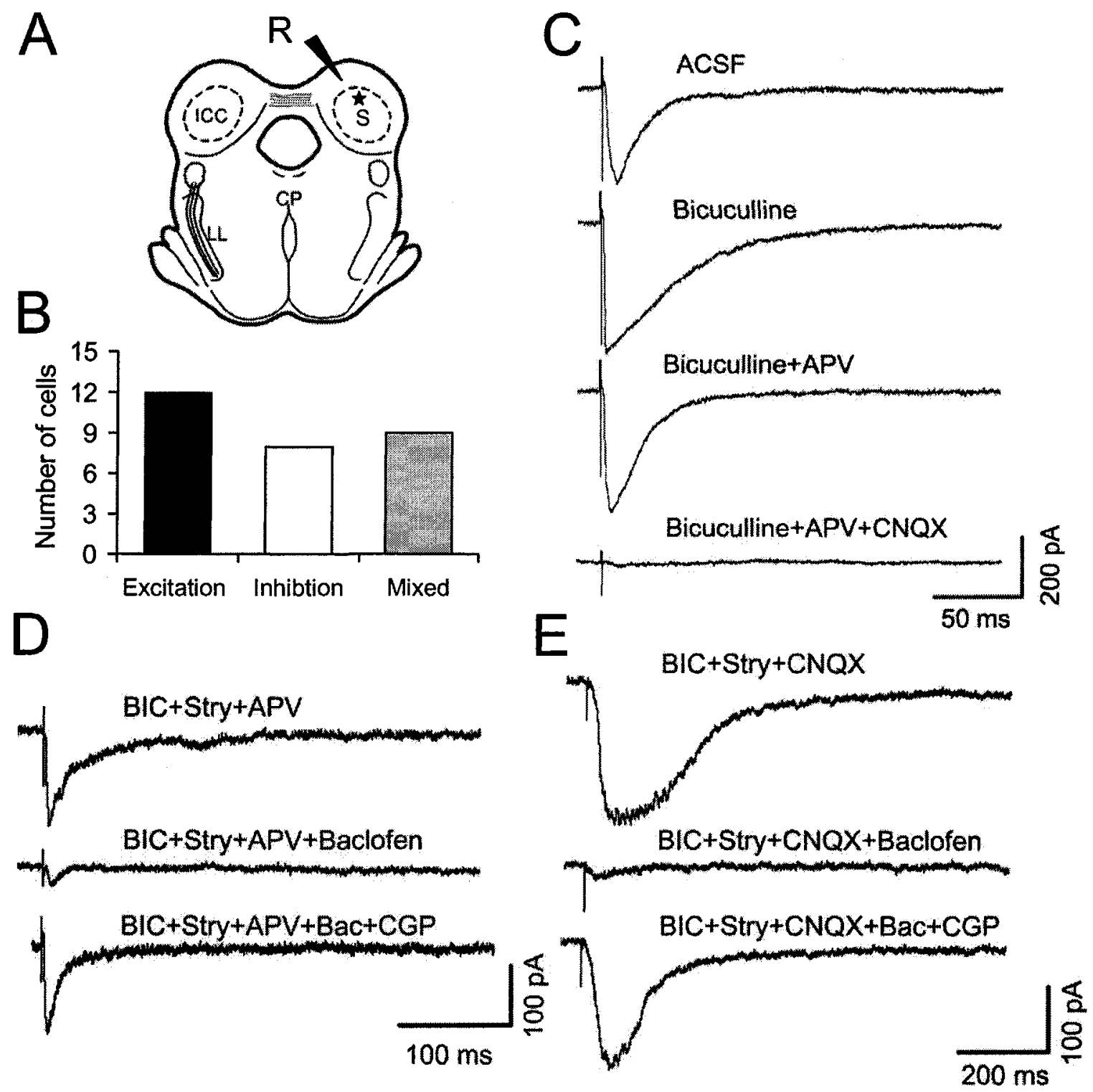

Figure 4.14. 


\section{Figure 4.14. Baclofen modulated excitatory synaptic transmission from ICC to ICD.}

A: Schematic diagram of an IC brain slice. $\boldsymbol{S}$ : the site for stimulation. $\boldsymbol{R}$ : the site for recording. B: The chart summarizes the distribution of different type of synaptic currents across the ICD neurons, including predominantly excitatory responses $(n=12$, black column), predominantly inhibitory responses $(\mathrm{n}=8$, white column) and mixed responses $(\mathrm{n}=9$, grey column). C: Synaptic responses of an ICD neuron to stimulation of the ICC with single pulse were dissociated pharmacologically. Control response (EPSC, top trace) was recorded in normal ACSF. Application of bicuculline enlarged the EPSC (second trace). Application of APV removed the later part of the EPSC (third trace). CNQX application completely removed the remaining EPSC (bottom trace). Note pharmacological agents were cumulatively applied as shown in the Figure. D and E: Baclofen depressed the AMPA $(\boldsymbol{D})$ and NMDA $(\boldsymbol{E})$ EPSCs recorded from two separate ICD neurons. AMPA $(\boldsymbol{D})$ and NMDA $(\boldsymbol{E})$ EPSCs were pharmacologically isolated (top traces). Application of baclofen depressed both the AMPA $(\boldsymbol{D})$ and NMDA $(\boldsymbol{E})$ EPSCs (middle traces). Application of CGP35348 blocked the baclofen-induced suppression in AMPA and NMDA EPSCs (bottom traces). The vertical lines indicate the artifacts produced by the single stimulation. 
A
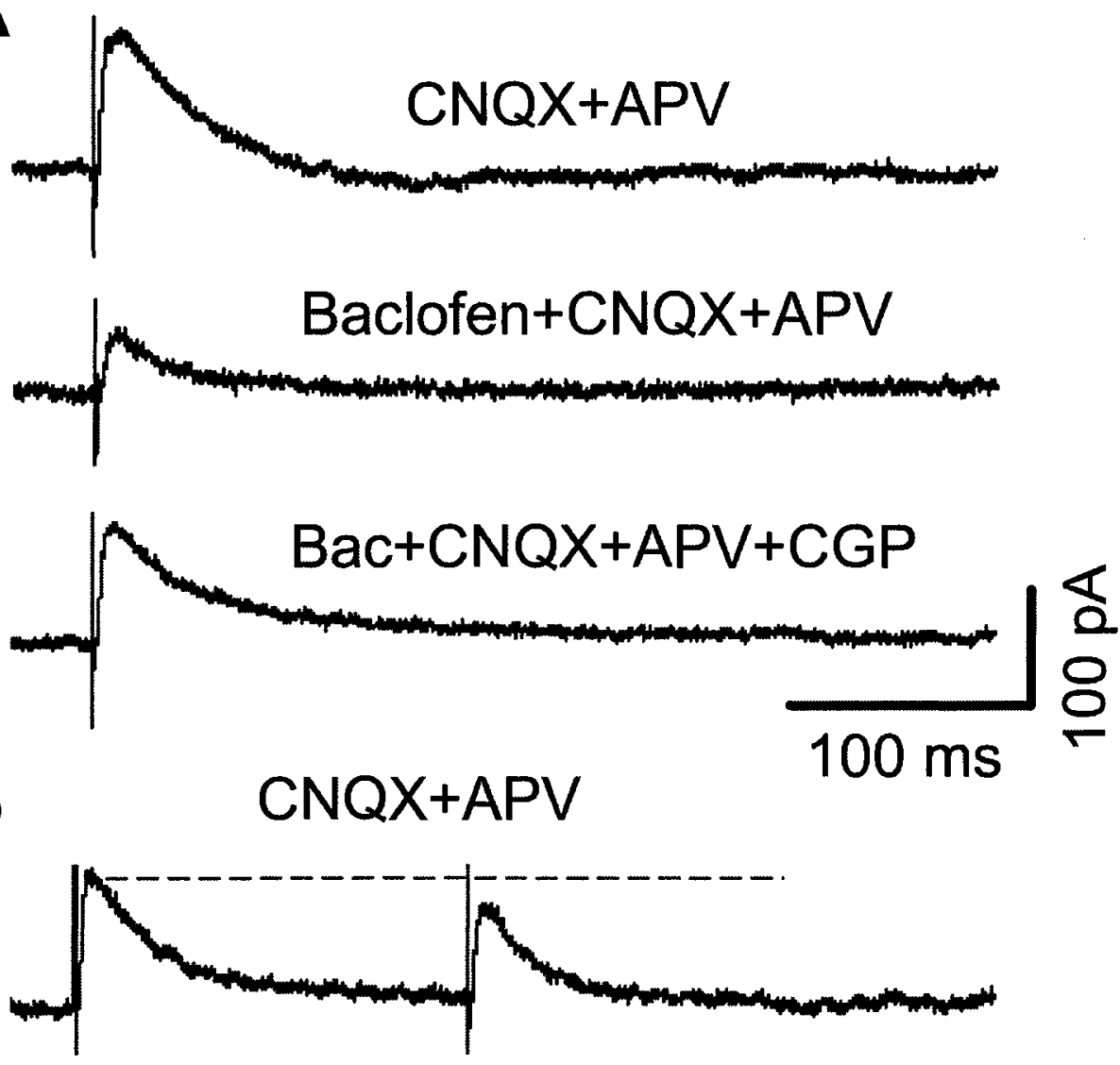

Baclofen+CNQX+APV

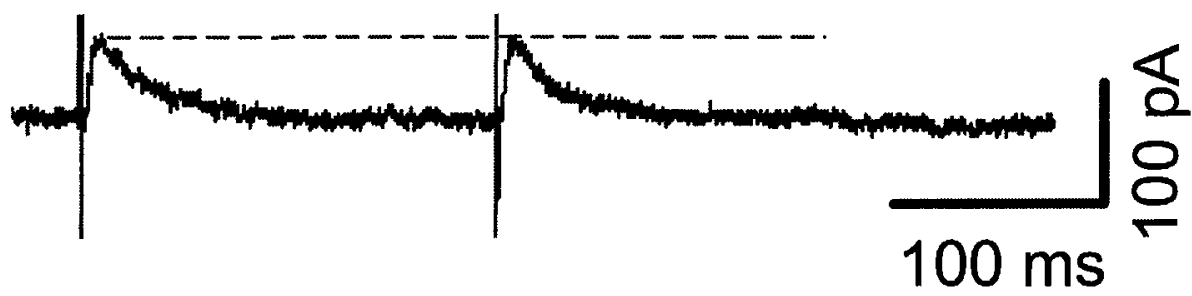

Figure 4.15. 
Figure 4.15. Baclofen suppressed IPSCs mediated by $\mathrm{GABA}_{\mathrm{A}}$ receptors in an ICD neuron. A: $A \mathrm{GABA}_{\mathrm{A}}$ receptor mediated IPSC was depressed by application of $5 \mu \mathrm{M}$ baclofen. Upper trace, $\mathrm{GABA}_{\mathrm{A}}$ receptor mediated IPSC was pharmacologically isolated by application of APV and CNQX. Middle trace, application of $5 \mu \mathrm{M}$ baclofen depressed the GABA $\mathrm{A}_{\mathrm{A}}$ IPSC. Bottom trace, application of $100 \mu \mathrm{M}$ CGP35348 decreased the suppression of IPSC caused by baclofen. B: Baclofen increased the PPR of GABA $_{A}$ receptor mediated IPSCs. Upper trace, $\mathrm{GABA}_{\mathrm{A}}$ IPSCs were recorded in APV and CNQX solution to a paired-pulse stimulation at an interval of 200 ms. Lower trace, Application of $5 \mu \mathrm{M}$ baclofen depressed the first IPSC more than the second IPSC. 


\subsection{Discussion}

My study using in vitro whole-cell patch clamp recording technique provides the first direct evidence that functional postsynaptic $\mathrm{GABA}_{\mathrm{B}}$ receptors are located on the membrane of ICD neurons. Activation of postsynaptic $\mathrm{GABA}_{B}$ receptors by the $\mathrm{GABA}_{\mathrm{B}}$ receptor agonist baclofen evoked a concentration-dependent inhibitory effect on the membrane excitability. The baclofen-evoked inhibition was mediated by several distinct ionic conductances, i.e., an inward rectifying potassium current, a transient A-current, an $\mathrm{I}_{h}$, a low-threshold $\mathrm{T}$ type calcium current, a high-threshold calcium current, and a calcium activated potassium current. In addition, both AMPA and NMDA receptor mediated excitatory and $\mathrm{GABA}_{\mathrm{A}}$ receptor mediated inhibitory synaptic transmission from the ICC to ICD can be depressed by activation of $\mathrm{GABA}_{B}$ receptors through a presynaptic mechanism.

\subsubsection{Postsynaptic $G A B A_{B}$ receptors in ICD neurons}

In previous anatomical studies, Fubara et al. (1996) reported that low-level binding for $\mathrm{GABA}_{\mathrm{B}}$ receptors relative to $\mathrm{GABA}_{\mathrm{A}}$ receptors was observed throughout the IC of the big brown bat with more concentrated binding in the dorsalmedial area which is thought to belong to the ICD. Consistent with this result are reports from the rat by autoradiographic techniques (Chu et al., 1990; Milbrandt et al., 1994). However, the anatomical data can not provide the information whether the $\mathrm{GABA}_{\mathrm{B}}$ receptors are preor postsynaptically located and what physiological roles they may play in auditory information processing. Results from previous electrophysiological studies have shown that activation of presynaptic $\mathrm{GABA}_{B}$ receptors in ICC neurons modulates both GABA 
and glutamate neurotransmitter release (Ma et al., 2002; Sun et al., 2006). However, baclofen application did not change membrane conductances of ICC neurons (Ma et al., 2002). Thus, it seems reasonable to propose the lack of postsynaptic $\mathrm{GABA}_{B}$ receptors on ICC neurons.

In the present study, I have observed that application of the $\mathrm{GABA}_{\mathrm{B}}$ receptor agonist, baclofen, caused a hyperpolarization in the majority of ICD neurons (40/57) and the response was associated with a decrease in the membrane input resistance. The baclofen-induced hyperpolarization and decreased input resistance were dose-dependent. Under voltage clamp mode, baclofen application induced an outward current that was blocked by barium. These results suggest activation of inward rectifier potassium channels by baclofen (Williams et al., 1988; Hille, 2001; Slugg et al., 2003; Takeda et al., 2004). This current may induce the decrease of the cell's resting potential (membrane hyperpolarization). Furthermore, the effects of baclofen on resting potential, input resistance, firing and APs, and baclofen-induced inward rectifier potassium currents were reversibly blocked by the selective $\mathrm{GABA}_{\mathrm{B}}$ receptor antagonist CGP35348. The results verify that baclofen activates postsynaptic $G A B A_{B}$ receptors in ICD neurons.

In contrast with the findings in ICC where the IPSP(C)s were completely blocked by the selective $\mathrm{GABA}_{\mathrm{A}}$ receptor antagonist, bicuculline (Ma et al., 2002; Wu et al., 2004), stimulation of afferent fibers in the CoIC could elicited a slow and long lasting postsynaptic $\mathrm{GABA}_{\mathrm{B}}$ receptor-mediated IPSC that was completely blocked by CGP35348, indicating that $\mathrm{GABA}_{\mathrm{B}}$ receptors can mediate synaptic inhibition in the ICD. The $\mathrm{GABA}_{\mathrm{B}}$ receptor mediated synaptic responses have been observed in many other areas of the brain, e.g., hippocampus (Misgeld et al., 1995; Mott et al., 1999), globus 
pallidus (Kaneda1 and Kita, 2005), medial septum diagonal band complex (Henderson and Jones, 2005). However, despite the fact that many ICD neurons were sensitive to baclofen, the slow IPSCs were evoked by repetitive stimulation of the CoIC in about $60 \%$ (5/8) neurons that were sensitive to baclofen. The 'failure' of activation of functional $\mathrm{GABA}_{\mathrm{B}}$ receptor may be related to its extrasynaptic location and the particular anatomical features of synapses arising from CoIC afferents. If GABA released by CoIC stimulation was not sufficient to reach and activate postsynaptic $\mathrm{GABA}_{B}$ receptors, the receptors would remain silent. The results that the slow IPSCs were never elicited by single pulse of stimulation, but evoked successfully by 10 pulses of stimulation in ICD neurons support this idea. The difficulty in evoking postsynaptic $\mathrm{GABA}_{\mathrm{B}}$ receptormediated synaptic responses has been reported by other investigators (e.g., Overstreet and Westbrook, 2001; Jensen et al., 2003; Kolaj et al., 2004). Taken together, these results suggest that membrane hyperpolarization induced by baclofen and slow postsynaptic responses evoked by stimulation of the afferent fibers are attributable to activation of postsynaptic $\mathrm{GABA}_{\mathrm{B}}$ receptors through inward rectifying potassium channels.

\subsection{2. $G A B A_{B}$ receptors suppress the membrane excitability of ICD neurons}

Previous in vivo studies have shown that a reduction of sound-evoked responses of IC neurons by application of the GABAB receptor agonist, baclofen (Faingold et al., 1991; Szczepaniak and Moller, 1996; Vaughn et al., 1996). However, these electrophysiological studies can't provide evidence of the action site of baclofen. In vitro studies have shown that baclofen had an effect on GABAergic inhibition and glutamatergic excitation through a presynaptic mechanism in the ICC (Ma et al., 2002; 
Sun et al., 2006). So far there is no information about how postsynaptic $\mathrm{GABA}_{\mathrm{B}}$ receptors play a role in modulation of cell's membrane excitability and mediation of synaptic transmission in the IC. The present data demonstrated that activation of postsynaptic GABAB receptors depressed the membrane excitability of ICD neurons in several ways (Figs. 4.4 and 4.5): (1) The amplitude of the AP was decreased and the duration of the AP was increased. (2) The amplitude and duration of the AHP were reduced. (3) The firing threshold for the AP was increased. (4) The firing frequency during depolarization was decreased. The results from the voltage-clamp experiments further indicate that the enhanced membrane conductance is attributable to increasing in currents of voltage-dependent potassium channels including transient A-type of potassium channels and sustained potassium channels, and decreasing in currents of highthreshold voltage dependent calcium channels. Although hyperpolarization of membrane potential induced by baclofen may be sufficient to suppress the firing, the firing frequency was still decreased by baclofen after the resting potential was adjusted to the control level (Tennigkeit et al., 1996, 1998; Fig. 4.4). These results suggest that the suppression in membrane excitability by baclofen is a result of changes in activities of specific ion channels other than general effects of membrane hyperpolarization.

Increase of $\mathrm{K}^{+}$conductance and suppression of $\mathrm{Ca}^{2+}$ influx, the two main mechanisms, have been suggested to underlie the suppression of membrane excitability by activation of postsynaptic $G_{A B A}$ receptors in different brain areas, e.g., hippocampus (Gage, 1992; Premkumar and Gage, 1994; Gahwiler and Brown, 1985), hypothalamus (Jourdain et al., 1996; Li and Stern, 2004; Kolaj et al., 2004), thalamus (Tennigkeit et al., 1996, 1998), and trigeminal root ganglion (Takeda et al., 2004). The 
results from the present study indicate that the baclofen-induced suppression of the membrane excitability may be attributable to changes in activities of several distinct ionic currents.

\subsubsection{Modulation of voltage-dependent $K^{+}$conductance}

Several lines of evidence have shown that voltage-dependent $\mathrm{K}^{+}$channels play an important role in regulation of neuronal membrane excitability, e.g., establishing the resting membrane potential, determining the threshold of action potentials, underlying the firing patterns, governing the repolarization and hyperpolarization of the cell membrane during the generation of action potentials (Ficker and Heineman, 1992; Wu and Barish, 1992; Fu et al., 1996; Takeda et al., 2004; Sivaramakrishnan and Oliver, 2001; Kanold and Manis, 1999). Two main types of voltage dependent potassium currents, transient potassium currents (A-current, $\left.I K_{A}\right)$ and dominant sustained potassium currents $\left(I K_{S}\right)$, can be indentified by pharmacological and kinetic criteria (Wu and Barish, 1992). $I K_{A}$ is featured with rapid activation and inactivation, the dependence on the holding potential and sensitivity to 4-aminopyridine (4-AP), while $I K_{S}$ shows a higher threshold of activation compared to $I_{A}$ and high sensitivity to TEA (Wu and Barish, 1992; Fu et al., 1996).

In the present study, the buildup firing pattern was found in some ICD neurons, which was characterized by a delay in the spike generation in response to depolarizing current only or to depolarizing current preceded by a hyperpolarizing current step (Fig. 4.11A and B). $I K_{A}$ was shown to underlie the delay because it was removed during application of 4-AP. Similar firing pattern has been reported in other brain areas including the ICC (Connor and Stevens, 1971; Neher, 1971; Kim et al., 1994; Kanold and 
Manis, 1999; Perruzzi et al., 2000; Sivaramakrishnan and Oliver, 2001; Russier et al., 2003). Application of baclofen increased the amplitude of $I K_{A}$ (Fig. 4.12). This enhancement may account for the prolonged delay of the spike generation in the buildup neurons (Fig. 4.11C and D). These results are consistent with the previous findings shown in the neurons of the hippocampus (Gage, 1992) and trigeminal root ganglion (Takeda et al., 2004). The sustained $\mathrm{K}^{+}$current was also enhanced by baclofen (Fig. 4.12). The increase in both transient and sustained $\mathrm{K}^{+}$currents may contribute to the alteration in the AP properties and firing behaviour of ICD neurons by baclofen. The enhanced potassium conductance prevents the membrane from reaching the level at which a spike can be generated. Thus, the threshold of the AP was increased to a more positive level. The increased potassium current also delays the recovery of the membrane potential following an AP, resulting in reduction of the firing frequency of the neuron.

Although both voltage dependent $\mathrm{Na}^{+}$currents and voltage-dependent $\mathrm{K}^{+}$currents are involved in the generation of APs in IC neurons (Sivaramakrishnan and Oliver, 2001; Ahuja and $\mathrm{Wu}, 2007)$, there was no evidence to show the effects of baclofen on voltagedependent $\mathrm{Na}^{+}$currents in ICD neurons. The change in the shape of the AP is largely due to the modulation of activity of voltage-dependent $\mathrm{K}^{+}$channels during an AP. The increased voltage-dependent $\mathrm{K}^{+}$currents counteracted the membrane potential to reach the peak of the AP and also promoted the repolarization after the rising phase of the AP. Therefore, the amplitude of the AP was decreased, which may result in a smaller AP.

\subsubsection{Modulation of $\mathrm{Ca}^{2+}$ currents}

The movement of $\mathrm{Ca}^{2+}$ plays important roles in membrane excitability of neurons (Hille, 2001; Li et al., 1998). In many neurons the influx of calcium is mediated by 
voltage-dependent calcium channels including $\mathrm{L}, \mathrm{P} / \mathrm{Q}, \mathrm{N}, \mathrm{R}$ and low threshold $\mathrm{T}$ type $\mathrm{Ca}^{2+}$ channels (Hille, 2001). In the IC, various types of voltage-gated $\mathrm{Ca}^{2+}$ channels have been identified, including both low threshold voltage-activated (LVA) and high threshold voltage-activated (HVA) $\mathrm{Ca}^{2+}$ currents (N'gouemo and Rittenhouse, 2000; N'gouemo and Morad, 2003). In the present study, a $\mathrm{Ca}^{2+}$-mediated hump was elicited by depolarizing current injection in some ICD neurons under current clamp mode. As explained in Chapter 3, the generation of the depolarizing hump may be mediated by high threshold $\mathrm{Ca}^{2+}$ channels based on the results that the hump was elicited by about 20 $\mathrm{mV}$ positive to the resting membrane potential (McCormick, 1990; Li et al., 1998). Similar humps have been reported by previous studies in the IC (Smith, 1992; Li et al., 1998; Ahuja and Wu, 2007) and other neuronal structures (Disterhoft et al., 1993). The data in the present study showed that the hump was depressed by activation of $\mathrm{GABA}_{\mathrm{B}}$ receptors. This depression is unlikely caused by the increment in the membrane conductance because no obvious hump was observed even after the membrane potential was adjusted to the original level by a DC current (Fig. 4.10A). With voltage clamp recording, inward voltage dependent $\mathrm{Ca}^{2+}$ currents were isolated by adding TTX, TEA and 4-AP to the perfusing saline (Fig. 4.10B). The inward current was activated by large depolarizing steps and peaked at $-10 \mathrm{mV}$. The voltage dependence of the inward current in ICD neurons is similar to the high-threshold $\mathrm{Ca}^{2+}$ current reported in other neurons (Disterhoft et al 1993; Li et al., 1998). This $\mathrm{Ca}^{2+}$ current contributed to spikes in ICD neurons (Fig. 3.3). In agreement with previous investigations (Matsushima et al., 1993; Harayama et al., 1998; Bettler et al., 2004; Kolaj et al., 2004), activation of postsynaptic $\mathrm{GABA}_{\mathrm{B}}$ receptors by bath application of baclofen led to a depression of the high- 
threshold $\mathrm{Ca}^{2+}$ currents in ICD neurons. This suppression may account for the reduction of the amplitude of the AP and is believed to be mediated through G proteins by a membrane-delimited interaction between $\mathrm{Ca}^{2+}$ channels and $\mathrm{G}$ proteins (Pollo et al., 1992; Harayama et al., 1998; Bettler et al., 2004). It has been shown that different subtypes of high-threshold $\mathrm{Ca}^{2+}$ currents are depressed by activation of $\mathrm{GABA}_{\mathrm{B}}$ receptors in different neurons, e.g., L type in cerebellar granule neurons (Wojcik et al., 1990); L, N, and P/Q types in hippocampal neurons (Lambert and Wilson, 1996); $\mathrm{N}$ and $\mathrm{P} / \mathrm{Q}$ types in supraoptic neurons (Harayama et al., 1998); and $\mathrm{N}$ type in median preoptic neurons (Kolaj et al., 2004). Which subtypes of $\mathrm{Ca}^{2+}$ channels are involved in the inhibition mediated by postsynaptic $\mathrm{GABA}_{\mathrm{B}}$ receptors in the ICD requires further investigation.

\subsubsection{Modulation of $\mathrm{Ca}^{2+}$-activated potassium channels}

$\mathrm{Ca}^{2+}$ influx through voltage-dependent $\mathrm{Ca}^{2+}$ channels during the AP has several important physiological functions. For example, $\mathrm{Ca}^{2+}$ influx can activate $\mathrm{Ca}^{2+}$-dependent $\mathrm{K}^{+}$currents that underlie the AHP following a single AP and the spike broadening during repetitive firing activity with large calcium influx (Andrew and Dudek, 1985; Bevan and Wilson, 1999; Meech, 1978; Callewaert et al., 1996; Li and Stern, 2004). In the present study, most of the APs were followed by an AHP which can be partially blocked by apamin and completely eliminated by removing extracellular $\mathrm{Ca}^{2+}$. Thus, the AHP in ICD neurons may be mediated by $\mathrm{Ca}^{2+}$-dependent $\mathrm{K}^{+}$currents including $\mathrm{SK}_{\mathrm{Ca}}$ (Sivaramakrishnan and Oliver, 2001; Li and Bennett, 2007). The AHP was reduced by baclofen in ICD neurons. This reduction may be a consequence of a lower concentration of $\mathrm{Ca}^{2+}$ due to suppression of the high-threshold $\mathrm{Ca}^{2+}$ current during the AP. Similar suppressive effects on AHP by baclofen have been reported in other brain areas, e.g., 
lamprey spinal cord (Matsushima et al., 1993), supraoptic and paraventricular nuclei (Li and Stern, 2004), and median preoptic neurons (Kolaj et al., 2004). The reduction of AHP has been shown to reduce the spike-frequency adaptation and increase membrane excitability in median preoptic neurons (Kolaj et al., 2004). However, activation of $\mathrm{GABA}_{\mathrm{B}}$ receptors basically suppresses the membrane excitability including decreasing the firing frequency in ICD neurons (the present study) and in many other neurons (Tennigkeit et al., 1998; Takeda et al., 2004; Li and Stern, 2004). Therefore, although activation of $\mathrm{GABA}_{\mathrm{B}}$ receptors affects a variety of ionic conductance, some of which have opposing actions on the cell's membrane excitability, the overall outcome of activation of $\mathrm{GABA} \mathrm{B}_{\mathrm{B}}$ receptors is the suppression of the cell's excitability.

\subsection{3. $G A B A_{B}$ receptors modulate $I_{h}$ currents in ICD neurons}

The majority of the ICD neurons sampled in the present study are featured with a slowly depolarizing sag towards to the resting membrane potential during hyperpolarization, which represented the hyperpolarization-activated cation current $\left(I_{h}\right)$. $I_{h}$ has been demonstrated to be carried through mixed-cation channels that are permeable to both $\mathrm{Na}^{+}$and $\mathrm{K}^{+}$. Previous studies have compelled evidence showing that $I_{h}$ performs a wide range of physiological functions in neurons. For example, $I_{h}$ contributes to synaptic integration (Williams and Stuart, 2000), stablizing the resting membrane potential (Bal and Oertel, 2000; Doan and Kunze, 1999) and controlling the spontaneous and evoked firing (Maccaferri and McBain, 1996). In the IC, Koch and Grothe (2003) reported that $I_{h}$ also contributes to the temporal analysis of synaptic inputs. By lowering the input resistance and reducing temporal summation, $I_{h}$ improves the temporal precision in auditory processing (Oertel, 1999; Koch and Grothe, 2003). 
As expected, and in agreement with the previous studies (Jiang et al., 1993; Watts et al., 1996; Takigawa and Alzheimer, 2003), the present data show that the amplitude of $I_{h}$ was substantially reduced by activation of postsynaptic $\mathrm{GABA}_{\mathrm{B}}$ receptors during bathapplication of baclofen. This suppression was not due to the smaller membrane input resistance, because the similar depression was induced when the membrane hyperpolarization was adjusted to the same level as that in ACSF. It has been suggested that baclofen seemed to depress $I_{h}$ directly in ventral tegmental neurons. Jiang et al. (1993) reported that the suppression of $I_{h}$ by activation of $\mathrm{GABA}_{\mathrm{B}}$ receptors was mediated by activation of a $\mathrm{Gi} / \mathrm{Go}$ protein coupled intracellular pathway other than activation of inward rectifying current pathway. However, different results have been reported by several recent studies in many brain areas, e.g., substantia nigra (Watts et al., 1996), CA1 hippocampus (Takigawa and Alzheimer, 2003; Schweitzer et al., 2004). They found that the supprerssion of $I_{h}$ by activation of GABAB receptors was blocked by co-application of barium with baclofen. As barium is the blocker of inward rectifying potassium channels (Kubo et al., 1993). It should not affect activity of $\mathrm{GABA}_{\mathrm{B}}$ receptors. Therefore, it is reasonable to conclude that baclofen-induced suppression of $I_{h}$ is secondary to increasing of the inward rectifying potassium current (Watts et al., 1996; Schweitzer et al., 2004).

\subsection{4. $G_{A B A}$ receptors modulate T-type calcium channel-mediated rebound depolarization in ICD neurons}

In agreement with previous studies (Smith, 1992; Sivaramkrishnan and Oliver, 2001; Ahuja and $\mathrm{Wu}, 2007$ ), more than half of ICD neurons tested in the present study 
showed a broad rebound depolarization upon release of hyperpolarizing current pulse (Fig. 4.9A). This rebound depolarization was mediated by T-type calcium channels as explained in Chapter 3. The present results suggest that baclofen, acting on postsynaptic $\mathrm{GABA}_{\mathrm{B}}$ receptors, inhibited a T-type calcium channel-mediated rebound depolarization (Fig. 4.9). The evidence could be summarized as follows: 1) suppression of tail currents following a hyperpolarizing step (Fig. 4.9D); 2) blockade of generation of the rebound depolarization. Similar modulatory effects on T-type calcium currents by baclofen were also observed in lamprey neurons (Matsushima et al., 1993). This suppression is not a result of the decrease in membrane input resistance because larger hyperpolarization that induced a larger rebound depolarization still failed to elicit a rebound depolarization during application of baclofen. Thus, activation of $\mathrm{GABA}_{\mathrm{B}}$ receptor seems to act directly on T-type calcium channels. However, it is also possible that the baclofen-induced reduction of T-type calcium current is partially attributable to the decrease of $I_{h}$. $I_{h}$ has been demonstrated to contribute to generation of the rebound in neurons of many brain areas (Aizenmand and Linden, 1999; Sekirnjak and du Lac, 2002; Funahashi et al., 2003; Koch and Grothe, 2003; Surges et al., 2006; Russo et al., 2007). As shown in Chapter 2, $I_{h}$ also promoted generation of the rebound and associated spiking after termination of hyperpolarization in ICD neurons. My results also show that baclofen significantly suppressed $I_{h}$ in ICD neurons (Fig. 4.8). Therefore, it is reasonable to hypothesize that inhibition of the rebound by activation of $\mathrm{GABA}_{\mathrm{B}}$ receptors may be at least partially attributable to decreasing of $I_{h}$. Detailed study is required to investigate how activation of GABAB receptors affects T-type $\mathrm{Ca}^{2+}$ currents and $I_{h}$ in ICD neurons. 
The results of the present study reveal that activation of $\mathrm{GABA}_{\mathrm{B}}$ receptors can have dual functions on the rebound depolarization. First, the $\mathrm{GABA}_{\mathrm{B}}$ receptor mediated long lasting inhibitory postsynaptic response can promote generation of the rebound after the response (Ulrich and Huguenard, 1996) because the duration and amplitude of the $\mathrm{GABA}_{\mathrm{B}}$ receptor mediated response are in the range of the requirement for generation of the rebound in ICD neurons (Chapter 3 in this study). On the other hand, if $\mathrm{GABA}_{\mathrm{B}}$ receptors are activated during the period of the rebound, the rebound will be suppressed (Matsushima et al., 1993; Figs. 4.7 and 4.9). Thus, which function that the postsynaptic $\mathrm{GABA}_{\mathrm{B}}$ receptors may exert on the rebound in ICD neurons depends on timing of activation of the receptors.

\subsubsection{Synaptic transmission between ICC and ICD}

Anatomical studies have demonstrated intrinsic intracolliculus connections from the ICC to the ICD (Saldãna and Merchán, 1992, 2005). However, physiological evidence has been scarce. In most of the previous electrophysiological studies the synaptic properties of ICD neurons have been examined by stimulating the fibers of the CoIC and the lemniscus (Smith, 1992; Li et al., 1999). The results of the present study provide the first physiological evidence of intrinsic inputs from the ipsilateral ICC to the ICD. Stimulation of the ICC evoked robust postsynaptic responses. Without pharmacological manipulation, the postsynaptic responses were excitatory $(41.38 \%)$, inhibitory $(27.59 \%)$, or excitatory-inhibitory mixed $(31.03 \%)$ in different ICD neurons. Therefore, the excitatory inputs constitute the predominate component of the intrinsic connections from ICC to ICD. With the synaptic receptor antagonists, the results 
presented here clearly demonstrate that both glutamate receptors, including AMPA and NMDA receptors, and $\mathrm{GABA}_{\mathrm{A}}$ receptors mediate the synaptic transmission from ICC to ICD (Fig. 4.14C). These receptors have also been shown to mediate the synaptic transmission in the ICC (Ma et al., 2002; Wu et al., 2004; Kelly and Caspary, 2005). Further studies are required to investigate synaptic characteristics of the receptor subunits that may be related to specific functions of each subdivision in the IC.

\subsubsection{Presynaptic $\mathrm{GABA}_{B}$ receptor modulates synaptic transmission in the ICD}

In agreement with the previous studies in other brain areas, i.e., hippocampus (Lei and McBain, 2003), median preoptic nucleus (Kolaj et al., 2004), and ICC (Ma et al., 2002; Sun et al., 2006), presynaptic $\mathrm{GABA}_{\mathrm{B}}$ receptors also contribute to synaptic transmission in the ICD. For excitatory synaptic transmission, after application of baclofen both AMPA and NMDA receptor mediated EPSCs were suppressed (Fig. 4.14D and E). Baclofen application also reduced the inhibitory synaptic transmission, i.e. $\mathrm{GABA}_{\mathrm{A}}$ mediated IPSCs, in ICD neurons (Fig. 4.15). In the present study, an internal solution containing $\mathrm{Cs}^{+}$, TEA and QX314 was used to block $\mathrm{K}^{+}$currents that might be elicited in the neurons which the recording was made from. In this condition, baclofen could not activate postsynaptic $\mathrm{GABA}_{\mathrm{B}}$ receptors and therefore the modulation of glutamatergic and GABAergic synaptic transmission should be a result of action of presynaptic $\mathrm{GABA}_{B}$ receptors (Sun et al., 2006). In addition, the results from the

responses by paired-pulse stimulation also support the idea that glutamatergic and GABAergic synaptic transmission in the ICD can be regulated by $\mathrm{GABA}_{\mathrm{B}}$ receptors through a presynaptic mechanism. Similar to modulation of excitatory and inhibitory 
synaptic transmission by presynaptic $G_{A B A}$ receptors in other areas of the brain (Takahashi et al., 1998; Ma et al., 2002; Lei and McBain, 2003; Porter et al., 2004; Sun et al., 2006), presynaptic $\mathrm{GABA}_{\mathrm{B}}$ receptors may regulate both GABA and glutamate release in ICD neurons. Presynaptic $\mathrm{GABA}_{\mathrm{B}}$ receptor-induced inhibition of transmitter release has been shown to be mediated through voltage-dependent high-threshold $\mathrm{Ca}^{2+}$ channels, e.g., $\mathrm{N}$-type and $\mathrm{P} / \mathrm{Q}$ type (Bettler et al., 2004). $\mathrm{Ca}^{2+}$ influx through these channels is correlated with transmitter release by a third to fourth power law (Wu and Saggau, 1997). Thus, less than $50 \%$ depression of the $\mathrm{Ca}^{2+}$ channel activity may cause more than $90 \%$ reduction of the transmitter release (Bettler et al., 2004).

\subsubsection{Functional significance of $\mathrm{GABA}_{B}$ receptors in the ICD}

The functions of $\mathrm{GABA}_{B}$ receptors in the ICD are far from fully understood. The ICD receives GABAergic inputs mainly from the ipsilateral auditory cortex layer $\mathrm{V}$ (Winer, 2005) and the contralateral IC (Gonzalez-Hernandez et al., 1996; Saldãna and Merchán, 2005). The ICD also receives inhibitory inputs from the ipsilateral VNLL, the contralateral AVCN, PVCN, bilateral LSO and ipsilateral ICC (Gonzalez-Hernandez et al., 1996). In contrast to the ICC, strong expression of $\mathrm{GABA}_{B}$ receptors has been demonstrated in the ICD (Milbrandt et al., 1994; Fubara et al., 1996). Therefore, it seems reasonable to propose that through pre- and postsynaptic $\mathrm{GABAB}$ receptors, GABAergic inputs might dynamically modulate the neural activities of ICD neurons.

The results presented here imply that ICD neurons express postsynaptic $\mathrm{GABA}_{B}$ receptors, which is a distinct feature from that of ICC neurons. In the ICC, although the presence of $\mathrm{GABA}_{\mathrm{B}}$ receptors has been demonstrated by autoradiographic studies 
(Bowery et al., 1987; Chu et al., 1990; Milbrandt et al., 1994; Fubara et al., 1996), in vitro brain slice studies have shown little change in the membrane input resistance and firing behaviour by baclofen, suggesting the lack of postsynaptic $G_{A B} A_{B}$ receptors in ICC neurons (Ma et al., 2002). However, about $70 \%$ of the ICD neurons were sensitive to baclofen. The membrane excitability was depressed by activation of postsynaptic $\mathrm{GABA}_{\mathrm{B}}$ receptors through modulating several distinct ion channels. Postsynaptic GABA receptor may be activated concurrently with $\mathrm{GABA}_{\mathrm{A}}$ receptors, therefore, increasing the strength of synaptic inhibition. In addition, activation of postsynaptic $\mathrm{GABA}_{\mathrm{B}}$ receptors prolongs the return of the membrane potential to the resting level, allowing deinactivation of T-type calcium channels and generation of a rebound depolarization (Ulrich and Huguenard, 1996). All these actions of postsynaptic GABA $A_{B}$ receptors provide mechanisms of modulation of neural activities of ICD neurons.

Presynaptic $\mathrm{GABA}_{\mathrm{B}}$ receptor plays a role in regulation of synaptic strength in ICD neurons. Depending on whether activation of presynaptic $\mathrm{GABA}_{\mathrm{B}}$ receptors occurs on glutamatergic or GABAergic terminals, the effects on the postsynaptic neuron can be inhibitory or disinhibitory, respectively. As the responses of an ICD neuron to acoustic stimulation is a reflection of a complex integration of excitation and inhibition, presynaptic $\mathrm{GABA}_{\mathrm{B}}$ receptor can be involved in regulating the balance of excitatory and inhibitory inputs during an auditory stimulus, thus, preventing overstimulation in ICD neurons.

Taken together, the pre- and postsynaptic $\mathrm{GABA}_{\mathrm{B}}$ receptors in ICD neurons may play significant roles in auditory signal processing. 


\section{Chapter 5 Summary}

To understand better the physiological role of the ICD in auditory processing, in the present dissertation I examined the physiological characteristics of ICD neurons using in vitro whole-cell patch clamp techniques. Three studies were performed.

The first study specifically investigated intrinsic membrane properties of ICD neurons and modulation of membrane excitability by preceding hyperpolarization. According to firing patterns in response to depolarizing current injection, different cell types were classified: three major types (sustained-regular, sustained-adapting, buildup) and others including fast adapting, transient, and onset. The intrinsic membrane properties of ICD neurons are similar across the cell types and animal ages. More than half $(51.7 \%)$ of the sustained neurons showed rebound depolarization upon the release of the preceding hyperpolarization. Rebound depolarization and spikes after hyperpolarization were not seen in the buildup neurons. When depolarizing current was preceded by a hyperpolarizing current, various forms of the modification on membrane excitability were observed. For non-rebound neurons, the membrane excitability was either suppressed or unchanged after pre-hyperpolarization. The first spike latency lengthened in neurons whose firing changed to a buildup pattern, shortened in those whose firing changed to a pauser pattern, and remained unchanged in those whose discharge pattern remained sustained. For rebound neurons, the firing rate decreased in neurons whose firing pattern was changed to onset or pauser, increased in neurons whose firing was changed to adapting, or remained unchanged in neurons whose firing became irregular. The first spike latency was shortened in all the rebound cells. The results suggest that intrinsic membrane properties can play an important role in integration of 
excitatory and inhibitory inputs and thereby in determination of the output of ICD neurons.

The second study focused on the contribution of calcium to the membrane excitability of ICD neurons. Slow afterhyperpolarization following an action potential was observed in the majority of ICD neurons and it was partially attributable to activation of $\mathrm{Ca}^{2+}$-activated $\mathrm{K}^{+}$channels with small conductance $\left(\mathrm{SK}_{\mathrm{Ca}}\right) . \mathrm{Ca}^{2+}$ also mediated the hump and regenerative spikes induced by membrane depolarization. In more than half of the ICD neurons, there was a rebound depolarization accompanied by anode break action potentials following membrane hyperpolarization. The rebound was $\mathrm{Ca}^{2+}$ mediated and was primarily due to activation of low-threshold T-type $\mathrm{Ca}^{2+}$ channels. Generation of the rebound and anode break spikes depended on magnitude and duration of preceding hyperpolarization when the level of hyperpolarization was small. However, once the hyperpolarization was sufficiently large, the rebound became constant in amplitude and duration as the strength or duration of the hyperpolarization was increased. All the results suggest that activation of $\mathrm{Ca}^{2+}$ current and $\mathrm{Ca}^{2+}$-dependent $\mathrm{K}^{+}$current regulates membrane excitability not only when the neuron was depolarized, but also when it returns to the resting state following hyperpolarization. These $\mathrm{Ca}^{2+}$ mediated events may play important roles in processing specific aspects of auditory information.

The last study examined the physiological role of pre- and postsynaptic $\mathrm{GABA}_{\mathrm{B}}$ receptors in ICD neurons. Postsynaptic effects of $\mathrm{GABA}_{\mathrm{B}}$ receptors were manifested in about $70 \%$ of the ICD neurons. In current clamp mode, $\mathrm{GABA}_{\mathrm{B}}$ receptor agonist, baclofen, reversibly hyperpolarized the cell membrane and the hyperpolarization was associated with a decrease in the neuron's input resistance. Baclofen depressed the firing 
activity of ICD neurons, which was accompanied by a reduction in the individual AP and AHP amplitude and the duration of the AHP, and an increase in the duration of the individual AP. Under voltage clamp, baclofen induced an inward rectifying potassium current that can be abolished by the $\mathrm{K}^{+}$channel blocker barium. Application of the specific $\mathrm{GABA}_{B}$ receptor antagonist, CGP35348, reversed the effects of baclofen. Stimulation of afferent fibers in the CoIC could elicit a slow and long lasting postsynaptic $\mathrm{GABA}_{\mathrm{B}}$ receptor-mediated IPSC that was completely blocked by CGP35348 in ICD neurons. This study provides direct evidence for the presence of postsynaptic $\mathrm{GABA}_{\mathrm{B}}$ receptors that are coupled to inward rectifying $\mathrm{K}^{+}$channels in ICD neurons. Activation of postsynaptic $\mathrm{GABA}_{\mathrm{B}}$ receptors modulated several distinct ionic conductances, i.e., depressing $I_{h}$, low-threshold T type calcium current, high-threshold calcium current, and calcium activated potassium current, and increasing the sustained and transient A-type $\mathrm{K}^{+}$ currents. The results also demonstrated that both glutamate receptors, including AMPA and NMDA receptors, and $\mathrm{GABA}_{\mathrm{A}}$ receptors mediate the synaptic transmission from ICC to ICD. Baclofen application decreased the amplitude of evoked AMPA and NMDA EPSCs, and GABA $A_{A}$ IPSCs. The effects of baclofen were blocked by CGP35348. Baclofen also enhanced the paired-pulse ratio of evoked IPSCs. Therefore, these results support the expression of both pre- and postsynaptic $\mathrm{GABA}_{\mathrm{B}}$ receptors in ICD neurons. Activation of postsynaptic $\mathrm{GABA}_{\mathrm{B}}$ receptors efficiently modulates membrane excitability through different ionic currents, while presynaptic $\mathrm{GABA}_{B}$ receptors contribute to the modulation of both glutamatergic and GABAergic transmission in the ICD.

In summary, the data from the present study and the limited earlier findings indicate that the ICD may be involved in processing auditory information through a way 
that is different from the ICC. Although ICC and ICD neurons share similar membrane properties including distinct firing patterns in response to depolarizing current injection and rebound depolarization following hyperpolarizing current pulses (Sivaramkrishnan and Oliver, 2001; Sun and Wu, 2007), the ICD shows many unique synaptic features. In contrast to the ICC, the ICD receives only limited direct projections from the lower auditory brainstem. Most auditory inputs to the ICD are from the contralateral IC, ipsilateral ICC and ICX, and auditory cortex (Schofield, 2005). In the ICD, both pre- and postsynaptic $\mathrm{GABA}_{\mathrm{B}}$ receptors are involved in $\mathrm{GABA}_{\mathrm{B}}$-induced inhibition (the present study). In addition, compared to ICC neurons, ICD neurons are poorly responsive to acoustic stimuli, tend to habituate, and have broader tuning curves (Syka et al., 2000). Therefore, these response properties may correspond with the role of the ICD in modulating and refining the processing of the auditory information from the lower auditory nuclei to the auditory cortex. 


\section{References}

Adams JC (1980) Crossed and descending projections to the inferior colliculus. Neurosci Lett 19:1-5.

Adams JC, Mugnaini E (1984) Dorsal nucleus of the lateral lemniscus: A nucleus of GABAergic projection neurons. Brain Res Bull 13: 585-590.

Ahuja TK, Wu SH (2000) Developmental changes in physiological properties in the rat's dorsal nucleus of the lateral lemniscus. Hear Res 3531: 1-13.

Ahuja TK, Wu SH (2007) Intrinsic membrane properties and synaptic response characteristics of neurons in the rat's external cortex of the inferior colliculus. Neurosci 145:851-865.

Aitkin L, Tran L, Syka J (1994) The responses of neurons in subdivisions of the inferior colliculus of cats to tonal, noise and vocal stimuli. Exp Brain Res 98: 53-64.

Aitkin LM, Dickhaus H, Schult W, Zimmermann M (1978) External Nucleus of inferior colliculus: auditory and spinal somatosensory afferents and their interactions. $J$ Neurophysiol 41(4): 837-847.

Aitkin LM, Kenyon CE, Philpott P (1981) The representation of the auditory and somatosensory systems in the external nucleus of the cat inferior colliculus. $J$ Comp Neurol 196: 25-40.

Aitkin LM, Phillips SC (1984) The interconnections of the inferior colliculus through their commissure. J Comp Neurol 228: 210-216.

Aitkin LM, Webster WR, Veale JL, Crosby DC (1975) Inferior colliculus. I. Comparison of response properties of neurons in the central, pericentral, and external nuclei of the adult cat. J Neurophysiol 41: 837-847.

Aizenman CD, Linden DJ (1999) Regulation of the rebound depolarization and spontaneous firing patterns of deep nuclear neurons in slices of rat cerebellum. $J$ Neurophysiol 82(4):1697-709.

Alexander GM, Carden WB, Mu J, Kurukulasuriya NC, McCool BA, Nordskog BK, Friedman DP, Daunais JB, Grant KA, Godmin DW (2006) The native T-type calcium current in relay neurons of the primate thalamus. Neurosci 141: 453-461.

Andersen RA, Roth GL, Aitkin LM, Merzenich MM (1980) The efferent projections of the central nucleus and the pericentral nucleus of the inferior colliculus in the cat. J Comp Neurol 194:649-62.

Andrew RD, Dudek FE (1985) Spike broadening in magnocellular neuroendocrine cells of rat hypothalamic slices. Brain Res 334(1):176-9.

Angelotti TP and Macdonald RL (1993) Assembly of GABA A $_{A}$ receptor subunits: Alpha 1 beta 1 and alpha 1 beta 1 gamma $2 S$ subunits produce unique ion 
channels with dissimilar single-channel properties. $J$ Neurosci 13: 1429-1440.

Arnault P, Roger M (1990) Ventral temporal cortex in the rat: connections of secondary auditory areas Te 2 and Te 3. J Comp Neurol 302: 110-123.

Bajo VM, Moore DR (2006) Descending projections from the auditory cortex to the inferior colliculus in the gerbil, Meriones unguiculatus. $J$ Comp Neurol 486:101-116.

Bal R, Oertel D (2000) Hyperpolarization-activated, mixed-cation current (I(h)) in octopus cells of the mammalian cochlear nucleus. J Neurophysiol 84(2):806-17.

Banks MI, Pearce RA, Smith PH (1993) Hyperpolarization-activated cation current (Ih) in neurons of the medial nucleus of the trapezoid body: voltage-clamp analysis and enhancement of norepinephrine and cAMP suggest a modulatory mechanism in the auditory brain stem. $J$ Neurophysiol 70: 1420-1432.

Barnard EA, Skolnick P, Olsen RW, Mohler H, Sieghart G, Biggio G, Braestrup C, Bateson AN and Langer SZ (1998) International Union Of Pharmacology. XV. Subtypes of G-aminobutyric acidA receptors: Classification on the basis of subunit structure and receptor function. Pharmacol Rev 50: 291-313.

Bayliss DA, Viana F, Bellingham MC, Berger AJ (1994) Characteristics and postnatal development of a hyperpolarization-activated inward current in rat hypglossal motorneurons in vitro. J Neurophysiol 71: 119-128.

Behe P, Stern P, Wyllie DJ, Nassar M, Schoepfer R, Colquhoun D (1995) Determination of NMDA NR1 subunit copy number in recombinant NMDA receptors. Proc R Soc Lond B Biol Sci 262: 205-213.

Benke D, Honer M, Michel C, Bettler B, Mohler H (1999) Gamma-aminobutyric acid type B receptor splice variant proteins GBR1a and GBR1b are both associated with GBR2 in situ and display differential regional and subcellular distribution. $J$ Biol Chem 274: 27323-27330.

Berthele A, Platzer S, Weis S, Conrad B, Tölle TR (2001) Expression of GABA(B1) and GABA(B2) mRNA in the human brain. Neuroreport 12(15):3269-75.

Bettler B, Kaupmann K, Mosbacher J, Gassmann M (2004) Molecular structure and physiological functions of GABA(B) receptors. Physiol Rev 84(3):835-67.

Beurrier C, Congar P, Bioulac B, Hammond C (1999) Subthalamic nucleus neurons switch from single-spike activity to burst-firing mode. J Neurosci 19: 599-609.

Bevan MD, Magill PJ, Hallworth NE, Bolam JP, Wilson CJ (2002) Regulation of the timing and pattern of action potential generation in rat subthalamic neurons in vitro by GABA-A IPSPs. $J$ Neurophsiol 87, 1348-1362.

Bevan MD, Wilson CJ (1999) Mechanisms underlying spontaneous oscillation and rhythmic firing in rat subthalamic neurons. J Neurosci 19(17):7617-7628. 
Beyerl BD (1978) Afferent projections to the central nucleus of the inferior colliculus in the rat. Brain Res 145: 209-223.

Billinton A, Upton N, and Bowery NG (1999) $\mathrm{GABA}_{B}$ receptor isoforms GBR1a and GBR1b, appear to be associated with pre- and post-synaptic elements respectively in rat and human cerebellum. Br J Pharmacol 126: 1387-1392.

Bischoff S, Leonhard S, Reymann N, Schuler V, Shigemoto R, Kaupmann K, and Bettler B (1999) Spatial distribution of GABA(B)R1 receptor mRNA and binding sites in the rat brain. $J$ Comp Neurol 412: 1-16,

Bonanno G, Raiteri M (1993) gamma-Aminobutyric acid (GABA) autoreceptors in rat cerebral cortex and spinal cord represent pharmacologically distinct subtypes of the GABA $A_{B}$ receptor. J Pharmacol Exp Ther 265(2): 765-70.

Boulter J, Hollmann M, O'Shea-Greenfield A, Hartley M, Deneris E, Maron C, Heinemann S. (1990) Molecular cloning and functional expression of glutamate receptor subunit genes. Science 249(4972):1033-7.

Bowery NG (1993) GABA B $_{\mathrm{B}}$ receptor pharmacology. Annu Rev Pharmacol Toxicol 33: 109-147.

Bowery NG (2006) $\mathrm{GABA}_{\mathrm{B}}$ receptor: a site of therapeutic benefit. Curr Opin Pharmacol 6(1):37-43.

Bowery NG, Bettler B, Froestl W, Gallagher JP, Marshall F, Raiteri M, Bonner TI, Enna SJ (2002) International Union of Pharmacology. XXXIII. Mammalian gamma-aminobutyric acid (B) receptors: structure and function. Pharmacol Rev 54(2):247-64.

Bowery NG, Hudson AL, Price GW (1987) $\mathrm{GABA}_{\mathrm{A}}$ and $\mathrm{GABA}_{\mathrm{B}}$ receptor site distribution in the rat central nervous system. Neuroscience 20(2):365-83.

Brand A, Urban A, Grothe B (2000) Duration tuning in the mouse auditory midbrain. $J$ Neurophysiol 84:1790-1799.

Brauner-Osborne H, Krogsgaard-Larsen P (1999) Functional pharmacology of cloned heterodimeric $\mathrm{GABA}_{\mathrm{B}}$ receptors expressed in mammalian cells. $\mathrm{Br} J$ Pharmacol 128(7): 1370-4.

Bristow DR, Martin IL (1988) Light microscopic autoradiographic localisation in rat brain of the binding sites for the $\mathrm{GABA}_{\mathrm{A}}$ receptor antagonist [3H]SR 95531: comparison with the $[3 \mathrm{H}] \mathrm{GABA}_{\mathrm{A}}$ receptor distribution. Eur J Pharmacol 148(2): 283-8.

Browning RA, Lanker ML, Faingold CL (1989) Injections of noradrenergic and GABAergic agonists into the inferior colliculus: effects on audiogenic seizures in genetically epilepsy prone rats. Epilepsy Res 4: 119-125.

Browning RA, Nelson, DK, Mogharreban N, Jobe PC, Laird HE (1985) Effect of midbrain and pontine tegmental lesions on audiogenic seizures in genetically 
epilepsy-prone rats. Epilepsia 26: 175-183.

Brunso-Bechtold JK, Thompson GC, Masterton RR (1981) HRP study of the organization of auditory afferents ascending to central nucleus of inferior colliculus in cat. J Comp Neurol 197: 705-722.

Burger RM, Pollak GD (1998) Analysis of the role of inhibition in shaping responses to sinusoidally amplitude-modulated signals in the inferior colliculus. $J$ Neurophysiol 80(4):1686-701.

Byrne JH, Kandel ER (1996) Presynaptic facilitation revisited:state and time dependence. $J$ Neurosci 16: 425-435.

Caicedo A, Eybalin M (1999) Glutamate receptor phenotypes in the auditory brainstem and mid-brain of the developing rat. Eur J Neurosci 11(1):51-74.

Cajal SR (1909) Histologie du systeme nerveux de l'homme et des vertebras. $A$ Maloine (Paris): 153-173.

Calford MB, Aitkin LM (1983) Ascending projections to the medial geniculate body of the cat: evidence for multiple, parallel auditory pathways through thalamus. $J$ Neurosci 3(11):2365-80.

Callewaert G, Eilers J, Konnerth A (1996) Axonal calcium entry during fast 'sodium' action potentials in rat cerebellar Purkinje neurones. $J$ Physiol 495:641-7.

Calver AR, Davies $\mathrm{CH}$, Pangalos $\mathrm{M}$ (2002) $\mathrm{GABA}_{\mathrm{B}}$ receptors: from monogamy to promiscuity. Neurosignals 11(6):299-314.

Calver AR, Medhurst AD, Robbins MJ, Charles KJ, Evans ML, Harrison DC, Stammers M, Hughes SA, Hervieu G, Couve A, Moss SJ, Middlemiss DN, Pangalos $\mathrm{MN}(2000)$ The expression of $\mathrm{GABA}_{\mathrm{B} 1}$ and $\mathrm{GABA}_{\mathrm{B} 2}$ receptor subunits in the CNS differs from that in peripheral tissues. Neuroscience 100(1): 155-70.

Calver AR, Robbins MJ, Cosio C (2001) The C-terminal domains of the GABABreceptor subunits mediate intracellular trafficking but are not required forreceptor signaling. $J$ Neurosci 1203-1210.

Cant NB (2005) Projections from the Cochlear Nuclear Complex to the Inferior Colliculus. In: The Inferior Colliculus (Winer JA, Schreiner CE, eds) pp 115-131 New York: Springer-Verlag.

Carbone E, Lux HD (1984) A low voltage-activated, fully inactivating Ca channel in vertebrate sensory neurons. Nature 310: 501-502.

Caspary DM, Holder TM, Milbrandt JC, McKernan R, Naritoku DK (1999) Age-related changes in $\mathrm{GABA}_{\mathrm{A}}$ receptor subunit composition and function in the rat auditory system. Neuroscience 93: 307-312.

Caspary DM, Raza A, Lawhorn Armour BA, Pippin J, Arnerić SP (1990) Immunocytochemical and neurochemical evidence for age-related loss of GABA 
in the inferior colliculus: implications for neural presbycusis. $J$ Neurosci $10(7)$ : 2363-72.

Casseday JH, Ehrlich D, Covey E (1994) Neural tuning for sound duration: role of inhibitory mechanisms in the inferior colliculus. Science 264:847-850.

Casseday JH, Ehrlich D, Covey E (2000) Neural measurement of sound duration: control by excitatory-inhibitory interactions in the inferior colliculus. $J$ Neurophysiol 84:1475-1487.

Casseday JH, Fremouw T, and Covey E (2002) The inferior colliculus: hub of the auditory system. In: Integrative Functions in the Mammalian Auditory Pathway (Oertel D, Popper AN, Fay RR, eds) pp 238-318 New York: Springer-Verlag.

Casseday JH, Jones DR, Diamond IT (1979) Projections from cortex to tectum in the tree shrew. Tupaia glis. J Comp Neurol 185: 253-292.

Chakravarty DN, Faingold CL (1996) Increased responsiveness and failure of habituation in neurons of the external nucleus of inferior colliculus associated with audiogenic seizures of the genetically epilepsy-prone rat. Exp Neurol 141: 280-286.

Chatterton JE, Awobuluyi M, Premkumar LS, Takahashi H, Talantova M, Shin Y, Cui J, Tu S, Sevarino KA, Nakanishi N, Tong G, Lipton SA, Zhang D (2002) Excitatory glycine receptors containing the NR3 family of NMDA receptor subunits. Nature 415(6873):793-8.

Chen C (1997) Hyperpolarization-activated current (Ih) in primary auditory neurons. Hear Res 110: 179-190.

Chu DC, Albin RL, Young AB, Penney JB (1990) Distribution and kinetics of GABAB binding sites in rat central nervous system: a quantitative autoradiographic study. Neuroscience 34(2):341-57.

Clark JA, Mezey E, Lam AS, Bonner TI (2000) Distribution of the GABA(B) receptor subunit gb2 in rat CNS. Brain Research 860: 41-52.

Coleman J, Rainer R, Clerici WJ (1984) Projection patterns of rat auditory cortex. Soc Neurosci Abstr 12: 247.

Coleman JR, Clerici WJ (1987) Sources of projections to subdivisions of the inferior colliculus in the rat. J Comp Neurol 262: 215-226.

Connor JA, Stevens CF (1971) Prediction of repetitive firing behavior from voltage clamp data on an isolated neurone soma. $J$ Physiol (Lond) 213:31-53.

Connor JA, Stevens CF (1971) Voltage clamp studies of a transient outward membrane current in gastropod neural somata. $J$ Physiol (Lond) 213: 21-30.

Covey E, Casseday JH (1999) Timing in the auditory system of the bat. Annu Rev Physiol 61:457-476. 
Covey E, Hall WC, Kobler JB (1987) Subcortical connections of the superior colliculus in the mustache bat, Pteronotus parellii. J Comp Neurol 263: 179-197.

Covey E, Kauer JA, Casseday JH (1996) Whole-cell patch-clamp recording reveals subthreshold sound-evoked postsynaptic currents in the inferior colliculus of awake bats. $J$ Neurosci 16:3009-3018.

Cruneli V, Leresche $\mathrm{N}$ (1991) A role for $\mathrm{GABA}_{\mathrm{B}}$ receptors in excitation and inhibition of thalamocortical cells. Trends Neurosci 14(1):16-21.

D'Angelo WR, Sterbing SJ, Ostapoff EM, Kuwada S (2005) Role of GABAergic inhibition in the coding of interaural time differences of low-frequency sounds in the inferior colliculus. $J$ Neurophysiol 93(6):3390-400.

Das S, Sasaki YF, Rothe T, Premkumar LS, Takasu M, Crandall JE, Dikkes P, Conner DA, Rayudu PV, Cheung W, Chen HS, Lipton SA, Nakanishi N (1998) Increased NMDA current and spine density in mice lacking the NMDA receptor subunit NR3A. Nature 393:377-381.

Davies CH, Davies SN, Collingridge GL (1990) Paired-pulse depression of monosynaptic GABA-mediated inhibitory postsynaptic responses in rat hippocampus. $J$ Physiol (Lond) 424: 513-531.

Disterhoft JF, Moyer JR Jr, Thompson LT, Kowalska M (1993) Functional aspects of calcium-channel modulation. Clin Neuropharmacol 16:S12-24.

Doan TN, Kunze DL (1999) Contribution of the hyperpolarization-activated current to the resting membrane potential of rat nodose sensory neurons. J Physiol 514 ( Pt $1): 125-38$.

Druga R, Syka J (1984) Ascending and descending projections to the inferior colliculus in the rat. Physiol Bohemoslov 33: 31-41.

Druga R, Syka J (2001) Effect of auditory cortex lesions on NADPH-diaphorase staining in the inferior colliculus of rat. Neuroreport 12: 1555-1559.

Druga R, Syka J, Rajkowska G (1997) Projections of auditory cortex onto the inferior colliculus in the rat. Physiol Res 46: 215-222.

Durkin MM, Gunwaldsen CA, Borowsky B, Jones KA, Branchek TA (1999) An in situ hybridization study of the distribution of the GABA(B2) protein mRNA in the rat CNS. Brain Res Mol Brain Res 71(2):185-200.

Ebert U, Ostwald J (1992) Serotonin modulates auditory information processing in the cochlear nucleus of the rat. Neurosci Lett 145: 51-54.

Edwards SB, Ginsburgh CL, Jenkel CK, Stein BE (1979) Sources of subcortical projections to the superior colliculus in the cat. J Comp Neurol 184: 309-330.

Edwards SB, Henkel CK (1978) superior colliculus connections with extraocular motor nuclei in the cat. J Comp Neurol 179: 451-467. 
Ehret G (1997) The auditory midbrain, a "shunting yard" of acoustical information processing. In: The central auditory system. Chpt.4. ed. Ehret, G., and Romand, R., Oxford University Press.

Ehrlich D, Casseday JH, Covey E (1997) Neural tuning to sound duration in the inferior colliculus of the big brown bat, Eptesicus fuscus. $J$ Neurophysiol $77: 2360-2372$.

Evans MS, Viola-McCabe KE, Caspary DM, Faingold CL (1994) Loss of Loss of synaptic inhibition during repetitive stimulation in genetically epilepsy-prone rats (GEPR). Epilepsy Res 18(2):97-105.

Faber ES, Sah P (2002) Physiological role of calcium-activated potassium currents in the rat lateral amygdala. $J$ Neurosci $22,1618-1628$.

Faingold CL (2002) Role of GABA abnormalities in the inferior colliculus pathophysiology-audiogenic seizures. Hear Res 168:223-237.

Faingold CL (2005) The midbrain and audiogenic seizures. In: The Inferior Colliculus (Winer JA, Schreiner CE, eds) pp 603-626 New York: Springer-Verlag.

Faingold CL, Anderson CAB, Randall, ME (1993) Stimulation or blockade of the dorsal nucleus of the lateral lemniscus alters binaural and tonic inhibition in contralateral inferior colliculus neurons. Hear Res 69: 98-106.

Faingold CL, Boersma Anderson CA, Caspary DM (1991) Involvement of GABA in acoustically-evoked inhibition in inferior colliculus neurons. Hear Res 52: 201-216.

Faingold CL, Casebeer DJ (1999) Modulation of the audiogenic seizure network by noradrenergic and glutamatergic receptors of the deep layers of superior colliculus. Brain Res 821: 392-399.

Faingold CL, Gehlbach G, Caspary DM (1989) The role of GABA as an inhibitory neurotransmitter in inferior colliculus neurons: iontophoretic studies. Brain Res 500: $302-312$.

Faingold CL, Gehlbach G, Caspary DM (1991) Functional pharmacology of inferior colliculus neurons. In: Altschuler.R.A., Bobbin, R.P., Clopton, B.M., Hoffman, D.W. (Eds.), Neurobiology of Hearing: The Central Auditory System. Raven, Press, New York, pp 223-251.

Faingold CL, Gehlbach G, Travis MA, Caspary DM (1986) Inferior colliculus neuronal response abnormalities in genetically epilepsy-prone rats and evidence for a deficit of inhibition. Life Sci 39: 869-878.

Faingold CL, N'Gouemo P, Riaz A (1998) Ethanol and neurotransmitter interactions from molecular to integrative effects. Prog Neurobiol 55: 509-535.

Faingold CL, Randall ME (1999) Neurons in the deep layers of superior colliculus play a critical role in the neuronal network for audiogenic seizures: mechanisms 
for production of wild running behaviour. Brain Res 815 : 250-258.

Fan YP, Horn EM, Waldrop TG (2000) Biophysical characterization of rat caudal hypothalamic neurons: calcium channel contribution to excitability. $J$ Neurophysiol 84:2896-2903.

Faure PA, Premouw T, Casseday JH, Covey E (2003) Temporal masking reveals properties of sound-evoked inhibition in duration-tuned neurons of the inferior colliculus. $J$ Neurosci 23:3052-3065.

Faye-Lund H (1985) The neocortical projection to the inferior colliculus in the albino rat. Anat Embryol 173:53-70.

Faye-Lund H (1986) Projection from the inferior colliculus to the superior olivary complex in the albino rat. Anat Embryol 175: 35-52.

Faye-Lund H, Osen KK (1985) Anatomy of the inferior colliculus in rat. Anat Embryol 171: 1-20.

Feldman SG, Knudsen EI (1994) NMDA and non-NMDA glutamate receptors in auditory transmission in the barn owl inferior colliculus. $J$ Neurosci 14: 5939-5958.

Feldman SG, Kruger L (1980) An axonal transport study of the ascending projection of medial lemniscal neurons in the rat. JComp Neurol 192: 427-454.

Filippov AK, Couve A, Pangalos MN, Walsh FS, Brown DA, Moss SJ (2000) Heteromeric assembly of $\mathrm{GABA}(\mathrm{B}) \mathrm{R} 1$ and $\mathrm{GABA}(\mathrm{B}) \mathrm{R} 2$ receptor subunits inhibits $\mathrm{Ca}(2+)$ current in sympathetic neurons. J Neurosci 20(8):2867-74.

Fitzgerald KK, Sanes DH (1999) Serotonergic modulation of synapses in the developing gerbil lateral superior olive. J Neurophysiol 81: 2743-2752.

Fitzpatrick KA (1975) Cellular architecture and topographic organization of the inferior colliculus of the squirrel monkey. JComp Neurol 164: 185-208.

Foster RE, Connors BW, Waxman SG (1982) Rat optic nerve: electrophysiological, pharmacological and anatomical studies during development. Brain Res 255: 371-386.

Fremouw, A. Kleiser, A. Heilman, J.H. Casseday and E. Covey (1999) Intrinsic and commissural GABAergic connections in the inferior colliculus of the big brown bat, Neurosci Abstr 25: p. 1417.

Frisina RD, O'Neill WE, Zettel ML (1989) Functional organization of mustached bat inferior colliculus: II. Connections of the FM2 region. J Comp Neurol 284: 85-107.

Frisina RD, Walton JP, Lynch-Armour MA, Klotz DA (1997) Efferent projections of a physiologically characterized region of the inferior colliculus of the young adult CBA mouse. $J$ Acoust Soc Am 101:2741-2753. 
Fritschy JM, Meskenaite V, Weinmann O, Honer M, Benke D, and Mohler H (1999) GABAB-receptors splice variants GB1a and GB1b in rat brain: developmental regulation, cellular distribution and extrasynaptic localization. Eur $J$ Neurosci 11: 761-768.

Fritschy JM, Sidler C, Parpan F, Gassmann M, Kaupmann K, Bettler B, Benke D (2004) Independent maturation of the $\mathrm{GABA}(\mathrm{B})$ receptor subunits $\mathrm{GABA}(\mathrm{B} 1)$ and GABA(B2) during postnatal development in rodent brain. J Comp Neurol 477(3): $235-52$.

Fu XW, Brezden BL, Wu SH (1997) Hyperpolarization-activated inward current in neurons of the rat's dorsal nucleus of the lateral lemniscus in vitro. $J$ Neurophysiol 78: 2235-2245.

Fu XW, Wu SH, Brezden BL, Kelly JB (1996) Potassium currents and membrane excitability of neurons in the rat's dorsal nucleus of the lateral lemniscus. $J$ Neurophysiol 76(2):1121-32.

Fubara BM, Casseday JH, Covey E, Schwartz-Bloom RD (1996) Distribution of $\mathrm{GABA}_{\mathrm{A}}, \mathrm{GABA}_{\mathrm{B}}$, and glycine receptors in the central auditory system of the big brown bat, Eptesicus fuscus. J Comp Neurol 369:83-92.

Fujita I, Konishi M (1991) The role of GABAergic inhibition in processing of interaural time difference in the owl's auditory system. J Neurosci 11(3):722-39.

Funahashi M, Mitoh Y, Kohjitani A, Matsuo R (2003) Role of the hyperpolarization-activated current (Ih) in pacemaker activity in area postrema neurons of rat brain slices. $J$ Physiol 552:135-148.

Fuzessery ZM, Hall JC (1996) Role of GABA in shaping frequency tuning and creating FM sweep selectivity in the inferior colliculus. $J$ Neurophysiol 76(2):1059-73.

Fuzessery ZM, Richardson MD, Coburn MS (2006) Neural mechanisms underlying selectivity for the rate and direction of frequency-modulated sweeps in the inferior colliculus of the pallid bat. J Neurophysiol 96:1320-1336.

Gage PW (1992) Activation and modulation of neuronal $\mathrm{K}^{+}$channels by GABA. Trends Neurosci 15(2):46-51.

Gähwiler $\mathrm{BH}$, Brown DA (1985) $\mathrm{GABA}_{\mathrm{B}}$-receptor-activated $\mathrm{K}^{+}$current in voltage-clamped CA3 pyramidal cells in hippocampal cultures. Proc Natl Acad Sci 82(5):1558-62.

Galazyuk AV, Lin W, Llano D, Feng AS (2005) Leading inhibition to neural oscillation is important for time-domain processing in the auditory midbrain. $J$ Neurophysiol 94: 314-326.

Gaza WC, Ribak CE (1997) Immunocytochemical localization of AMPA receptors in the rat inferior colliculus. Brain Res 774(1-2):175-83. 
Geniec P, Morest DK (1971) The neuronal architecture of the human posterior colliculus. Acta Otolaryngol Supp 295: 1-33.

Glendenning KK, Baker BN (1988) Neuroanatomical distribution of receptors for three potential inhibitory neurotransmitters in the brainstem auditory nuclei of the cat. J Comp Neurol 272: 288-308.

Glendenning KK, Baker BN, Hutson KA, Masterton RB (1992) Acoustic chiasm V: inhibition and excitation in the ipsilateral and contralateral projections of LSO. $J$ Comp Neurol 319: 100-122.

Golding NL, Robertson D, Oertel D (1995) Recordings from slices indicate that octopus cells of the cochlear nucleus detect coincident firing of auditory nerve fibers with temporal precision. $J$ Neurosci 15 : 3138-3153.

González-Hernández T, Mantolan B, González B, Pérez H (1996) Sources of GABAergic input to the inferior colliculus of the rat. $J$ Comp Neurol 372:309-326.

Gutnick MJ, Conners BW, Prince DA (1982) Mechanisms of neocortical epileptogenesis in vitro. $J$ Neurophysiol 48: 1321-1335.

Hallworth NE, Wislon CJ, Bevan MD (2003) Apamin-sensitive small conductance calcium-activated potassium channels, through their selective coupling to voltage-gated calcium channels, are critical determinants of the precision, pace, and pattern of action potential generation in rat subthalamic nucleus neurons in vitro. J Neurosci 23: 7525-7542.

Harayama N, Shibuya I, Tanaka K, Kabashima N, Ueta Y, Yamashita H (1998) Inhibition of $\mathrm{N}$ - and $\mathrm{P} / \mathrm{Q}$-type calcium channels by postsynaptic $\mathrm{GABA}_{\mathrm{B}}$ receptor activation in rat supraoptic neurones. $J$ Physiol 509 (Pt 2):371-83.

Hashikawa $\mathrm{T}$ (1983) The inferior colliculopontine neurons of the cat in relation to other collicular descending neurons. J Comp Neurol 219: 241-249.

Hashikawa T, Kawamura K (1983) Retrograde labeling of ascending and descending neurons in the inferior colliculus. A fluorescent double labeling study in the cat. Exp Brain Res 49: 457-461.

Hefti BJ, Smith PH (2000) Anatomy, physiology, and synaptic responses of rat layer $\mathrm{V}$ auditory cortical cells and effects of intracellular GABA(A) blockade. $J$ neurophysiol 83(5):2626-38.

Henderson Z, Jones GA (2005) GABAB receptors in the medial septum/diagonal band slice from 16-25 day rat. Neuroscience 132(3):789-800.

Henkel CK, Spangler KM (1983) Organization of the efferent projections of the medial olivary nucleus in the cat as revealed by HRP and autoradiographic tracing methods. J Comp Neurol 221: 416-428.

Herbert H, Aschoff A, Ostwald J (1991) Topography of projections from the auditory 
cortex to the inferior colliculus in the rat. J Comp Neurol 304: 103-122.

Hestrin S, Nicoll RA, Perkel DJ, Sah P (1990) Analysis of excitatory synaptic action in pyramidal cells using whole-cell recording from rat hippocampal slices. $J$ Physiol 422: 203-225.

Hille B (2001). Potassium channels and chloride channels. In Ionic Channels of Excitable Membranes, ed. Hille B, pp. 131 - 162. Sinauer, Massachusetts.

Hollmann M, Heinemann S (1994) Cloned glutamate receptors. Annu Rev Neurosci 17: 31-108.

Hosomi H, Mori M, Amatsu M, Okada Y (1997) GABA-activated conductance in cultured rat inferior colliculus neurons. J Neurophysiol 77(2):994-1002.

Huffman RF, Henson OWJr (1990) The descending auditory pathway and acousticomotor systems: connections with the inferior colliculus. Brain Res Rev 15: 295-323.

Huguenard JR, Prince DA (1992) A novel T-type current underlies prolonged $\mathrm{Ca} 2+$-dependent burst firing in GABAergic neurons of rat thalamic reticular nucleus. J Neurosci 12: 3804-3817.

Hurley LM, Pollak GD (1999) Serotonin differentially modulates responses to tones and frequency-modulated sweeps in the inferior colliculus. J Neurosci 19: 8071-8082.

Inagaki N, Toda $\mathrm{K}$, Taniuchi I, Panula $\mathrm{P}$, Yamatodani A, Tohyama M, Watanabe T, Wada $H$ (1990) An analysis of histaminergic efferents of the tuberomammillary nucleus to the medial preoptic area and the inferior colliculus in the rat. Exp Brain Res 80: 374-380.

Ingham NJ, McAlpine D (2005) GABAergic inhibition controls neural gain in inferior colliculus neurons sensitive to interaural differences. $J$ Neurosci 25:6187-6198.

Irfan N, Zhang H, Wu SH (2005) Synaptic transmission mediated by ionotropic glutamate, glycine and GABA receptors in the rat's ventral nucleus of the lateral lemniscus. Hear Res 203: 159-171.

Irvine DR, Gago G (1990) Binauralinteraction in high-frequency neurons in inferior Colliculus of the cat: effects of variations in sound pressure level on sensitivity to interaural intensity differences. J Neurophysiol 63: 570-591.

Irvine DRF. The auditory brain stem: a review of the structure and function of auditory brain stem processing mechanisms. In: Progress in Sensory Physiology, edited by Ottoson D. Berlin: Springer-Verlag, 1986, vol. 7, p.1-279.

Ishida $Y$, Nakahara D, Hashigughi H, Nakamura M, Ebihara K, Takeda R, Nishimori T, Niki H (2002) Fos expression in GABAergic cells and cells immunopositive for NMDA receptors in the inferior and superior colliculi following audiogenic seizures in rats. Synapse 46: 100-107. 
Iwada J, LeDoux JE, Meeley MP, Arneric S, Reis DJ (1986) Intrinsic neurons in the amygdaloid field projected to by the medial geniculate body mediate emotional responses conditioned to acoustic stimuli. Brain Res 383: 195-214.

Jacobs B, Fornal C (1999) Activity of serotonergic neurons in behaving animals. Neuropsychopharmacol 21: 9-15.

Jane JA, Masterton RB, Diamond IT (1965) The function of the tectum for attention to auditory stimuli in the cat. $J$ Comp Neurol 125: 165-192.

Jen PH-S, Chen QC, Sun XD (1998) Corticofugal regulation of auditory sensitivity in the bat inferior colliculus. J Comp Physiol (A) 183: 683-697.

Jen PH-S, Sun X, Chen QC (2001) An electrophysiological study on neural pathways for corticofugally inhibited neurons in the central nucleus of the inferior colliculus of the big brown bat, Eptesicus fuscus. Exp Brain Res 137: 292-302.

Jenkins WM, Masterton RB (1982) Sound localization: effects of unilateral lesions in central auditory system. $J$ Neurophysiol 47(6):987-1016.

Jensen FE (1999) Acute and chronic effects of seizures in the developing brain: experimental models. Epilepsia 40 (Suppl 1): S51-S58.

Jensen K, Chiu CS, Sokolova I, Lester HA, Mody I (2003) GABA transporter-1 (GAT1)-deficient mice: differential tonic activation of $\mathrm{GABA}_{\mathrm{A}}$ versus $\mathrm{GABA}_{\mathrm{B}}$ receptors in the hippocampus. $J$ Neurophysiol 90(4):2690-701.

Jiang ZG, Pessia M, North RA (1993) Dopamine and baclofen inhibit the hyperpolarization-activated cation current in rat ventral tegmental neurones. $J$ Physiol 462:753-64.

Johnson RG, Stevens KE, Rose GM (1998) 5-HT2 receptors modulate auditory filtering in the rat. $J$ Pharmacol Exp Ther 285: 643-650.

Jones KA, Borowsky B, Tamm JA (1998) GABAb receptors function as a heteromeric assembly of the subunits GABAbR1 and GABAbR2. Nature 396: 674-679.

Jourdain P, Poulain DA, Theodosis DT, Israel JM (1996) Electrical properties of oxytocin neurons in organotypic cultures from postnatal rat hypothalamus. $J$ Neurophysiol 76(4):2772-85.

Kamondi A, Reiner PB (1991) Hyperpolarization-activated inward current in histaminergic tuberomammillary neurons of the rat hypothalamus. $J$ Neurophysiol 66: 1902-1911.

Kandel ER, Schwartz JH, Jessell TM (2000) Principles of Neural Science. McGraw-Hill Professional Publishing, UCSF.

Kandler K, Friauf E (1995) Development of glycinergic and glutamatergic synaptic transmission in the auditory brainstem of perinatal rats. J Neurosci 15: 6890-6904.

Kaneda K, Kita H (2005) Synaptically released GABA activates both pre- and 
postsynaptic GABA(B) receptors in the rat globus pallidus. $J$ Neurophysiol 94(2):1104-14.

Kanold DO, Manis PB (1999) Transient K+ currents regulate the discharge patterns of dorsal cochlear nucleus pyramidal cells. $J$ Neurosci 19: 2195-2208.

Kanold PO, Manis PB (2001) A physiologically based model of discharge pattern regulation by transient $\mathrm{K}+$ currents in cochlear nucleus pyramidal cells. $J$ Neurophysiol 85: 523-538.

Kauer J (1992) Whole-cell patch clamp recording method in brain slice preparations. Society for Neuroscience pp 1-9.

Kaupmann K, Malitschek B, Schuler V, Heid J, Froestl W, Beck P, Mosbacher J, Bischoff S, Kulik A, Shigemoto R, Karschin A, Bettler B (1998) GABA(B)-receptor subtypes assemble into functional heteromeric complexes. Nature 396(6712): 683-7.

Keller BU, Konnerth A, Yaari Y (1991) Patch clamp analysis of excitatory synaptic currents in granule cells of rat hippocampus. $J$ Physiol 435: 275-293.

Kelly JB, Caspary DM (2005) Pharmacology of the inferior colliculus. In: The Inferior Colliculus (Winer JA, Schreiner CE, eds) pp 248-281 New York: Springer-Verlag.

Kelly JB, Zhang H (2002) Contribution of AMPA and NMDA receptors to excitatory responses in the inferior colliculus. Hear Res 168: 35-42.

Kim DO, Ghoshal S, Khant SL, Parham K (1994) A computational model with ionic conductances for the fusiform cell of the dorsal cochlear nucleus. $J$ Acoust Soc Am 96:1501-1514.

Klepper A, Herbert H (1991) Distribution and origin of noradrenergic and serotonergic fibers in the cochlear nucleus and inferior Colliculus of the rat. Brain Res 557: 190-201.

Klug A, Park TJ, Pollak GD (1995) Glycine and GABA influence binaural processing in the inferior colliculus of the mustache bat. J Neurophysiol 74: 1701-1713.

Koch U, Grothe B (1998) GABAergic and glycinergic inhibition sharpen tuning for frequency modulations in the inferior colliculus of the big brown bat. $J$ Neurophysiol 80: 71-82.

Koch U, Grothe B (2000) Interdependence of spatial and temporal coding in the auditory midbrain. J Neurophysiol 83(4):2300-14.

Koch U, Grothe B (2003) Hyperpolarization-activated current (Ih) in the inferior colliculus: distribution and contribution to temporal processing. $J$ Neurophysiol 90(6):3679-87.

Koester J, Siegelbaum SA (2000) Local signaling: passive electrical properties of the 
neuron. Pp 140-149. In. Principles of Neural Science. 4ed. Kandel, E.R., Schwartz, J.H., Jessel, T.M. Eds. McGraw Hill.

Kolaj M, Bai D, Renaud LP (2004) $\mathrm{GABA}_{\mathrm{B}}$ receptor modulation of rapid inhibitory and excitatory neurotransmission from subfornical organ and other afferents to median preoptic nucleus neurons. $J$ Neurophysiol 92(1):111-22.

Kraushaar U, Backus KH (2002) Characterization of GABA(A) and glycine receptors in neurons of the developing rat inferior colliculus. Pflugers Arch 445(2):279-88.

Kubo Y, Baldwin TJ, Jan YN, Jan LY (1993) Primary structure and functional expression of a mouse inward rectifier potassium channel. Nature 362(6416): 127-33.

Kubo Y, Reuveny E, Slesinger PA, Jan YN, Jan LY (1993) Primary structure and functional expression of a rat G-protein-coupled muscarinic potassium channel. Nature 364: 802-806.

Kudo M, Niimi K (1980) Ascending projections of the inferior colliculus in the cat: An autoradiographic study. J Comp Neurol 191: 545-556.

Kulik A, Vida I, Lujan R, Haas CA, Lopez-Bendito G, Shigemoto R, Frotscher M (2003) Subcellular localization of metabotropic $G_{A B A}$ receptor subunits $\mathrm{GABA}_{\mathrm{B} 1 \mathrm{a} / \mathrm{b}}$ and $\mathrm{GABA}_{\mathrm{B} 2}$ in the rat hippocampus. $J$ Neurosci $23(35)$ : 11026-35.

Kuner R, Kohr G, Grunewald S, Eisenhardt G, Bach A, Kornau HC (1999) Role of heteromer formation in GABAB receptor function. Science 283(5398): 74-7.

Kuwada S, Batra R (1999) Coding of sound envelopes by inhibitory rebound in neurons of the superior olivary complex in the unanesthetized rabbit. $J$ Neurosci 19, 2273-2287.

Kuwada S, Batra R, Yin TC, Oliver DL, Haberly LB, Stanford TR (1997) Intracellular recordings in response to monaural and binaural stimulation of neurons in the inferior colliculus of the cat. $J$ Neurosci 17:7565-7581.

Kuypers HGJM, Lawrence DG (1967) Cortical projection to the red nucleus and the brain stem in the rhesus monkey. Brain Res 4: 151-188.

Lambert NA, Wilson WA (1993) Discrimination of post- and presynaptic GABA receptor-mediated responses by tetrahydroaminoacridine in area $\mathrm{CA} 3$ of the rat hippocampus. J Neurophysiol 69(2): 630-5.

Lambert NA, Wilson WA (1996) High-threshold $\mathrm{Ca}^{2+}$ currents in rat hippocampal interneurones and their selective inhibition by activation of GABA(B) receptors. $J$ Physiol 492 (Pt 1):115-27.

Large EW, Crawford JD (2002) Auditory temporal computation: Interval selectivity based on post-inhibitory rebound. J Computation Neurosci 13: 125-142.

LeBeau FEN, Rees A, Malmierca MS (1996) Contribution of GABA- and 
glycine-mediated inhibition to the monaural temporal response properties of neurons in the inferior colliculus. $J$ Neurophysiol 75: 902-919.

LeBeau FE, Malmierca MS, Rees A (2001) Iontophoresis in vivo demonstrates a key role for $\mathrm{GABA}(\mathrm{A})$ and glycinergic inhibition in shaping frequency response areas in the inferior colliculus of guinea pig. $J$ Neurosci $21(18): 7303-12$.

Ledoux JE, Iwata J, Cicchetti P, Reis DJ (1988) Different projections of the central amygdaloid nucleus mediate autonomic and behavioural correlates of conditioned fear. J Neurosci 8: 2517-2529.

Lei S, McBain CJ (2003) $\mathrm{GABA}_{\mathrm{B}}$ receptor modulation of excitatory and inhibitory synaptic transmission onto rat CA3 hippocampal interneurons. $J$ Physiol 546: 439-53.

Li L, Kelly JB (1992) Inhibitory influence of the dorsal nucleus of the lateral lemniscus on binaural responses in the rat's inferior colliculus. J Neurosci $12(11)$ : 4530-4539.

Li QH, Nakadate K, Tanaka-Nakadate S, Nakatsuka D, Cui Y, Watanabe Y (2004) Unique expression patterns of 5-HT2A and 5-HT2C receptors in the rat brain during postnatal development: Western blot and immunohistochemical analyses. $J$ Comp Neurol 469: 128-140.

Li X, Bennett DJ (2007) Apaminn-sensitive calcium-activated potassium currents (SK) are activated by persistent calcium currents in rat motoneurons. $J$ Neurophysiol 36 : 3314-3330.

Li Y, Evans S, Faingold CL (1998) In vitro electrophysiology of neurons in subnuclei of rat inferior colliculus. Hear Res 121: 1-10.

Li Y, Evans S, Faingold CL (1999) Synaptic response patterns of neurons in the cortex of rat inferior colliculus. Hear Res 137: 15-28.

Li Y, Stern JE (2004) Activation of postsynaptic GABA $B$ receptors modulate the firing activity of supraoptic oxytocin and vasopressin neurones: role of calcium channels. J Neuroendocrinol 16(2):119-30.

Liu X, Leung LS (2003) Partial hippocampal kindling increases $\mathrm{GABA}_{\mathbf{B}}$ receptor-mediated postsynaptic currents in CA1 pyramidal cells. Epilepsy Res 57(1):33-47.

Ma CL, Kelly JB, Wu SH (2002a) Presynaptic modulation of GABAergic inhibition by $\mathrm{GABA}_{\mathrm{B}}$ receptors in the rat's inferior colliculus. Neuroscience 114(1): 207-215.

Ma CL, Kelly JB, Wu SH (2002b) AMPA and NMDA receptors mediate synaptic excitation in the rat's inferior colliculus. Hear Res 168: 25-34.

Maccaferri G, McBain CJ (1996) The hyperpolarization-activated current $\left(\mathrm{I}_{h}\right)$ and its contribution to pacemaker activity in rat CA1 hippocampal stratum oriens-alveus 
interneurones. $J$ Physiol 497 (Pt 1):119-30.

Maccaferri M, Mangoni M, Lazzari A, DiFrancesco D (1993) Properties of the hyperpolarization-activated current in rat hippocampal CA1 pyramidal cells. $J$ Neurophys 69: 2129-2136.

Magnusson AK, Kapfer C, Grothe B, Koch U (2005) Maturation of glycinergic inhibition in the gerbil medial superior olive after hearing onset. $J$ Physiol $568: 497-512$.

Malitschek B, Ruegg D, Heid J, Kaupmann K, Bittiger H, Frostl W, Bettler B, Kuhn $R$ (1998) Developmental changes of agonist affinity at $G A B A_{B} R 1$ receptor variants in rat brain. Mol Cell Neurosci 12(1-2):56-64.

Malmierca MS (2003) The structure and physiology of the rat auditory system: an overview. Int Rev Neurobiol 56:147-211.

Malmierca MS, Blackstad TW, Osen KK, Karagülle T, Molowny RL (1993) The central nucleus of the inferior colliculus in rat: a Golgi and computer reconstruction study of neuronal and laminar structure. J Comp Neurol 333(1): $1-27$.

Malmierca MS, Le Beau FE, Rees A (1996) The topographical organization of descending projections from the central nucleus of the inferior colliculus in guinea pig. Hear Res 93(1-2):167-80.

Malmierca MS, Leergaard TB, Bajo VM, Bjaalie JG, Merchán MA (1998) Anatomic evidence of a three-dimensional mosaic pattern of tonotopic organization in the ventral complex of the lateral lemniscus in cat. J Neurosci 18(24):10603-18

Malmierca MS, Rees A, Le Beau FE, Bjaalie JG (1995) Laminar organization of frequency-defined local axons within and between the inferior colliculi of the guinea pig. J Comp Neurol 357(1):124-44.

Malmierca MS: Computer-assisted 3-D reconstructions of Golgi-impregnated cells in the rat inferior colliculus; thesis, University of Oslo \& Salamanca, 1991.

Manis PB (1990) Membrane properties and discharge characteristics of guinea pig dorsal cochlear nucleus neurons studied in vitro. J Neurosci 10:2338-2351.

Matsushima T, Tegnér J, Hill RH, Grillner S (1993) GABA B $_{B}$ receptor activation causes a depression of low- and high-voltage-activated $\mathrm{Ca}^{2+}$ currents, postinhibitory rebound, and postspike afterhyperpolarization in lamprey neurons. $J$ Neurophysiol 70(6):2606-19.

Mayer ML, Westbrook GL (1983) A voltage-clamp analysis of inward (anomalous) rectification in mouse spinal sensory ganglion neurons. $J$ Physiol (Lond.) 340: $19-45$.

Mayer ML, Westbrook GL. Permeation and block of N-methyl-D-aspartic acid receptor channels by divalent cations in mouse cultured central neurones. $J$ 
Physiol 394:501-27.

McCormick DA, Pape HC (1990) Properties of a hyperpolarization-activated cation current and its role in rhythmic oscillation in thalamic relay neurons. $J$ Physiol 431: 291-318.

McCown TJ, Breese GR (1991) Seizure interactions between the inferior collicular cortex and the deep prepiriform cortex. Epilepsy Res 8: 21-29.

McCown TJ, Greenwood RS, Frye GD, Breese GR (1984) Electrically elicited seizures from the inferior colliculus: A potential site for the genesis of epilepsy? Exp Neurol 86: 527-542.

Meech RW (1978) Calcium-dependent potassium activation in nervous tissues. Annu Rev Biophys Bioeng 7:1-18.

Melo LL, Brandão ML (1995) Role of 5-HT1A and 5-HT2 receptors in the aversion induced by electrical stimulation of inferior colliculus. Pharm Biochem Behav 51: 317-321.

Merchan M, Aguilar LA, Lopez-Poveda EA, Malmierca MS (2005) The inferior colliculus of the rat: quantitative immunocytochemical study of GABA and glycine. Neurosci 136: 907-925.

Mercuri NB, Bonci A, Calabresi P, Stefani A, Bernardi G (1995) Properties of the hyperpolarization-activated cation current in rat midbrain dopaminergic neurons. Eur J Neurosci 7: 462-469.

Merzenich MM, Reid MD (1974) Representation of the cochlea within the inferior colliculus of the cat. Brain Res 77: 397-415.

Milbrandt JC, Albin RL, Caspary DM (1994) Age-related decrease in GABA receptor binding in the Fischer 344 rat inferior colliculus. Neurobiol Aging 15: 699-703.

Milbrandt JC, Albin RL, Turgeon SM, Caspary DM (1996) GABA A $_{\text {receptor binding }}$ in the aging rat inferior colliculus. Neuroscience 73: 449-458.

Milbrandt JC, Hunter C, Caspary DM (1997) Alterations of $\mathrm{GABA}_{\mathrm{A}}$ receptor subunit mRNAs levels in the aged Fischer 344 rat inferior colliculus. J Comp Neurol 379: 455-465.

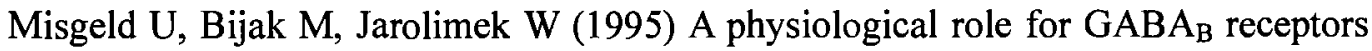
and the effects of baclofen in the mammalian central nervous system. Prog Neurobiol 46(4):423-462.

Mishra PK, Dailey JW, Reigel CE, Tomsic ML, Jobe PC (1988) Sex-specific distinctions in audiogenic convulsions exhibited by severe seizure genetically epilepsy-prone rats (GEPR-9s). Epilepsy Res 2: 309-316.

Molineux ML, Fernandez FR, Hamish Mehaffey W, Turner RW (2005) A-type and 
T-type currents interact to produce a novel spike latency-voltage relationship in cerebellar stellate cells. $J$ Neurosci 25:10863-10873.

Monyer H, Sprengel R, Schoepfer R, Herb A, Higuchi M, Lomeli H, Burnashev N, Sakmann B, Seeburg PH (1992) Heteromeric NMDA receptors: molecular and functional distinction of subtypes. Science 256:1217-1221.

Moore JK, Moore RY (1987) Glutamic acid decarboxylase-like immunoreactivity in brainstem auditory nuclei of the rat. $J$ Comp Neurol 260: 157-174.

Moore RY, Goldberg JM (1963) Ascending projections of the inferior colliculus in the cat. J Comp Neurol 121: 109-135.

Moore RY, Goldberg JM (1966) Projections of the inferior colliculus in the monkey. Exp Neurol 14: 429-438.

Moore, David R., Vibhakar C. Kotak, and Dan H. Sanes (1998) Commissural and lemniscal synaptic input to the gerbil inferior colliculus. $J$ Neurophysiol 80: 2229-2236.

Morest DK (1966) The cortical structure of the inferior quadrigeminal lamina of the cat. Anat Rec 154: 289-390.

Morest DK, Oliver DL (1984) The neuronal architecture of the inferior colliculus in the cat defining the functional anatomy of the auditory midbrain. J Comp Neurol 222: 209-236.

Morest DK, Winer JA (1986) The comparative anatomy of neurons: homologous neurons in the medial geniculate body of the opossum and the cat. Adv Anat Embryol Cell Biol 97: 1-94.

Morrisett RA, Mott DD, Lewis DV, Swartzwelder HS, Wilson WA (1991) GABAB-receptor mediated inhibition of the N-methyl-D-aspartate component of synaptic transmission in the rat hippocampus. J Neurosci 11: 203-209.

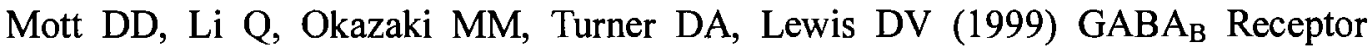
mediated currents in interneurons of the dentate-hilus border. $J$ Neurophysiol 82(3):1438-50.

N'gouemo P, Morad M (2003) Voltage-gated calcium channels in adult rat inferior colliculus neurons. Neurosci 120: 815-826.

N'gouemo P, Rittenhouse AR (2000) Biophysical and pharmacological characterization of voltage-sensitive calcium currents in neonatal rat inferior colliculus neurons. Neurosci 96: 753-765.

Nataraj K, Wenstrup JJ (2005) Roles of inhibition in creating complex auditory responses in the inferior colliculus: facilitated combination-sensitive neurons. $J$ Neurophysiol 93: 3294-3312.

Nataraj K, Wenstrup JJ (2006) Roles of inhibition in complex auditory responses in 
the inferior colliculus: inhibited combination-sensitive neurons. $J$ Neurophysiol 95:2179-2192.

Nayagam DAX, Clarey JC, Paolini AG (2005) Powerful, onset inhibition in the ventral nucleus of the lateral lemniscus. J Neurophysiol 94:1651-1654.

Neher E (1971) Two fast transient current components during voltage clamp on snail neurons. J Gen Physiol 58:36-53.

Neher E (1992) Correction for liquid junction potentials in patch clamp experiments. Meth Enzymol 207:123-131.

Ng GY, Clark J, Coulombe N, Ethier N, Hebert TE, Sullivan R, Kargman S, Chateauneuf A, Tsukamoto N, McDonald T, Whiting P, Mezey E, Johnson MP, Liu Q, Kolakowski LF Jr, Evans JF, Bonner TI, O'Neill GP (1999) Identification of a $\mathrm{GABAB}$ receptor subunit, gb2, required for functional $\mathrm{GABA}_{\mathrm{B}}$ receptor activity. $J$ Biol Chem 274(12):7607-10.

Nichols CG, Lopatin AN (1997) Inward rectifier potassium channels. Annu Rev Physiol 59: 171-191.

Nicoll RA (1988) The coupling of neurotransmitter receptors to ion channels in the brain. Science 241(4865): 545-51.

Nitzan R, Segev I, Yarom Y (1990) Voltage behavior along the irregular dendritic structure of morphologically and physiologically characterized vagal motoneurons in the guinea pig. $J$ Neurophysiol 63: 333-346.

Nowak L, Bregestovski P, Ascher P, Herbet A, Prochiantz A (1984) Magnesium gates glutamate-activated channels in mouse central neurones. Nature 307(5950): $462-5$.

Numann RE, Wadman WJ, Wong RK (1987) Outward currents of single hippocampal cells obtained from the adult guinea-pig. J Physiol 393:331-53.

Oertel D (1999) The role of timing in the brain stem auditory nuclei of vertebrates. Annu Rev Physiol 61:497-519.

Olazábal UE, Moore JK (1989) Nigrotectal projection to the inferior colliculus: horseradish peroxidase transport and tyrosine hydroxylase immunohistochemical studies in rats, cats, and bats. J Comp Neurol 282(1):98-118.

Oliver DL (1984) Neuron types in the central nucleus of the inferior colliculus that project to the medial geniculate body. Neurosci 11: 409-424.

Oliver DL (1987) Projections to the inferior colliculus from the anteroventral cochlear nucleus in the cat: possible substrates for binaural interaction. $J$ Comp Neurol 264(1): 24-46.

Oliver DL (2005) Neuronal organization in the inferior colliculus. In: The Inferior Colliculus, (Winer JA and Schreiner CE, eds) pp 69-114 New York: 
Springer-Verlag.

Oliver DL, Hall WC (1978) The medial geniculate body of the tree shrew, Tupaia glis.

II. Connections with the neocortex. J Comp Neurol 182(3):459-93.

Otis TS, Mody I (1992) Differential activation of $\mathrm{GABA}_{\mathrm{A}}$ and $\mathrm{GABA}_{\mathrm{B}}$ receptors by spontaneously released transmitter. $J$ Neurophysiol 67: 227-235.

Overstreet LS, Westbrook GL (2001) Paradoxical reduction of synaptic inhibition by vigabatrin. $J$ Neurophysiol 86(2): 596-603.

Ozawa S, lino M, Abe M (1991) Excitatory synapse in the rat hippocampus in tissue culture and effects of aniracetam. Neurosci Res 12: 72-82.

Pagano A, Rovelli G, Mosbacher J, Lohmann T, Duthey B, Stauffer D, Ristig D, Schuler V, Meigel I, Lampert C, Stein T, Prezeau L, Blahos J, Pin J, Froestl W, Kuhn R, Heid J, Kaupmann K, Bettler B (2001) C-terminal interaction is essential for surfacetrafficking but not for heteromeric assembly of $\mathrm{GABA}_{\mathrm{B}}$ receptors. $J$. Neurosci 21, 1189-1202.

Palombi PS, Backoff PM, Caspary DM (2001) Responses of young and aged rat inferior colliculus neurons to sinusoidally amplitude modulated stimuli. Hear Res 153: 174-180.

Palombi PS, Caspary DM (1996) GABA inputs control discharge rate primarily within frequency receptive fields of inferior colliculus neurons. $J$ Neurophysiol 75(6): 2211-9.

Paolini AG Clarey JC, Needham K, Clark GM (2004) Fast inhibition alters first spike timing in auditory brainstem neurons. $J$ Neurophysiol 92: 2615-2621.

Pape HC (1996) Queer current and pacemaker: the hyperpolarization-activated cation current in neurons. Annu Rev Physiol 58: 299-327.

Park TJ, Pollak DG (1993) GABA shapes sensitivity to interaural intensity disparities in the mustache bat's inferior colliculus: implications for encoding sound location. $J$ Neurosci 13(5):2050-67.

Park TJ, Pollak GD (1994) Azimuthal receptive fields are shaped by GABAergic inhibition in the inferior colliculus of the mustache bat. $J$ Neurophysiol 72(3):1080-102.

Parks TN (2000) The AMPA receptors of auditory neurons. Hear Res 147(1-2):77-91.

Paxinos G (1999) Chemoarchitectonic Atlas of the rat Brainstem. Academic Press, San Diego.

Paxinos G Watson C (1998) The rat brain in stereotaxic coordinates (4th ed.), Academic Press, San Diego.

Peres-Reyes E (2003) Molecular physiology of low voltage-activated T-type calcium channels. Physiol Rev 83: 117-161. 
Pérez-González D, Malmierca MS, Covey E (2005) Novelty detector neurons in the mammalian auditory midbrain. Eur J Neurosci 22: 2879-2885.

Pérez-González D, Malmierca MS, Moore JM, Hernández O, Covey E (2006) Duration selective neurons in the inferior colliculus of the rat: topographic distribution and relation of duration sensitivity to other response properties. $J$ Neurophysiol 95:823-836.

Peroutka SJ (1994) Molecular biology of serotonin (5-HT) receptor. Synapse 18: 241-260.

Peruzzi D, Bartlett E, Smith PH, Oliver DL (1997) A monosynaptic GABAergic input from the inferior colliculus to the medial geniculate body in rat. $J$ Neurosci 17(10):3766-77.

Peruzzi D, Sivaramakrishna S, Oliver DL (2000) Identification of cell types in brain slices of the inferior colliculus. Neurosci 101:403-416.

Petralia RS, Wenthold RJ (1992) Light and electron immunocytochemical localization of AMPA-selective glutamate receptors in the rat brain. J Comp Neurol 318(3):329-54.

Phelan KD (1999) N-ethylmaleimide selectively blocks presynaptic GABA autoreceptor but not heteroreceptor mediated inhibition in adult rat striatal slices. Brain Res 847:308-313.

Pierson M, Snyder-Keller A. (1994) Development of frequency-selective domains in inferior colliculus of normal and neonatally noise-exposed rats. Brain Res 636(1):55-67.

Pierson MG, Swann J (1991) Ontogenetic features of audiogenic seizure susceptibility induced in immature rats by noise. Epilepsia 32: 1-9.

Pollak GD, Burger RM, Klug A (2003) Dissecting the circuitry of the auditory system. Trends Neurosci 26(1): 33-9.

Pollo A, Lovallo M, Sher E, Carbone E (1992) Voltage-dependent noradrenergic modulation of omega-conotoxin-sensitive $\mathrm{Ca}^{2+}$ channels in human neuroblastoma IMR32 cells. Pflugers Arch 422(1):75-83.

Poorkhalkali N, Juneblad K, Jonsson AC, Lindberg M, Karlsson O, Wallbrandt P, Ekstrand J, Lehmann A (2001) Immunocytochemical distribution of the GABA(B) receptor splice variants $\mathrm{GABA}(\mathrm{B}) \mathrm{R} 1 \mathrm{a}$ and $\mathrm{R} 1 \mathrm{~b}$ in the rat CNS and dorsal root ganglia. Anat Embryol (Berl) 201(1):1-13.

Potier B, Dutar P (1993) Presynaptic inhibitory effect of baclofen on hippocampal inhibitory synaptic transmission involves a pertussis toxin-sensitive G-protein. Eur J Pharmacol 231(3): 427-33.

Premkumar LS, Gage PW (1994) Potassium channels activated by GABAB agonists and serotonin in cultured hippocampal neurons. J Neurophysiol 71(6):2570-5. 
Prince DA (1985) Physiological mechanisms of focal epileptogenesis. Epilepsia 26[suppl 1]: S3-S14.

Princivalle AP, Duncan JS, Thom M, Bowery NG (2002) Studies of GABA(B) receptors labelled with [(3)H]-CGP62349 in hippocampus resected from patients with temporal lobe epilepsy. Br J Pharmacol 136: 1099-1106.

Rabow LE, Russek SJ, and Farb DH (1995) From ion currents to genomic analysis: recent advances in GABAA receptor research. Synapse 21: 189-274.

Rees A, Sarbaz A, Malmierca MS, Le Beau FEN (1997) Regularity of firing of neurons in the inferior colliculus. J Neurophysiol 77: 2945-2965.

Reid HM, Collins RL (1989) Independence of uniphasic and biphasic audiogenic seizure progressions in mice. Behav Neural Biol 52: 417-421.

Rhode WS, Smith PH, Oertel D (1983) Physiological response properties of cells labeled intracellularly with horseradish peroxidase in cat dorsal cochlear nucleus. J Comp Neurol 213: 426-447.

Ribak CE, Khurana V, Lien NT (1994) The effect of midbrain follicular knife cuts on audiogenic seizure severity in the genetically epilepsy-prone rat. $J$ Hirnforsch 35 : 303-311.

Rinzel J, Terman D, Wang X-J, Ermentrout B (1998) Propagating activity patterns in large-scale inhibitory neuronal networks. Science 279:1351-1355.

Rockel AJ, Jones EG (1973) Observations on the fine structure of the central nucleus of the inferior colliculus of the cat. J Comp Neurol 147: 61-92.

Roger M, Arnault P (1989) Anatomical study of the connections of the primary auditory area in the rat. J Comp Neurol 287: 339-356.

Rosenberger MH, Fremouw T, Casseday JH, Covey E (2003) Expression of the Kv1.1 ion channel subunit in the auditory brainstem of the big brown bat, Eptesicus fuscus. J Comp Neurol 462:101-120.

Ross KC, Coleman JR (2000) Developmental and genetic audiogenic seizure models: behaviour and biological substrates. Neurosci Biobehav Rev 24: 639-653.

Russier M, Carlier E, Ankri N, Fronzaroli L, Debanne D (2003) A-, T-, and H-type currents shape intrinsic firing of developing rat abducens motoneurons. $J$ Physiol 549:21-36.

Russo MJ, Mugnaini E, Martin M (2007) Intrinsic properties and mechanisms of spontaneous firing in mouse cerebellar unipolar brush cells. $J$ Physiol 581:709-724.

Ryu PD, Randic M (1990) Low- and high-voltage-activated calcium currents in rat spinal dorsal horn neurons. J Neurophysiol 63: 272-285.

Ryugo DK, Willard FH, Fekete DM (1981) Differential afferent projections to the 
inferior colliculus from the cochlear nucleus in the albino mouse. Brain Res 210: 342-349.

Sah P (1996) $\mathrm{Ca}^{2+}$-activated $\mathrm{K}^{+}$currents in neurones: types, physiological roles and modulation. Trends Neurosci 19(4):150-154.

Saint Marie RL (1996) Glutamatergic connections of the auditory midbrain: selective uptake and axonal transport of D-[3H]aspartate. J Comp Neurol 373(2):255-70.

Sakamoto T, Niki H (2001) Acoustic priming lowers the threshold for electrically induced seizures in mice inferior colliculus, but not in the deep layers of superior colliculus. Brain Res 898: 358-363.

Saldaňa E, Merchán MA (1992) Intrinsic and commissural connections of the rat inferior colliculus. J Comp Neurol 319:417-37.

Saldan̆a E, Merchán MA (2005) Intrinsic and commissural connections of the inferior colliculus. In: The Inferior Colliculus, (Winer JA and Schreiner CE, eds) pp 155-181 New York: Springer-Verlag.

Sato K, Shiraishi S, Nakagawa H, Kuriyama H, Altschuler RA (2000) Diversity and plasticity in amino acid receptor subunits in the rat auditory brain stem. Hear Res 147: 137-144.

Schofield BR (2005) Superior Olivary Complex and Lateral Lemniscal Connections of the Auditory Midbrain. In: The Inferior Colliculus, (Winer JA and Schreiner CE, eds) pp 132-154 New York: Springer-Verlag.

Schweitzer P, Madamba SG, Siggins GR (2004) The sleep-modulating peptide cortistatin augments the h-current in hippocampal neurons. $J$ Neurosci 23(34): 10884-91.

Scott LL, Mathews PJ, Golding NL (2005) Posthearing developmental refinement of temporal processing in principal neurons of the medial superior olive. $J$ Neurosci 25:7887-7895.

Seeburg PH (1993) The molecular biology of mammalian glutamate receptor channels. Trends Neurosci 16(9):359-65.

Sekirnjak C, du Lac S (2002) Intrinsic firing dynamics of vestibular nucleus neurons. $J$ Neurosci 22(6):2083-95.

Semple MN, Kitzes LM (1987) Binaural processing of sound pressure level in the inferior Colliculus. $J$ Neurophysiol 57: 1130-1147.

Shannon RV, Zeng FG, Kamath V, Wygonski J, Ekelid M (1995) Speech recognition with primarily temporal cues. Science 270(5234):303-4.

Sheng M, Cummings J, Roldan LA, Jan YN, Jan LY (1994). Changing subunit composition of heteromeric NMDA receptors during development of rat cortex. Nature 368, 144-147. 
Sherman-Gold R (1993) The axon guide for electrophysiology and biophysics laboratory techniques. Axon Instruments Inc. pp 55-116.

Shiraishi S, Shiraishi Y, Oliver DL, Altschuler RA (2001) Expression of GABA(A) receptor subunits in the rat central nucleus of the inferior colliculus. Brain Res Mol Brain Res 96(1-2):122-32.

Shneiderman A, Chase MB, Rockwood JM, Benson CG, Potashner SJ (1993) Evidence for a GABAergic projection from the dorsal nucleus of the lateral lemniscus to the inferior colliculus. $J$ Neurochem 60 : 72-82.

Shneiderman A, Oliver DL, Henkel CG (1988) Connections of the dorsal nucleus of the lateral lemniscus: An inhibitory parallel pathway in the ascending auditory system? J Comp Neurol 276: 188-208.

Sivaramakrishna S, Oliver DL (2001) Distinct K currents result in physiologically distinct cell types in the inferior colliculus of the rat. $J$ Neurosci 21:2861-2877.

Sivaramakrishna S, Oliver DL (2006) The generation of rebound excitation in the inferior colliculus. Assoc. Res. Otolaryngol. Abstr. 29: 51-52.

Slugg RM, Zheng SX, Fang Y, Kelly MJ, Rønnekleiv OK (2003) Baclofen inhibits guinea pig magnocellular neurones via activation of an inwardly rectifying $\mathrm{K}^{+}$ conductance. J Physiol 551(Pt 1):295-308.

Smith PH (1992) Anatomy and physiology of multipolar cells in the rat inferior collicular cortex using the in vitro brain slice technique. J Neurosci 12:3700-3715.

Sodickson DL, Bean BP (1998) Neurotransmitter activation of inwardly rectifying potassium current in dissociated hippocampal CA3 neurons: interactions among multiple receptors. $J$ Neurosci 18(20):8153-62.

Spain WJ, Schwindt PC, Crill WE (1987) Anomalous rectification in neurons from cat sensorimotor cortex in vitro. J Neurophysiol 57: 1555-1576.

Spigelman I, Zhang L, Carlen PL (1992) Patch-clamp study of postnatal development of CA1 neurons in rat hippocampal slices: membrane excitability and $\mathrm{K}+$ currents. $J$ Neurophysiol 68(1):55-69.

Stackman RW, Hammond RS, Linardatos E, Gerlach A, Maylie J, Adelman JP, Tzounopoulos $\mathrm{T}$ (2002) Small conductance $\mathrm{Ca}^{2+}$-activated $\mathrm{K}^{+}$channels modulate synaptic plasticity and memory encoding. $J$ Neurosci 22: 10163-10171.

Stiebler I, Ehret G (1985) Inferior colliculus of the house mouse. I. A quantitative study of tonotopic organization, frequency representation, and tone-threshold distribution. J Comp Neurol 238: 65-76.

Stocker M, Krause M, Pedarzani P (1999) An apamin-sensitive Ca2+ -activated K+ current in hippocampal pyramidal neurons. Proc. Natl. Acad. Sci 96:4662-4667.

Storm J (1989) An after-hyperpolarization of medium duration in rat hippocampal 
cells. J Physiol 409: 171-190.

Stutzmann GE, McEwen BS, LeDoux JE (1998) Serotonin modulation of sensory inputs to the lateral amygdale: dependency on corticosterone. $J$ Neurosci 18: 9529-9538.

Sun $\mathrm{H}$, Ma CL, Kelly JB, Wu SH (2006) $\mathrm{GABA}_{\mathrm{B}}$ receptor-mediated presynaptic inhibition of glutamatergic transmission in the inferior colliculus. Neurosci Lett 399(1-2):151-6.

Sun $\mathrm{H}$, Wu SH (2006) Physiological role of postsynaptic GABA $A_{B}$ receptors in the dorsal cortex of the young rat's inferior colliculus. Proceedings of the 29th meeting of the Assoc. Res. Otolaryngol., Baltimore, US., p. 233.

Sun H, Wu SH (2007) Modification of membrane excitability of neurons in the rat's dorsal cortex of the inferior colliculus by preceding hyperpolarization. Neuroscience (in press, Epub ahead of print).

Sun XD, Chen QC, Jen PHS (1996) Corticofugal control of central auditory sensitivity. Neurosci Lett 212: 131-134.

Surges R, Freiman TM, Feuerstein TJ (2004) Input resistance is voltage dependent due to activation of $\mathrm{Ih}$ channels in rat CA1 pyramidal cells. $J$ Neurosci Res 76 : 475-480.

Surges R, Sarvari M, Steffens M, Els T (2006) Characterization of rebound depolarization in hippocampal neurons. Biochem Biophys Res Commun 348: 1343-1349.

Syka J, Popelar J (1984) inferior colliculus in the rat: neuronal responses to stimulation of the auditory cortex. Neurosci Lett 51: 235-240.

Syka J, Popelar J, Kvasnak E, Astl J (2000) Response properties of neurons in the central nucleus and external and dorsal cortices of the inferior colliculus in guinea pig. Exp Brain Res 133: 254-266.

Szczepaniak WS, Møller AR (1996) Effects of (-)-baclofen, clonazepam, and diazepam on tone exposure-induced hyperexcitability of the inferior colliculus in the rat: possible therapeutic implications for pharmacological management of tinnitus and hyperacusis. Hear Res 97(1-2):46-53.

Takahashi T, Kajikawa Y, Tsujimoto T (1998) G-Protein-coupled modulation of presynaptic calcium currents and transmitter release by a $\mathrm{GABA}_{\mathrm{B}}$ receptor. $J$ Neurosci 18(9):3138-46.

Takeda M, Tanimoto T, Ikeda M, Kadoi J, Matsumoto S (2004) Activaton of GABA receptor inhibits the excitability of rat small diameter trigeminal root ganglion neurons. Neuroscience 123(2): 491-505.

Takigawa T, Alzheimer C (2003) Interplay between activation of GIRK current and deactivation of Ih modifies temporal integration of excitatory input in CA1 
pyramidal cells. $J$ Neurophysiol 89(4):2238-44.

Tanabe M, Kaneko T (1996) Paired pulse facilitation of GABAergic IPSC's in ventral horn neurons in neonatal spinal cord. Brain Res 716: 101-106.

Tanabe Y, Masu M, Ishii T, Shigemoto R, Nakanishi S (1992) A family of metabotropic glutamate receptors. Neuron 8(1):169-79.

Tennigkeit F, Schwarz DW, Puil E (1996) Mechanisms for signal transformation in lemniscal auditory thalamus. $J$ Neurophysiol 76(6):3597-3608.

Tennigkeit F, Schwarz DW, Puil E (1998) GABA(B) receptor activation changes membrane and filter properties of auditory thalamic neurons. Hear Res 122(1-2):18-24.

Tennigkeit F, Schwarz DWF, Puil E (1998) Postnatal development of signal generation in auditory thalamic neurons. Dev Brain Res 109:255-263.

Teshima K, Kim SH, Allen CN (2003) Characteristics of an apamin-sensitive potassium current in suprachiasmatic nucleus neurons. Neurosci 120: 65-73.

Thompson GC, Thompson AM, Garrett KM, Britton BH (1994) Serotonin and serotonin receptors in the central auditory system. Otolaryngol Head Neck Surg 110: 93-102.

Tokunaga A, Sugita S, Otani K (1984) Auditory and non auditory subcortical afferents to the inferior colliculus in the rat. $J$ Hirnforsch 25 : 461-472.

Towers S, Princivalle A, Billinton A (2000) $\mathrm{GABA}_{\mathrm{B}}$ receptor protein and mRNA distribution in rat spinal cord and dorsal root ganglia. Eur $J$ Neurisci 12(9): 3201-3210.

Trulson ME, Jacobs BL (1979) Raphe unit activity in freely moving cats: correlation with level and behavioural arousal. Brain Res 163: 135-150.

Ulrich D, Huguenard JR (1996) Gamma-aminobutyric acid type B receptor-dependent burst-firing in thalamic neurons: a dynamic clamp study. Proc Natl Acad Sci 93(23):13245-9.

Urban GP, Willot JF (1979) Response properties of neurons in inferior colliculi of mice made susceptible to audiogenic seizures by acoustic priming. Exp Neurol 63: 229-243.

Vale C, Sanes DH (2000) Afferent regulation of inhibitory synaptic transmission in the developing auditory midbrain. J Neurosci $20: 1912-1921$.

Vale C, Schoorlemmer J, Sanes DH (2003) Deafness disrupts chloride transporter function and inhibitory synaptic transmission. $J$ Neurosci 23: 7516-7524.

Vanysek P (1988) Activity coefficients for acids, bases and salts. In: Weast, R.C. (Ed.), Handbook of Chemistry and Physics. CRC Press, Boca Raton, FL, USA, pp. D-169. 
Vargas G Lucero MT (1999) Dopamine modulates inwardly rectifying hyperpolarization-activated current (Ih) in cultured rat olfactory receptor neurons. J Neurophysiol 81: 149-158.

Vater M, Feng AS (1990) Functional organization of ascending and descending connections of the cochlear nucleus of horseshoe bats. J Comp Neurol 292(3): 373-95.

Vaudano E, Legg CR, Glickstein M (1991) Afferent and efferent connections of temporal association cortex in the rat: a horseradish peroxidase study. Eur $J$ Neurosci 3: 317-330.

Vaughn MD, Pozza MF, Lingenhöhl K (1996) Excitatory acoustic responses in the inferior colliculus of the rat are increased by $\mathrm{GABA}_{\mathrm{B}}$ receptor blockade. Neuropharmacology 35(12):1761-7.

Verdoorn TA, Draguhn A, Ymer S, Seeburg PH, Sakmann B (1990) Functional properties of recombinant rat $\mathrm{GABA}_{\mathrm{A}}$ receptors depend upon subunit composition. Neuron 4:919-928.

Viana F, Bayliss DA, Berger AJ (1993) Calcium conductances and their role in the firing behavior of neonatal rat hypoglossal motoneurons. $J$ Neurophysiol 69: 2137-2149.

Vicini S, Wang JF, Li JH, Zhu WJ, Wang YH, Luo JH, Wolfe BB, Grayson DR (1998) Functional and pharmacological differences between recombinant N-methyl-D-aspartate receptors. J Neurophysiol 79: 555-566.

Villalobos C, Shakkottai VG, Chandy KG, Michelhaugh SK, Andrade R (2004) SKca channels mediated the medium but not slow calcium-activated afterhyperpolarization in cortical neurons. $J$ Neurosci 24 : 3537-3542.

Wada JA, Teveo A, White B, Jung E (1970) Inferior colliculus lesion and audiogenic seizure susceptibility. Exp Neurol 28: 326-382.

Wagner T (1994) Intrinsic properties of identified neurons of the central nucleus of mouse inferior colliculus: An intracellular brain slice study. Eur $J$ Neurosci 8: 1231-1239.

Wagner $T$ (1996) Lemniscal input to identified neurons of the central nucleus of mouse inferior colliculus: an intracellular brain slice study. Eur $J$ Neurosci 8: 1231-1239.

Wang Y, Ramage AG, Jordan D (1996) Mediation by 5HT3 receptors of an excitatory effect of 5-HT on dorsal vagal preganglionic neurons in anaesthetized rats: an ionophoretic study. Br J Pharmacol 118: 1697-1704.

Watts AE, Williams JT, Henderson G (1996) Baclofen inhibition of the hyperpolarization-activated cation current, Ih, in rat substantia nigra zona compacta neurons may be secondary to potassium current activation. $J$ 
Neurophysiol 76(4):2262-70.

Weedman DL, Ryugo DK (1996) Pyramidal cells in primary auditory cortex project to cochlear nucleus in rat. Brain Res 706(1):97-102.

Wenstrup JJ, Fuzessery ZM, Pollak GD (1988) Binaural neurons in the mustache bat's inferior colliculus. I. Responses of $60-\mathrm{kHz}$ EI units to dichotic sound stimulation. $J$ Neurophysiol 60(4):1369-83.

Whitley JM, Henkel CK (1984) Topographical organization of the inferior collicular projection and other connections of the ventral nucleus of the lateral lemniscus in the cat. J Comp Neurol 229(2):257-70.

Willard FH, Ho RH, Martin GF (1984) The neuronal types and the distribution of 5-hydroxytryptamine and enkephalin-like immunoreactive fibers in the dorsal cochlear nucleus of the North American opossum. Brain Res Bull 12: 253-266.

Willard FH, Martin GF (1983) The auditory brainstem nuclei and some of their projections to the inferior colliculus in the North American opossum. Neuroscience 10(4):1203-32.

Willard FH, Ryugo DK (1979) External nucleus of the inferior colliculus: A site of overlap for ascending auditory and somatosensory projections in the mouse. Soc Neurosci Abstr 5: 33.

Williams JT, Colmers WF, Pan ZZ (1988). Voltage- and ligand activated inwardly rectifying currents in dorsal raphe neurones in vitro. J Neurosci 8: 3499 - 3506.

Williams SR, Stuart GJ (2000) Site independence of EPSP time course is mediated by dendritic I(h) in neocortical pyramidal neurons. J Neurophysiol 83(5):3177-82.

Willott JF, Lu SM (1982) Noise-induced hearing loss can alter neural coding and increase excitability in the central nervous system. Science 216: 1331-1332.

Winer JA (2005) Three systems of descending projections to the inferior colliculus. In: The Inferior Colliculus (Winer JA and Schreiner CE, eds) pp 231-247 New York: Springer-Verlag.

Winer JA and Schreiner CE (2005) The Central Auditory System: A Functional Analysis. In: The Inferior Colliculus, (Winer JA and Schreiner CE, eds) pp 1-68 New York: Springer-Verlag.

Winer JA, Laru D (2001) Photomicrographs of 867-50 Nissl stained rat brain slices processed by Winer Laboratory June, 2001.

Winer JA, Larue DT, Diehl JJ, Hefti BJ (1998) Auditory cortical projections to the cat inferior colliculus. J Comp Neurol 400(2):147-74.

Winer JA, Prieto JJ (2001) Layer V in cat primary auditory cortex (AI): cellular architecture and identification of projection neurons. J Comp Neurol 434(4): $379-412$. 
Wojcik WJ, Travagli RA, Costa E, Bertolino M (1990) Baclofen inhibits with high affinity an L-type-like voltage-dependent calcium channel in cerebellar granule cell cultures. Neuropharmacology 29(10):969-72.

Woods CI, Azeredo WJ (1999) Noradrenergic and serotonergic projections to the superior olive: potential for modulation of olivocochlear neurons. Brain Res 836: 9-18.

Woollard HH, Harpman JA (1940) The connexions of the inferior colliculus and the dorsal nucleus of the lateral lemniscus. $J$ Anat 74: 441-458.

$\mathrm{Wu} \mathrm{CH}$, Jen $\mathrm{PH}$ (2006) The role of GABAergic inhibition in shaping duration selectivity of bat inferior collicular neurons determined with temporally patterned sound trains. Hear Res 215:56-66.

Wu LG, Saggau P (1997) Presynaptic inhibition of elicited neurotransmitter release. Trends Neurosci 20: 204-212.

Wu RL, Barish ME (1992) Two pharmacologically and kinetically distinct transient potassium currents in cultured embryonic mouse hippocampal neurons. $J$ Neurosci 12(6): 2235-46.

Wu SH (1999) Physiological properties of neurons in the ventral nucleus of the lateral lemniscus of the rat: intrinsic membrane properties and synaptic responses. $J$ Neurophysiol 81:2862-2874.

Wu SH (2005) Biophysical properties of inferior colliculus neurons. In: The Inferior Colliculus (Winer JA, Schreiner CE, eds) pp 282-311. New York: Springer-Verlag.

$\mathrm{Wu}$ SH, Kelly JB (1995) In vitro brain slice studies of the rat's dorsal nucleus of the lateral lemniscus. I. Membrane and synaptic response properties. J Neurophys 73 : 780-793.

Wu SH, Kelly JB (1996) In vitro brain slice studies of the rat's dorsal nucleus of the lateral lemniscus. II. Synaptic pharmacology. J Neurophys 75: 1271-1282.

Wu SH, Ma CL, Kelly JB (2004) Contribution of AMPA, NMDA, and GABA receptors to temporal pattern of postsynaptic responses in the inferior colliculus of the rat. $J$ Neurosci $24: 4625-4634$.

Wu SH, Ma CM, Sivaramakrishnan S, Oliver DL (2002) Synaptic modification in neurons of the central nucleus of the inferior colliculus. Hear Res 168: 43-54.

Wu SH, Oertel D (1986) Inhibitory circuitry in the ventral cochlear nucleus is probably mediated by glycine. $J$ Neurosci 6: 2691-2706.

Wu SH, Oertel D (1987) Maturation of synapses and electrical properties of cells in the cochlear nuclei. Hear Res 30: 99-110.

Wyllie DJ, Behe P, Colquhoun D (1998) Single-channel activations and concentration jumps: comparison of recombinant NR1a/NR2A and NR1a/NR2D NMDA 
receptors. J Physiol (Lond) 510:1-18.

Wynne B, Harvey AR, Robertson D, Sirinathsinghi DJS (1995) Neurotransmitter and neuromodulator systems of the rat inferior colliculus and auditory brainstem studied by in situ hybridization. J Chem Neuroanat 9: 289-300.

Yang L, Pollak GD, Resler C (1992) GABAergic circuits sharpen tuning curves and modify response properties in the mustache bat inferior colliculus. $J$ Neurophysiol 68(5):1760-74.

Yang Y, Surette AM, Bishop D, Oliver DL (2000) Properties of local circuits in the central nucleus of the IC (CIC), SFN Abstr 26: 674.

Yasui Y, Kayahara T, Kuga Y, Katsuma N (1990) Direct projections from the globus pallidus to the inferior colliculus in the rat. Neurosci Lett 115: 121-125.

Zhang C, Granstrom L, Wong-Riley MT (1996) Deafferentation leads to a down-regulation of nitric oxide synthase in the rat visual system. Neurosci Lett 211: 61-64.

Zhang H, Kelly JB (2001) AMPA and NMDA receptors regulate responses of neurons in the rat's inferior colliculus. $J$ Neurophysiol 86: 871-880.

Zhang H, Kelly JB (2003) Glutamatergic and GABAergic regulation of neural responses in inferior colliculus to amplitude-modulated sounds. $J$ Neurophysiol 90(1):477-90.

Zhang H, Kelly JB (2006) Responses of neurons in the rat's dorsal cortex of the inferior colliculus to monaural and binaural acoustic stimulation. Proceedings of the 29th meeting of the Assoc Res Otolaryngol Baltimore, US., p. 47.

Zhang Y, Wu SH (2000) Long-term potentiation in the inferior colliculus studied in rat brain slice. Hear Res 147(1-2):92-103.

Zhang YF, Suga N, Yan J (1997) Corticofugal modulation of frequency processing in bat auditory system. Nature 387: 900-903.

Zhao M, Wu SH (2001) Morphology and physiology of neurons in the ventral nucleus of the lateral lemniscus in rat brain slices. J Comp Neurol 433: 255-271.

Zook JM, Casseday JH (1982) Origin of ascending projections to the inferior colliculus in the mustache bat, Pteronotus parnellii. J Comp Neurol 207: 14-28.

Zook JM, Casseday JH (1987) Convergence of ascending pathways at the inferior colliculus of the mustache bat, Pteronotus parnellii. J Comp Neurol 261(3): 347-61.

Zook JM, Winer JA, Pollak GD, Bodenhamer RD (1985) Topology of the central nucleus of the mustache bat's inferior colliculus: correlation of single unit properties and neuronal architecture. J Comp Neurol 231(4):530-46. 